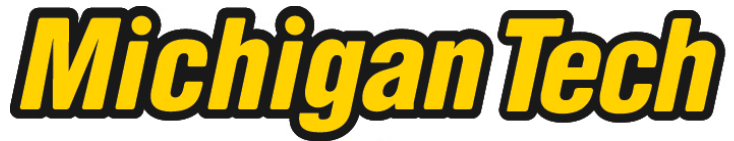 \\ Michigan Technological University Create the Future Digital Commons @ Michigan Tech
}

Dissertations, Master's Theses and Master's Reports - Open

Dissertations, Master's Theses and Master's

Reports

2011

Probe studies of a Hall thruster at low voltages

Jerry L. Ross

Michigan Technological University

Follow this and additional works at: https://digitalcommons.mtu.edu/etds

Part of the Mechanical Engineering Commons

Copyright 2011 Jerry L. Ross

\section{Recommended Citation}

Ross, Jerry L., "Probe studies of a Hall thruster at low voltages", Dissertation, Michigan Technological University, 2011.

https://doi.org/10.37099/mtu.dc.etds/405

Follow this and additional works at: https://digitalcommons.mtu.edu/etds

Part of the Mechanical Engineering Commons 


\title{
Probe Studies of a Hall Thruster at Low Voltages
}

\author{
by
}

Jerry L. Ross

\begin{abstract}
A Dissertation
Submitted in partial fulfillment of the requirements for the degree of Doctor of Philosophy in Mechanical Engineering - Engineering Mechanics
\end{abstract}

MICHIGAN TECHNOLOGICAL UNIVERSITY

2011 
Copyright (C) 2011 Jerry L. Ross 


\section{Michigan TeChNological University}

Department of Mechanical Engineering - Engineering Mechanics

This dissertation, entitled Probe Studies of a Hall Thruster at Low Voltages, by Jerry L. Ross is hereby approved in partial fulfillment of the requirements for the degree of Doctor of Philosophy in Mechanical Engineering - Engineering Mechanics.

Signatures:

Dissertation Advisor

Dr. Lyon B. King

Department Chair

Dr. William W. Predebon

Date 


\section{Contents}

1 Introduction 1

1.1 Thrust to Power Ratio . . . . . . . . . . . . . . . . . . . . 1

1.2 Scope . . . . . . . . . . . . . . . . . 4

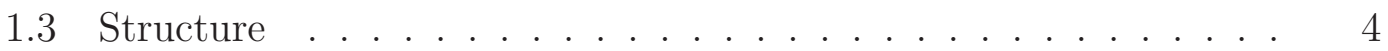

2 Background $\quad 7$

2.1 Brief History and Description of the Hall Thruster . . . . . . . 7

2.1.1 Hall Thruster Physics . . . . . . . . . . . . . . . . 8

2.2 Probes . . . . . . . . . . . . . . . . . . . . . . . 10

2.2.1 Retarding Potential Analyzer . . . . . . . . . . . . 10

2.2 .2 Faraday Probe . . . . . . . . . . . . . . . . . 12

2.2 .3 Langmuir Probe. . . . . . . . . . . . . . . . . . . . 12

2.3 Thrust Efficiency . . . . . . . . . . . . . . . . . . . . . . . . 14

2.3 .1 Voltage Utilization . . . . . . . . . . . . . . . 16

2.3.2 Current Efficiency . . . . . . . . . . . . . . . . . . 16

2.3.3 Velocity Distribution Function . . . . . . . . . . . . 16

2.3.4 Beam Divergence . . . . . . . . . . . . . . . . . 17

2.3.5 Thrust Efficiency Recombined . . . . . . . . . . . . . . 17

2.4 Low Voltage Hall thrusters . . . . . . . . . . . . . . . . . 17

2.5 Previous Research . . . . . . . . . . . . . . . . . . . . . . 19

2.6 Contribution . . . . . . . . . . . . . . . . . 20

3 Loss Mechanisms as a Function of Discharge Voltage 27

3.1 Equipment \& Facilities . . . . . . . . . . . . . . . . . . . . 27

3.1.1 BPT-2000 Hall Thruster . . . . . . . . . . . . . . 27

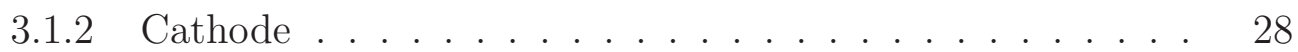

3.1.3 Vacuum Tank and Pumps . . . . . . . . . . . . . 28

3.1.4 Thrust Stand and Mass Flow Controllers . . . . . . . . . 28

3.2 Testing Matrices . . . . . . . . . . . . . . . . . 31

3.2.1 Thrust Stand Experiment . . . . . . . . . . . . . . 31

3.2 .2 Probe Study Experiment . . . . . . . . . . . . . . . . . 31 
3.3 Retarding Potential Probe . . . . . . . . . . . . . . . 31

3.4 Faraday Probe. . . . . . . . . . . . . . . . . . . . 33

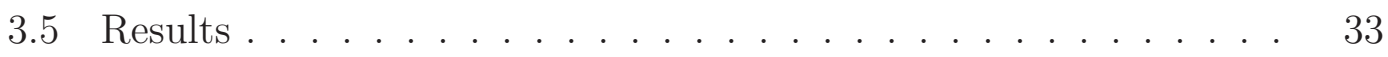

3.5.1 Thrust Efficiency . . . . . . . . . . . . . 33

3.5.2 Voltage Utilization Efficiency . . . . . . . . . . 36

3.5.3 Beam Divergence Efficiency . . . . . . . . . . . 36

3.5.4 Current Efficiency . . . . . . . . . . . . . . . . . . . . 38

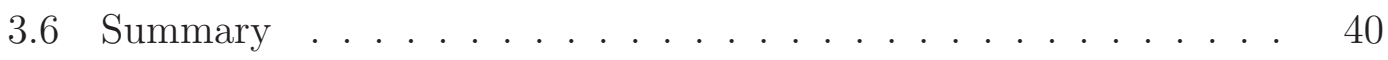

4 Loss Mechanisms as a Function of Magnet Current 45

4.1 Experimental Design . . . . . . . . . . . . . . . 46

4.1.1 Test Matrices . . . . . . . . . . . . . . . . 46

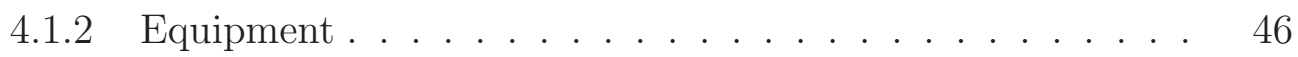

4.2 Thrust Stand Results . . . . . . . . . . . . . . . . . . . . . . . 48

4.2.1 Thrust Efficiency . . . . . . . . . . . . . . . . . . . . 48

4.2.2 Discharge Current . . . . . . . . . . . . . . . . . 48

4.2 .3 Thrust-to-Power . . . . . . . . . . . . . . 51

4.3 Probe Study . . . . . . . . . . . . . . . . . . . . . . . . . . . . . . . . . . . . . .

4.3.1 Current Efficiency . . . . . . . . . . . . . . . . . . 51

4.3.2 Voltage Utilization Efficiency .......... 53

5 Design of the High-Speed Probe System 59

5.1 Introduction . . . . . . . . . . . . . . . . . . . 59

5.2 Motion Table ..................... 61

5.3 Servo Motor . . . . . . . . . . . . . . . . . . . . 61

5.4 Fast Motion Camera Test . . . . . . . . . . . . . . . 63

5.5 Probe Construction . . . . . . . . . . . . . . . . 64

5.6 Analysis . . . . . . . . . . . . . . . . . 65

5.7 Data Aquisition . . . . . . . . . . . . . . . 66

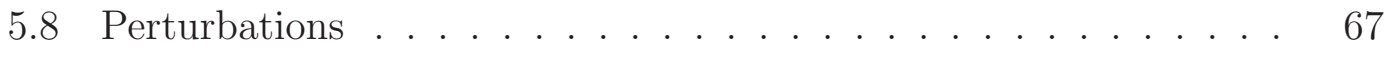

5.9 Summary . . . . . . . . . . . . . . . . . . 75

6 HPS Results $\quad 79$

6.1 Test Matrix . . . . . . . . . . . . . . . . . . . . . . . 79

6.2 Raw Results . . . . . . . . . . . . . . . . . . . . . . . . . . . . . . . . . . 79

6.3 Xenon Results . . . . . . . . . . . . . . . . . . 81

6.4 Krypton Results. . . . . . . . . . . . . . . . . . . 85

6.5 Ionization and Acceleration Zone locations . . . . . . . . . . . . . 87

6.6 Potential Drop in the Acceleration Zone . . . . . . . . . . . 89 
$\begin{array}{lll}7 & \text { Discussion } & 97\end{array}$

7.1 Efficiency vs Discharge Voltage . . . . . . . . . . . . . . . . . . 97

7.2 Efficiency vs Magnet Current . . . . . . . . . . . . . . . . . . . . 99

7.3 High-speed probe test . . . . . . . . . . . . . . . . 101

7.4 Separation Distance . . . . . . . . . . . . . . . . . . . . . . 109

7.5 Magnetic Field . . . . . . . . . . . . . . . . . . . . 110

7.6 Internal VS External . . . . . . . . . . . . . . . . . . 112

7.7 Conclusions .......................... 119

$\begin{array}{lr}\text { A HPS Data } & 123\end{array}$

A.1 Xenon . . . . . . . . . . . . . . . . . . . . . . 123

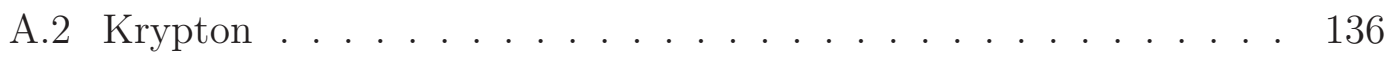




\section{Nomenclature}

$\Delta m \quad$ Change in spacecraft mass due to expended propellant

$\Delta t \quad$ trip time, $\mathrm{s}$

$\Delta V \quad$ velocity increment, $\mathrm{m} / \mathrm{s}^{2}$

$\dot{m} \quad$ flow rate of propellant as determined by the mass flow controller, $\mathrm{kg} / \mathrm{s}$

$\dot{m} \quad$ mass flow rate, $\mathrm{mg} / \mathrm{s}$

$\mathrm{V} \quad$ velocity vector, $\mathrm{m} / \mathrm{s}$

$A_{s} \quad$ surface area of the Langmuir probe, $\mathrm{m}^{2}$

$B \quad$ magnetic field strength, $\mathrm{G}$

e elementary charge, $\mathrm{C}$

$f_{0} \quad$ neutral mass fraction

$f_{1} \quad$ singly ionized mass fraction

$f_{2} \quad$ doubly ionized mass fraction

$f_{i} \quad$ ionization mass fraction of the propellant

$g$ gravitational constant, $\mathrm{m} / \mathrm{s}^{2}$

$I_{d} \quad$ discharge current, A

$I_{i} \quad$ exhausted ion current, A

$I_{\text {sat }}$ ion saturation current, A

$I_{s p} \quad$ specific impulse, $\mathrm{s}$ 
$j(\theta)$ ion current density, $\mathrm{A} / \mathrm{m}^{2}$

$k \quad$ Boltzmann constant, $\mathrm{m}^{2} \mathrm{~kg} \mathrm{~s}^{-2} \mathrm{~K}^{-1}$

$M \quad$ spacecraft mass, $\mathrm{kg}$

$m$ mass of a xenon atom/ion, $\mathrm{kg}$

$m_{e} \quad$ electron mass, $\mathrm{kg}$

$m_{i} \quad$ ion mass, $\mathrm{kg}$

$m_{p} \quad$ mass of the power supply, $\mathrm{kg}$

$n_{i} \quad$ ion density, $\mathrm{m}^{-3}$

$P \quad$ kinetic power delivered to the spacecraft, $\mathrm{W}$

$p \quad$ proportionality constant

$P_{k} \quad$ directed kinetic power making up the thrust component, W

$P_{s} \quad$ supply output power $I_{d} V_{d}, \mathrm{~W}$

$Q \quad$ average charge state of the ionized propellant

$q \quad$ charge number

$r_{e} \quad$ electron Larmor radius

$r_{L} \quad$ Larmor radius

$T$ thrust, $\mathrm{N}$

$T_{e} \quad$ electron temperature, $\mathrm{J}$

$T_{e V} \quad$ electron temperature, $\mathrm{eV}$

$U_{e} \quad$ ion velocity, $\mathrm{m} / \mathrm{s}^{2}$

$v_{\perp} \quad$ perpendicular velocity w.r.t. the magnetic field

$V_{d} \quad$ discharge voltage (anode potential), $\mathrm{V}$

$V_{f} \quad$ floating potential, eV

$V_{p} \quad$ plasma potential, eV 


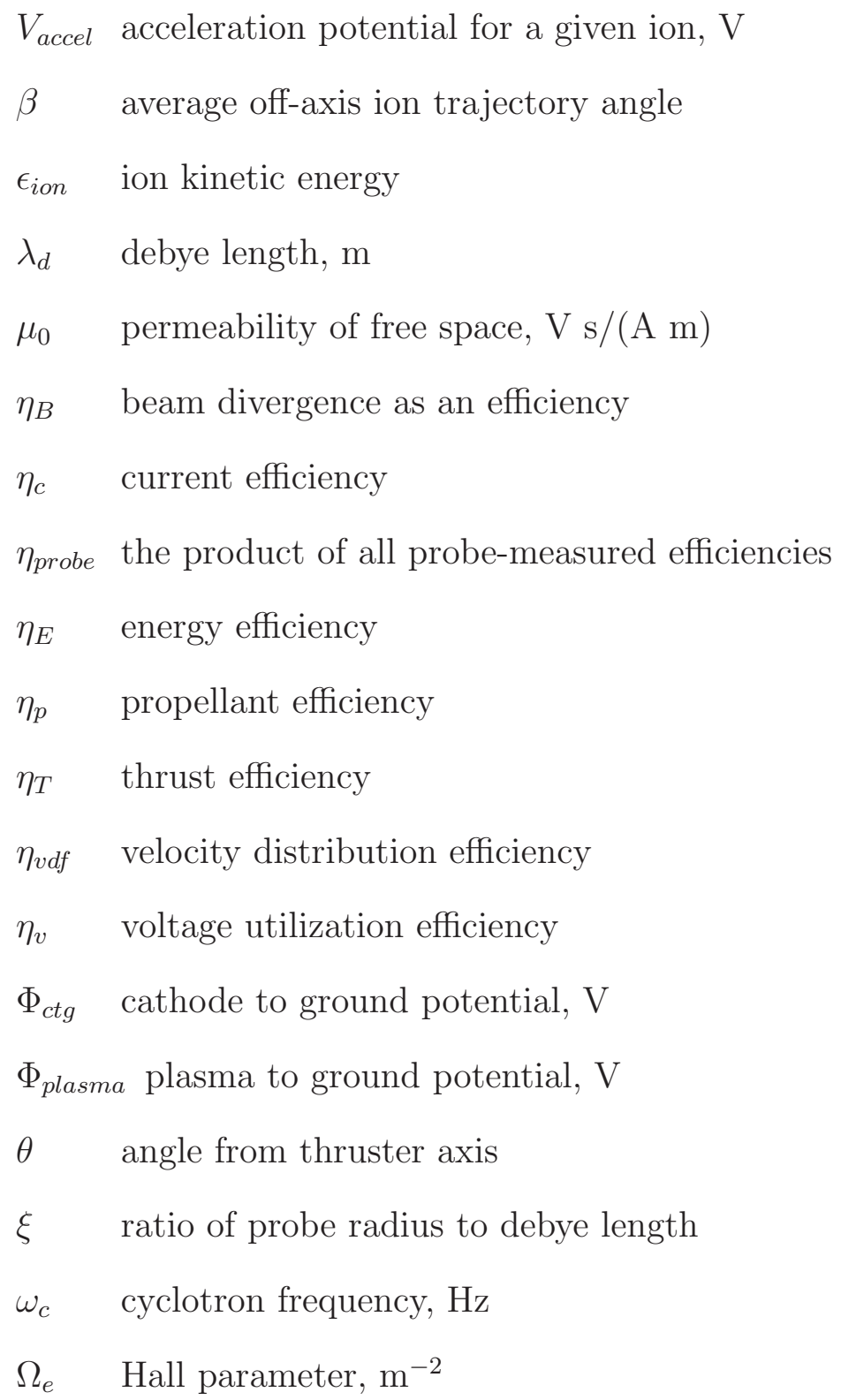




\section{List of Figures}

2.1 Cartoon cross-section of Hall thruster and cathode system . . . .

2.2 One quarter cross section of a Hall thruster. The yellow lines represent the radial magnetic field and the red line represents a typical plasma potential profile in the Hall thruster. . . . . . . . . . . .

2.3 Internal diagram of the RPA probe where E.R. stands for electron repellor. . . . . . . . . . . . . . . . . . . . . 11

2.4 Potential structure created by the grids within the RPA . . . . . . 11

2.5 Ideal single Langmuir trace . . . . . . . . . . . . . . . . . . 13

2.6 Performance characteristics of modern Hall thrusters in research and in industry. Larson, C. W., Brown, D. L., and Hargus, W. A., Thrust Efficiency, Energy Efficiency, and the Role of the VDF in Hall Thruster Performance Analysis, 43rd AIAA/ASME/SAE/ASEE Joint Propulsion Conference and Exhibit, Vol. AIAA 2007-5270, Cincinnati, Ohio, July 8-11, 2007 . . . . . . . . . 18

3.1 The Aerojet BPT-2000 Hall thruster . . . . . . . . . . . . . . 28

3.2 A top down view of the inside of the xenon Hall thruster testing facility . . . . . . . . . . . . . . . . . . 29

3.3 RPA probe on the left and Faraday Probe on the right . . . . . . 32

3.4 Faraday probe sweeps $250 \mathrm{~mm}$ downstream for $3 \mathrm{mg} / \mathrm{s}$ flow rate at five different discharge voltages . . . . . . . . . . . . . .

3.5 Thrust efficiency as determined by thrust measurements. Error bars are calculated based on the reproducibility of calibration measurements both during operation and when the thruster is turned off. . . . . . . . . . . . . . . . . . . .

3.6 Discharge current at $\max \eta_{T}$. Uncertainty is $\pm 0.05 \mathrm{~A}$ based on manufacturer's specified uncertainty for the discharge power supply. 35

3.7 Thrust to Power ratio for the BPT-2000 operating on $3 \mathrm{mg} / \mathrm{s}$ of xenon. . . . . . . . . . . . . . . . 36

$3.8 \bar{\epsilon} / e V_{d}$ as calculated from RPA data $0^{\circ}$ off axis and $550 \mathrm{~mm}$ downstream . . . . . . . . . . . . . . . . . . . 37 
$3.9 \bar{\epsilon} / e V_{d}$ as calculated from RPA data $15^{\circ}$ off axis and $550 \mathrm{~mm}$ downstream . . . . . . . . . . . . . . . . 37

$3.10 \bar{\epsilon} / e V_{d}$ as calculated from RPA data $30^{\circ}$ off axis and $550 \mathrm{~mm}$ down-

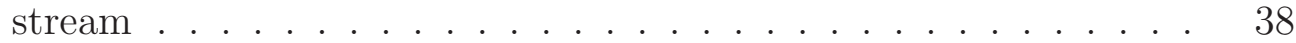

3.11 Beam divergence efficiency as calculated by Eq. 3.3 from Faraday probe sweeps $250 \mathrm{~mm}$ downstream . . . . . . . . . . . 39

3.12 Current efficiency as a function of discharge voltage . . . . . . . . 39

4.1 Thrust efficiency for xenon . . . . . . . . . . . . . . . . . . . . . . 49

4.2 Thrust efficiency for krypton . . . . . . . . . . . . . . . . . . . . . 49

4.3 Glow Discharge . . . . . . . . . . . . . . . . . . . 49

4.4 Collimated Beam . . . . . . . . . . . . . . . . . . 49

4.5 Discharge current for xenon . . . . . . . . . . . . . . 50

4.6 Discharge current for krypton . . . . . . . . . . . . . 50

4.7 Thrust to power results for xenon in $\mathrm{mN} / \mathrm{kW}$. . . . . . . . . 52

4.8 Thrust to power results for krypton in $\mathrm{mN} / \mathrm{kW}$. . . . . . . . . 52

4.9 Current efficiency for krypton . . . . . . . . . . . . . . . 52

4.10 Voltage utilization for xenon . . . . . . . . . . . 53

4.11 Voltage utilization for krypton . . . . . . . . . . . . . 53

4.12 Raw RPA IV curves for krypton at $125 \mathrm{~V}$ for various magnet current values. . . . . . . . . . . . . . . . . 54

4.13 Normalized version of figure 4.12 showing RPA IV curves for krypton at $125 \mathrm{~V}$ for various magnet current values. . . . . . . . . . .

4.14 Normalized -dI/dV RPA traces for krypton at $125 \mathrm{~V}$ for various magnet current values. . . . . . . . . . . . . .

5.1 Resident melting time of an electrostatic probe in the discharge chamber of a Hall thruster as a function of magnetic and electric field strength: J. M. Haas and A. D. Gallimore. "Development of a high-speed, reciprocating electrostatic probe system for hall thruster interrogation". Review of Scientic Instruments, 71(11), November 2000. . . . . . . . . . . . . . . . .

5.2 Assembled high-speed motion table with mounted double Langmuir probe............................ 62

5.3 Servo motor to motion table coupler . . . . . . . . . . . 63 
5.4 Fast motion camera results showing the probe completing the simulated $\pm 18 \mathrm{~mm}$ round trip from the exit plane to the anode and back in less than $60 \mathrm{~ms}$. The motion table is driving the platform from the right to the left in the pictures. The middle picture shows the point of furthest travel where the center screw in the platform is visible. The first last pictures, nearly identical, show the platform entering the frame and leaving the frame respectively. The distance travelled between frames (a) and (b) is $18 \mathrm{~mm}$ and the same between frames (b) and (c). . . . . . . . . . .

5.5 Close-up of tungsten probe tips for the double Langmuir probe . .

5.6 Voltage and capacitive current of the sweep circuit. Current is in black and voltage in grey. . . . . . . . . . . . . . . . . . . .

5.7 Double probe circuit diagram ................

5.8 Discharge current measurements for xenon at $125 \mathrm{~V}$ as the Langmuir probe is inserted into the discharge chamber. The colored horizontal dashed lines represent the maximum and minimum current where the center bold line represents the average current. The vertical dotted lines represent the exit plane as its passed on the way into the discharge chamber and on the way back out. . . . . .

5.9 Discharge current measurements for xenon at $200 \mathrm{~V}$ as the Langmuir probe is inserted into the discharge chamber. The colored horizontal dashed lines represent the maximum and minimum current where the center bold line represents the average current. The vertical dotted lines represent the exit plane as its passed on the way into the discharge chamber and on the way back out. . . . . .

5.10 Discharge current measurements for xenon at $300 \mathrm{~V}$ as the Langmuir probe is inserted into the discharge chamber. The colored horizontal dashed lines represent the maximum and minimum current where the center bold line represents the average current. The vertical dotted lines represent the exit plane as its passed on the way into the discharge chamber and on the way back out. . . . . .

5.11 Discharge current measurements for krypton at $125 \mathrm{~V}$ as the Langmuir probe is inserted into the discharge chamber. The colored horizontal dashed lines represent the maximum and minimum current where the center bold line represents the average current. The vertical dotted lines represent the exit plane as its passed on the way into the discharge chamber and on the way back out. . . . . 
5.12 Discharge current measurements for krypton at $200 \mathrm{~V}$ as the Langmuir probe is inserted into the discharge chamber. The colored horizontal dashed lines represent the maximum and minimum current where the center bold line represents the average current. The vertical dotted lines represent the exit plane as its passed on the way into the discharge chamber and on the way back out. . . . . .

6.1 Raw current data as a function of axial position as recorded by the HPS. This is the entire recorded data set, however, only the portions in blue are used for data analysis. The blue data, and all red data preceding it represents the inward bound travel of the probe. $-20 \mathrm{~mm}$ (relative to the exit plane) is the end of travel where the probe stops just in front of the anode and reverses motion. All data to the right of the blue section represents the data during the return trip of the probe. . . . . . . . . . . . . . .

6.2 Typical example of raw current and voltage sweep data for a single extracted double probe trace. The motion table moves $1 \mathrm{~mm}$ during the signal acquisition. Data is shown before any manipulation or removal of the influence of line capacitance. . . . . . . . . . . . . 82

6.3 High-speed probe data for xenon . . . . . . . . . . . . . . . . . 82

6.4 High-speed probe data for xenon . . . . . . . . . . . . . . . 83

6.5 High-speed probe data for xenon . . . . . . . . . . . . . . . 83

6.6 High-speed probe data for xenon . . . . . . . . . . . . . 84

6.7 High-speed probe data for krypton . . . . . . . . . . . . . . . . 85

6.8 High-speed probe data for krypton . . . . . . . . . . . . . . . 85

6.9 High-speed probe data for krypton . . . . . . . . . . . . . . . . 86

6.10 High-speed probe data for krypton . . . . . . . . . . . . . . 86

6.11 Potential drop across the acceleration zone for xenon . . . . . . . 90

6.12 Potential drop across the acceleration zone for krypton . . . . . . 91

6.13 An example of floating potential, plasma potential and electron energy for krypton at $300 \mathrm{~V}$. . . . . . . . . . . . . 92

6.14 An example of floating potential, plasma potential and electron energy for krypton at $150 \mathrm{~V}$. . . . . . . . . . . . . 92

$7.1 \eta$ values for $3 \mathrm{mg} / \mathrm{s}$ of xenon at maximum thrust efficiency . . . . 98

$7.2 \eta$ values for $4 \mathrm{mg} / \mathrm{s}$ of xenon at maximum thrust efficiency . . . . 98

$7.3 \eta$ values for $5 \mathrm{mg} / \mathrm{s}$ of xenon at maximum thrust efficiency . . . . 99

7.4 Magnetic $p$ value for xenon . . . . . . . . . . . . . . . . . . 102

7.5 Magnetic $p$ value for krypton . . . . . . . . . . . . . . . . . . . 102

$7.6 \xi$ Values for high/low voltages and both propellants . . . . . . . . 102

7.7 Krypton ion density . . . . . . . . . . . . . . . . . . . . 104 
7.8 Xenon ion density . . . . . . . . . . . . . . . . . . . 104

7.9 High-speed probe results for xenon at $125 \mathrm{~V} 0.50 \mathrm{~A}$. . . . . . . . 105

7.10 An example of the uncertainty in the ionization zone location as shown between the black arrows . . . . . . . . . . . . . 106

7.11 Acceleration zone location as a function of magnet current for krypton 107

7.12 Ionization zone location as a function of magnet current for krypton 107

7.13 Acceleration zone location as a function of magnet current for xenon 107

7.14 Ionization zone location as a function of magnet current for xenon 107

7.15 The separation distance between the acceleration zone and the ionization zone for krypton. Negative values indicate an ionization zone location upstream of the acceleration zone. . . . . . . . . 109

7.16 The separation distance between the acceleration zone and the ionization zone for xenon. Negative values indicate an ionization zone location upstream of the acceleration zone. . . . . . . . . . . 110

7.17 Axial magnetic field profile for the BPT-2000 along centerline . . 111

7.18 Magnetic field strength at the peak ion density location for xenon 113

7.19 Magnetic field strength at the acceleration zone location for xenon 113

7.20 Magnetic field strength at the peak ion density location for krypton 113

7.21 Magnetic field strength at the acceleration zone location for krypton 113

7.22 The green box represents the region over which the internal voltage utilization efficiency was calculated . . . . . . . . . . . 114

7.23 Internal voltage utilization efficiency as calculated from the HPS dataset compared to the voltage utilization efficiency calculated

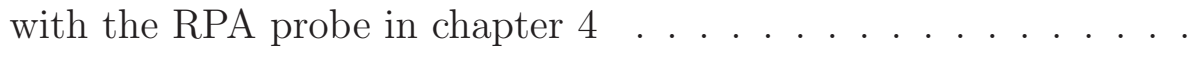

7.24 Internal voltage utilization efficiency as calculated from the HPS dataset compared to the voltage utilization efficiency calculated with the RPA probe in chapter 4 . . . . . . . . . . . . .

7.25 Internal voltage utilization efficiency as calculated from the HPS dataset compared to the voltage utilization efficiency calculated with the RPA probe in chapter 4 . . . . . . . . . . . . 116

7.26 Internal voltage utilization efficiency as calculated from the HPS dataset compared to the voltage utilization efficiency calculated with the RPA probe in chapter 4 . . . . . . . . . . . . . . . 117

7.27 Internal voltage utilization efficiency as calculated from the HPS dataset compared to the voltage utilization efficiency calculated with the RPA probe in chapter 4 . . . . . . . . . . . . .

7.28 Internal voltage utilization efficiency as calculated from the HPS dataset compared to the voltage utilization efficiency calculated with the RPA probe in chapter 4 . . . . . . . . . . . 118 
xviii

7.29 Internal voltage utilization efficiency as calculated from the HPS dataset compared to the voltage utilization efficiency calculated with the RPA probe in chapter 4 . . . . . . . . . . . . . . . 118

A.1 High-speed probe results for Xenon . . . . . . . . . . . . . . . . . 123

A.2 High-speed probe results for Xenon . . . . . . . . . . . . . . . . 124

A.3 High-speed probe results for Xenon . . . . . . . . . . . . . . . . 124

A.4 High-speed probe results for Xenon . . . . . . . . . . . . . . . . 125

A.5 High-speed probe results for Xenon . . . . . . . . . . . . . . . . 125

A.6 High-speed probe results for Xenon . . . . . . . . . . . . . . 126

A.7 High-speed probe results for Xenon . . . . . . . . . . . . . . 126

A.8 High-speed probe results for Xenon . . . . . . . . . . . . . . . . . 127

A.9 High-speed probe results for Xenon . . . . . . . . . . . . . . . 127

A.10 High-speed probe results for Xenon . . . . . . . . . . . . . . . 128

A.11 High-speed probe results for Xenon . . . . . . . . . . . . . . . . . 128

A.12 High-speed probe results for Xenon . . . . . . . . . . . . . . 129

A.13 High-speed probe results for Xenon . . . . . . . . . . . . . . . . . 129

A.14 High-speed probe results for Xenon . . . . . . . . . . . . . . 130

A.15 High-speed probe results for Xenon . . . . . . . . . . . . . . . . . 130

A.16 High-speed probe results for Xenon . . . . . . . . . . . . . . 131

A.17 High-speed probe results for Xenon . . . . . . . . . . . . . . . . . 131

A.18 High-speed probe results for Xenon . . . . . . . . . . . . . . . . . 132

A.19 High-speed probe results for Xenon . . . . . . . . . . . . . . . . . 132

A.20 High-speed probe results for Xenon . . . . . . . . . . . . . . . 133

A.21 High-speed probe results for Xenon . . . . . . . . . . . . . . 133

A.22 High-speed probe results for Xenon . . . . . . . . . . . . . . . . . 134

A.23 High-speed probe results for Xenon . . . . . . . . . . . . . . . . 134

A.24 High-speed probe results for Xenon . . . . . . . . . . . . . . 135

A.25 High-speed probe results for Krypton . . . . . . . . . . . . . . . . 136

A.26 High-speed probe results for Krypton . . . . . . . . . . . . . . . 136

A.27 High-speed probe results for Krypton . . . . . . . . . . . . . . . 137

A.28 High-speed probe results for Krypton . . . . . . . . . . . . . . . . 137

A.29 High-speed probe results for Krypton . . . . . . . . . . . . . . . . 138

A.30 High-speed probe results for Krypton . . . . . . . . . . . . . . . . 138

A.31 High-speed probe results for Krypton . . . . . . . . . . . . . . . . 139

A.32 High-speed probe results for Krypton . . . . . . . . . . . . . . . . 139

A.33 High-speed probe results for Krypton . . . . . . . . . . . . . . . . 140

A.34 High-speed probe results for Krypton . . . . . . . . . . . . . . . . 140

A.35 High-speed probe results for Krypton . . . . . . . . . . . . . . . . 141

A.36 High-speed probe results for Krypton . . . . . . . . . . . . . . . . 141

A.37 High-speed probe results for Krypton . . . . . . . . . . . . . . . . 142 
A.38 High-speed probe results for Krypton . . . . . . . . . . . . . . . . 142

A.39 High-speed probe results for Krypton . . . . . . . . . . . . . . . . 143

A.40 High-speed probe results for Krypton . . . . . . . . . . . . . . . . 143

A.41 High-speed probe results for Krypton . . . . . . . . . . . . . . . . 144

A.42 High-speed probe results for Krypton . . . . . . . . . . . . . . . . 144

A.43 High-speed probe results for Krypton . . . . . . . . . . . . . . . . 145

A.44 High-speed probe results for Krypton . . . . . . . . . . . . . . . . 145

A.45 High-speed probe results for Krypton . . . . . . . . . . . . . . . . 146

A.46 High-speed probe results for Krypton . . . . . . . . . . . . . . . . 146

A.47 High-speed probe results for Krypton . . . . . . . . . . . . . . . . 147

A.48 High-speed probe results for Krypton . . . . . . . . . . . . . . . . 147

A.49 High-speed probe results for Krypton . . . . . . . . . . . . . . . . 148 


\section{List of Tables}

3.1 Mass flow system . . . . . . . . . . . . . . . .

3.2 Thrust stand testing matrix. Many of the lower voltages were not recorded for 4 and $5 \mathrm{mg} / \mathrm{s}$. . . . . . . . . . . . . . .

3.3 Probe study testing matrix. The probe study was conducted over a subset of the thrust stand testing matrix. . . . . . . . . . . . 31

3.4 Comprehensive summary of all $\eta$ values . . . . . . . . . . . . . . 41

4.1 Test parameters for xenon and krypton . . . . . . . . . . . . . . . 47

4.2 Magnet current values corresponding to maximum efficiency and minimum current to the anode for krypton . . . . . . . . . 50

5.1 SureServo SVL-200 Servo Motor Specifications . . . . . . . . . . 61

6.1 Test parameters for xenon and krypton . . . . . . . . . . . . . 80

6.2 Krypton Ionization Zone. Values are given in $\mathrm{mm}$ from the exit plane. Negative values indicate locations internal to the discharge chamber. . . . . . . . . . . . . . . . . 88

6.3 Xenon Ionization Zone. Values are given in $\mathrm{mm}$ from the exit plane. Negative values indicate locations internal to the discharge chamber. 88

6.4 Krypton Acceleration region. Values are given in $\mathrm{mm}$ from the exit plane. Negative values indicate locations internal to the discharge chamber. . . . . . . . . . . . . . . . . .

6.5 Xenon Acceleration Region. Values are given in $\mathrm{mm}$ from the exit plane. Negative values indicate locations internal to the discharge chamber. . . . . . . . . . . . . . . . .

7.1 The upstream uncertainty in the ionization zone location as measured from the peak ion density toward the anode . . . . . . . 106

7.2 The separation distance between the acceleration zone and the ionization zone for krypton. Negative values indicate an ionization zone location upstream of the acceleration zone. . . . . . . . . 
7.3 The separation distance between the acceleration zone and the ionization zone for xenon. Negative values indicate an ionization zone location upstream of the acceleration zone. . . . . . . . . . . 111 


\section{Chapter 1}

\section{Introduction}

Electric propulsion (EP) devices, such as the Hall thruster, are gaining use for space travel against their chemically propelled counterparts because of the propellant mass savings that EP can offer. This reduction in propellant mass allows for a lighter spacecraft and/or a more massive payload. Many missions, however, are time sensitive and electric propulsion devices are not capable of producing the levels of thrust necessary to meet the trip time demands. In these missions, chemical thrusters must be used as the primary propulsion device at the expense of a increased onboard propellent mass. The result is that many of today's spacecraft have both a primary propulsion device such as a chemical rocket as well as an auxiliary device for stationkeeping tasks (often an electric propulsion rocket such as a Hall thruster). Dual systems require dual propellant tanks, power supplies, mass flow controllers etc. Therefore, there is a desire in the electric propulsion community to create an EP device that can function both as a primary and auxiliary device for a spacecraft. For a Hall thruster to be capable of this, it would have to exhibit efficient throttle ability in thrust to function as a main thruster in addition to its present capabilities as an auxiliary thruster for orbit correction. By doing so, the propulsion system could be streamlined onboard spacecraft, reducing complexity (increased reliability) and reduction of the total system mass.

\subsection{Thrust to Power Ratio}

In space flight, the amount of time it takes to complete a maneuver is called the trip time and is related to the required velocity change, spacecraft mass, and thrust by

$$
\Delta t=\frac{M \Delta V}{T}
$$


The velocity change, $\Delta V$, is a fixed mission parameter for a given mission and is independent of the spacecraft in use, including propulsion system, mass, etc. ${ }^{1}$.

As previously mentioned, the thrust output for a typical electric propulsion rocket is much smaller than that of a chemical rocket, and is not trivial to increase. The difficulty in increasing the thrust stems from the mathematical relationship between thrust and power. The calculation of thrust is given by

$$
\text { Thrust }=\dot{m} U_{e}
$$

where $\dot{m}$ is the mass flow rate of propellant and $U_{e}$ represents the exit velocity of the ionized propellant. This consumes

$$
\text { Power }=\frac{1}{2} \dot{m} U_{e}^{2}
$$

total kinetic power. Now, by recasting the thrust as function of power, we get

$$
\text { Thrust }=\frac{2 P_{k}}{U_{e}}
$$

where $P_{k}$ is the directed kinetic power in the exhausted ion beam. The term 'directed' is included to account for the beam divergence which is not a power loss mechanism but is accounted for in the thrust efficiency. The beam divergence is explained in detail in chapter 2.

The level of kinetic power in the ion beam depends on how efficiently the electrical power is being converted to thrust

$$
P_{k}=\eta_{T} P_{s}
$$

where $P_{s}$ is the input electrical power. Electrical thrusters of all varieties are often characterized by their thrust efficiency $\left(\eta_{T}\right)$ values.

Ostensibly, increasing the thrust becomes a function of increasing the power supplied to the thruster according to equation 1.4. Unlike in a chemical thruster, where this power comes from the energy stored in the chemical bonds of the propellant, an electrical thruster gets its power from an onboard power plant (PPU) that converts solar or nuclear energy into electricity. Available onboard power from PPUs are limited at present to tens of $\mathrm{kW}$ which limits the thrust of EP devices to at present to hundreds of $\mathrm{mN}$. Furthermore, the mass of the PPU scales with its power output,

$$
P_{s}=\propto m_{p}=\frac{2 \eta_{T} T}{U_{e}}
$$

\footnotetext{
${ }^{1}$ For example LEO-GEO requires a $\Delta V$ of $4 \mathrm{~km} / \mathrm{s}$.
} 
By increasing the onboard power supply, the total expended propellent mass needed for the mission, $\Delta m$, is decreased, while the $m_{p}$ is increased. Thus, missions are designed to minimize $m_{p}+\Delta m$ to maximize the deliverable payload $[1]$.

Without the ability to increase the onboard power, focus must move toward trying to increase the thrust-to-power ratio,

$$
\frac{T}{P_{s}}=\frac{2 \eta_{T}}{U_{e}}
$$

which means either decreasing the exit velocity or increasing the thrust efficiency.

In the past decade, there has been great progress in increasing thrust efficiencies from benchmark levels of $50 \%$ on the Russian SPT-100 to upwards of $70 \%$ in some laboratory Hall thrusters [2, 3]. For many missions where EP could be considered, however, a several fold increase in thrust is necessary where even $100 \%$ thrust efficiency will not increase the thrust-to-power ratio to necessary levels.

Alternatively, to decrease the propellant exit velocity, the acceleration voltage,

$$
\frac{1}{2} m U_{e}^{2}=q V_{\text {accel }}
$$

, must be decreased, where $V_{\text {accel }}$ is approximately the voltage applied to the anode

$$
V_{\text {accel }} \approx V_{d}
$$

Decreasing the voltage applied to the anode (discharge voltage) on modern Hall thrusters causes decreases in thrust efficiency, $\eta_{T}$. Experimental data has shown that the decrease is so severe, that the thrust-to-power ratio declines instead of increasing. Thus, the problem of a high thrust-to-power ratio reverts back to increasing the thrust efficiency at low discharge voltages.

There is a large gap in the empirical data available for low voltage operation of Hall thrusters. As a fledging technology, it was discovered that Hall thrusters did not perform well at voltages below $\sim 300 \mathrm{~V}$. Therefore, experiments were focused on the areas where the thruster performed the best, namely, high voltages. The interest in low voltage or multi-mode operation of a Hall thruster has only seen a real push in the last 5-6 years. Furthermore, although some of the loss mechanisms have been flushed out, not all of physics behind these loss mechanisms are understood. To further complicate matters, some of the data available exhibit non-monotonic behavior that is not conventionally explained by the models used in the field.

It is therefore the aim of this work to examine plasma processes inside the discharge chamber of a Hall thruster at low voltages, and to compare those data to internal data for high voltages and external probe data for both high and 
low voltages. Internal probe data offers a deeper look into the operation of a Hall thruster that external probe data and thrust stand measurements cannot capture. To broaden the results of these experiments, many of the external probe studies and all of the internal work will be conducted using both xenon and krypton as propellant sources.

\section{$1.2 \quad$ Scope}

The work herein is an experimental study, and the parameters explored are those that can be controlled without altering the thruster itself (i.e. power supplies, mass flow controllers etc). The structure of the Hall thruster, discharge chamber dimensions, magnetic circuit, and cathode were not altered at any point during operation. This study was conducted on one production grade Hall thruster under the assumption that many of the phenomena discovered here are applicable to other Hall thrusters exhibiting similar inefficiencies. Identical testing on several other Hall thrusters would serve to refute or support whether or not the data can be considered universally applicable. Many of the results are intended to be used to optimize future Hall thruster models, however, the scope of this body of work is to highlight a comparison to current model data in the field.

\subsection{Structure}

The next chapter of the thesis details all of the necessary background information including probe theory, Hall thruster operation, and previous research. Chapter 2 also catalogs the probe techniques in the external and internal studies. Chapter 3 , 'Loss mechanisms as a function of discharge voltage,' begins with a discussion of apparatus including the thrust stand and vacuum chamber used in testing. Chapter 4 outlines the second test, 'Loss mechanisms as a function of magnet current,' and the results therein. Much of the detailed discussion is saved for chapter 7 . Chapter 5 details the construction of the high-speed probe system used to perform the internal probe tests, and the results appear subsequently in chapter 6. Lastly, chapter 7 is reserved for discussion, concluding remarks and note on future work. 


\section{Bibliography}

[1] Jahn, R. G., Physics of Electric Propulsion, Dover Publications, 1968.

[2] Goebel, D. and Katz, I., Fundamentals of Electric Propulsion, John Wiley and Sons, Inc., 2008.

[3] Brown, D. L., Reid, B. M., and Gallimore, A. D., "Performance Characterization and Design Verification of a 6-kW Laboratory Model Hall Thruster," 54th JANNAF Propulsion Meeting, Denver, Colorado, May 14-17 2007. 
CHAPTER 1 


\section{Chapter 2}

\section{Background}

This chapter begins with a rudimentary explanation of Hall thruster operation followed by a mathematical derivation and description of efficiency terms found throughout this dissertation. Next, is a section on probe theory and diagnostic tools used. Then, a literature review of previous work done in the low voltage and/or high thrust-to-power areas of Hall thruster research is presented.

\subsection{Brief History and Description of the Hall Thruster}

The fundamentals for electric propulsion were put to paper by Robert H. Goddard at the turn of the twentieth century, however the Hall thruster didn't come into existence until the 1960's [1]. At that time, the focus of research for closed-drift thrusters (what are known as Hall thrusters today) was in the 5000-10,000 second $I_{s p}$ range. Specific impulse, $I_{s p}$, refers to the exit velocity of the plume divided by gravity,

$$
I_{s p}=\frac{U_{e}}{g} .
$$

Due to electron back streaming that rose above classical diffusion models [2], it was determined that efficient operation was not possible in comparison to the ion thruster. Research on the Hall-thruster was abandoned in the US in the 1970's, however, it continued in the Soviet Union.

At the conclusion of the Cold War, Russia surfaced with 20 years of technology in the SPT-100 Hall thruster and many other devices displaying viable technology for near-earth missions in the range of 1500-2000 s $I_{s p}$. Since then, Hall thruster research was rebooted at the NASA Glenn research center in Ohio in the early 
1990's and has spread to a couple dozen universities, government agencies, and private businesses across the country.

\subsubsection{Hall Thruster Physics}

The Hall thruster can broken down into its three main components: the anode, cathode, and magnetic circuit.

The anode is a multifaceted device that is responsible for maintaining the electric field, functioning as the inlet for neutral propellant and being the catch-all for upstreaming electrons. The device is typically porous to function as the neutral feed line and made from a conductor, often stainless steel, that is electrically isolated from the rest of the Hall thruster and held at a high potential that is called the anode/discharge potential/voltage. Barring loss mechanisms, it is the anode potential that determines the velocity at which the propellant is accelerated. The anode must be constructed such that it is robust enough to withstand joule heating from the back streaming electrons. Stainless steel is sufficient when working with xenon and krypton, but condensible propellants that include active heating elements often require an anode forged from a refractory metal such as molybdenum [3]. A magnetic field is employed near the exit plane in a Hall

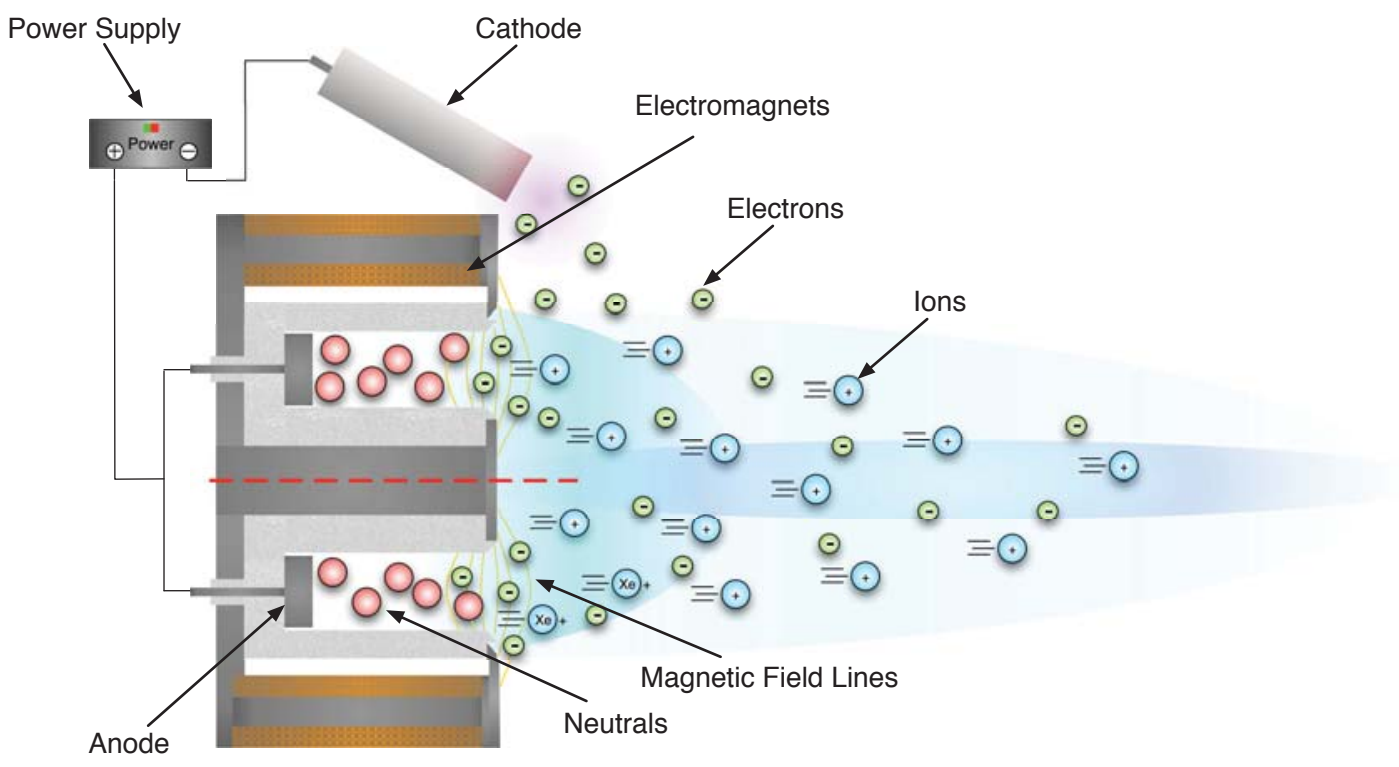

Figure 2.1: Cartoon cross-section of Hall thruster and cathode system

thruster to impede the motion of the electrons. Electromagnets are placed around the perimeter and in the center of the device to create a radial field across the 
exit plane, perpendicular to the electric field created between the anode-cathode. The strength of the magnetic field is tuned, via the magnet coil current, in order to keep the electron Larmor radius small and the ion Larmor radius large when compared to the dimensions of the discharge chamber. The general equation for the Larmor radius is

$$
r_{L}=\frac{v_{\perp}}{\omega_{c}}
$$

where $v_{\perp}$ is the perpendicular velocity and $\omega_{c}$ is the cyclotron frequency defined as

$$
\omega_{c}=\frac{|q| B}{m} .
$$

In the absence of the magnetic field in a Hall thruster, the electron motion would be axial, and therefore the thermal velocity of the electrons can be substituted for the perpendicular velocity in equation 2.2 to get

$$
r_{e}=\frac{v_{t h}}{\omega_{c}}=\frac{m}{e B} \sqrt{\frac{8 k T_{e}}{\pi m}}=\frac{1}{B} \sqrt{\frac{8 m}{\pi e} T_{e V}} \ll L
$$

where $T_{e V}$ is the electron temperature in $\mathrm{eV}$, and $L$ is taken as the characteristic length or plasma length inside the discharge chamber. L can be taken as the length of the discharge chamber for an order of magnitude comparison. Typical magnetic field values are $\sim 200 \mathrm{G}$ which gives an electron Larmor radius of $\sim 1 \mathrm{~mm}$ and an ion Larmor radius of $\sim 150 \mathrm{~cm}$. In practice, this means that the magnetic field confines the electrons whereas the ions are largely unaffected by it due to their larger mass. The Hall parameter,

$$
\Omega_{e}^{2}=\frac{\omega_{c}^{2}}{v^{2}}
$$

, is a gauge of this, and the electrons are said to be magnetized when $\Omega_{e}^{2} \gg 1$. The goal of magnetizing the electrons is to reduce their cross field mobility, trapping them at the the exit plane. The electrons are trapped gyrating along magnetic field lines and this way create equipotential lines. The collection of equipotential lines creates a local gradient in plasma potential violating plasma neutrality and creating a potential hill for the ions to fall through. The peak magnetic field is typically the point of greatest potential gradient and makes up what is called the acceleration zone.

Ion production is the result of electron-neutral collisions near the exit plane. Pressure gradients cause diffusion of the neutral propellant downstream toward the exit plane where the highly energetic electrons are trapped on field lines. During electron-neutral collisions, if the electrons transfer energy in excess of the ionization potential, the neutral splits into an ion electron pair. The electron-neutral 
cross sections are a function of species, density, and electron temperature. Thus, propellants are chosen based on the desire for high mass, high electron-neutral cross section coefficient, and low ionization potential. The newly formed positively charged ions are repelled by the anode and are ejected out of the thruster, while the electrons work their way towards the anode to eventually be collected and pumped through the power supply to the cathode. The cathode, perched on

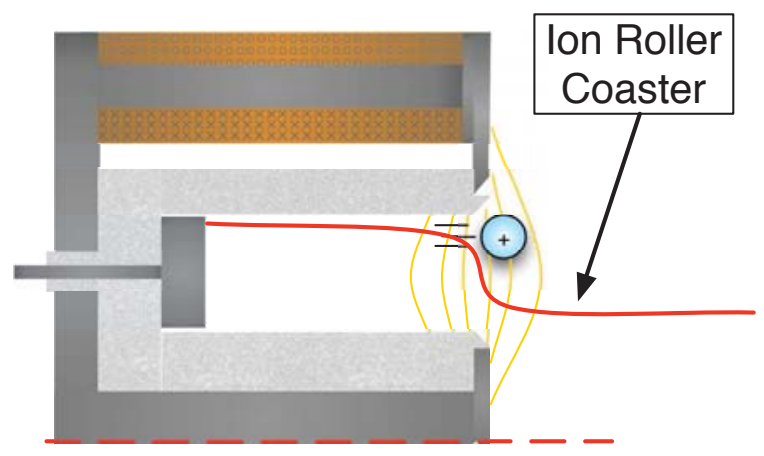

Figure 2.2: One quarter cross section of a Hall thruster. The yellow lines represent the radial magnetic field and the red line represents a typical plasma potential profile in the Hall thruster.

top of the thruster, serves to neutralize the ion beam that is ejected in order to prevent a build up of space charge. The electrons resulting from ionization (free electrons) and incident electrons (cathode electrons) are eventually collected at the anode. Before this, the electrons may be involved in many ionization events, but deposit any remaining energy in the form of joule heating of the anode.

\subsection{Probes}

\subsubsection{Retarding Potential Analyzer}

The retarding potential analyzer (RPA) is a tool used to measure the ion energyper-charge distribution of the plume of the Hall thruster [4]. Ultimately, this information is used to calculate voltage utilization efficiency (see section 2.3.1). The RPA probe has been used in countless studies in HET research $[5,6,7,8]$. In particular, the probe used here was also used in the several other studies $[9,10,11]$.

The RPA probe uses a series of biased grids to repel ions of low energy (see figures 2.3 and 2.4). The electron repelling grids (ER grids) are set to a fixed 

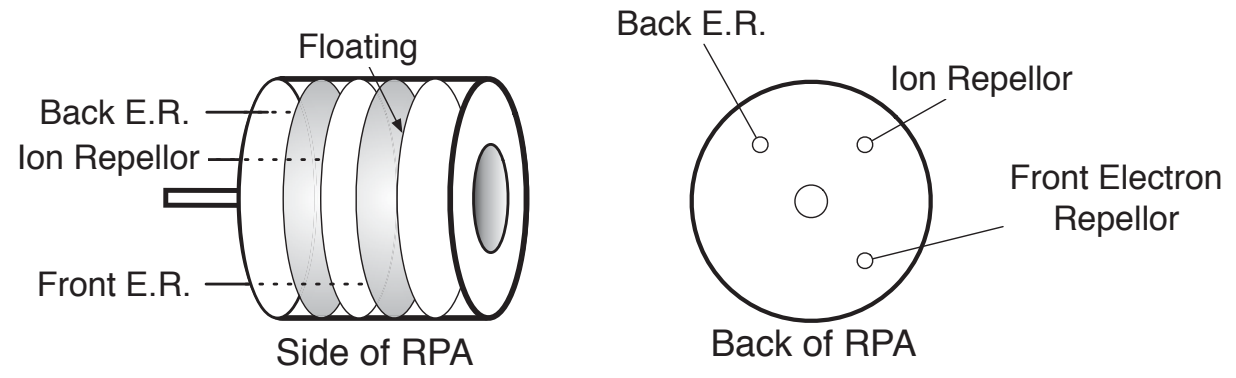

Figure 2.3: Internal diagram of the RPA probe where E.R. stands for electron repellor.

bias of $15 \mathrm{~V}$ below cathode potential and function to both suppress electrons from entering the probe, and to stop secondary electron emission off the collector in the back of the probe. The ion repeller is swept from $0 \mathrm{~V}$ to $450 \mathrm{~V}$ to filter out ions of low energy. The resulting I-V curve is the distribution function of ion energies (per charge). Since the RPA uses potential grids to filter the ions, it is not a direct measure of energy. In other words, a triple-charged charged xenon ion, $\mathrm{Xe}+++$, that reaches the collector, is indistinguishable from three single-charged ions. This quirk of the RPA is less of a concern for low voltage work because the fraction of multiply charged ions decreases with decreasing voltage [8].

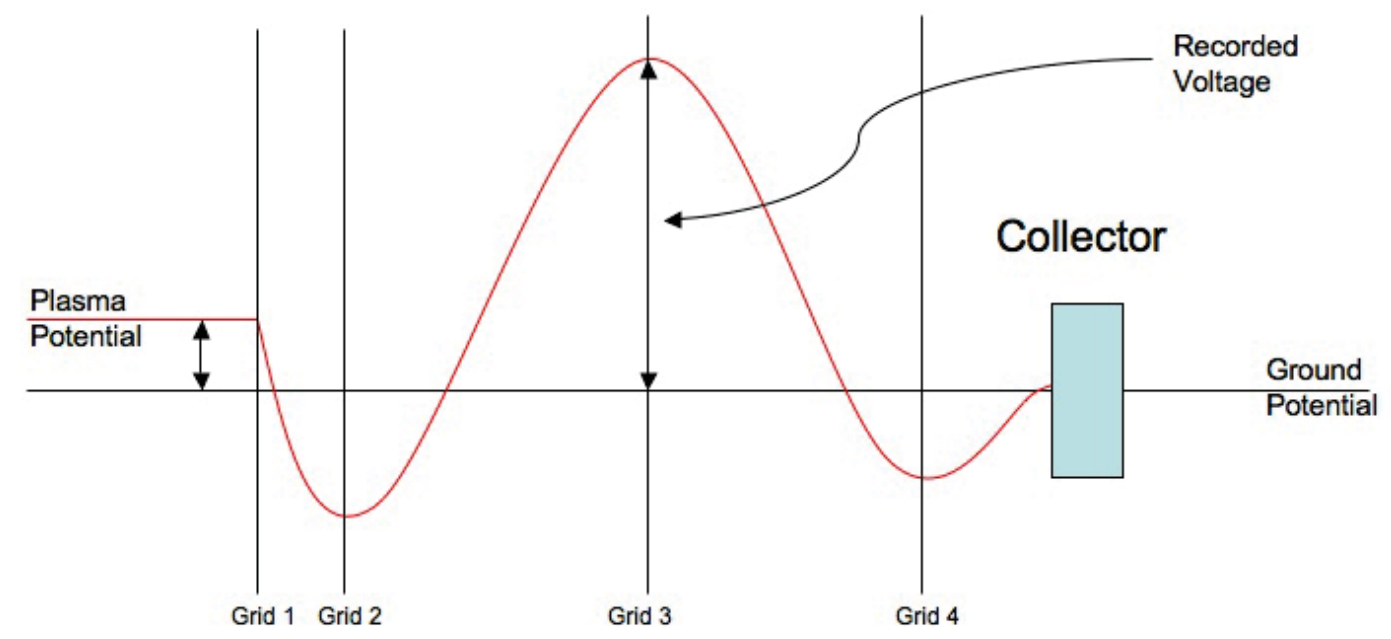

Figure 2.4: Potential structure created by the grids within the RPA 


\subsubsection{Faraday Probe}

A Faraday probe is a negatively biased electrode that collects ion current in the plume of a Hall thruster. By sweeping the probe in an arc in front of the thruster at a fixed bias, the current collected raises and lowers with the changing ion flux in the plume. From this data, current density, as a function of off-axis angle, can then be used to compute beam divergence efficiency (see section 2.3). The integration of the profile will also give beam current, $I_{B}$. The simple faraday probe has been used in the community for years, however, much advancement has been made in the technique in last year [12].

\subsubsection{Langmuir Probe}

Irving Langmuir first developed the fundamental techniques for determining plasma properties by the use of electrostatic probe in 1924 [13]. The single Langmuir probe consists of a piece of dielectric material, usually a refractory metal, housed in a ceramic body that is exposed on one end. The probe is connected to a power supply that sweeps the voltage applied to the probe and records the corresponding current to form an I-V trace from which the plasma properties can be extracted.

\section{Single Probe Interpretation}

The solid line in figure 2.5 depicts what an ideal Langmuir I-V curve from a single probe should look like. When the probe is biased very negatively, all of the

electrons are repelled and only the ions are collected. This represents the ion saturation region on the left side of the graph. As the potential on the probe is increased, eventually the current reaches zero, which, by definition, indicates floating potential. Further increasing the potential to the probe draws more electrons until eventually the probe potential reaches the the plasma potential, after which electron saturation occurs.

Ideally, plasma potential can be identified directly from the graph without further calculation, however, it is most often that in a single probe trace, electron saturation is never reached, and the knee in the curve is difficult to ascertain (see the doted line in figure 2.5). Furthermore, single probe operation requires the probe to be swept over a large range of voltages which in turn creates strong perturbations in the plasma rendering the results difficult to interpret correctly. This can be abated with multiple biased swept probes or a two-stage system in which the first sweep records the floating potential [14, 15]. Alternatively, a double Langmuir probe can be used. The double probe technique was chosen 


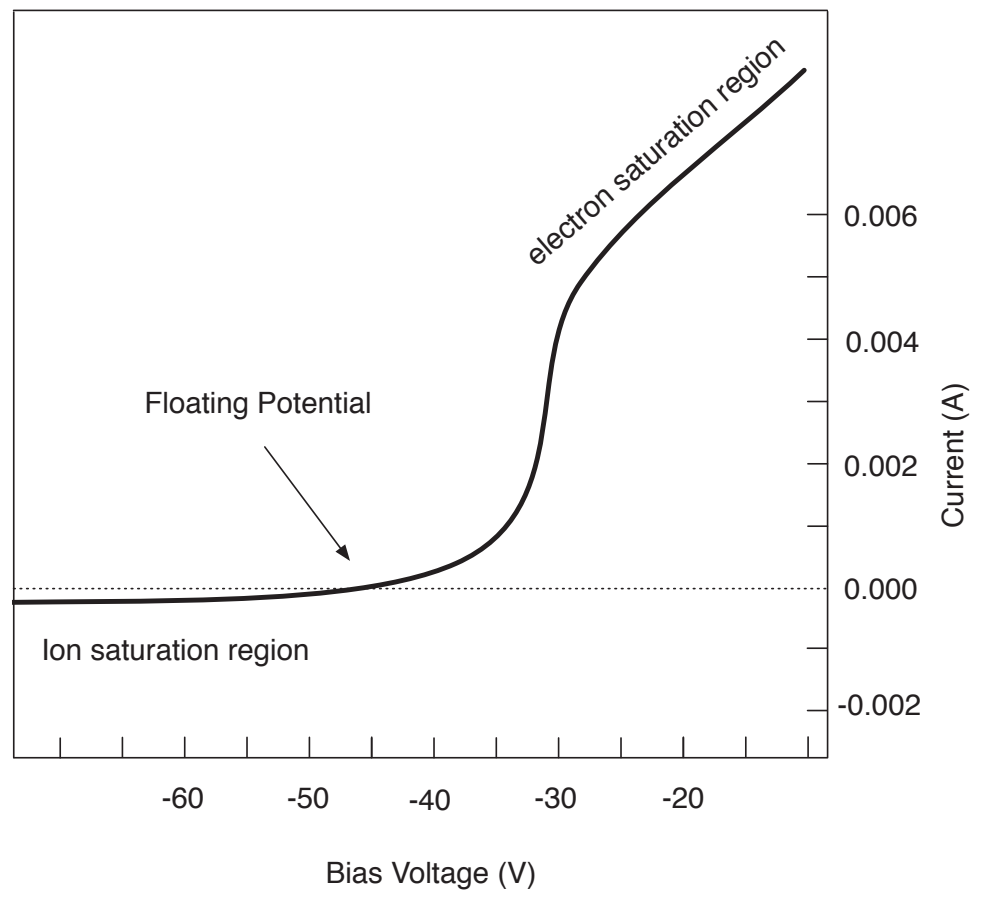

Figure 2.5: Ideal single Langmuir trace 
based on the recent experience and success with the double probe in the lab used for experimentation in this investigation $[9,16]$.

\section{Double Langmuir Probe}

The cylindrical double probe consists of two identical single probes mounted next to one another. A floating voltage supply creates a $\pm 40 \mathrm{~V}$ sweep between the probes and a ammeter records the current delivered to the probe tips. With the voltage supply floating, the potential half way between the two probes corresponds to the floating potential at the plasma location of the probe tips. This system has two benefits: First, the floating potential is easily acquired by measuring the the potential of the voltage supply with respect to ground, and second, neither probe is more than $\sim 40 \mathrm{~V}$ away from floating or plasma potential at any time, so the perturbation to the plasma is minimized.

\subsection{Thrust Efficiency}

The dominant gauge of performance of an EP device is the thrust efficiency. Thrust efficiency is calculated from thrust measurements taken from the thrust stand, power consumption measurements recorded from the power supply, and mass flow measurements from the controllers

$$
\eta_{T}=\frac{T^{2}}{2 \dot{m} P_{s} 4}
$$

Thrust efficiency is the measure of electrical power conversion to net thrust. This is, however, the product of many different processes in the Hall thruster. To diagnose loss mechanisms or to improve the efficiency ${ }^{1}$, these processes must be disentangled.

In 1998, Vladimir Kim presented a model to separate the thrust efficiency into five separate mathematical expressions, each relating to physical processes governing operation of the Hall thruster. Hofer built upon this structure, changing some of the terms and naming conventions in 2004 [8]. Most recently, however, Larson et al. have expanded this research to include even more of the physics of Hall thruster operation $[17,12]$. The efficiency breakdown here is a combination of both my own derivation and Larson's work, and covers the physics explored throughout the remainder of the dissertation.

The equation for thrust, eq.1.2, presented in chapter 1 was oversimplified. Thrust is the vector quantity

$$
T=\dot{m} \mathbf{V}
$$

\footnotetext{
${ }^{1}$ Anytime the word efficiency appears alone, it is referring to thrust efficiency.
} 
where $\mathbf{V}=U_{e} \hat{v}$. Substituting this into the thrust efficiency equation for this exploration gives

$$
\eta_{T}=\frac{\dot{m}^{2}\langle\mathbf{V}\rangle^{2}}{2 \dot{m} P_{s}}=\frac{\frac{1}{2} \dot{m}\langle\mathbf{V}\rangle^{2}}{P_{s}}=\frac{\langle\mathbf{V}\rangle^{2}}{\left\langle\mathbf{V}^{2}\right\rangle} \frac{\frac{1}{2} \dot{m}\left\langle\mathbf{V}^{2}\right\rangle}{P_{s}}
$$

This recast of the thrust efficiency equation breaks it into the product of propellant efficiency and energy efficiency. Propellant efficiency is the measure of the velocity distribution function and beam divergence efficiency

$$
\eta_{p}=\frac{\langle\mathbf{V}\rangle^{2}}{\left\langle\mathbf{V}^{2}\right\rangle} .
$$

Energy efficiency is the measure of total energy in the ion beam,

$$
\eta_{E}=\frac{\frac{1}{2} \dot{m}\left\langle\mathbf{V}^{2}\right\rangle}{P_{s}} .
$$

where

$$
P_{s}=I_{d} V_{d}
$$

as measured from the anode power supply.

Energy efficiency can be pulled apart further to get

$$
\eta_{E}=\frac{\frac{1}{2} m\left\langle\mathbf{V}^{2}\right\rangle}{e V_{d}} \frac{1}{f_{i} Q} \frac{\dot{m} e}{m I_{d}}\left(f_{i} Q\right)
$$

where $\mathrm{Q}$ is the average charge of the ionized propellant,

$$
Q=\frac{1}{f_{i}}\left(f_{1}+2 f_{2}+3 f_{3}\right) .
$$

The variable $f_{i}$ is the ionization mass fraction of the propellant

$$
\begin{gathered}
f_{i}=f_{1}+f_{2}+f_{3} \ldots \\
f_{0}+f_{i}=1
\end{gathered}
$$

where $f_{0}, f_{1}, f_{2}$, and $f_{3}$ are the exit mass fractions of $\mathrm{Xe}, \mathrm{Xe}^{+}, \mathrm{Xe}^{2+}$, and $\mathrm{Xe}^{3+}$. Thus, the quantity $f_{i} Q$ denotes average charge of the exhausted gas/plasma mix. This percentage accounts for exhausted neutrals as well as all multiple ionization species. The $f$ and $Q$ terms are pulled directly from the work of Larson [17]. 


\subsubsection{Voltage Utilization}

The first half of equation 2.12, is called the voltage utilization

$$
\eta_{v}=\frac{\frac{1}{2} m\left\langle\mathbf{V}^{2}\right\rangle}{e V_{d}} \frac{1}{f_{i} Q},
$$

where $\frac{1}{2} m\left\langle\mathbf{V}^{2}\right\rangle$ is the average energy of the expelled ions. The ions are homogenous in mass so by measuring their velocity we can calculate their energy. From there, the average portion of the electric potential that the ions have fallen through can be calculated

$$
\frac{1}{2} m U_{e}^{2}=q V_{\text {accel }}
$$

with the assumption that the acceleration voltage is equal to the discharge voltage applied to the anode, $V_{\text {accel }} \sim V_{d}$.

\subsubsection{Current Efficiency}

Current efficiency comes from the remaining half of equation 2.12

$$
\eta_{c}=\frac{\dot{m} e}{m I_{d}}\left(f_{i} Q\right)=\frac{I_{i}}{I_{d}}
$$

and measures the beam current versus the free electron current and ionization electron current.

\subsubsection{Velocity Distribution Function}

First, the propellent efficiency must be further separated into

$$
\eta_{p}=\frac{\langle|V|\rangle^{2}}{\left\langle|V|^{2}\right\rangle}\langle\cos \beta\rangle^{2}=\eta_{v d f} \eta_{B}
$$

to obtain the velocity distribution function (VDF)

$$
\eta_{v d f}=\frac{\langle|V|\rangle^{2}}{\left\langle|V|^{2}\right\rangle} .
$$

The velocity distribution function and the propellant efficiency are terms also adopted from Larson's work [17], which included examples of a VDF for a Gaussian and Maxwellian distribution. The VDF is the only efficiency that was not directly measurable by the used probe techniques used in this exploration. However, since thrust efficiency is known, the VDF is the quotient of $\eta_{T}$ divided by the other three loss mechanisms. In Larson's work, it was shown the the VDF is very small in comparison to the other loss mechanisms, and for this work has been considered negligible. 


\subsubsection{Beam Divergence}

Beam divergence is a measure of how collimated the ion beam is ejected at

$$
\eta_{B}=\langle\cos \beta\rangle^{2}
$$

A poorly structured magnetic field will allow the ions to escape the thruster at an angle not perpendicular to the exit plane. All off-axis trajectory components integrate out (for a symmetric beam) and are energy losses that do not contribute to thrust. There is also a pressure gradient due to high density plume that contributes to the beam spread, however, this loss is small in comparison to the effects of magnetic focusing.

\subsubsection{Thrust Efficiency Recombined}

Returning to the thrust efficiency, it can be depicted as the product of the velocity distribution function and the beam divergence, voltage utilization, and current efficiencies

$$
\eta_{T}=\eta_{v} \eta_{c} \eta_{v d f} \eta_{B}
$$

By measuring all of the individual efficiencies instead of just $\eta_{T}$, is is possible to uncover a deeper level of understanding of the underlying physics. In the following chapters, the probe techniques used to measure these efficiencies will be discussed. The role of the ionization potential as it relates to efficiency is presented in Appendix 3.4.

\subsection{Low Voltage Hall thrusters}

Nominal operating discharge voltages for Hall thrusters is 300-500 V. There has, however, recently been a push for high-voltage thrusters in the range of $1 \mathrm{kV}$. The term low voltage Hall thruster will be reserved for any thruster operating below $300 \mathrm{~V}$. Low voltage research is a vehicle to achieve high thrust to power thrusters. Unofficially, the electric propulsion community has set a target of $120 \mathrm{mN} / \mathrm{kW}$ thrust to power ratio. Figure 2.6 shows a good representation of the available Hall thrusters as of 2007, both research and production models. The highest level of thrust-to-power depicted in these models is just over $80 \mathrm{mN} / \mathrm{kW}$. Though more recent work has produced a thruster capable of the $86 \mathrm{mN} / \mathrm{kW}$, this value is still far beneath $120 \mathrm{mN} / \mathrm{kW}[12]$. 


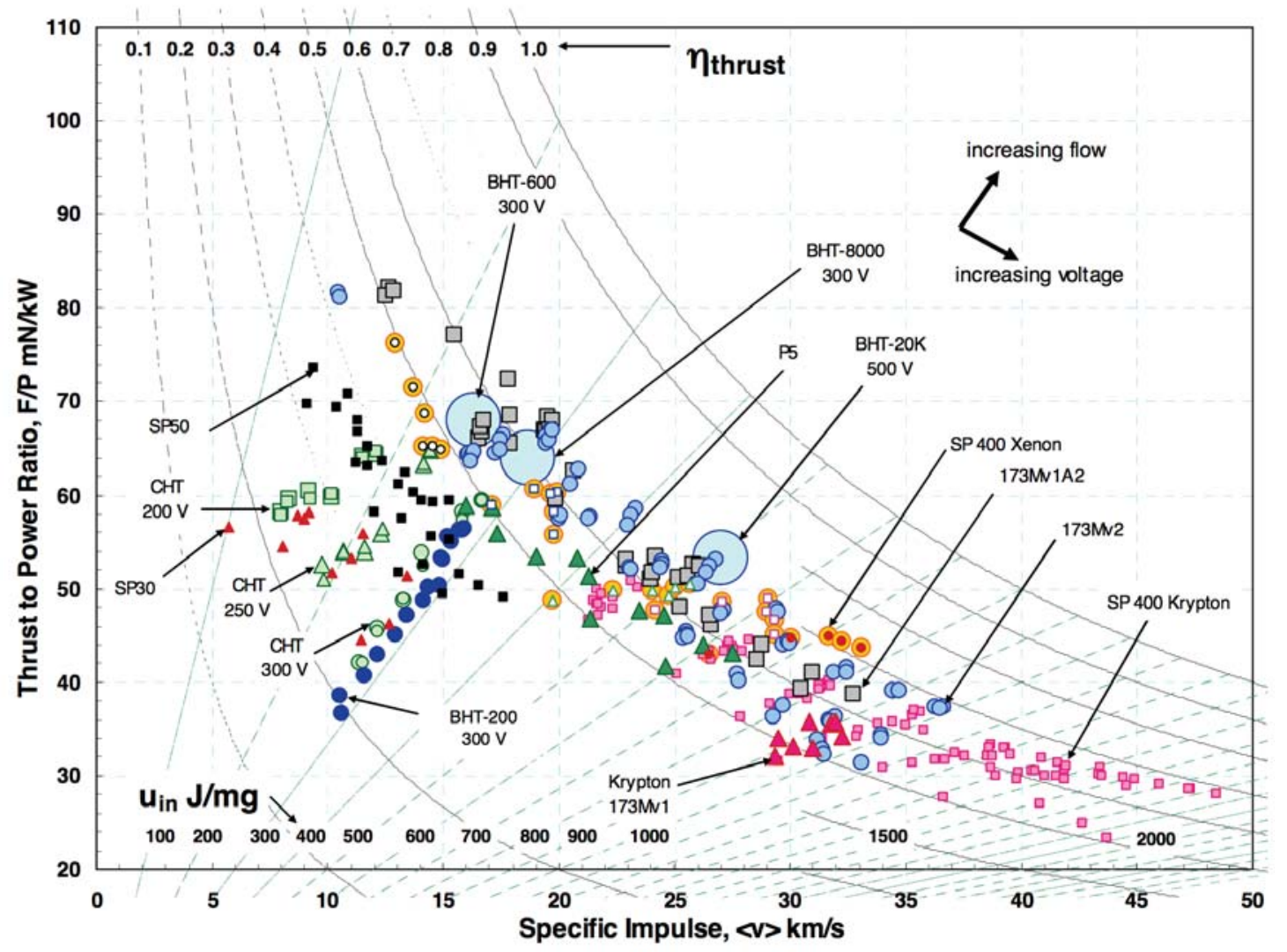

Figure 2.6: Performance characteristics of modern Hall thrusters in research and in industry. Larson, C. W., Brown, D. L., and Hargus, W. A., Thrust Efficiency, Energy Efficiency, and the Role of the VDF in Hall Thruster Performance Analysis, 43rd AIAA/ASME/SAE/ASEE Joint Propulsion Conference and Exhibit, Vol. AIAA 2007-5270, Cincinnati, Ohio, July 8-11, 2007 


\section{Industry Offerings}

Industry Hall thrusters available at low voltages include the Busek ${ }^{2}$ BHT-200 which operates at $250 \mathrm{~V}$ nominally at $200 \mathrm{~W}$ and Alta's XHT-100. Alta's thruster was created by scaling their $1 \mathrm{~kW}$ thruster to design the main propulsion system for a micro or minisatellite that operates nominally at $180 \mathrm{~V}$ and $100 \mathrm{~W}$ achieving only $22 \%$ efficiency[18]. Neither model is capable of high thrust to power but is geared for low power operation.

\subsection{Previous Research}

In 2005, TsNIIMASH reported $\sim 45 \%$ efficiency for a TAL (Thruster with Anode Layer) thruster operating at $200 \mathrm{~V}$ and $3.5 \mathrm{mg} / \mathrm{s}$ flow of xenon for a thrust to power ratio of $73 \mathrm{mN} / \mathrm{kW}$. They further demonstrated that the power and thrust can be linearly scaled with thruster arrays or clusters (maintaining the same thrust-to-power ratio as an individual thruster) [19]. Clusters also add mass and complexity to the system that could be alleviated by a single thruster with identical capabilities. Though, this approach does have the benefit of redundancy.

Research has shown that alternative propellants such as bismuth lower the cost of ionization, a power loss aggravated at low discharge voltages [3, 20]. Additionally, as a heavier atom than xenon, bismuth would also decrease the exit velocity for a given discharge voltage according to Eq. 2.14. Performance data for bismuth Hall thrusters, however, are not yet available. Also, using alternative propellants will benefit high thrust-to-power operation, but does not address the physics inhibiting high efficiency at low discharge voltages.

In 2003, Manzella experimented on the NASA-120M and NASA-457M Hall thrusters and narrowed the leading loss mechanism to voltage utilization efficiency or ionization efficiency of low voltage operation. The devices in the aforementioned study were operated as low as $100 \mathrm{~V}$ on the anode at $<25 \%$ efficiency[21]. The study achieved upwards of $80 \mathrm{mN} / \mathrm{kW}$ with the larger of the two thrusters the 457M. The data included thrust measurements, mass flow settings, and discharge power supply readings. With this information, the ionization fraction and exit velocity are indeterminate. Hence, the loss mechanisms can only be postulated without probe data.

Referring back to figure 2.6, the top two performers in the thrust to power category were the 173Mv1 and Mv2 in the work done by Hofer (2002-2004) [8, $22,5]$. This work, however, was focused on high discharge voltage data ranging from $300-1000 \mathrm{~V}$. The superior magnetic field design benefited the thrusters

\footnotetext{
${ }^{2}$ http://busek.com/halleffect.html\#low
} 
efficiency and thus thrust-to-power ratio. Results for voltages below $300 \mathrm{~V}$ were not published.

The most recent and most relevant work in low voltage Hall thrusters was performed by Dan Brown. Brown used a $6 \mathrm{~kW}$ laboratory thruster to perform exhaustive probe and thrust stand measurements for discharge voltages of 300 $\mathrm{V}$ down to $105 \mathrm{~V}$ at both $20 \mathrm{mg} / \mathrm{s}$ and $10 \mathrm{mg} / \mathrm{s}$ propellant flow rates. At 20 $\mathrm{mg} / \mathrm{s}$, Brown showed a maximum thrust-to-power of $86.4 \mathrm{mN} / \mathrm{kW}$ at a discharge voltage of $120 \mathrm{~V}$. To date, this is the largest published thrust-to-power ratio in the community literature. At $100 \mathrm{~V} V_{d}$ the ratio dropped as the thrust efficiency fell from $41 \%$ to $34 \%$. The recycled electron current was the dominant loss mechanism at $71 \%$ current efficiency. The voltage utilization efficiency, however, saw the greatest change, $-14 \%$, when the discharge voltage was reduced from the nominal $300 \mathrm{~V}$ down to $105 \mathrm{~V}$.

In the $10 \mathrm{mg} / \mathrm{s}$ case, the maximum thrust-to-power was $85.5 \mathrm{mN} / \mathrm{kW}$ and the current efficiency was also the dominant loss mechanism. Here, current efficiency exhibited the greatest change, $-16 \%$, from the nominal discharge voltage at this flow rate. All measurements in all test cases were taken at maximum thrust efficiency for a given discharge voltage.

\subsection{Contribution}

The research herein bolsters the experimental data for Hall thrusters operating at low voltages. As previously noted, there are unfortunately very few studies aimed at understanding the link between low voltage operation and the poor thrust efficiency that results. Brown's dissertation work took great strides in this arena, and it is the goal of this work to make an additional contribution. Specifically, this experiment utilizes internal probe data that allow for a more in depth understanding of the acceleration and ionization processes seen in the low voltage thrusters. To date, internal probe studies have only been performed on nominal to high voltage Hall thrusters. A traditional set of external probe measurements and thrust stand studies are also included in this dissertation as a means of comparison against the internal data and serve as a benchmark for overall operation of the Hall thruster examined. Another unique facet of this work is the inclusion of off peak thrust efficiency measurements. In typical operation, a Hall thruster is tuned to maximum thrust efficiency as controlled by the proper tuning of the magnet coil current, and there is little reason to investigate off-peak characteristics. In low voltage operation, it will be shown that several of the staple behavioral operating characteristics differ from nominal operation. As such, it is believed that new insights in the peculiarities of low voltage operation might be brought into light with a wider envelope of testing characteristics. Off-peak thrust 
efficiency probe studies such as this are a rarity in the field. 


\section{Bibliography}

[1] Jahn, R. G., Physics of Electric Propulsion, Dover Publications, 1968.

[2] Kaufman, H. R., "Technology of Closed-Drift Thrusters," AIAA Journal, Vol. 23, No. 1, 1985.

[3] Massey, D. R., King, L. B., and Makela, J. M., "Progress on the Development of a Direct Evaporation Bismuth Hall Thruster," 29th International Electric Propulsion Conference, Vol. IEPC-2005-256, Princeton, New Jersey, October 31 - November 42005.

[4] Hutchinson, I. H., Principles of Plasma Diagnostics, Cambridge University Press, 1987.

[5] Hofer, R. R., Development and Characterization of High-Efficiency, HighSpecific Impulse Xenon Hall Thrusters, Ph.D. thesis, University of Michigan, Ann Arbor, 2004.

[6] Kieckhafer, A., The Effect of Segmented Anodes on the Performance and Plume of a Hall Thruster, Ph.D. thesis, Michigan Technological University, 2007.

[7] King, L. B., Transport-Property and Mass Spectral Measurements in the Plasma Exhaust Plume of a Hall-Effect Space Propulsion System, Ph.D. thesis, University of Michigan, 1998.

[8] Hofer, R. R. and Gallimore, A. D., "Efficiency Analysis of a High-Specific Impulse Hall Thruster," 40th AIAA/ASME/SAE/ASEE Joint Propulsion Conference \& Exhibit, Vol. AIAA-2004-3602, Ft. Lauderdale, Florida, July 11-14 2004.

[9] Sommerville, J. D., Hall-Effect Thruster-Cathode Coupling: The Effect of Cathode Position and Magnetic Field Topology, Ph.D. thesis, Michigan Technological University, 2009. 
[10] Ross, J. L. and King, L. B., "Energy Efficiency in Low Voltage Hall Thrusters," 43rd AIAA/ASME/SAE/ASEE Joint Propulsion Conference \&6 Exhibit, Vol. AIAA-2007-5179, Cincinnati, OH, July 8-11 2007.

[11] Ross, J. L., Sommerville, J. D., and King, L. B., "Energy-Loss Mechanisms of a Low-Discharge-Voltage Hall Thruster," Journal of Propulsion and Power, Vol. 26, No. 6, Nov.-Dec. 2010, pp. 1312-1317.

[12] Brown, D. L., Investigation of Low Discharge Voltage Hall Thruster Characteristics and Evaluation of Loss Mechanisms, Ph.D. thesis, University of Michigan, June 2009.

[13] Chen, F. F., Plasma Diagnostic Techniques, Academic Press, New York, NY, 1965.

[14] Linnell, J. A. and Gallimore, A. D., "Internal Langmuir Probe Mapping of a Hall Thruster with Xenon and Krypton Prop," Vol. AIAA-2006-4470, Sacramento, California, 9-12 July 2006.

[15] Reid, B. M. and Gallimore, A. D., "Langmuir Probe Measurements in the Discharge Channel of a 6-kW Hall Thruster," 44th AIAA/ASME/SAE/ASEE Joint Propulsion Conference and Exhibit, Vol. AIAA-2008-4920, Hartford, CT, July 20-23 2008.

[16] Washeleski, R. L. and King, L. B., "Characterization of the Plasma Plume form a Lab6 Cathode: A Comparison of Probe Techniques," 45th AIAA/AS$M E / S A E / A S E E$ Joint Propulsion Conference and Exhibit, Denver, Colorado, August 2-5 2009.

[17] Larson, C. W., Brown, D. L., and Hargus, W. A., "Thrust Efficiency, Energy Efficiency, and the Role of the VDF in Hall Thruster Performance Analysis," 43rd AIAA/ASME/SAE/ASEE Joint Propulsion Conference and Exhibit, Vol. AIAA 2007-5270, Cincinnati, Ohio, July 8-11 2007.

[18] Andrenucci, M., Berti, M., Biagioni, L., and Cesari, U., "Characteristics of the XHT-100 Low Power Hall Thruster Prototype," 4th International Spacecraft Propulsion Conference, Vol. ESA SP-555, Chia Laguna, Sardinia, Italy, October 2004.

[19] Zakharenkov, L. E., Semenkin, A. V., and Garkusha, V. I., "Study of the 3TAL Thruster Assembly Operation," 29th International Electric Propulsion Conference, Vol. IEPC-2005-185, Princeton, NJ, October 31 - November 4 2005. 
[20] Kieckhafer, A. and King, L. B., "Energetics of Propellant Options for HighPower Hall Thrusters," 41st AIAA/ASME/SAE/ASEE Joint Propulsion Conference and Exhibit, Vol. AIAA-2005-4228, Tucson, Arizona, July 10-13 2005.

[21] Manzella, D. and Jacobson, D., "Investigation of Low-Voltage/High-Thrust Hall Thruster Operation," 39th AIAA/ASME/SAE/ASEE Joint Propulsion Conference and Exhibit, Vol. AIAA-2003-5004, Huntsville, Alabama, July 20-23 2003.

[22] Hofer, R. R. and Gallimore, A. D., "High-Specific Impulse Hall Thrusters, Part 2: Efficiency Analysis," Journal of Propulsion and Power, Vol. 07484658, No. 4, July-August 2006, pp. 732-740. 


\section{Chapter 3}

\section{Loss Mechanisms as a Function of Discharge Voltage}

This first experiment was a parametric study of an commercial grade Hall thruster

operated at low voltages. The goal of this work was to create benchmark of operation for the BPT-2000 as a low voltage thruster by implementing a variety of thrust stand and probe studies.

The study was broken up into two parts: First, the thruster was mounted on a thrust stand where, in addition to thrust measurements, power consumption and mass flow data were recorded. Secondly, the thrust stand was removed and the Hall thruster was rigidly mounted while two probe diagnostics were swept through the subset of the thrust stand test matrix. The two probe diagnostic tools were a Faraday probe and a retarding potential analyzer (RPA). The results of the two stages of the experiment were combined to provide thrust, beam divergence, current, and voltage utilization efficiencies.

\subsection{Equipment \& Facilities}

\subsubsection{BPT-2000 Hall Thruster}

All measurements in this dissertation were taken on an Aerojet BPT-2000 a 2kW class Hall thruster designed to operate nominally between $300 \mathrm{~V}$ and $500 \mathrm{~V}$ [1]. Previous work with this thruster was the subject of numerous other studies $[2,3$, $4,5,6,7]$. 


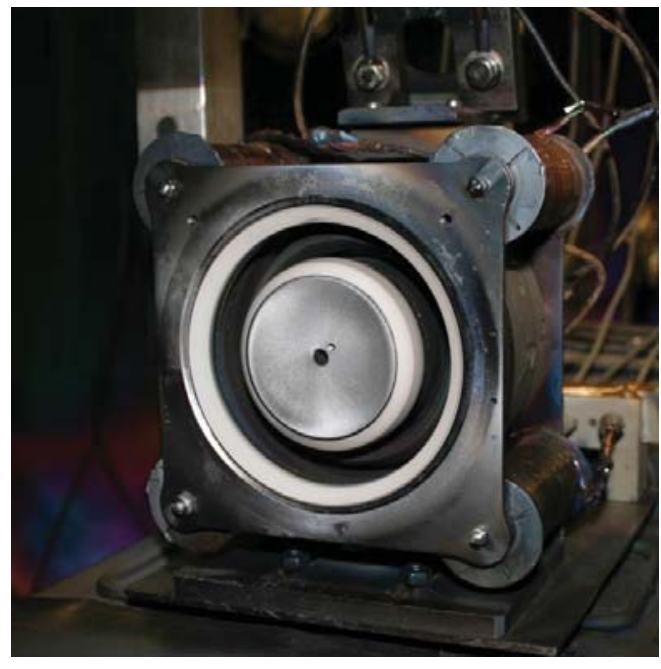

(a) Thruster Off

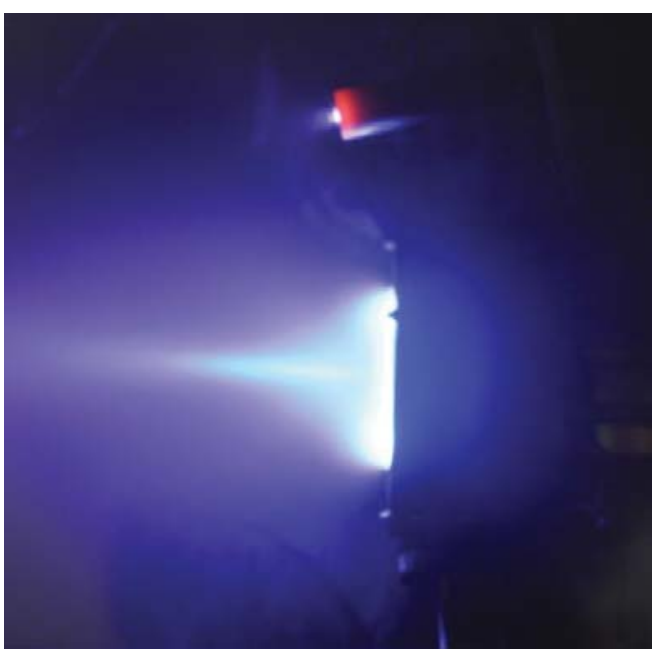

(b) Thruster Operating on Krypton

Figure 3.1: The Aerojet BPT-2000 Hall thruster

\subsubsection{Cathode}

A laboratory-grade $\mathrm{LaB}_{6}$ cathode was used for testing at a constant flow of 0.3 $\mathrm{mg} / \mathrm{s}$ xenon. All efficiency values, both referenced and presented, exclude cathode flow and heater power. The details of cathode and its construction have been in several other studies [3].

\subsubsection{Vacuum Tank and Pumps}

The xenon testing facility is a 2-m diameter and 4-m long vacuum chamber. The thruster is mounted at the radial center $1 \mathrm{~m}$ from the end of the tank (see Figure $3.2)$.

Rough vacuum is reached by a 400-cfm two-stage rotary oil-sealed pump. High vacuum is reached and maintained by two 48-inch cryopumps that operate at $120,000 \mathrm{~L} / \mathrm{s}\left(\mathrm{N}_{2}\right)$. Chamber base pressure was $1 \times 10^{-6}$ Torr, and the pressure did not exceed $4 \times 10^{-5}$ Torr (corrected for xenon) during thruster operation.

\subsubsection{Thrust Stand and Mass Flow Controllers}

Thrust measurements were taken via an inverted-pendulum thrust stand [8]. The displacement created by the Hall thruster is recorded by a linear voltage displacement transducer (LVDT). Details of the thrust stand can be found in Sommerville's dissertation [3]. The thrust stand is water cooled to alleviate thermal 


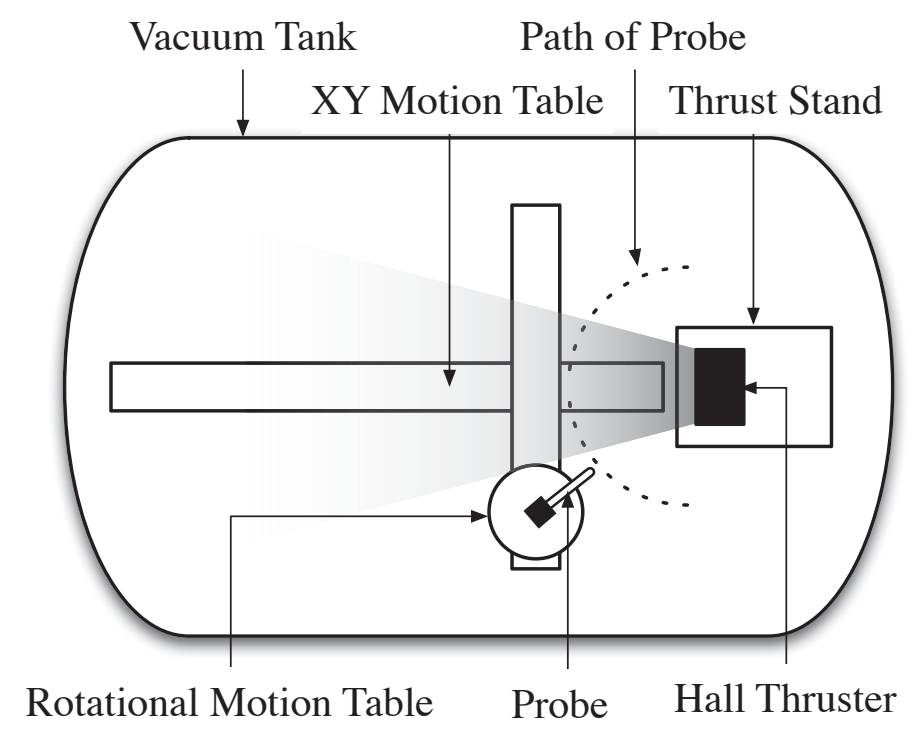

Figure 3.2: A top down view of the inside of the xenon Hall thruster testing facility

drifts and its level is monitored by a tilt sensor accurate to one half an arc second. The thrust stand was calibrated before each change in mass flow rate.

Anode and cathode propellant feeds are controlled by thermal mass flow controllers that used MKS PR-4000's as front panels. All flow rates were corrected for xenon.

\begin{tabular}{ccc} 
Feed Line & Model & Maximum Flow \\
\hline Anode & MKS - 1179A22CS1BV & 200 SCCM \\
Cathode & MKS - 1479A21CS1AM & 20 SCCM
\end{tabular}

Table 3.1: Mass flow system 
Table 3.2: Thrust stand testing matrix. Many of the lower voltages were not recorded for 4 and $5 \mathrm{mg} / \mathrm{s}$.

\begin{tabular}{|c|c|}
\hline Anode Voltage $(\mathrm{V})$ & Mass Flow Rate $(\mathrm{mg} / \mathrm{s})$ \\
\hline 300 & 5 \\
275 & 5 \\
250 & 5 \\
225 & 5 \\
200 & 5 \\
180 & 5 \\
300 & 4 \\
275 & 4 \\
250 & 4 \\
225 & 4 \\
200 & 4 \\
180 & 4 \\
160 & 4 \\
140 & 4 \\
300 & 3 \\
275 & 3 \\
250 & 3 \\
225 & 3 \\
200 & 3 \\
180 & 3 \\
160 & 3 \\
140 & 3 \\
120 & 3 \\
100 & 3 \\
\hline
\end{tabular}




\subsection{Testing Matrices}

\subsubsection{Thrust Stand Experiment}

Table 3.2 shows the operating conditions used in the thrust stand portion of the experiment. The Hall thruster was only able to complete entire $300 \mathrm{~V}-100 \mathrm{~V}$ test range when operating at $3 \mathrm{mg} / \mathrm{s}$ flow rate. At the higher mass flow rates the thruster ran unstably or not at all in most cases.

At each operating condition, the magnets were adjusted to obtain the maximum thrust efficiency as calculated by thrust stand diagnostics.

\subsubsection{Probe Study Experiment}

Due to the volume of data to be collected, the probe study test matrix is a subset of the thrust stand test matrix.

Table 3.3: Probe study testing matrix. The probe study was conducted over a subset of the thrust stand testing matrix.

\begin{tabular}{|c|c|}
\hline Anode Voltage (V) & Mass Flow Rate $(\mathrm{mg} / \mathrm{s})$ \\
\hline 300 & 5 \\
250 & 5 \\
200 & 5 \\
300 & 4 \\
250 & 4 \\
200 & 4 \\
140 & 4 \\
300 & 3 \\
250 & 3 \\
200 & 3 \\
140 & 3 \\
100 & 3 \\
\hline
\end{tabular}

\subsection{Retarding Potential Probe}

The RPA grid wires are $0.114 \mathrm{~mm}$ in diameter with $0.140 \mathrm{~mm}$ spacing resulting in a $30 \%$ open area. Each grid is $0.254 \mathrm{~cm}$ from each other with the exception of the front floating grid which is $0.508 \mathrm{~cm}$ from the first electron repeller. The outer diameter of the grids is $1.235 \mathrm{~cm}$, and the outer diameter of the body of 
the probe is $3.170 \mathrm{~cm}$. The current collected by the probe was passed through a Femto DLCPA-200 current amplifier and recorded by an oscilloscope. The RPA probe was placed $0.55 \mathrm{~m}$ downstream from the thruster faceplate at $0^{\circ}, 15^{\circ}$, and $30^{\circ}$ to the thruster centerline. Figure 3.3 is a picture of both the Faraday probe and the RPA probe mounted on the rotational axis.

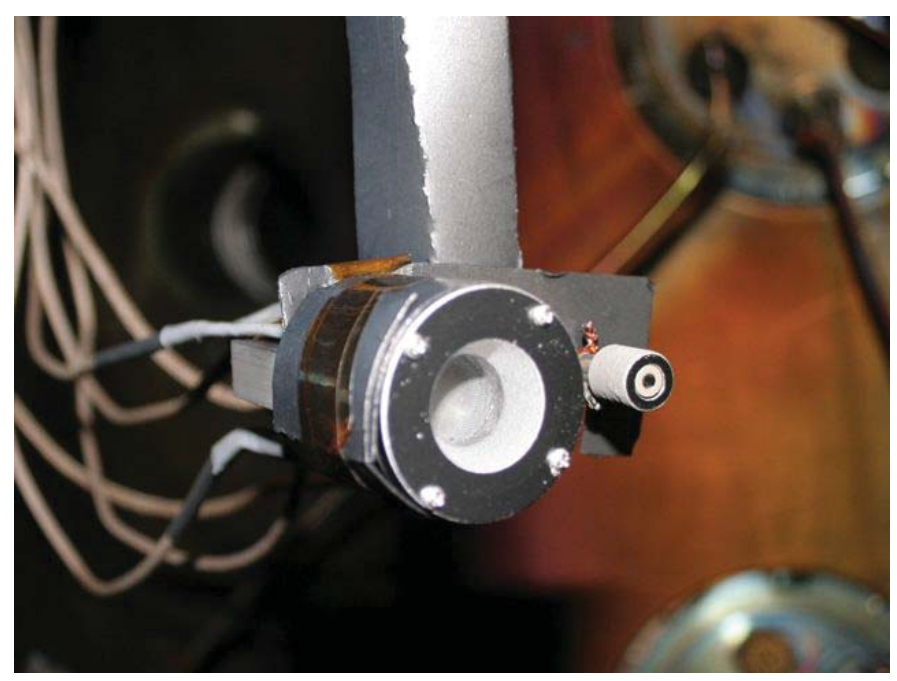

Figure 3.3: RPA probe on the left and Faraday Probe on the right

\section{RPA Referencing}

The anode and RPA were both referenced to the cathode potential during data acquisition. In calculating the voltage utilization efficiency, the repelling voltages were adjusted to be referenced to ground potential. The ion-energy per charge distribution is dependent on the local plasma potential at the position of the probe. Even though the retarding grid is referenced to cathode, the energy acquired by the ions is equal to the potential drop between the discharge potential and the local plasma potential. In the absence of the probe, the ions will continue to accelerate until they reach their terminal potential, which, in the case of ground testing, is the tank wall (ground) potential. Hence, RPA measurements are sensitive to the location of the probe. Thus, to remove the positional dependence of the RPA, knowledge of both the local plasma potential and cathode-to-ground potential is necessary. Assuming that the RPA grids are referenced to ground the energy values can be corrected by

$$
\left\langle\frac{\epsilon_{i o n}}{q}\right\rangle=\left\langle\frac{\epsilon_{r p a}}{q}\right\rangle-\Phi_{c t g}+\Phi_{\text {plasma }}
$$


where the charge number is assumed to be unity and on the left hand side is the ion energy to be used in the correct calculation of Eq. 2.13.

RPA traces were taken at $0^{\circ}, 15^{\circ}$, and $30^{\circ}$, and average ion energy values were linearly interpolated for angles in between $0^{\circ}$ and $15^{\circ}$ and in between $15^{\circ}$ and $30^{\circ}$. The energy values from $30^{\circ}$ out to $53^{\circ}$ were assumed to be constant. While the validity of this extrapolation is admittedly unknown, past studies indicate the approach is reasonable. King's RPA results on a $1.5 \mathrm{~kW}$-class Hall thruster showed that measured probe values varied less than $5 \%$ from $30^{\circ}$ to $60^{\circ}$ [9]. An assumption of $5 \%$ error in the high-angle extrapolated probe values propagates through the integral as $2 \%$ uncertainty in the calculation of Eq. 3.2. Finally, the traces are weighted against the current distribution function from the Faraday data and integrated to obtain the overall voltage utilization efficiency. Thus, by equation 3.2 the final values for $\eta_{V}$ are obtained and are shown in figures 7.1 to 7.3 .

$$
\eta_{V}=\frac{\int \epsilon_{i o n}(\theta) j(\theta) r^{2} \sin \theta d \theta}{q e V_{d} \int j(\theta) r^{2} \sin \theta d \theta}
$$

\subsection{Faraday Probe}

The Faraday probe is enclosed in an alumina sheath with an outer diameter of $4.75 \mathrm{~mm}$. A steel guard ring with a diameter of $10 \mathrm{~mm}$ was included to reduce

edge effects on the potential structure in front of the probe face. The gap between the guard ring and collector face is $1.25 \mathrm{~mm}$. Probe data were taken at $0.25 \mathrm{~m}$ radius from the front plate of the thruster through a half-angle of $53^{\circ}$ with an angular resolution of $2^{\circ}$. Figure 3.4 shows an example of current density recorded by the Faraday probe for five different anode voltages and a flow rate of $3 \mathrm{mg} / \mathrm{s}$.

To calculate the beam divergence efficiency, $\eta_{B}$, from the raw Faraday data, the current density values, $j(\theta)$, must be integrated across the hemisphere representing the entire plume of the thruster, as shown in equation 3.3.

$$
\eta_{B}=\langle\cos \beta\rangle^{2}=\left(\frac{\int \cos \theta j(\theta) r^{2} \sin \theta d \theta}{\int j(\theta) r^{2} \sin \theta d \theta}\right)^{2}
$$

\subsection{Results}

\subsubsection{Thrust Efficiency}

The thrust stand measurements in figure 3.5 show the thruster operated at above $50 \%$ thrust efficiency for all three mass flow rates at $300 \mathrm{~V}$. The data here is 


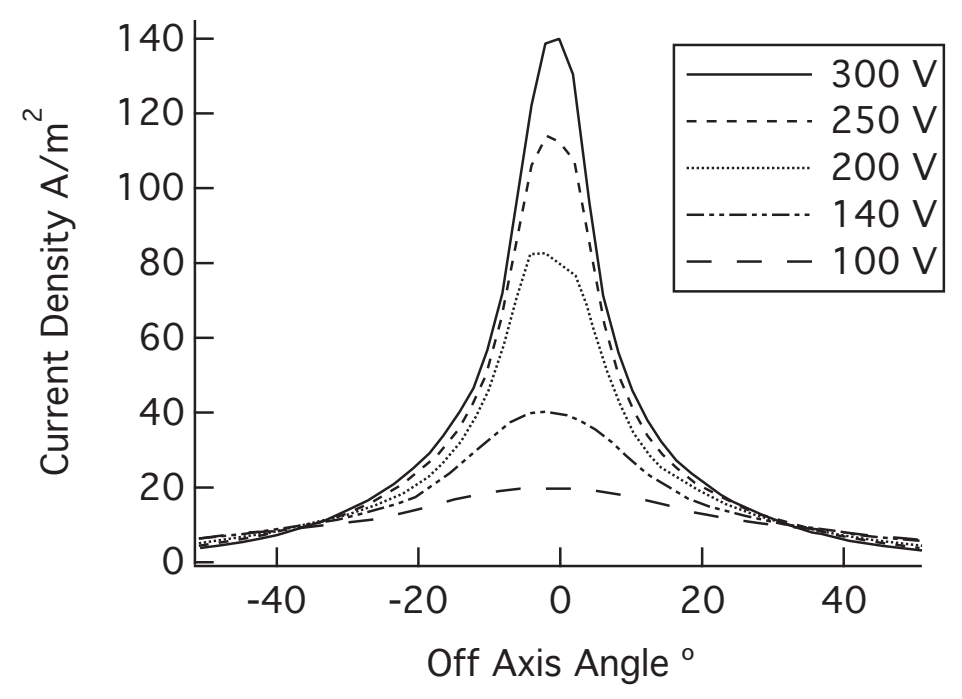

Figure 3.4: Faraday probe sweeps $250 \mathrm{~mm}$ downstream for $3 \mathrm{mg} / \mathrm{s}$ flow rate at five different discharge voltages

comparable with the data from King after the cathode operation is accounted for[1]. ${ }^{1}$ At $V_{d}=100 \mathrm{~V}$, maximum thrust efficiency was $15 \%$ for $3 \mathrm{mg} / \mathrm{s}$.

\section{Discharge Current}

The discharge current was also measured during the thrust stand portion of the experiment. The results are plotted against the discharge voltage in Figure 3.6. The current levels were nearly constant for each mass flow rate until the discharge voltage dropped below $200 \mathrm{~V}$ where the current increased rapidly; again, this increase in current is the result of the magnetic field being optimized for maximum $\eta_{T}$.

\section{Thrust-to-Power ratio}

Taking the thrust measurements from Figure 3.5 and dividing by the product of the anode voltage and discharge current results of Figure 3.6, we get the thrustto-power ratio, plotted in Figure 3.7. Although the thrust-to-power ratio does increase when the voltage decreases from the nominal $300 \mathrm{~V}$ to $250 \mathrm{~V}$, it is by

\footnotetext{
${ }^{1}$ For the $300 \mathrm{~V}$ data. Data for discharge voltage lower than $300 \mathrm{~V}$ was not available for comparision.
} 


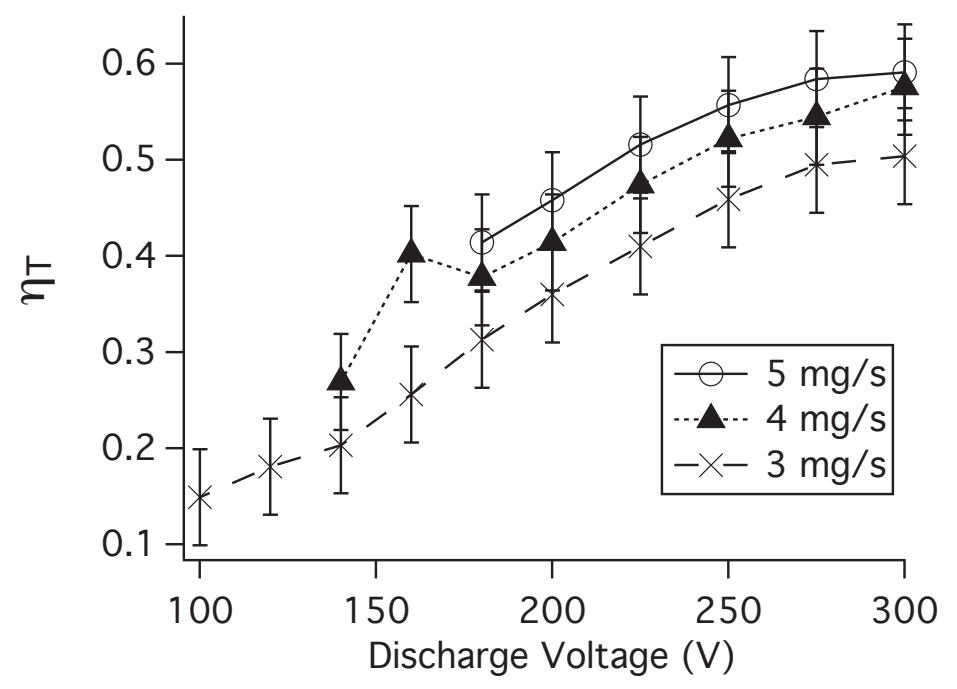

Figure 3.5: Thrust efficiency as determined by thrust measurements. Error bars are calculated based on the reproducibility of calibration measurements both during operation and when the thruster is turned off.

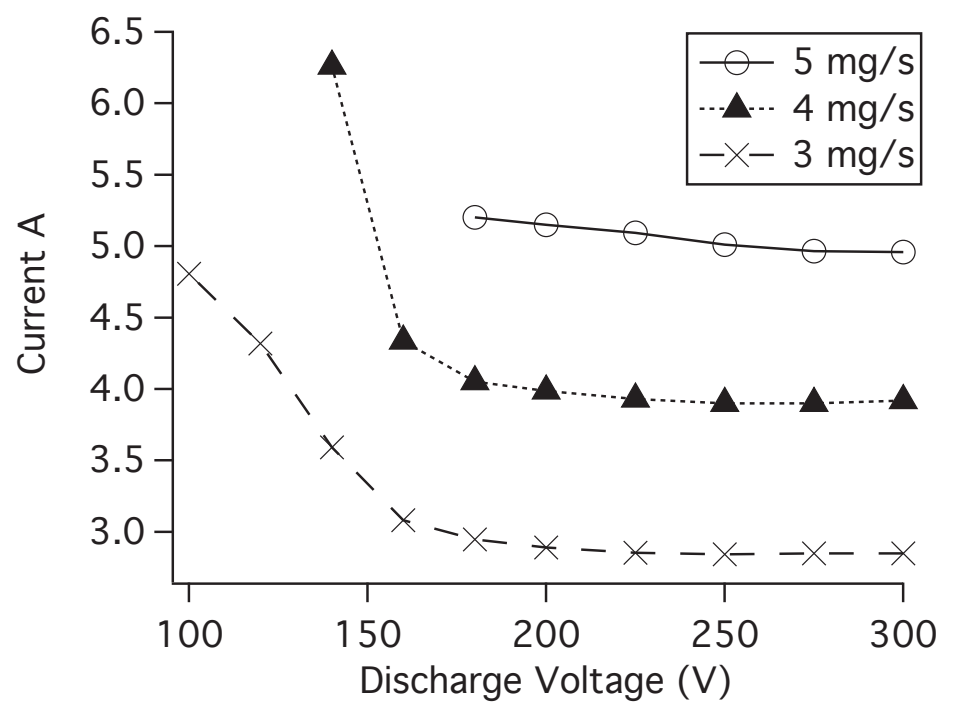

Figure 3.6: Discharge current at $\max \eta_{T}$. Uncertainty is $\pm 0.05 \mathrm{~A}$ based on manufacturer's specified uncertainty for the discharge power supply. 


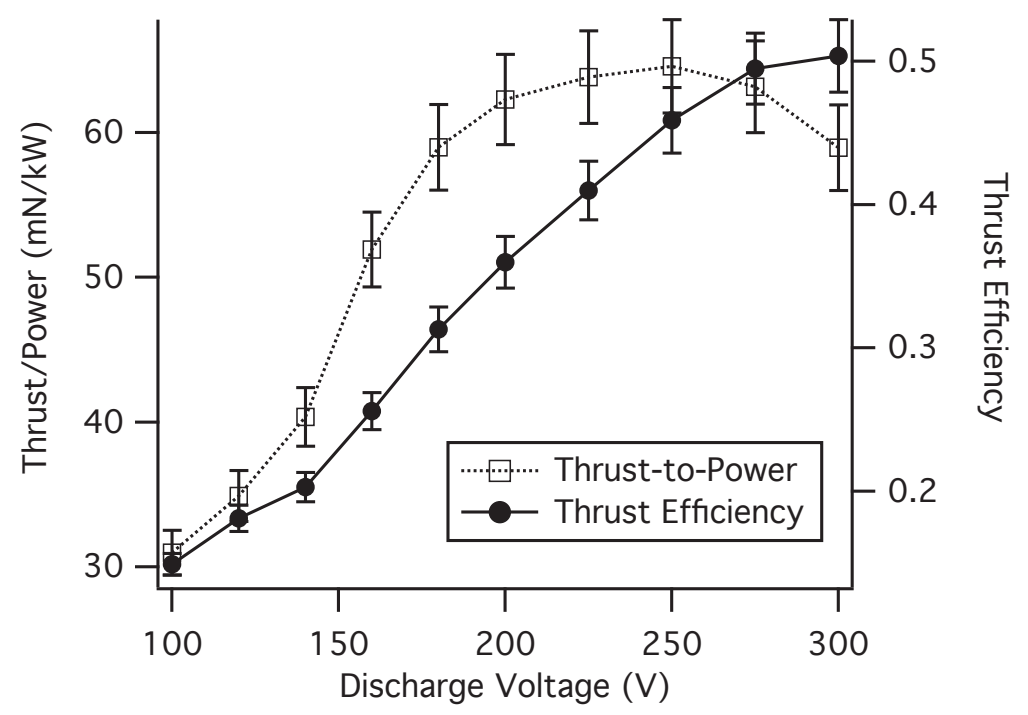

Figure 3.7: Thrust to Power ratio for the BPT-2000 operating on 3 $\mathrm{mg} / \mathrm{s}$ of xenon.

less than 10\%. More importantly, the ratio begins to decrease monotonically afterwards. This will be examined in greater detail in the discussion chapter.

\subsubsection{Voltage Utilization Efficiency}

Figures 3.8 - 3.10 display the integrated ion-energy per charge distributions for three off-axis angles. The greatest change in efficiency occurred in the $3 \mathrm{mg} / \mathrm{s}$ experiments where the voltage utilization efficiency dropped from $76 \%$ at $300 \mathrm{~V}$ to $54 \%$ at $100 \mathrm{~V}$ when the probe was positioned $30^{\circ}$ off-axis. As seen with other Hall thrusters, the voltage utilization efficiency decreases with increasing off axis angle[9]. The dominant error in figures 3.8 to 3.10 came from the uncertainty in the integrated ion-energy per charge distributions due to noise in the recorded $\delta I / \delta V$ trace.

\subsubsection{Beam Divergence Efficiency}

Using equation 3.3, Figure 3.11 was calculated with the assumption that the plume is axisymmetric. As a result, beam divergence efficiency is subject to underestimation based on the positioning of the Faraday probe. An accurate sweep would 


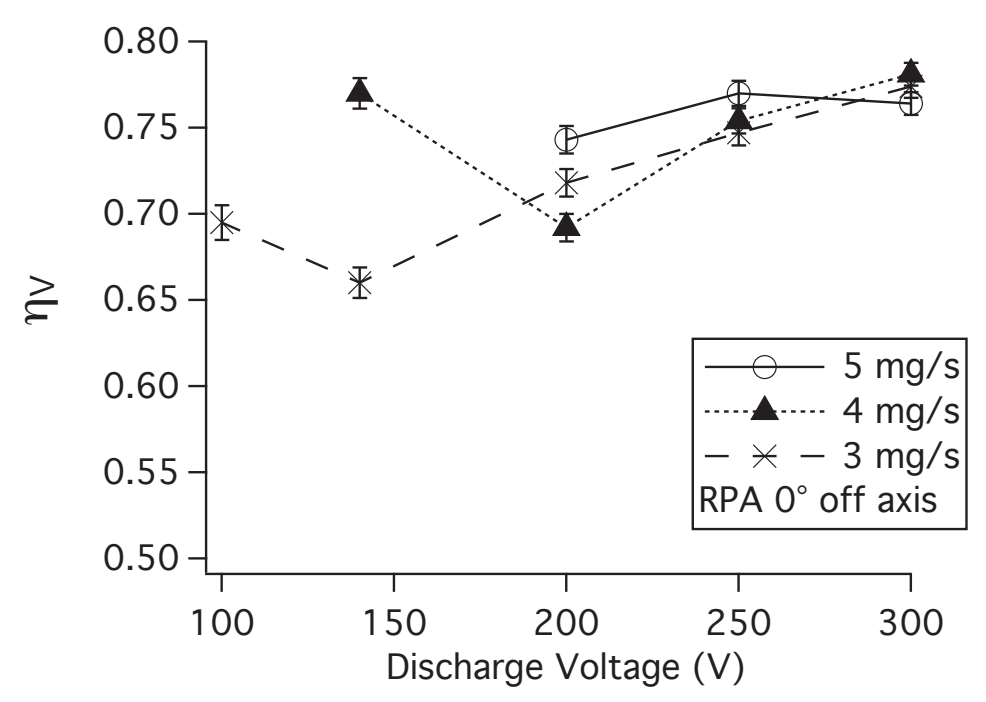

Figure 3.8: $\bar{\epsilon} / e V_{d}$ as calculated from RPA data $0^{\circ}$ off axis and 550 $\mathrm{mm}$ downstream

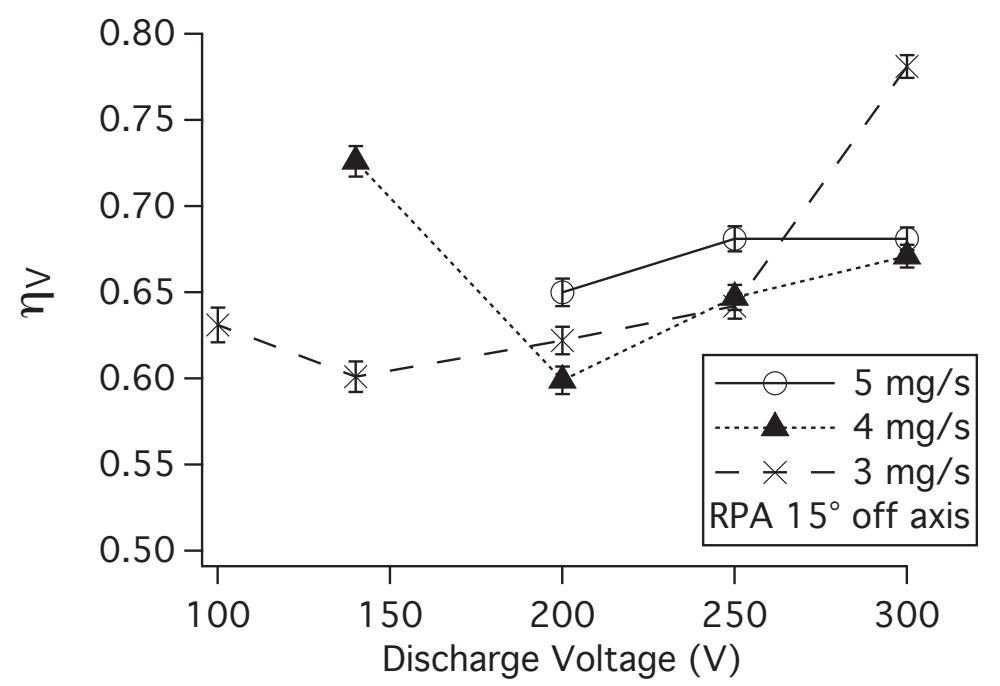

Figure 3.9: $\bar{\epsilon} / e V_{d}$ as calculated from RPA data $15^{\circ}$ off axis and 550 $\mathrm{mm}$ downstream 


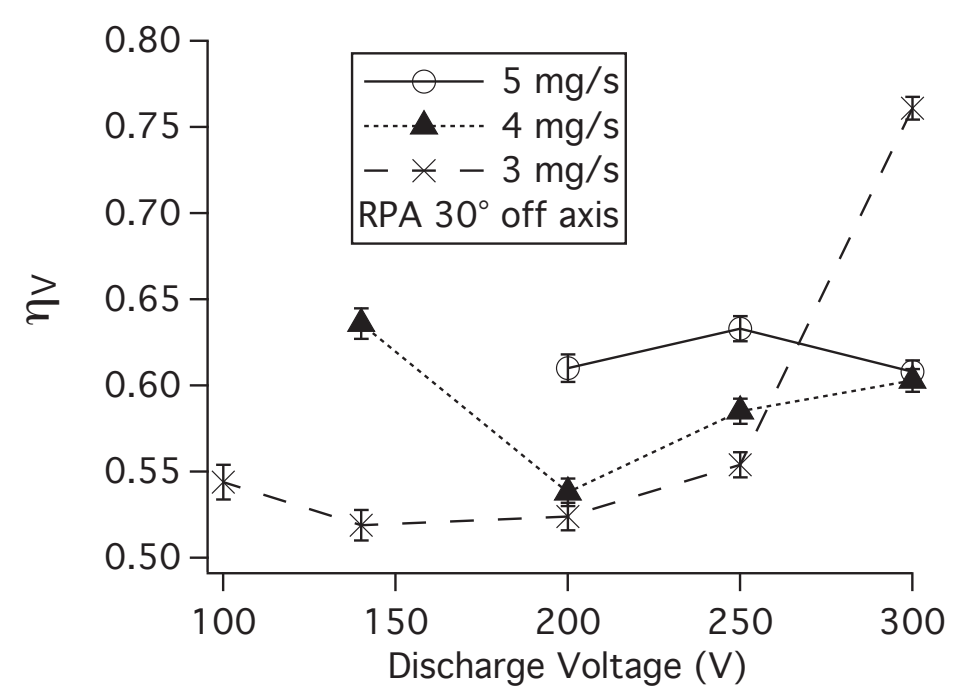

Figure 3.10: $\bar{\epsilon} / e V_{d}$ as calculated from RPA data $30^{\circ}$ off axis and 550 mm downstream

be positioned directly over the apex of the beam profile. If the probe is positioned off apex center, it will result in a sweep that reports an artificially low integrated beam current. The error can be estimated by assuming a maximum vertical probe misalignment of $d x=3 \mathrm{~cm}$ yielding a maximum error in the calculation of $\eta_{B}$ of $3.5 \%$. Additionally, there is an unaccounted error due to the hemispherical integration of charge exchange ions at high angles common to Faraday probe results $[10]$.

\subsubsection{Current Efficiency}

Current efficiency, $\eta_{c}$, is seen in Figure 3.12 where $I_{d}$ is measured directly from the discharge supply. The beam current, $I_{i}$, is obtained from an integration of $j(\theta)$ as measured by the Faraday probe. Current in the beam outside the probe's maximum off-axis angle of $53^{\circ}$ was not collected, and therefore, $I_{i}$ is a lower bound. By using a linear extrapolation of the probe sweeps from $53^{\circ}$ to $90^{\circ}$ (assuming $0 \mathrm{~A} / \mathrm{m}^{2}$ at $90^{\circ}$ ) the amount of current that is omitted from the integration was estimated. This technique indicates the $\eta_{c}$ calculations have a maximum underestimation error of $12 \%$ of the stated value due to the uncollected portions of the beam. Previous studies have shown, however, that the beam dies off much faster than linearly, and therefore, this error is a conservative estimate. The efficiency decays linearly from $300 \mathrm{~V}-160 \mathrm{~V}$ and then begins to decline more rapidly. 


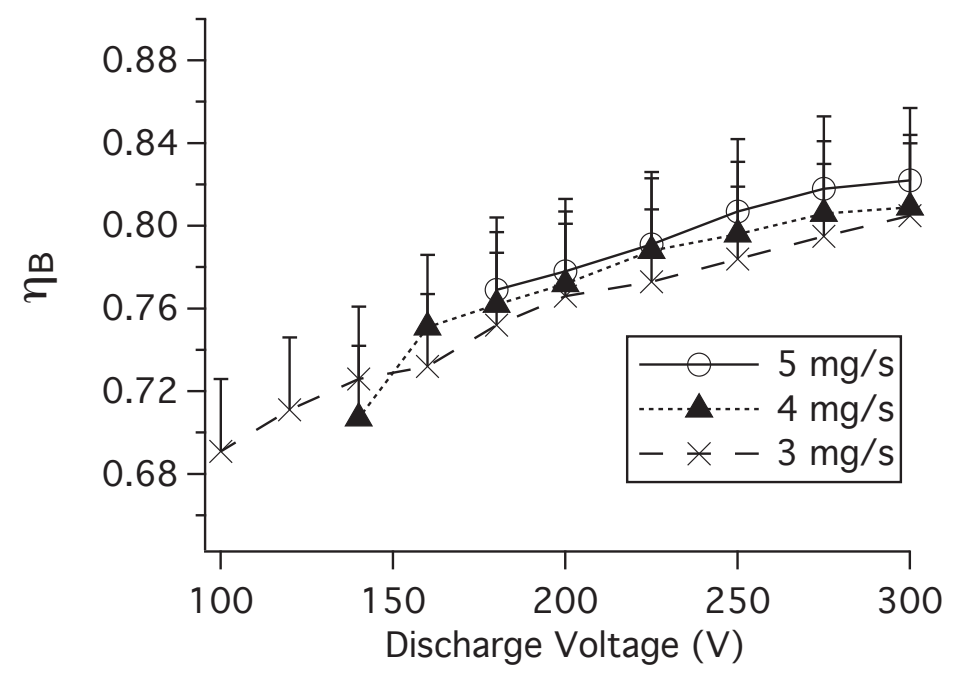

Figure 3.11: Beam divergence efficiency as calculated by Eq. 3.3 from Faraday probe sweeps $250 \mathrm{~mm}$ downstream

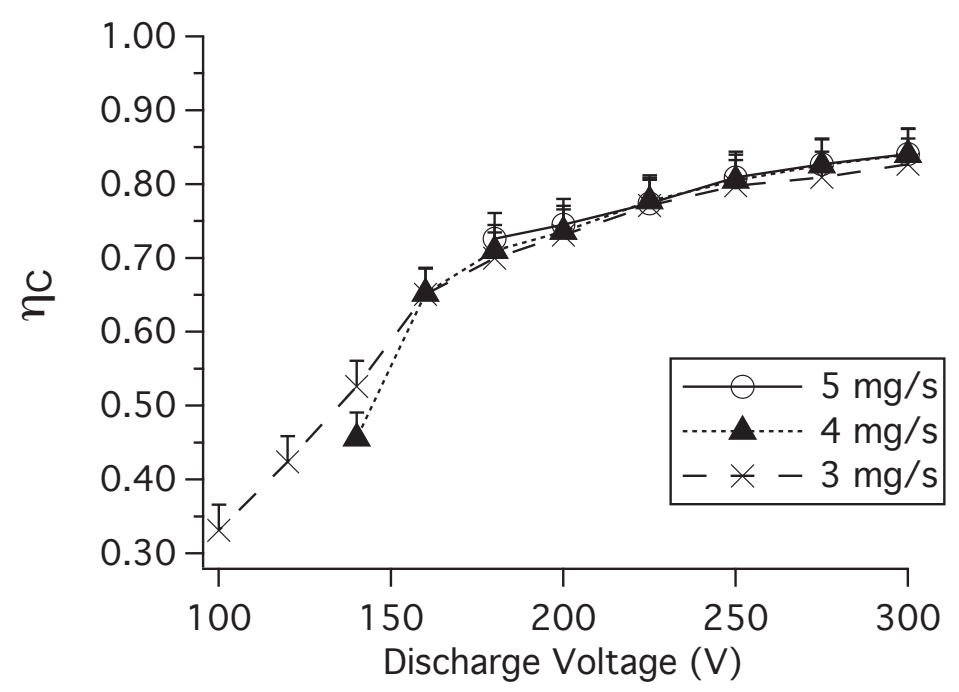

Figure 3.12: Current efficiency as a function of discharge voltage 


\subsection{Summary}

Table 3.4 is summary of all of the findings in this experiment. Plasma-to-cathode values are displayed as determined by the emissive probe at $0.55 \mathrm{~m}$ downstream $0^{\circ}$ off axis. The probe studies measured three of the four loss mechanisms comprising thrust efficiency. The efficiency velocity distribution function, $\eta_{v d f}$, was not obtained, and therefore, ideally, the 'probe efficiency' is an upper bound on the thrust efficiency. Probe efficiency is defined as

$$
\eta_{\text {probe }}=\eta_{v} \eta_{c} \eta_{B}=\frac{\eta_{T}}{\eta_{v d f}}
$$

Although there was a monotonic drop in beam divergence as the discharge voltages were decreased, the change amounted to a small fraction of the decreasing thrust efficiency. The more interesting results are in the current efficiency and voltage utilization efficiencies. The current efficiency suffered greatly at low voltages and was the dominate loss mechanism which is inline with what Brown found in his work [?]. This is not altogether surprising in that the magnetic field was necessarily tuned to a very low level to obtain maximum thrust efficiency. The end effect is that electron trapping force at the exit plane is reduced and many more electrons than necessary are collected at the anode. Thus, the poor current efficiency is easy to explain. Why the maximum thrust efficiency occurred at such a low magnetic field, however, value is not. The coupled decrease in magnetic field strength with decreasing discharge voltage to maintain maximum thrust efficiency has been well observed in the community, but has never been fully explained. In part, this observation fueled the design of the off-peak efficiency probe studies of the following chapter (test 2).

The voltage utilization efficiency, VUE, also exhibited peculiar behavior. The decrease in magnetic field strength did not overly seem to be detrimental to the VUE. In addition, two of the lowest magnetic field values tested showed an increase in voltage utilization efficiency over their higher/nominal magnetic field values. To date, this phenomenon had not been recorded before. 


\begin{tabular}{|c|c|c|c|c|c|c|c|c|c|c|}
\hline $7^{\circ}$ & $c^{5}$ & & 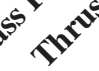 & $c^{2}$ & $\mathbf{p}^{0^{s}}$ & $n$ & $N$ & r & n & 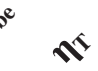 \\
\hline 300 & 2.85 & 3 & 65.63 & -27.00 & 46.10 & 0.78 & 0.80 & 0.78 & 0.49 & 0.50 \\
\hline 250 & 2.84 & 3 & 57.12 & -26.30 & 41.85 & 0.74 & 0.78 & 0.78 & 0.45 & 0.46 \\
\hline 200 & 2.89 & 3 & 45.62 & -22.50 & 35.07 & 0.72 & 0.77 & 0.77 & 0.43 & 0.36 \\
\hline 140 & 3.59 & 3 & 31.96 & -13.90 & 18.53 & 0.70 & 0.73 & 0.62 & 0.31 & 0.20 \\
\hline 100 & 4.81 & 3 & 26.77 & -9.70 & 12.61 & 0.72 & 0.69 & 0.46 & 0.23 & 0.15 \\
\hline 300 & 3.92 & 4 & 73.60 & -28.10 & 49.88 & 0.79 & 0.81 & 0.76 & 0.48 & 0.58 \\
\hline 250 & 3.90 & 4 & 63.82 & -25.90 & 44.29 & 0.76 & 0.80 & 0.76 & 0.46 & 0.52 \\
\hline 200 & 3.99 & 4 & 51.40 & -24.60 & 37.65 & 0.71 & 0.77 & 0.74 & 0.41 & 0.41 \\
\hline 140 & 6.26 & 4 & 43.44 & -11.10 & 17.88 & 0.80 & 0.71 & 0.47 & 0.27 & 0.27 \\
\hline 300 & 4.96 & 5 & 72.63 & -28.70 & 54.32 & 0.80 & 0.82 & 0.75 & 0.49 & 0.59 \\
\hline 250 & 5.01 & 5 & 64.71 & -24.40 & 45.39 & 0.79 & 0.81 & 0.74 & 0.47 & 0.56 \\
\hline 200 & 5.15 & 5 & 53.21 & -23.90 & 41.28 & 0.74 & 0.78 & 0.72 & 0.42 & 0.46 \\
\hline
\end{tabular}

Table 3.4: Comprehensive summary of all $\eta$ values 


\section{Bibliography}

[1] King, D., Tilley, D., Aadland, R., Nottingham, K., Smith, R., Roberts, C., Hruby, V., Pote, B., and Monheiser, J., "Development of the BPT family of U.S.-designed Hall current thrusters for commercial LEO and GEO applications," 34th AIAA/ASME/SAE/ASEE Joint Propulsion Conference and Exhibit, Vol. AIAA-1998-3338, Cleveland, Ohio, July 13-15 1998.

[2] Kieckhafer, A., The Effect of Segmented Anodes on the Performance and Plume of a Hall Thruster, Ph.D. thesis, Michigan Technological University, 2007.

[3] Sommerville, J. D., Hall-Effect Thruster-Cathode Coupling: The Effect of Cathode Position and Magnetic Field Topology, Ph.D. thesis, Michigan Technological University, 2009.

[4] Sommerville, J. D. and King, L. B., "Ion-Collision Emission Excitation Cross Sections for Xenon Electric Thruster Plasmas," Journal of Propulsion and Power, Vol. 24, No. 4, July-August 2008, pp. 880-888.

[5] Kieckhafer, A. W., Massey, D. R., and King, L. B., "Performance and Active Thermal Control of 2-kW Hall Thruster with Segmented Electrodes," Journal of Propulsion and Power, Vol. 23, No. 4, July-August 2007, pp. 821.

[6] Ross, J. L. and King, L. B., "Energy Efficiency in Low Voltage Hall Thrusters," 43rd AIAA/ASME/SAE/ASEE Joint Propulsion Conference and Exhibit, Vol. AIAA-2007-5179, Cinncinatti, OH, July 8-11 2007.

[7] Ross, J. L. and King, L. B., "Efficiency Analysis of a Low Discharge Voltage Hall Thruster," 44th AIAA/ASME/SAE/ASEE Joint Propulsion Conference and Exhibit, Hartford, CT, July 20-23 2008.

[8] Haag, T. W., "Design of a thrust stand for high power electric propulsion devices," 25th AIAA/ASME/SAE/ASEE Joint Propulsion Conference 83 Exhibit, Vol. AIAA-1989-2829, Monterey, CA, July 10-13 1989. 
[9] King, L. B., Transport-Property and Mass Spectral Measurements in the Plasma Exhaust Plume of a Hall-Effect Space Propulsion System, Ph.D. thesis, University of Michigan, 1998.

[10] Larson, C. W., Brown, D. L., and Hargus, W. A., "Thrust Efficiency, Energy Efficiency, and the Role of the VDF in Hall Thruster Performance Analysis," 43rd AIAA/ASME/SAE/ASEE Joint Propulsion Conference and Exhibit, Vol. AIAA 2007-5270, Cincinnati, Ohio, July 8-11 2007. 


\section{Chapter 4}

\section{Loss Mechanisms as a Function of Magnet Current}

The parametric study presented in the last chapter isolated two loss mechanisms of interest: the voltage utilization efficiency $\eta_{v}$, and the current efficiency, $\eta_{c}$. The points of interest were:

- At $150 \mathrm{~V} V_{d}-4 \mathrm{mg} / \mathrm{s}$ and at $100 \mathrm{~V}-3 \mathrm{mg} / \mathrm{s}$ the voltage utilization efficiency indicated an increase in $\eta_{v}$ for the lowest anode potentials.

- At low discharge voltages, the maximum thrust efficiency did not correspond to the minimum discharge current.

The first point was far from conclusive and simply warranted further investigation. The second point, however, is in conflict with existing data at higher discharge voltages, where it is well known that thrust efficiency peaks when the discharge current is minimized by magnetic tuning [1]. The english translation of the 1989 Russian papers titled "Satsionarnyye plasmennyye dvigateli" stated:

... it was found that for fixed values of $V_{d}$ and $\dot{m}$ there is an optimum value of the magnetic field induction at which the maximum efficiency is obtained. Thus, the value of the discharge current $I_{d}$ reaches a minimum [2].

Since this was the not case for the low-voltage thruster, an experiment was designed to investigate these loss mechanisms as a function of changing magnet current. In a parallel study, Brown also found that minimum current to the anode was not synonymous with maximum efficiency for his $6 \mathrm{~kW}$ low voltage thruster[3]. 


\subsection{Experimental Design}

The setup for this experiment was similar to the first, with both a thrust stand section and external probe section. Originally, this test was performed with xenon as the propellant in 2007. Shortly thereafter, the cost of xenon became cost prohibitive for testing. Therefore, the same experiments, with a refined test matrix, were performed with krypton one year later under the assumption that the referenced xenon data would not be used. Fortunately, the cost of xenon fell, both original propellants were able to be used for the final high-speed probe experiment. In the end, having the two sets of data simply strengthens the conclusions of this experiment. It is regrettable, however, that the test matrices create some discontinuity in their presentation in this chapter. This was resolved for the high-speed probe test.

\subsubsection{Test Matrices}

For this round of experiments, the thrust stand test and the probe study used the same set of operating parameters for a given propellant. However, as previously noted, the matrices differ between propellants (see table 4.1(a) and 4.1(b)). A single flow rate of $3 \mathrm{mg} / \mathrm{s}$ case was chosen for xenon since, in the parametric study, it was the only flow rate to successfully complete the entire 300 - 100 $\mathrm{V}$ test range. Additionally, neither of the two "points of interest" motivating this experiment were flow rate dependent. In a preliminary experiment it was determined that $5 \mathrm{mg} / \mathrm{s}$ resulted in the most stable operation at low voltages for krypton, and therefore was used for all subsequent testing.

\subsubsection{Equipment}

All of the equipment used, including the thruster, tank, probes etc. remain unchanged from the previous test with one exception. Due to an onsite parallel testing on the effects of cathode positioning, the choice was made to universalize the mounting location of the cathode on top of the thruster which positioned the exit orifice slightly further away from the centerline of the Hall thruster [4]. This positioning was maintained for Test 3 for the internal probe studies. In comparison to Test 1 , the effects on thrust efficiency are negligible at high discharge voltages, but negatively effected the performance at low discharge voltages. This can be attested to the separatrix theories of Sommerville's work and should be referenced for more information [4]. As all comparative results and conclusions at the end of this dissertation were drawn from Test 2 and Test 3, this does not affect their validity. 
Table 4.1: Test parameters for xenon and krypton

(a) Xenon test matrix

\begin{tabular}{|c|c|c|}
\hline$V_{d}(\mathrm{~V})$ & $I_{m}(\mathrm{~A})$ & $\dot{m}(\mathrm{mg} / \mathrm{s})$ \\
\hline 200 & 2.50 & 3 \\
200 & 2.25 & 3 \\
200 & 2.00 & 3 \\
200 & 1.75 & 3 \\
200 & 1.50 & 3 \\
200 & 1.25 & 3 \\
200 & 1.00 & 3 \\
200 & 0.75 & 3 \\
200 & 0.50 & 3 \\
200 & 0.25 & 3 \\
\hline 150 & 2.50 & 3 \\
150 & 2.25 & 3 \\
150 & 2.00 & 3 \\
150 & 1.75 & 3 \\
150 & 1.50 & 3 \\
150 & 1.25 & 3 \\
150 & 1.00 & 3 \\
150 & 0.75 & 3 \\
150 & 0.50 & 3 \\
150 & 0.25 & 3 \\
\hline 100 & 2.50 & 3 \\
100 & 2.25 & 3 \\
100 & 2.00 & 3 \\
100 & 1.75 & 3 \\
100 & 1.50 & 3 \\
100 & 1.25 & 3 \\
100 & 1.00 & 3 \\
100 & 0.75 & 3 \\
100 & 0.50 & 3 \\
100 & 0.25 & 3 \\
100 & 0.16 & 3 \\
100 & 0.06 & 3 \\
100 & 0.00 & 3 \\
\hline
\end{tabular}

(b) Krypton test matrix

\begin{tabular}{|c|c|c|}
\hline$V_{d}(\mathrm{~V})$ & $I_{m}(\mathrm{~A})$ & $\dot{m}(\mathrm{mg} / \mathrm{s})$ \\
\hline 300 & 3.00 & 5 \\
\hline 300 & 2.50 & 5 \\
\hline 300 & 2.00 & 5 \\
\hline 300 & 1.50 & 5 \\
\hline 300 & 1.00 & 5 \\
\hline 250 & 3.00 & 5 \\
\hline 250 & 2.50 & 5 \\
\hline 250 & 2.00 & 5 \\
\hline 250 & 1.50 & 5 \\
\hline 250 & 1.00 & 5 \\
\hline 200 & 3.00 & 5 \\
\hline 200 & 2.50 & 5 \\
\hline 200 & 2.00 & 5 \\
\hline 200 & 1.50 & 5 \\
\hline 200 & 1.00 & 5 \\
\hline 150 & 3.00 & 5 \\
\hline 150 & 2.50 & 5 \\
\hline 150 & 2.00 & 5 \\
\hline 150 & 1.50 & 5 \\
\hline 150 & 1.00 & 5 \\
\hline 150 & 0.75 & 5 \\
\hline 150 & 0.50 & 5 \\
\hline 125 & 3.00 & 5 \\
\hline 125 & 2.50 & 5 \\
\hline 125 & 2.00 & 5 \\
\hline 125 & 1.50 & 5 \\
\hline 125 & 1.00 & 5 \\
\hline 125 & 0.75 & 5 \\
\hline 125 & 0.50 & 5 \\
\hline 125 & 0.25 & 5 \\
\hline 100 & 3.00 & 5 \\
\hline 100 & 2.50 & 5 \\
\hline 100 & 2.00 & 5 \\
\hline 100 & 1.50 & 5 \\
\hline 100 & 1.00 & 5 \\
\hline 100 & 0.75 & 5 \\
\hline 100 & 0.50 & 5 \\
\hline 100 & 0.25 & 5 \\
\hline
\end{tabular}




\subsection{Thrust Stand Results}

\subsubsection{Thrust Efficiency}

Thrust efficiency as a function of magnet current can be seen in Figure 4.1 for xenon and in Figure 4.2 for krypton. See chapter 3 for an explanation of the thrust stand and supporting equipment.

For both propellants, at high voltages, a discrete and significant decrease in thruster efficiency occurs as the magnet current is lowered. Although not visible for the $200 \mathrm{~V}$ krypton case, a drop in efficiency occurred at $0.74 \mathrm{~A}$ on the magnets during testing. The thruster did not run stably enough, however, to record the efficiency at magnet currents below $1.0 \mathrm{~A}$ for the $200 \mathrm{~V}$ case. This drop in thrust efficicney was seen for both propellants at all discharge voltages greater than 150 $\mathrm{V}$. This transition was accompanied by a visible change in the plume structure from a columnated beam to a glow discharge. This transition was never seen in the lower voltages, below $200 \mathrm{~V}$, because the plume retained a glow discharge over the entire range of magnet voltages. Figures 4.3 and 4.4 display the visual differences between the two different plume modes. This transition may indicate a change in the plasma formation structure of the Hall thruster, partially motivating the deep probe sweep of the following chapter.

\subsubsection{Discharge Current}

Figures 4.5 and 4.6 show the discharge current as a function of magnet current. With increased magnet current, the discharge current plateaus at the higher voltages. This is a commonly seen phenomena in Hall thrusters. Several of the testing matrix points were omitted where the thruster struggled to remain on.

For xenon, the minimum current to the anode occurred at the maximum magnet current, 3 amps, for all three trials. The maximum thrust efficiency, however, occurred at $1.75 \mathrm{~A}, 1.50 \mathrm{~A}$ and $0.5 \mathrm{~A}$ for $200 \mathrm{~V}, 150 \mathrm{~V}$, and $100 \mathrm{~V} V_{d}$ respectively. The misalignment between minimum current to the anode and maximum thrust efficiency supports findings from chapter 3, and also indicates that maximum current efficiency and maximum thrust efficiency will not coincide.

The magnet current values for maximum efficiency and for minimum current for krypton do not align either, see table 4.2. 


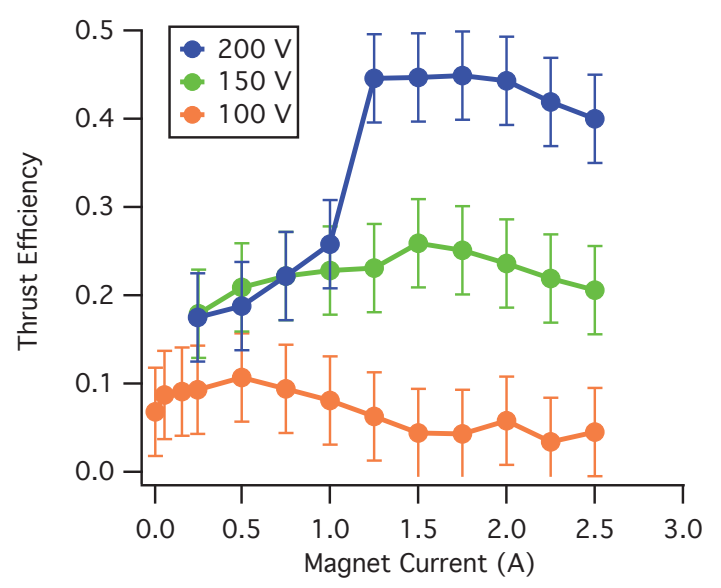

Figure 4.1: Thrust efficiency for xenon

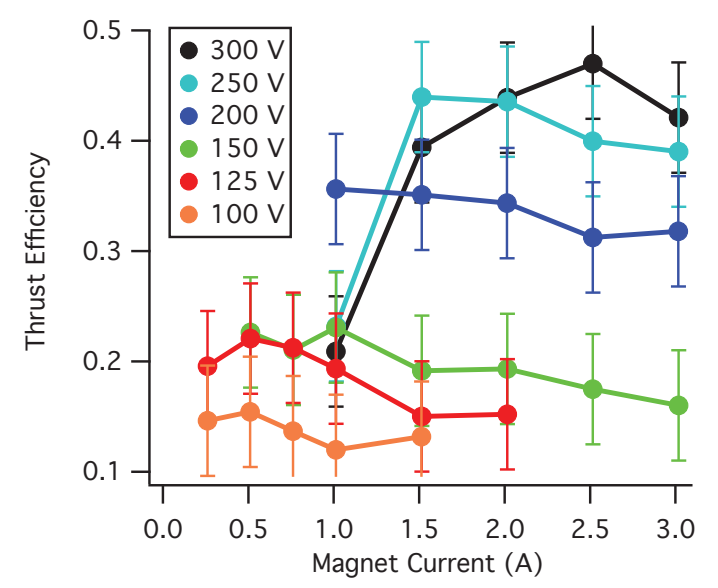

Figure 4.2: Thrust efficiency for krypton

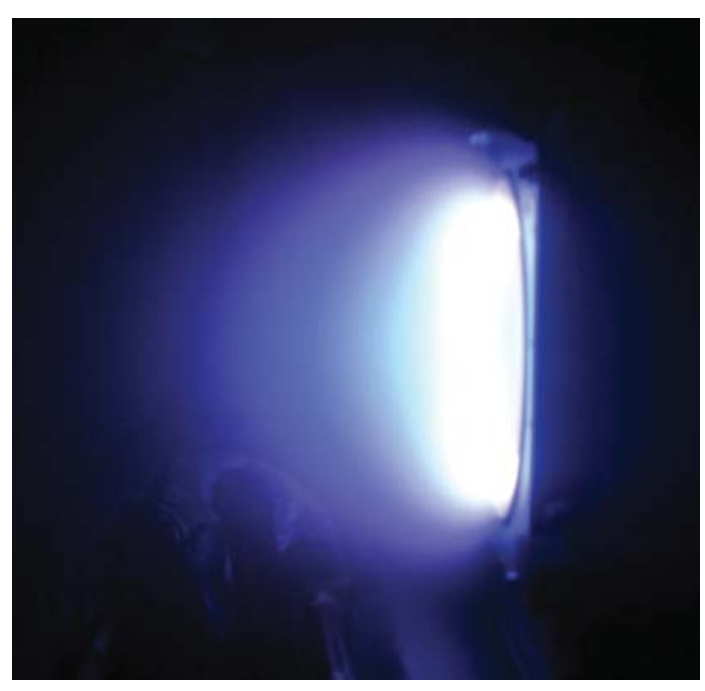

Figure 4.3: Glow Discharge

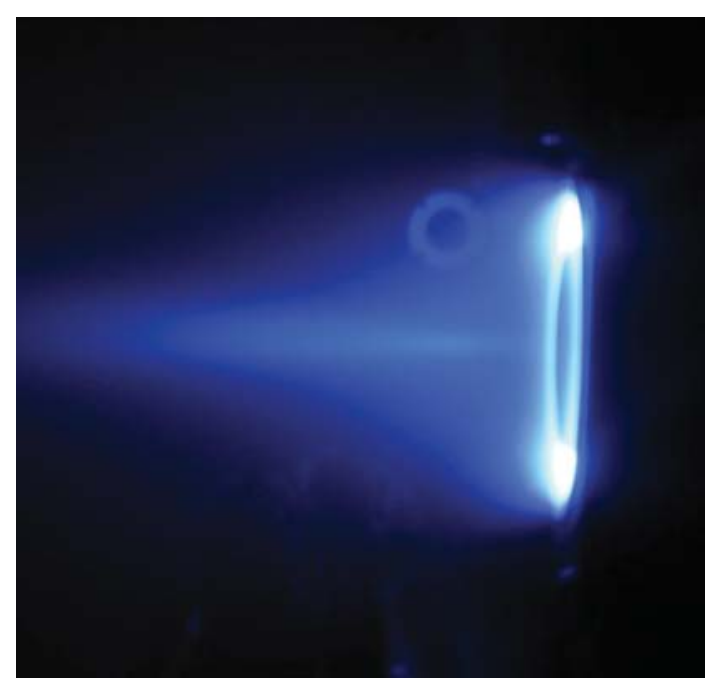

Figure 4.4: Collimated Beam 
Table 4.2: Magnet current values corresponding to maximum efficiency and minimum current to the anode for krypton

\begin{tabular}{|c|c|c|}
\hline$V_{d}(\mathrm{~V})$ & $I_{m}$ for $\max \eta_{T}$ & $I_{m}$ for $\min I_{d}$ \\
\hline 300 & 2.5 & 2.5 \\
250 & 1.5 & 2.0 \\
200 & 1.0 & 3.0 \\
150 & 1.0 & 3.0 \\
125 & 0.5 & 3.0 \\
100 & 0.5 & 1.5 \\
\hline
\end{tabular}

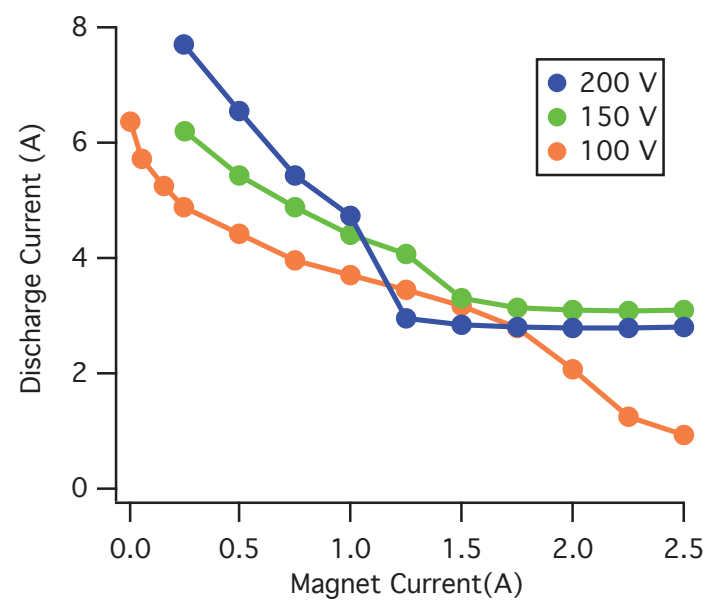

Figure 4.5: Discharge current for xenon

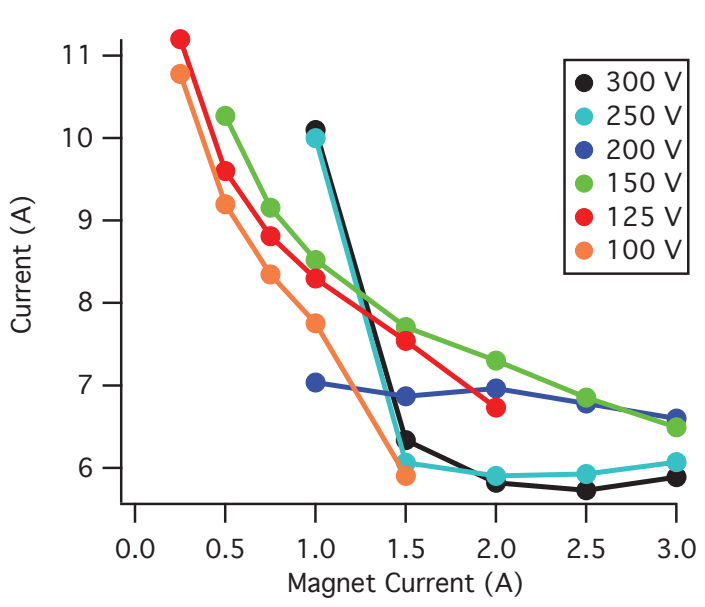

Figure 4.6: Discharge current for krypton 


\subsubsection{Thrust-to-Power}

Thrust-to-power ratios were recorded and displayed as a function of magnet current in Figures 4.7 and 4.8. For both propellants the greatest magnet current resulted in the best thrust-to-power ratio for the $100 \mathrm{~V}$ cases. Interestingly, a second maxima occurred near the peak thrust efficiency magnet current level. Thrust efficiency is quadratically dependent on the exit velocity, whereas the thrust-topower is only linearly dependent on it,

$$
\begin{gathered}
\eta_{T}=\frac{T^{2}}{2 \dot{m} P_{s}}=\frac{\dot{m} U_{e}^{2}}{2 P_{s}} \\
\frac{T}{P_{s}}=\frac{2 \eta_{T}}{U_{e}}=\frac{\dot{m} U_{e}}{P_{s}} .
\end{gathered}
$$

Therefore, to see the thrust-to-power increase at a magnet current value corresponding to a low thrust efficiency indicates the voltage utilization efficiency must be low. This is verified in Figures 4.10 and 4.11.

\subsection{Probe Study}

\subsubsection{Current Efficiency}

Faraday data, as recorded by the procedure outlined in chapter 3, yields beam divergence data and beam current data. The beam current is calculated by integrating the total current recorded by the Faraday probe around a hemisphere to obtain the current efficiency. Due to a computer failure, however, the faraday data for much of the xenon portion of the test was lost. Due to the direction the research was set to take after this set of experiments, it was decided not to go back and retake data. Thus, only the Krypton current efficiency data is presented here.

Current efficiency is plotted in Figure 4.9. In the highest voltage cases, a dip in current efficiency is seen at the greatest magnet currents. In the thrust-topower and thrust efficiency graphs, a similar dip efficiency (within the error bars) is seen for all discharge voltages greater than $150 \mathrm{~V}$ for both propellants. Barring the improbable possibility that the increase in magnetic field strength resulted in an increase in back streaming electrons, this indicates the magnet field strength became too strong to allow the propellant to becoming fully ionized. In other words, the thruster began to eject an increasing fraction of neutrals.

At the lowest of discharge potentials, the current efficiency increases with decreasing magnet current. Since the discharge current increases rapidly as can 


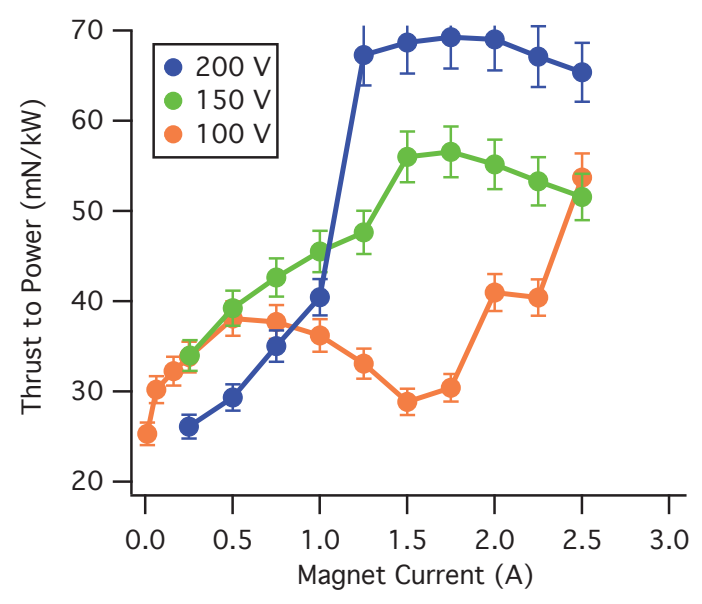

Figure 4.7: Thrust to power results for xenon in $\mathrm{mN} / \mathrm{kW}$

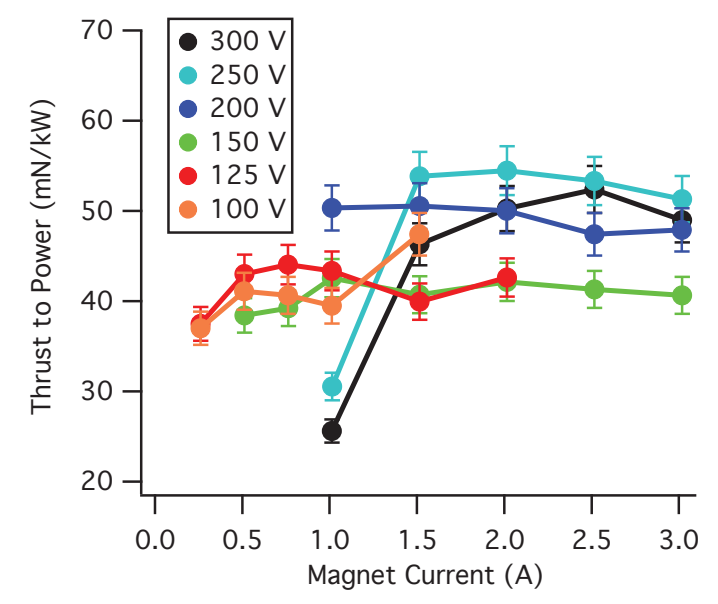

Figure 4.8: Thrust to power results for krypton in $\mathrm{mN} / \mathrm{kW}$

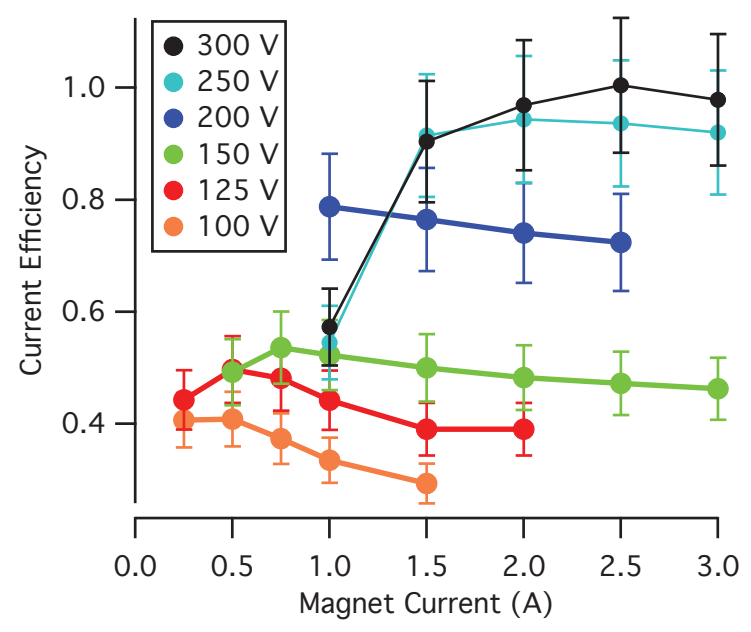

Figure 4.9: Current efficiency for krypton 
be seen in figure 4.6 it can be noted that this increase in current efficiency is due to a drastically increased ionization fraction. At low discharge voltages, the electrons are not energized sufficiently to overcome the nominal magnetic field strength to sustain full ionization of the propellant. This, in part, is why it has historically been empirically shown that decreasing the discharge voltage requires a decrease in the magnetic field strength to increase thrust efficiency.

\subsubsection{Voltage Utilization Efficiency}

The voltage utilization efficiencies (VUE) as recorded by the RPA probe are shown in Figures 4.10 and 4.11. The most important piece information here is that the voltage utilization efficiency is in fact increasing with decreasing magnet current, which is an effect that has been rarely, if ever, seen in published data. The hypothesis here is that the reduced magnetic field strength allows for an increase in back streaming of electrons. This is validated by the increase anode current shown previously. The additional influx of electrons in the discharge chamber causes ionization deeper inside the chamber. It is theorized that the ions are being created in regions of higher electrical potential when the magnet current is low. The subsequent test (chapter 5) was designed, in part, to directly validate or refute this hypothesis.

The considerable VUE gain in the $100 \mathrm{~V}$ and $150 \mathrm{~V}$ case that krypton exhibit over xenon is also notable. Conversely, at $200 \mathrm{~V}$, xenon surpassed krypton in VUE. This confirms the earlier claim that the decrease in thrust efficiency at peak thrust-to-power is an indication of low voltage utilization efficiency.

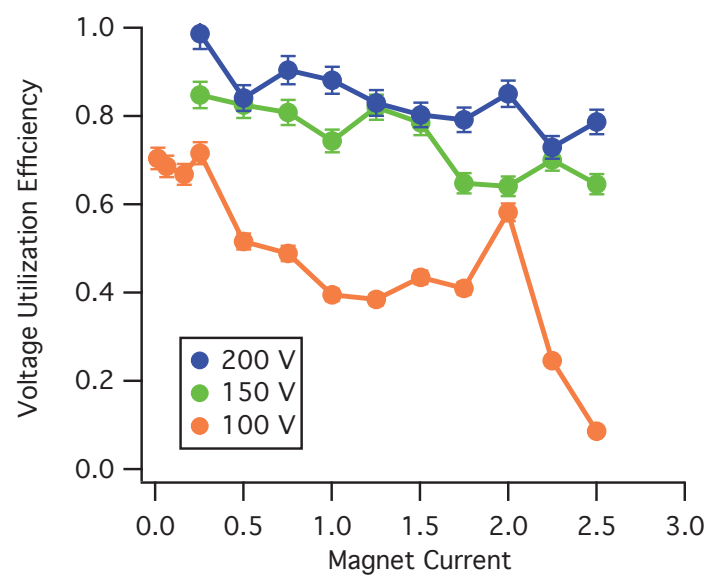

Figure 4.10: Voltage utilization for xenon

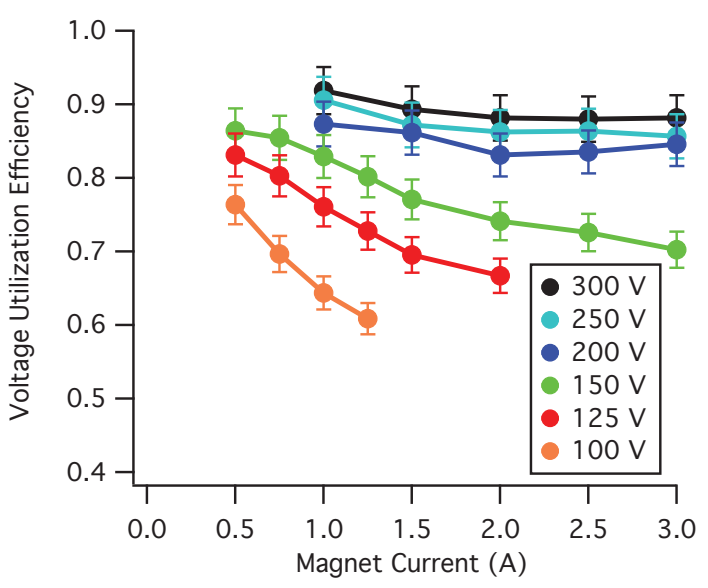

Figure 4.11: Voltage utilization for krypton 
A sample of the raw data for krypton at $125 \mathrm{~V}$ is shown in figures 4.12 4.14. It can be clearly seen that the current to the probe is much greater at the low magnet current levels. This supports the increase in current efficiency shown earlier in figure 4.9. Furthermore, a shift in the IV curve to higher voltages with lower magnet current exists, validating the increase in voltage utilization seen in figure 4.11

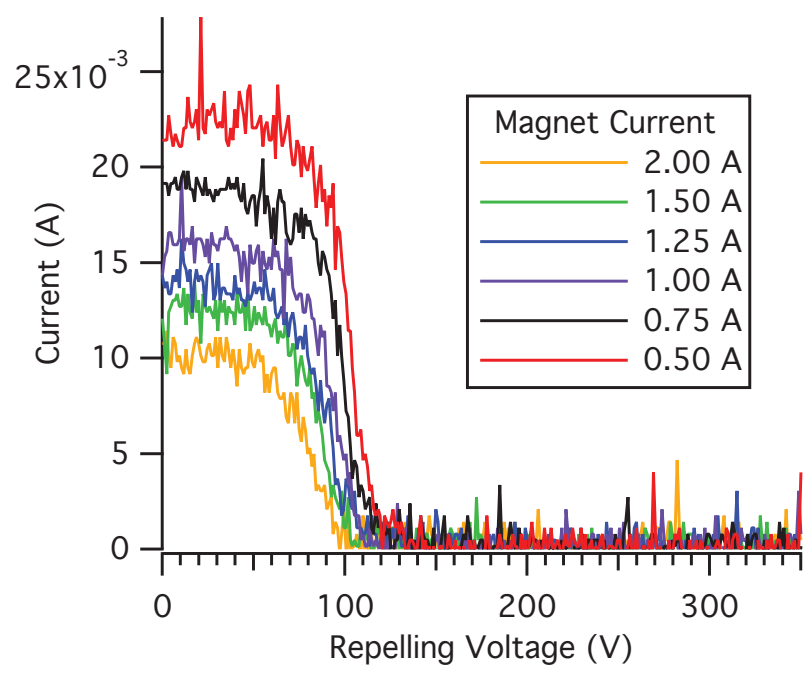

Figure 4.12: Raw RPA IV curves for krypton at $125 \mathrm{~V}$ for various magnet current values. 


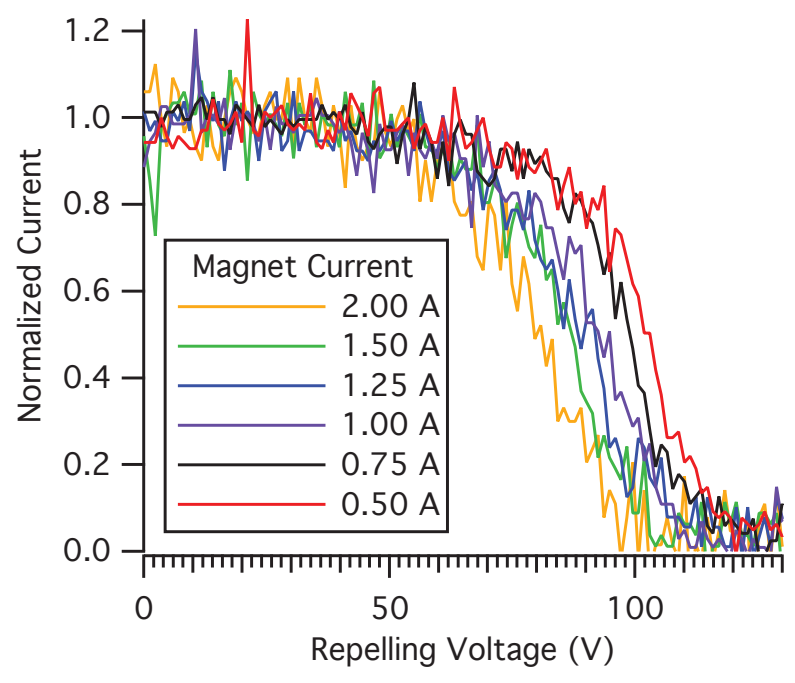

Figure 4.13: Normalized version of figure 4.12 showing RPA IV curves for krypton at $125 \mathrm{~V}$ for various magnet current values.

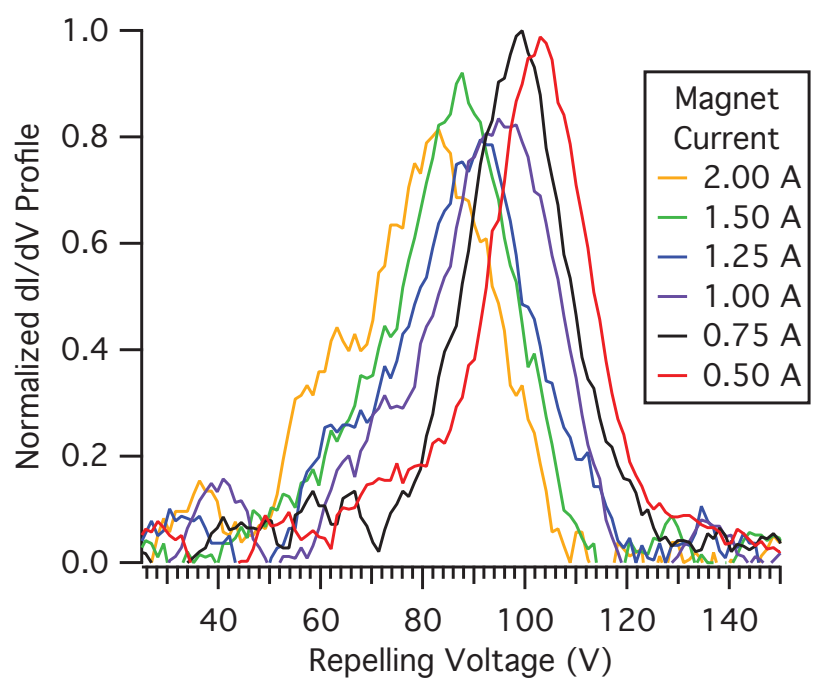

Figure 4.14: Normalized -dI/dV RPA traces for krypton at $125 \mathrm{~V}$ for various magnet current values. 


\section{Bibliography}

[1] Larson, C. W., Brown, D. L., and Hargus, W. A., "Thrust Efficiency, Energy Efficiency, and the Role of the VDF in Hall Thruster Performance Analysis," 43rd AIAA/ASME/SAE/ASEE Joint Propulsion Conference and Exhibit, Vol. AIAA 2007-5270, Cincinnati, Ohio, July 8-11 2007.

[2] Belan, N. V., Kim, V. P., Oranskiy, A. I., and Tikhonov, V. B., "Stationary Plasma Engines - Translation of "Statsionarnyye plasmennyye dvigateli"," UUSR State Committee on Public Education, N. Ye. Zhukovskiy Kharkov Order of Lenin Aviation Institute, 1989, pp. 1-49.

[3] Brown, D. L., Investigation of Low Discharge Voltage Hall Thruster Characteristics and Evaluation of Loss Mechanisms, Ph.D. thesis, University of Michigan, June 2009.

[4] Sommerville, J. D., Hall-Effect Thruster-Cathode Coupling: The Effect of Cathode Position and Magnetic Field Topology, Ph.D. thesis, Michigan Technological University, 2009. 
CHAPTER 4 


\section{Chapter 5}

\section{Design of the High-Speed Probe System}

The High-speed Probe System (HPS) was designed to obtain electron temperature, ion density, and plasma potential for the low-voltage Hall thruster both outside and inside the discharge chamber. From these values the acceleration zone and ionization zone can be inferred for low voltage and low magnet current operation as will be discussed in chapter 7. The system includes the motion table, the AC servo drive system, the double Langmuir probe and accompanying data acquisition system.

\section{$5.1 \quad$ Introduction}

Chapter 4 concluded that voltage utilization efficiency was increasing with decreasing magnet current. This indicated that the plasma acceleration region and/or ionization zone may have moved in axial direction. In order to find out, the electron temperature, floating potential, and plasma potential as a function of the axial direction are needed. Furthermore, since most of the physics of interest happen very close to the anode, measurements will have to include axial locations within the discharge chamber.

In order to take probe measurements inside the discharge chamber, a quick delivery system was necessary to prevent large plasma perturbations and probe ablation and melting. The design and idea of a high-speed probe system was taken from Haas' work in internal Hall thruster measurements [2]. From his work, figure 5.1 depicts the melting time of a probe within a plasma for various peak magnetic fields as a function of axial electric field[1]. The $146 \mathrm{~ms}$ value identified on the graph is for the Fakel SPT-70 Hall thruster. For our Hall thruster, the

BPT-2000, operating at voltages of $300 \mathrm{~V}$ and below, $500 \mathrm{~ms}$ of residence time 
within the discharge chamber based on figure 5.1 is permitted.

In addition to ablation of the probe, plasma perturbation is of concern. Without an exact way to predict the discharge current perturbations our system was going to cause, a goal of maximum discharge chamber residence time of $100 \mathrm{~ms}$ was set. This goal was designed to be in line with a similar system researched [2].

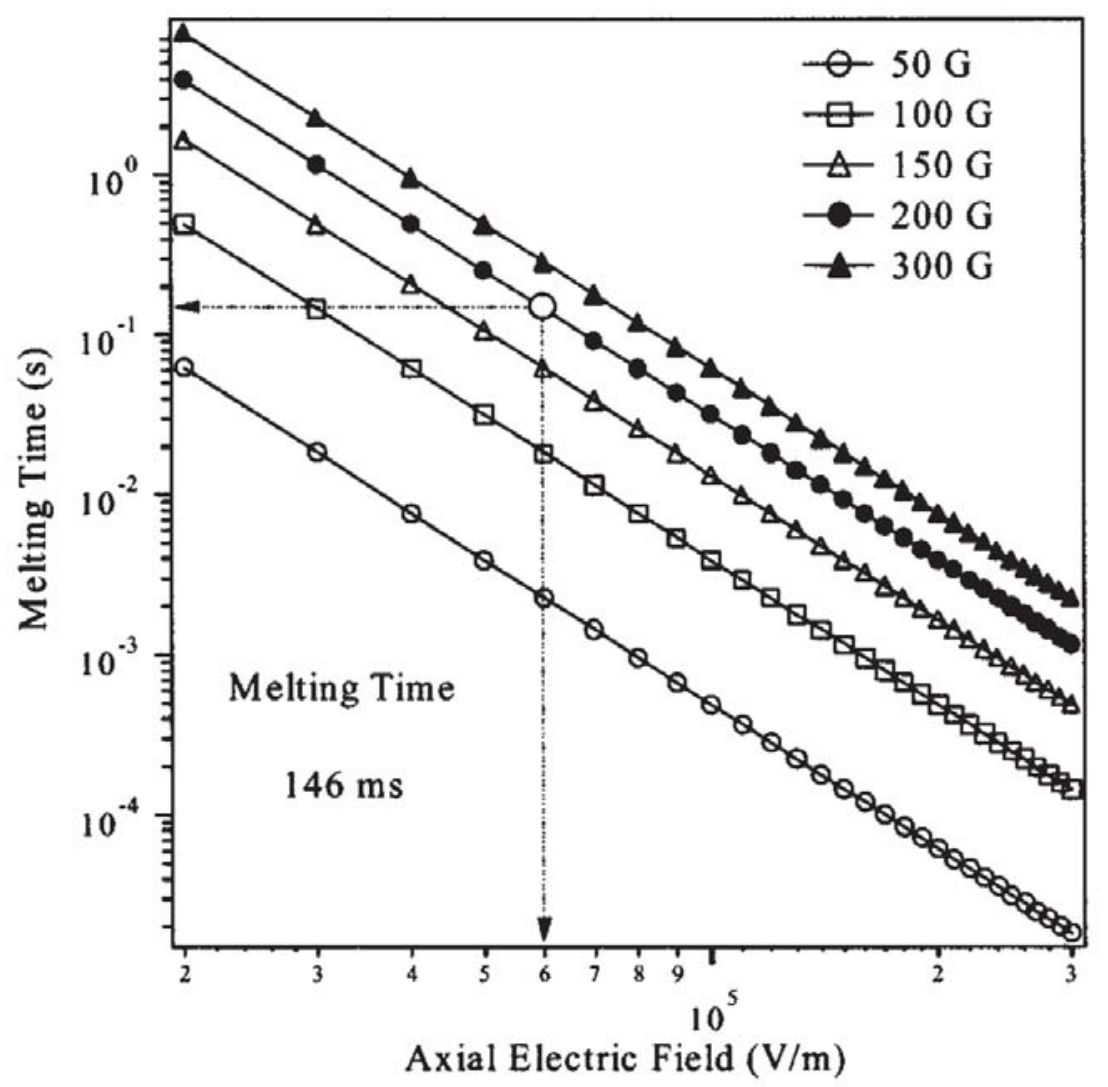

Figure 5.1: Resident melting time of an electrostatic probe in the discharge chamber of a Hall thruster as a function of magnetic and electric field strength: J. M. Haas and A. D. Gallimore. "Development of a high-speed, reciprocating electrostatic probe system for hall thruster interrogation". Review of Scientic Instruments, 71(11), November 2000.

A total of 3 table configurations, 6 servo motors, and countless motor-table couplings were tested before a final product was completed. This chapter outlines the final system in its entirety beginning with the mechanical components and a 
fast motion camera test verifying operation. The design, theory of operation, and analysis of the double Langmuir probe are then discussed, and lastly the in-test current oscillations induced by the probe system are shown.

\subsection{Motion Table}

To limit production time and complication, a goal was set to use as many off-theshelf parts for the system as possible. Since the laboratory in use was already fitted with several motion table systems by the manufacturer Techno-isel, these were utilized for the HPS ${ }^{1}$. Aa the manufacturer's standard DC servo motor offered by the company proved invapable of the acceleration requirements necessary, a Techno-isel Narrow Profile Siled 2 was provided for use.

The motion table, part \# HL31NPMK0690202X, has a $67 \mathrm{~cm}$ functional travel length driven by a a ball screw with a $20 \mathrm{~mm}$ pitch. The factory lubricants were removed and all parts were cleaned with a combination of acetone and isopropyl. A derivative of Braycote, Castrol Optitool 215-2, was used to lubricate the ball screw. Figure 5.2 is the motion table as it was mounted in the tank. The kapton tape is used to hold on the grafoil and is beyond the end of travel.

\subsection{Servo Motor}

The tests used an AC servo system from SureServo. The drive, model SVA-2100, is single phase 220 Volt AC servo with fully configurable onboard memory. The drive was paired with the SVL-202 motor rated for a maximum of $200 \mathrm{~W}$ and rated maxium torque of $1.91 \mathrm{Nm}$. Table 5.1 summarizes the motor specifications.

A custom water cooling heat sink was mounted to the outside of the servo to dissipate heat after a failed qualifying vacuum test determined that, in the absence of convective environment, the servo motor failed from current overload.

Table 5.1: SureServo SVL-200 Servo Motor Specifications

\begin{tabular}{|c|c|c|}
\hline Maximum Torque & 1.91 & $\mathrm{Nm}$ \\
Rated Speed & 3000 & rpm \\
Optical Encoder & 2500 & lines \\
\hline
\end{tabular}

\footnotetext{
${ }^{1}$ http://www.technoautomation.com/
} 


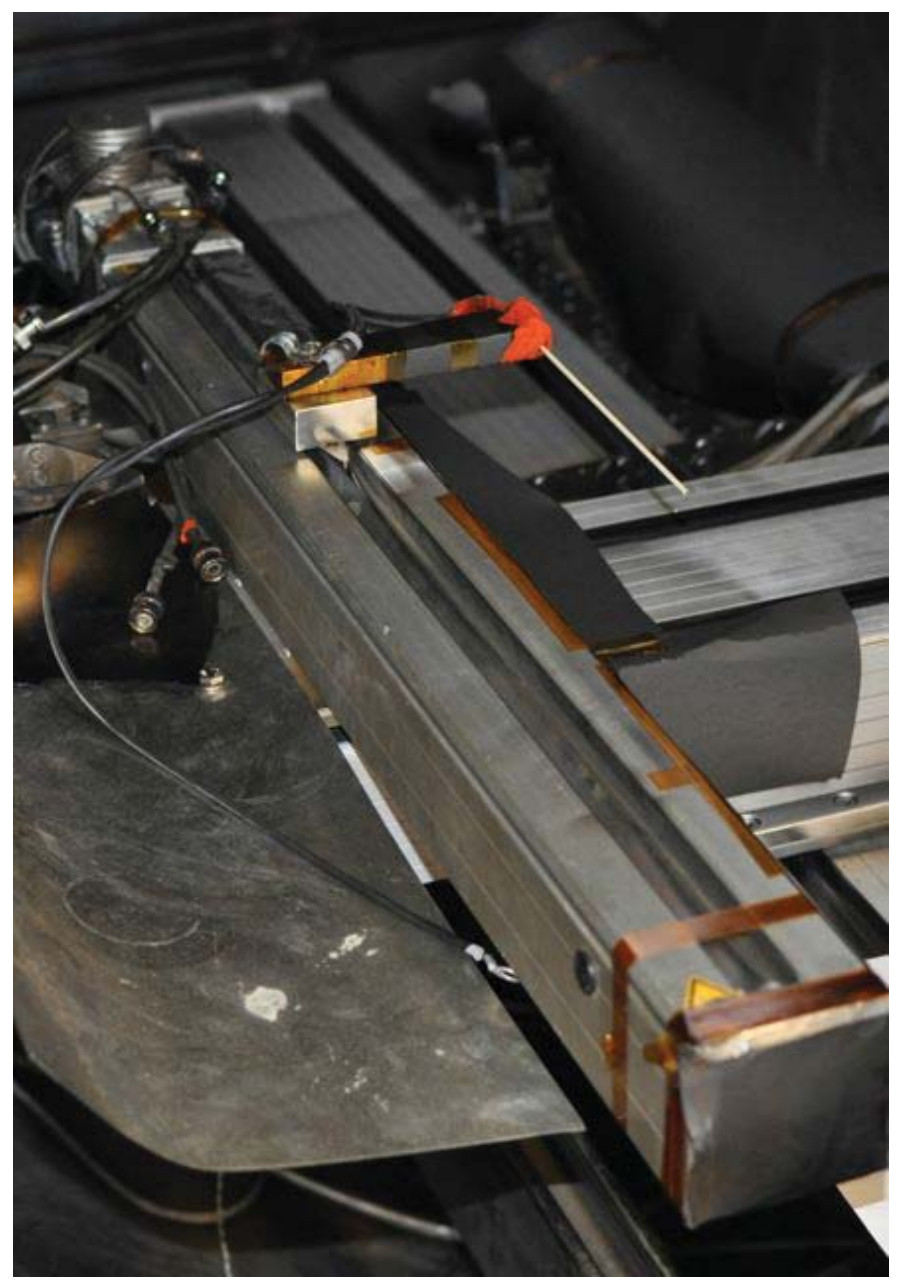

Figure 5.2: Assembled high-speed motion table with mounted double Langmuir probe 


\section{Coupling}

The coupler used to connect the servo motor and motion table was a developed by R\&W Coupling Technology. ${ }^{2}$ A custom housing was fabricated out of aluminum to rigidly mount the motor to the table and allow coupler to unite the shafts. Figure 5.3 shows the servo motor, the aluminum housing and a glimpse at the cylindrical coupler between the table and motor. The two holes drilled in the housing were created to allow adjustment to the coupler.

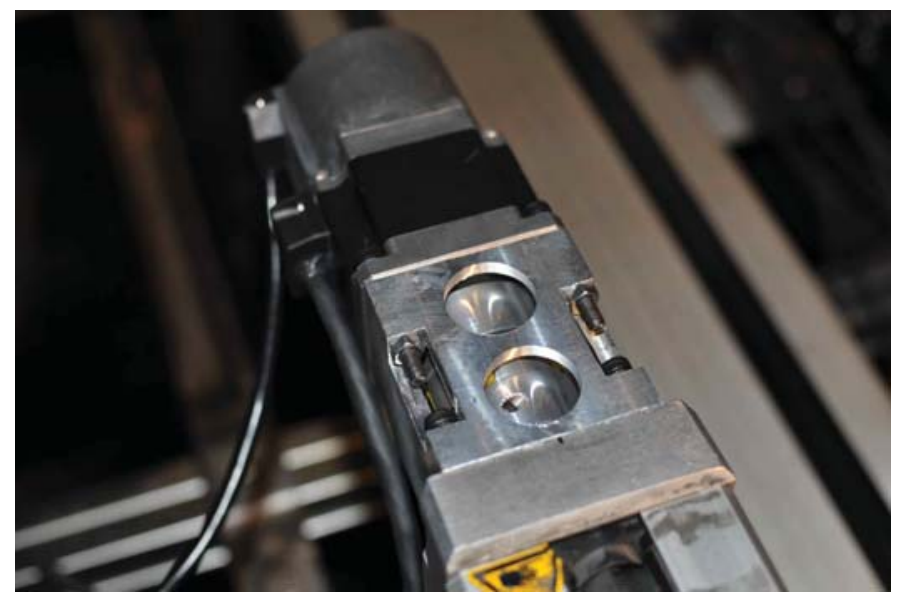

Figure 5.3: Servo motor to motion table coupler

\subsection{Fast Motion Camera Test}

In order to verify accuracy and velocity of the assembled motion system, a fast motion camera test was performed. The camera was positioned at the end of travel and recorded the motion of the table at $1000 \mathrm{fps}$. This test was performed 10 times with power cycles to the probe system in between to guarantee repeatability in the event of a power loss in situ.

In the BPT-2000 the anode face is located $20 \mathrm{~mm}$ behind the exit plane. The probe system was designed to record $18 \mathrm{~mm}$ of this space leaving a $2 \mathrm{~mm}$ buffer between the probe tip and the anode face. Figure 5.4 shows the two exit plane frames and end of travel frame and their time stamps indicating a total residence time of less than $60 \mathrm{~ms} .{ }^{3}$ The test also verified the position accuracy of the probe was consistent to $\pm 0.3 \mathrm{~mm}$.

\footnotetext{
${ }^{2}$ http://www.rw-america.com/

${ }^{3}$ Special thanks to Dr. Jeff Allen and Andrew Shafer of Michigan Technological University
} 


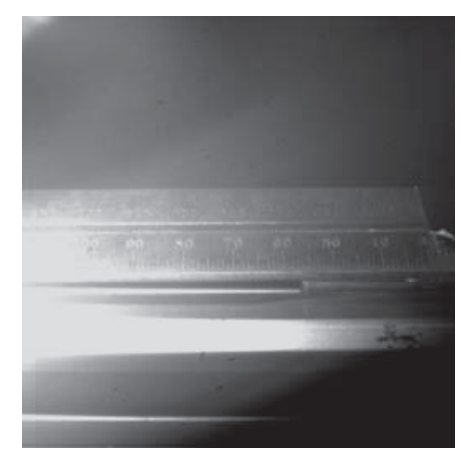

(a) Time $=3 \mathrm{~ms}$

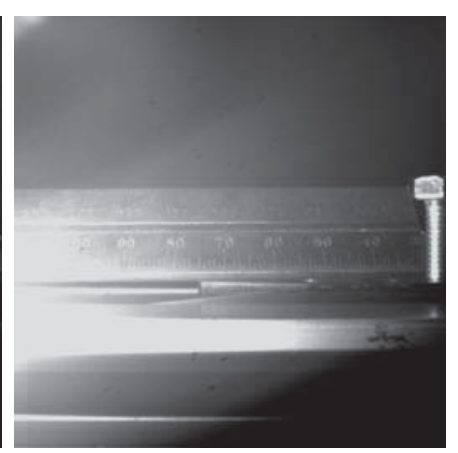

(b) Time $=27 \mathrm{~ms}$

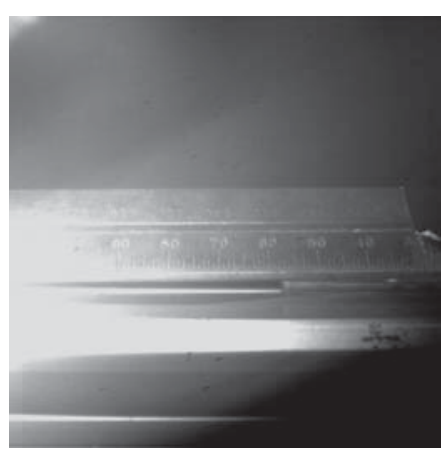

(c) Time $=58 \mathrm{~ms}$

Figure 5.4: Fast motion camera results showing the probe completing the simulated $\pm 18 \mathrm{~mm}$ round trip from the exit plane to the anode and back in less than $60 \mathrm{~ms}$. The motion table is driving the platform from the right to the left in the pictures. The middle picture shows the point of furthest travel where the center screw in the platform is visible. The first last pictures, nearly identical, show the platform entering the frame and leaving the frame respectively. The distance travelled between frames (a) and (b) is $18 \mathrm{~mm}$ and the same between frames (b) and (c).

\subsection{Probe Construction}

In terms of construction, Langmuir probes are among the simplest diagnostic tools to create in electric propulsion laboratories. They consist of a dielectric tube with a protruding refractory metal filament. Most often, alumina or boron nitride is used as dielectric material, however, quartz and graphite have also been used [3]. Used were a $2.54 \mathrm{~mm}$ OD double bore alumina tube with $0.5 \mathrm{~mm}$ OD tungsten filaments that protruded $2 \mathrm{~mm}$. Figure 5.5 shows a close up of the probe tips.

Probe analysis varies depending on the size of the probe. The desired analysis, a Thin-Sheath Limit (TSL), required

$$
\xi=\frac{r_{p}}{\lambda_{d}} \gg 1
$$

the ratio of probe radius to debye length to be much greater than 1 where the debye length is ${ }^{4}$

for the use of their time and equipment for fast cam test.

${ }^{4}$ When $\xi \ll 1$ "orbit motion limit" (OML) theory must be used. 


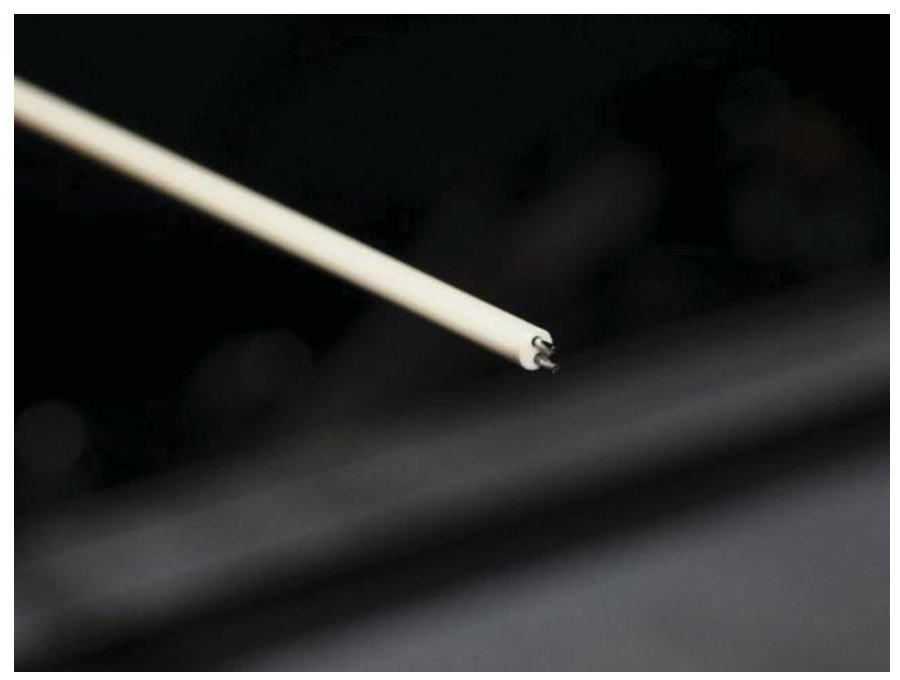

Figure 5.5: Close-up of tungsten probe tips for the double Langmuir probe

$$
\lambda_{d}=\sqrt{\frac{\epsilon_{0} k T_{e}}{e^{2} n_{e}}} .
$$

Assuming a typical electron temperature of $10 \mathrm{eV}$ and a density of $1.5 \times 10^{18}$ $\mathrm{m}^{-3}$ the debye length is $19 \mu \mathrm{m}$. Our probe radius is $250 \mu \mathrm{m}$ which means our $\xi$ is 13 .

\subsection{Analysis}

The presence of the magnetic field in the Hall thruster changes the shape of the sheath surrounding the probe. Since the ions remain unmagnetized, the ion saturation potential is used to calculate the ion density. The current collected by a double probe as a function of voltage is,

$$
I(V)=I_{\text {sat }} \tanh \left(\frac{e V}{2 k T_{e}}\right)
$$

where $I_{\text {sat }}$ is the ion saturation current [3]. Assuming a maxwellian temperature distribution within the plasma, a custom curve fit is constructed,

$$
f(x)=A \tanh (B x+E)+C x+D
$$

where $A, B, C, D$ and $E$ are the fit coefficients to be solved for. The linear portion of the curve fit $C x$ accounts for the growing sheath (effective probe surface area) 
as the voltage is increased. By comparing equations 5.3 and 5.4 the coefficient $A$ is exactly the ion saturation current. Similarly, the electron temperature in electron volts is

$$
T_{e}=\frac{1}{2 B}
$$

The "Bohm Current" or ion saturation current is equal to,

$$
I_{s a t}=n_{i} e A_{s} \sqrt{\frac{k T_{e}}{m_{i}}},
$$

where $n_{i}$ is the ion density (which is the same as the electron density due to quasineutrality), $A_{s}$ is the probe collecting surface area, $m_{i}$ is the ion mass [4]. Taking the ion saturation current from equation 5.4 and the electron temperature from equation 5.5 we can solve for the ion density with equation 5.6. The plasma potential is calculated as

$$
V_{p}=V_{f}+\frac{T_{e V}}{2} \ln \left(\frac{2 m_{i}}{\pi m_{e}}\right)
$$

where $T_{e V}$ is the electron temperature in electron volts [3].

This process was automated using a combination of Matlab and Igor Pro scripts to produce the following three parameters of interest for each double probe trace:

- Electron Temperature

- Electron Density

- Plasma Potential

\subsection{Data Aquisition}

Data acquisition was performed using isolated analog input modules from National Instruments and triggered off the optical encoder clicks of the servo motor. Each encoder click triggered the recording of current, voltage and floating potential of the double probe. Since the servo motor has 2500 line encoder and the ball screw has $20 \mathrm{~mm}$ pitch, 125 data points for each property were recorded per $\mathrm{mm}$. This results in a collection rate of $93.75 \mathrm{kHz}$ for a servo motor speed of $2250 \mathrm{rpm}$.

A floating function generator was used to create a $375 \mathrm{~Hz}$ triangle wave to create the $\pm 40 \mathrm{~V}$ sweep between the probes. The frequency was chosen based on the rotational speed of the servo motor, $2250 \mathrm{rpm}$, to complete one entire sweep 
every $\mathrm{mm}$ at maximum velocity ${ }^{5}$. This gives a spatial resolution of $\pm 0.5 \mathrm{~mm}$ for each I-V curve, however the probe dimensions dictate a $\pm 1 \mathrm{~mm}$ uncertainty. The probe length has a $2 \mathrm{~mm}$ collection length, however, since data is recorded every $\mathrm{mm}$, each data point overlaps $50 \%$ of the region of the previous point and the subsequent point. In this way, a built-in smoothing function is incorporated into the data collection. This was chosen to double the amount of data recorded per sweep and reduce the effects of noise. Figure 5.6 shows the voltage sweep and capacitive current as recorded by the oscilloscope. Due to the fast oscillating voltage, the internal resistance, and length of cable involved in the system, a capacitive current is induced in the lines. Input and output impedances of the cables and measuring equipment were matched to reduce the problem. The capacitive current of the system in vacuum was a maximum of $120 \mu \mathrm{A}$ and was subtracted from the traces in post processing. A diagram of the double probe circuit can be seen in figure 5.7

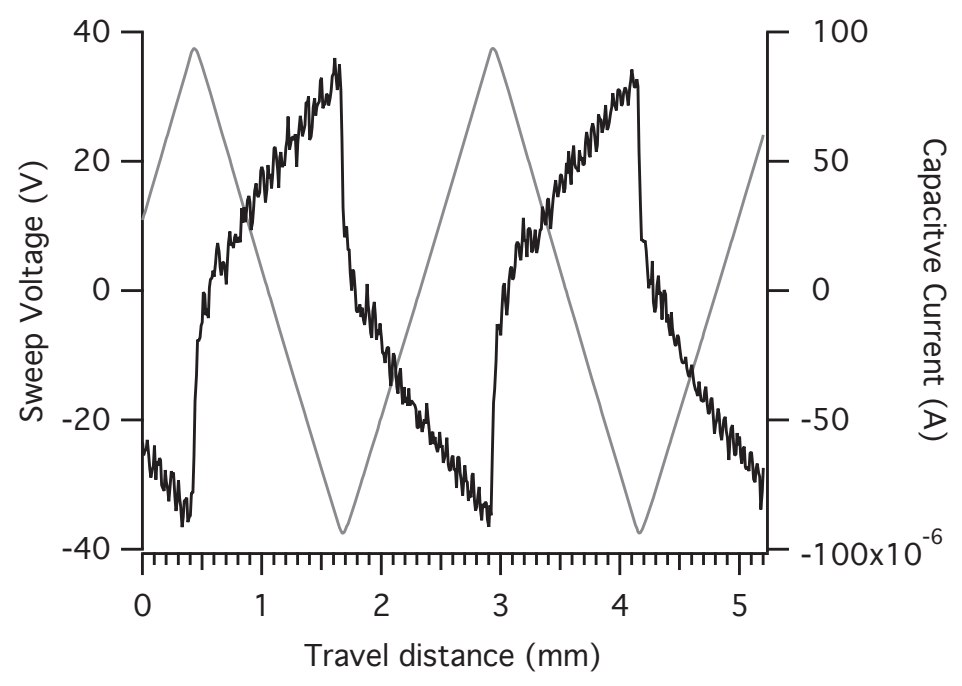

Figure 5.6: Voltage and capacitive current of the sweep circuit. Current is in black and voltage in grey.

\subsection{Perturbations}

Intrusive probe techniques inherently perturb the plasma and thus increase the uncertainty of the measurements. This problem is aggravated in regions of the

\footnotetext{
${ }^{5}$ The rise and decline of the triangle are each considered a complete voltage sweep
} 


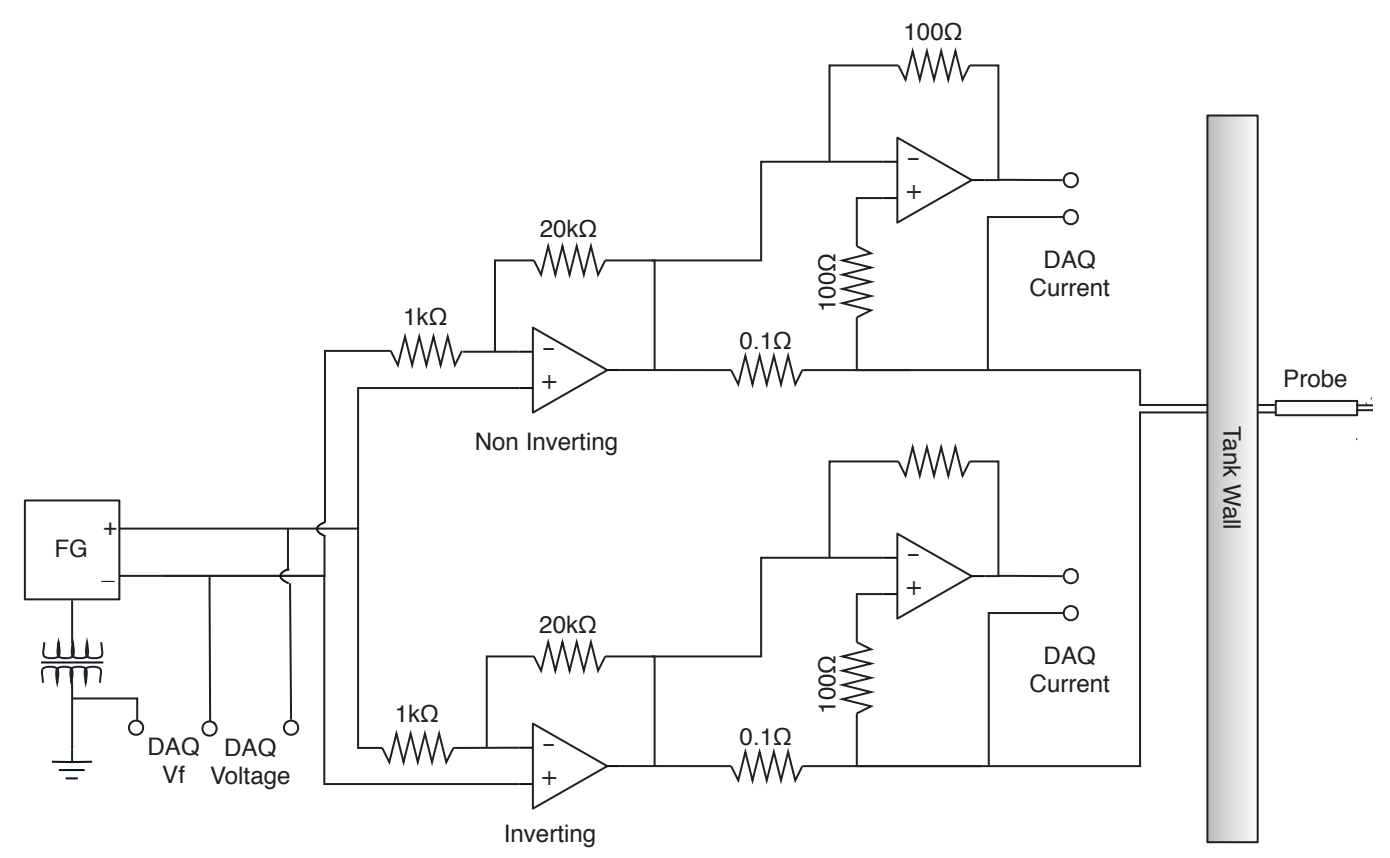

Figure 5.7: Double probe circuit diagram

plasma of where the density and electron energy are very high near the exit plane of the thruster. One measure of the probe's induced disturbance is the fluctuations in the discharge current. Using a high-speed Agilent N2772A $20 \mathrm{MHz}$ differential probe, the discharge current was recorded during normal operation and in the presence of the Langmuir probe at $93.75 \mathrm{kHz}$. The discharge current data was divided into subsets corresponding to $1 \mathrm{~mm}$ of travel of 125 samples each. Each subset of data is then analyzed for a maximum, minimum and average value. Figures 5.8 - 5.12 depict these statistics for the discharge current during the approach, insertion and removal of the Langmuir probe for a variety of test cases. The bottom axis of the figures is the axial location of the probe relative to the exit plane where negative values represent places where the probe was inside the discharge chamber. The dashed lines represent the maximum and minimum current levels and the bold centerline represents the time averaged discharge current. The plots represent the inward sweep (in blue) and a portion of the outward sweep (in red). The region between the two dashed lines in each graph represents the section of data corresponding to the probe being inside the discharge chamber. The inward and outward sweep meet at $-18 \mathrm{~mm}$, the furthest distance traveled by the probe, 


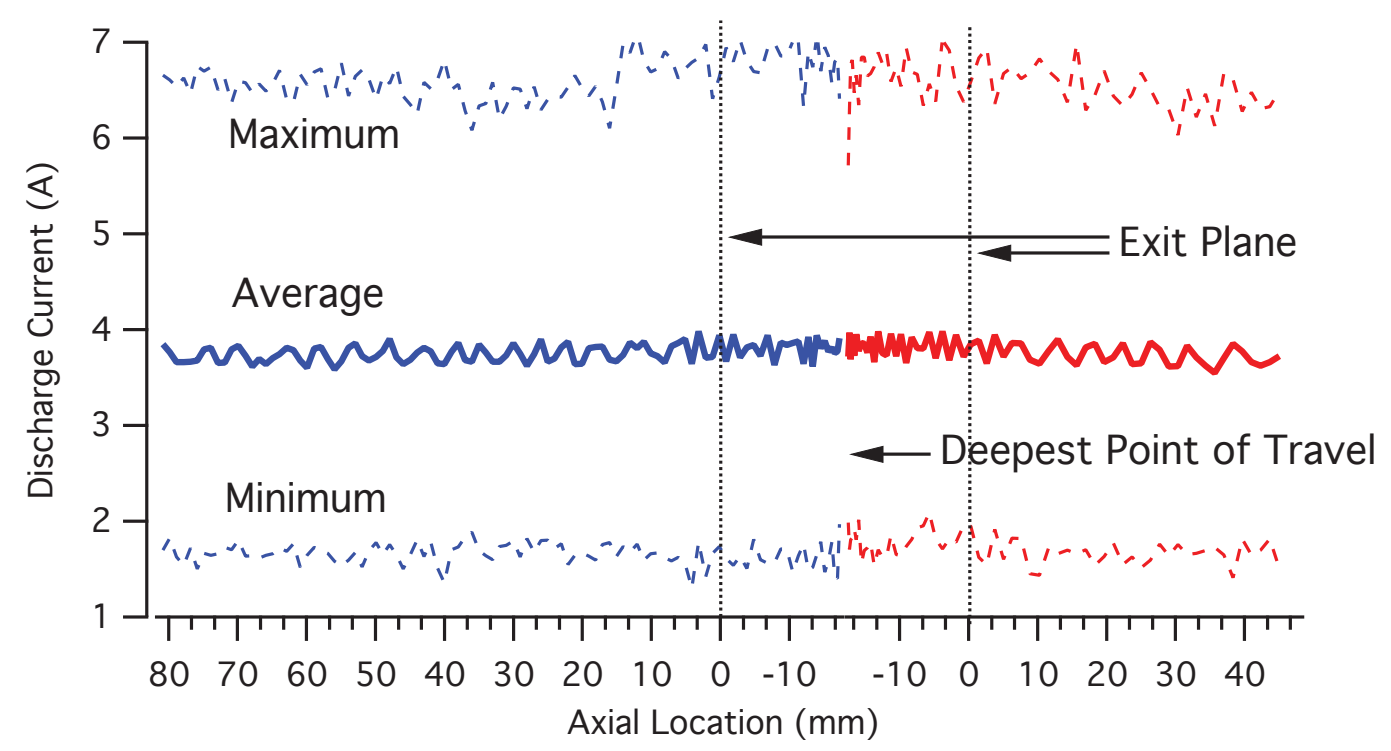

Figure 5.8: Discharge current measurements for xenon at $125 \mathrm{~V}$ as the Langmuir probe is inserted into the discharge chamber. The colored horizontal dashed lines represent the maximum and minimum current where the center bold line represents the average current. The vertical dotted lines represent the exit plane as its passed on the way into the discharge chamber and on the way back out. 


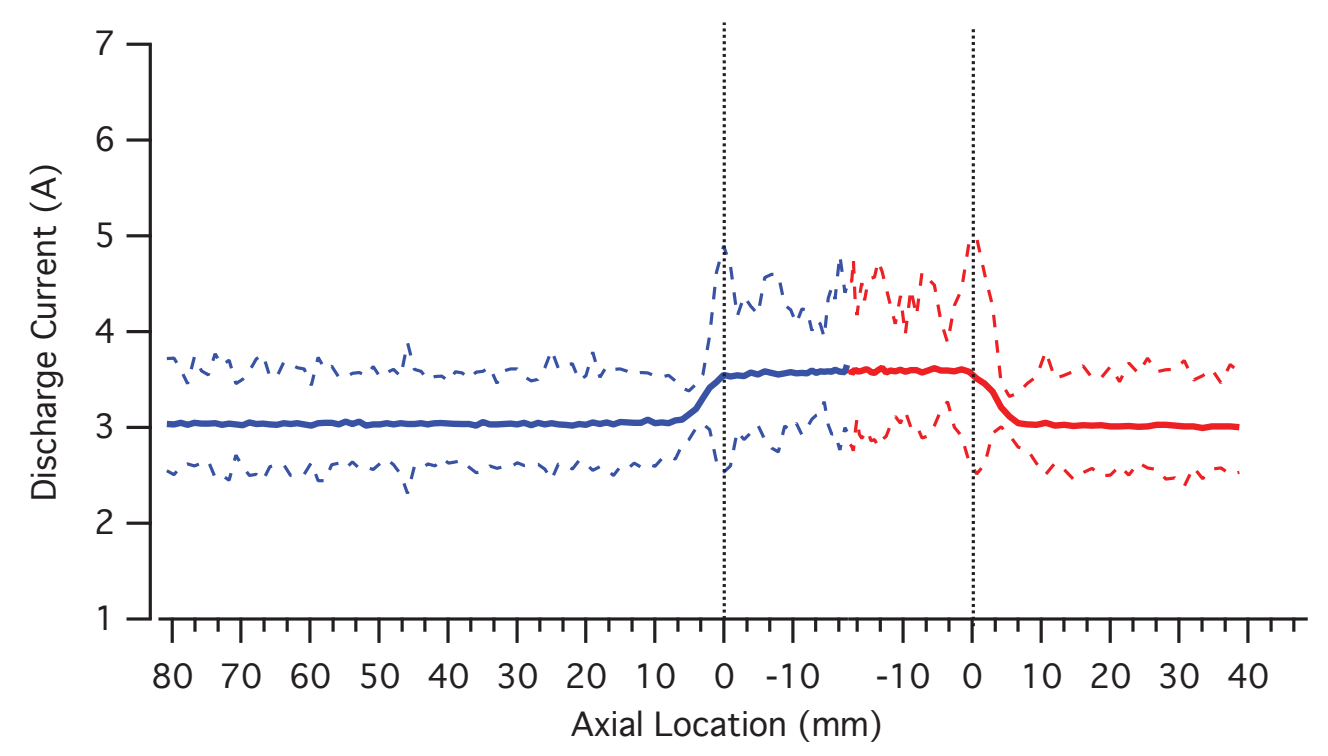

Figure 5.9: Discharge current measurements for xenon at $200 \mathrm{~V}$ as the Langmuir probe is inserted into the discharge chamber. The colored horizontal dashed lines represent the maximum and minimum current where the center bold line represents the average current. The vertical dotted lines represent the exit plane as its passed on the way into the discharge chamber and on the way back out. 


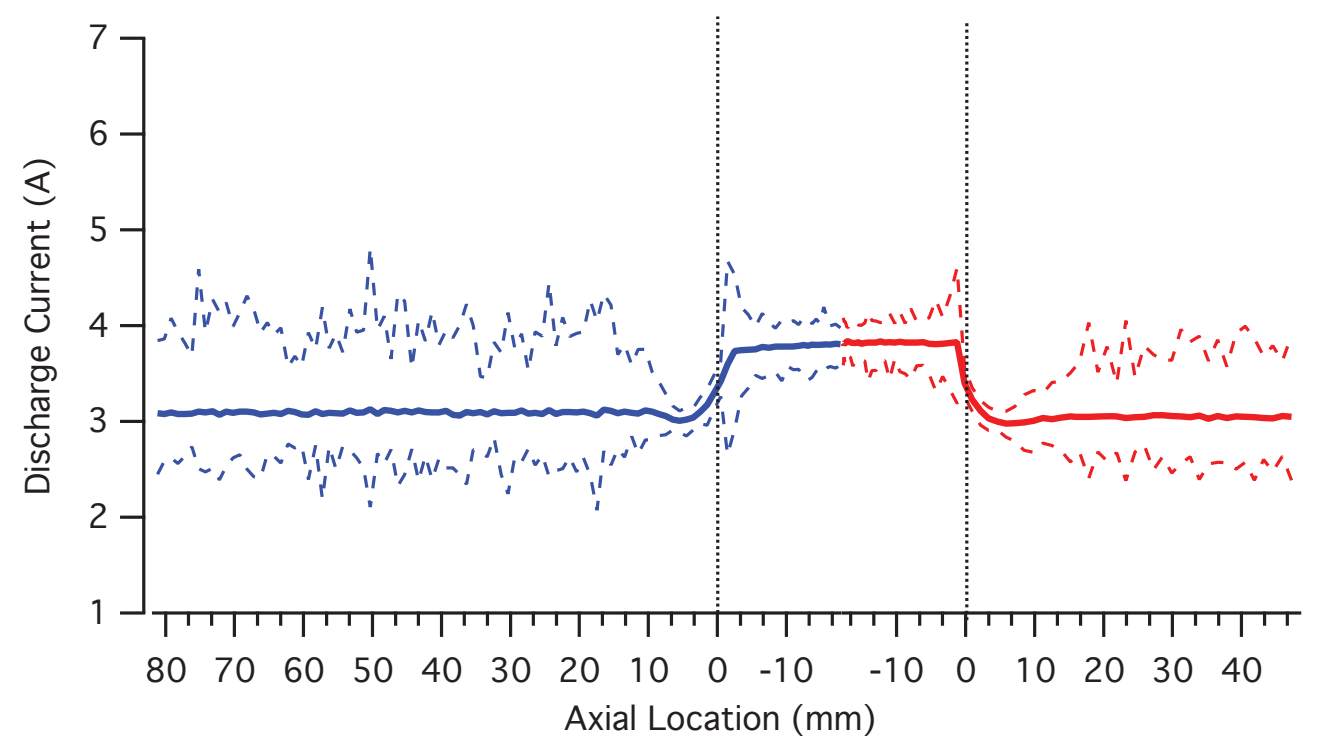

Figure 5.10: Discharge current measurements for xenon at $300 \mathrm{~V}$ as the Langmuir probe is inserted into the discharge chamber. The colored horizontal dashed lines represent the maximum and minimum current where the center bold line represents the average current. The vertical dotted lines represent the exit plane as its passed on the way into the discharge chamber and on the way back out. 
just $2 \mathrm{~mm}$ in front of the anode face.

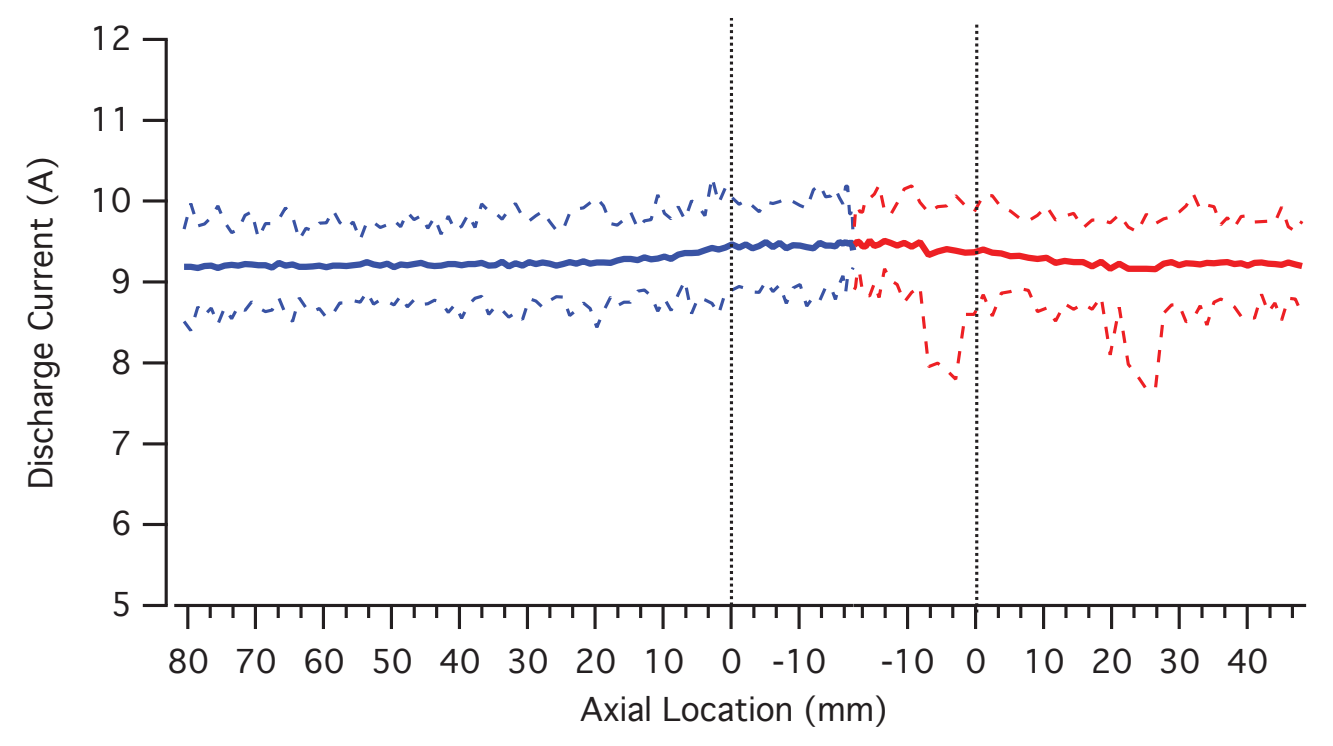

Figure 5.11: Discharge current measurements for krypton at $125 \mathrm{~V}$ as the Langmuir probe is inserted into the discharge chamber. The colored horizontal dashed lines represent the maximum and minimum current where the center bold line represents the average current. The vertical dotted lines represent the exit plane as its passed on the way into the discharge chamber and on the way back out.

It can be immediately seen that the probe's presence inside the discharge chamber is felt much more strongly in the high voltage cases than in the low voltage cases. This is most pronounced for xenon where the the measured increase in mean discharge current at $300 \mathrm{~V}$ for xenon was $22 \%$ while the probe was inside the discharge chamber. Oddly, this the exact number Linnel found as the largest perturbation in his high-speed probe work [5]. At low voltages, the increase was only $7 \%$. This measurement was taken by averaging the discharge current during a characteristic sweep and comparing the value against the average discharge current during normal operation without the probe.

Although the data shown in figures 5.10 and 5.12 doesn't appear to show a massive disturbance created by the probe, in watching the Hall thruster, a visual flash appeared to occur as the probe was leaving the discharge chamber for the higher discharge voltage cases. The data was recorded much faster than the eye can see and suggests the perturbations occur equally on the inward and outward stroke, contrary to what was visually observed. No flash was seen for the low 


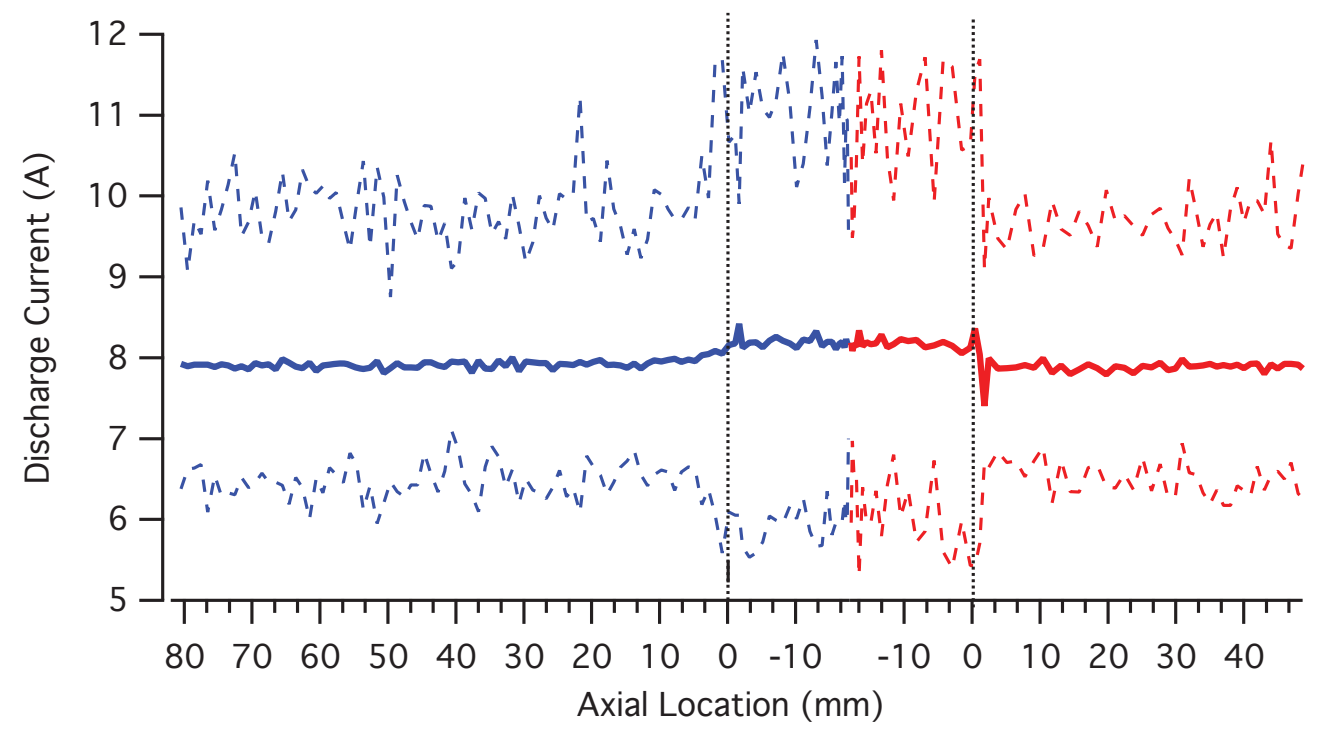

Figure 5.12: Discharge current measurements for krypton at $200 \mathrm{~V}$ as the Langmuir probe is inserted into the discharge chamber. The colored horizontal dashed lines represent the maximum and minimum current where the center bold line represents the average current. The vertical dotted lines represent the exit plane as its passed on the way into the discharge chamber and on the way back out. 
voltage cases, which is supported by the minimal disturbance as seen in figures 5.8 and 5.11 .

Linnel's high-speed probe work showed that the majority of his recorded discharge current perturbations were in the Hall current region after the probe had already passed through. He concluded that the perturbations may be less than feared, assuming that only the insertion portion of the probe data was analyzed. Nevertheless, his perturbations were also as high as $22 \%$ inside the discharge chamber.

Typical uncertainty values for analyzed internal probe data $\left(V_{p}, N_{e}\right.$, etc..) are on the order of $50 \%$ of the reported values, which is dominant over the error due to current perturbations [6]. Although the analysis of discharge current would suggest a higher degree of reliability in the analyzed data, it is believed that an error estimate of $50 \%$ is a safer and more reasonable assumption. 


\subsection{Summary}

The HPS was developed over a period of two years to meet the acceleration requirements necessary to limit the residence time of double Langmuir probe in the discharge chamber of the Hall thruster. A goal of $100 \mathrm{~ms}$ residence time was proposed and surpassed with the final product capable of a residence time $<60$ ms. Perturbation testing determined a $22 \%$ maximum change in discharge current during probe insertion into the discharge chamber which is lower than other published high-speed probe work. In addition to the motion table, a fully automated data acquisition system and data reduction software were engineered to acquire, store, and reduce the dataset. 
CHAPTER 5 


\section{Bibliography}

[1] Haas, J. M. and Gallimore, A. D., "Development of a High-Speed, Reciprocating Electrostatic Probe System for Hall Thruster Interrogation," Review of Scientific Instruments, Vol. 71, No. 11, November 2000.

[2] Haas, J. M., Low-Perturbation in Interrogation of the Internal and Near-Field Plasma Structure of a Hall Thruster Using a High-Speed Probe Positioning System, Ph.D. thesis, University of Michigan, 2001.

[3] Chen, F. F., "Lecture Notes on Langmuir Probe Diagnostics," IEEE-ICOPS, Jeju, Korea, June 52003.

[4] Chen, F. F., Introduction to Plasma Physics and Controlled Fusion, Plenum Press, 1984.

[5] Linnell, J. A. and Gallimore, A. D., "Internal Langmuir Probe Mapping of a Hall Thruster with Xenon and Krypton Prop," Vol. AIAA-2006-4470, Sacramento, California, 9-12 July 2006.

[6] Hutchinson, I. H., Principles of Plasma Diagnostics, Cambridge University Press, 1987. 
CHAPTER 5 


\section{Chapter 6}

\section{HPS Results}

This chapter details the analyzed results acquired by the high-speed probe system and a brief discussion. For details on the system itself or the data analyses methods see chapter 5.

\subsection{Test Matrix}

The original test plan included a $100 \mathrm{~V}$ discharge voltage trial for both propellants. In testing, the thruster struggled to remain on below $125 \mathrm{~V}$. Since the successful test set of data for the $100 \mathrm{~V}$ case was largely incomplete, it has been omitted. See table 6.1 for a complete list of the test operating conditions.

\subsection{Raw Results}

Three raw quantities are recorded during testing: floating potential of the sweep supply, sweep voltage of the supply, and current sourced/sinked by the supply. The data is recorded as a function of encoder clicks from which position can be derived.

Post processing was conducted by automated matlab scripts. The autonomous scripts first identify end of travel location within the dataset: $2 \mathrm{~mm}$ from the anode and $18 \mathrm{~mm}$ inside the exit plane. A total of $100 \mathrm{~mm}$ in axial space of data is then extracted from the recorded quantities for analyzing: $82 \mathrm{~mm}$ outside the discharge chamber and 18 inside. The rest of the recorded data is discarded. A sample of the sweep data and the section used for analysis is seen in figure 6.1. The matlab script then divides the data selection into individual double probe traces corresponding to one voltage sweep from $-40 \mathrm{~V}$ to $40 \mathrm{~V}$ as shown in figure 6.2. The individual traces are then evaluated as described in the previous chapter. 
Table 6.1: Test parameters for xenon and krypton

(a) Xenon test matrix

\begin{tabular}{|c|c|c|}
\hline$V_{d}(\mathrm{~V})$ & $I_{m}(\mathrm{~A})$ & $\dot{m}(\mathrm{mg} / \mathrm{s})$ \\
\hline 300 & 3.00 & 5 \\
\hline 300 & 2.50 & 5 \\
\hline 300 & 2.00 & 5 \\
\hline 300 & 1.50 & 5 \\
\hline 300 & 1.00 & 5 \\
\hline 200 & 3.00 & 5 \\
\hline 200 & 2.50 & 5 \\
\hline 200 & 2.00 & 5 \\
\hline 200 & 1.50 & 5 \\
\hline 200 & 1.00 & 5 \\
\hline 150 & 3.00 & 5 \\
\hline 150 & 2.50 & 5 \\
\hline 150 & 2.00 & 5 \\
\hline 150 & 1.50 & 5 \\
\hline 150 & 1.25 & 5 \\
\hline 150 & 1.00 & 5 \\
\hline 150 & 0.75 & 5 \\
\hline 150 & 0.50 & 5 \\
\hline 125 & 2.00 & 5 \\
\hline 125 & 1.50 & 5 \\
\hline 125 & 1.25 & 5 \\
\hline 125 & 1.00 & 5 \\
\hline 125 & 0.75 & 5 \\
\hline 125 & 0.50 & 5 \\
\hline 125 & 0.25 & 5 \\
\hline
\end{tabular}

(b) Krypton test matrix

\begin{tabular}{|l|l|l|}
\hline$V_{d}(\mathrm{~V})$ & $I_{m}(\mathrm{~A})$ & $\dot{m}(\mathrm{mg} / \mathrm{s})$ \\
\hline 300 & 3.00 & 5 \\
300 & 2.50 & 5 \\
300 & 2.00 & 5 \\
300 & 1.50 & 5 \\
300 & 1.00 & 5 \\
\hline 200 & 3.00 & 5 \\
200 & 2.50 & 5 \\
200 & 2.00 & 5 \\
200 & 1.50 & 5 \\
200 & 1.25 & 5 \\
200 & 1.00 & 5 \\
200 & 0.75 & 5 \\
\hline 150 & 3.00 & 5 \\
150 & 2.50 & 5 \\
150 & 2.00 & 5 \\
150 & 1.50 & 5 \\
150 & 1.25 & 5 \\
150 & 1.00 & 5 \\
150 & 0.75 & 5 \\
150 & 0.50 & 5 \\
\hline 125 & 1.25 & 5 \\
125 & 1.00 & 5 \\
125 & 0.75 & 5 \\
125 & 0.50 & 5 \\
125 & 0.25 & 5 \\
\hline
\end{tabular}


It was believed that the disturbance created in the plasma, by the presence of the probe, is less severe on the inward stroke than the outward stroke by simple virtue that the inwards stroke occurs first. Aside from asymmetries in the perturbations the dataset for the inward and outwards stroke should be identical. Thus, it was chosen analyze the inward bound portion of the sweep.

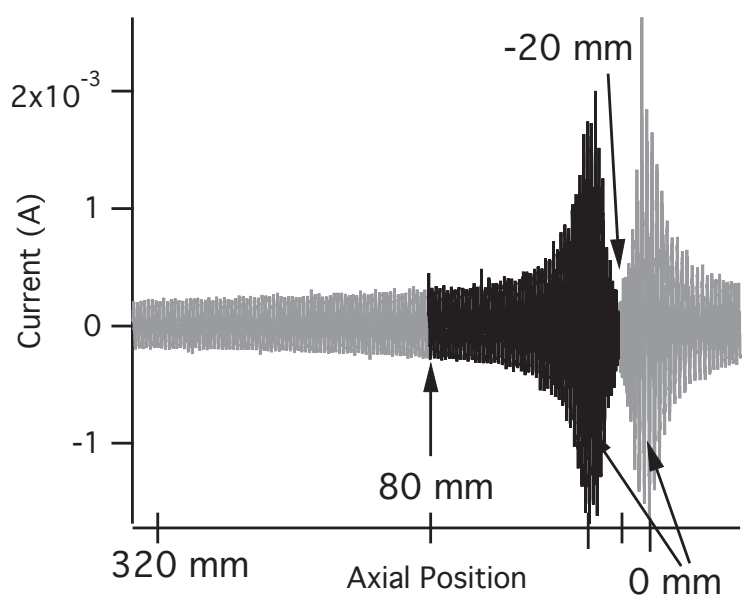

Figure 6.1: Raw current data as a function of axial position as recorded by the HPS. This is the entire recorded data set, however, only the portions in blue are used for data analysis. The blue data, and all red data preceding it represents the inward bound travel of the probe. $-20 \mathrm{~mm}$ (relative to the exit plane) is the end of travel where the probe stops just in front of the anode and reverses motion. All data to the right of the blue section represents the data during the return trip of the probe.

\subsection{Xenon Results}

Figures $6.3-6.10$ represent a selection of the fully analyzed data to illustrate certain trends. The complete results for the entire test matrix are presented in appendix A. A discussion of error analysis can be found in section 7.3. To avoid obscuring the data error bars have been omitted from the graphs. Plasma potential, floating potential, electron energy and ion density are represented in red, green, black and blue, respectively. The sample set in figures $6.3-6.10$ 


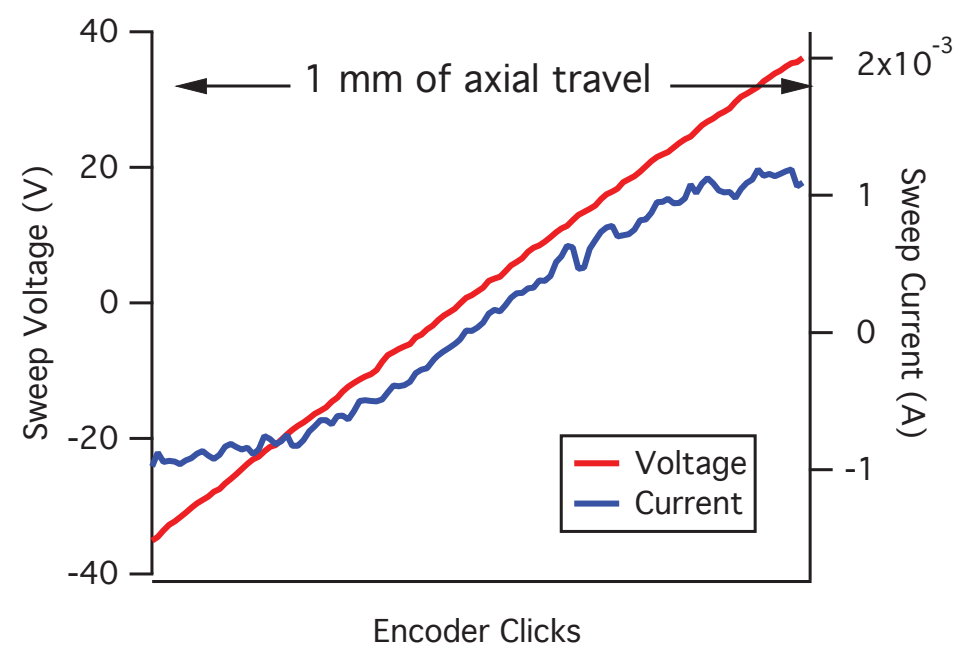

Figure 6.2: Typical example of raw current and voltage sweep data for a single extracted double probe trace. The motion table moves $1 \mathrm{~mm}$ during the signal acquisition. Data is shown before any manipulation or removal of the influence of line capacitance.

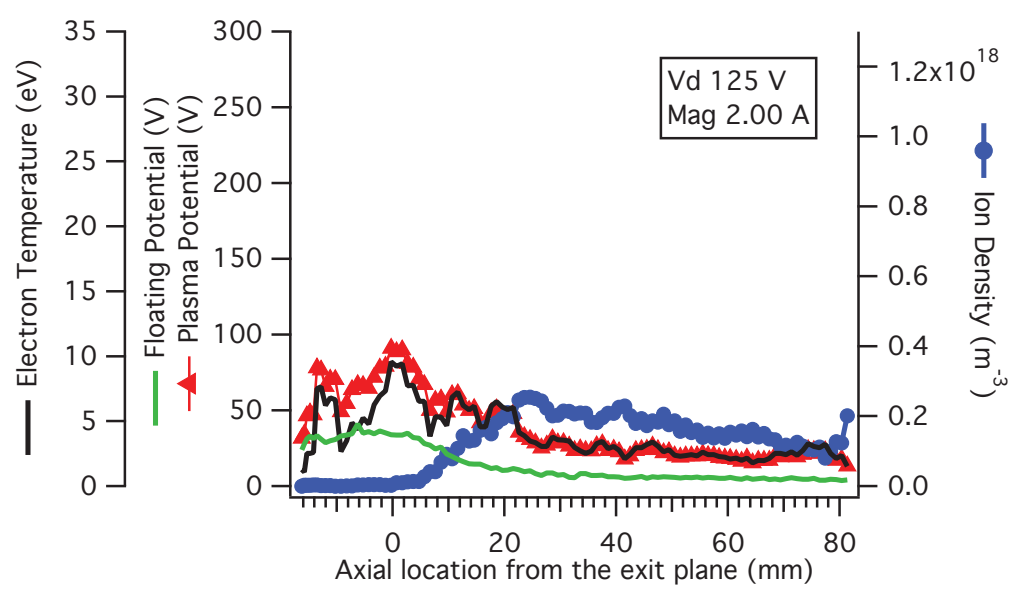

Figure 6.3: High-speed probe data for xenon 
was chosen here to exhibit the eight permutation samples of high/low discharge voltage, high/low magnet current, and propellant type.

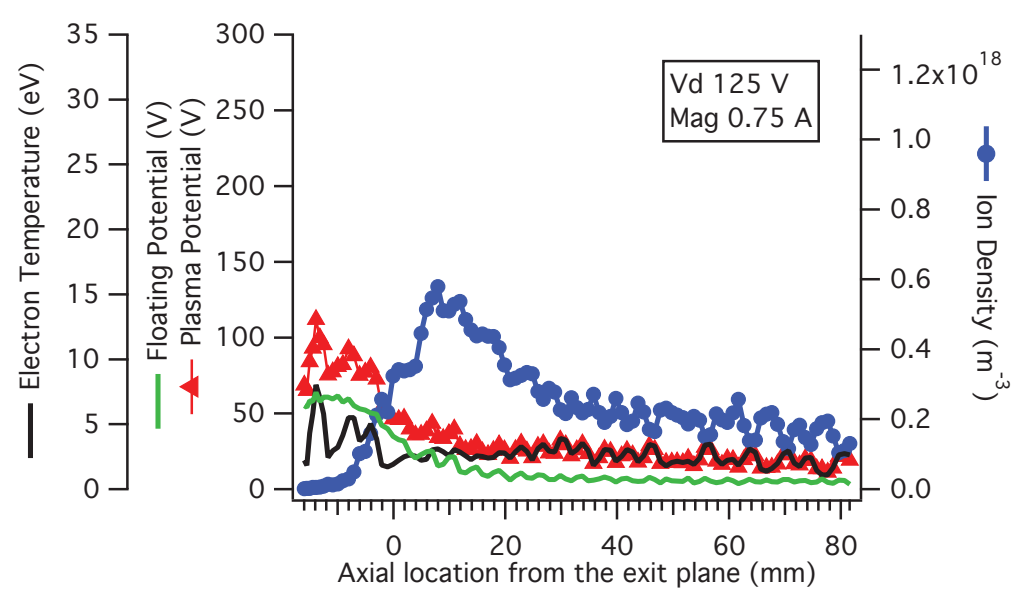

Figure 6.4: High-speed probe data for xenon

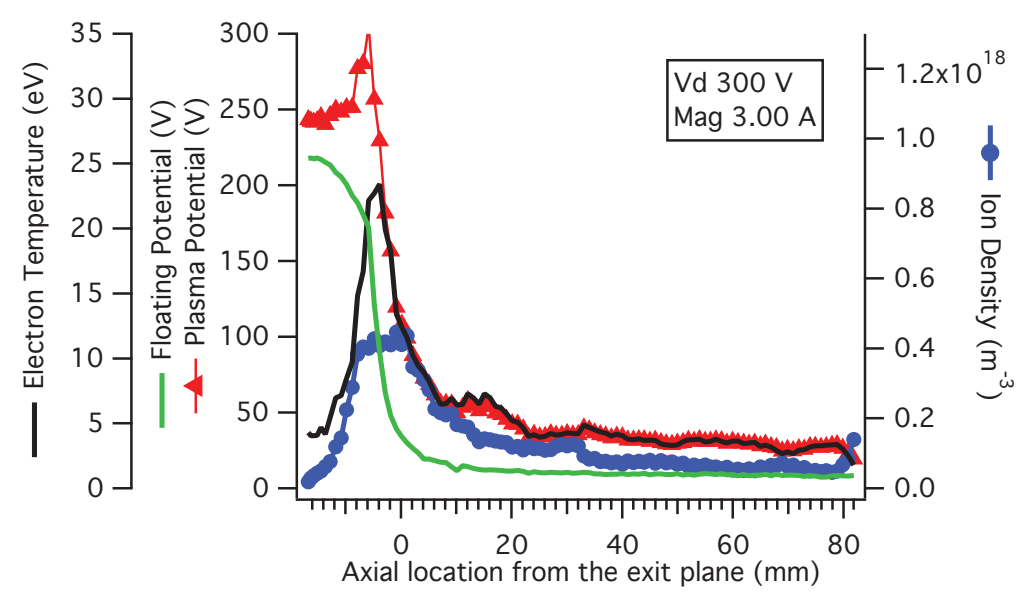

Figure 6.5: High-speed probe data for xenon 


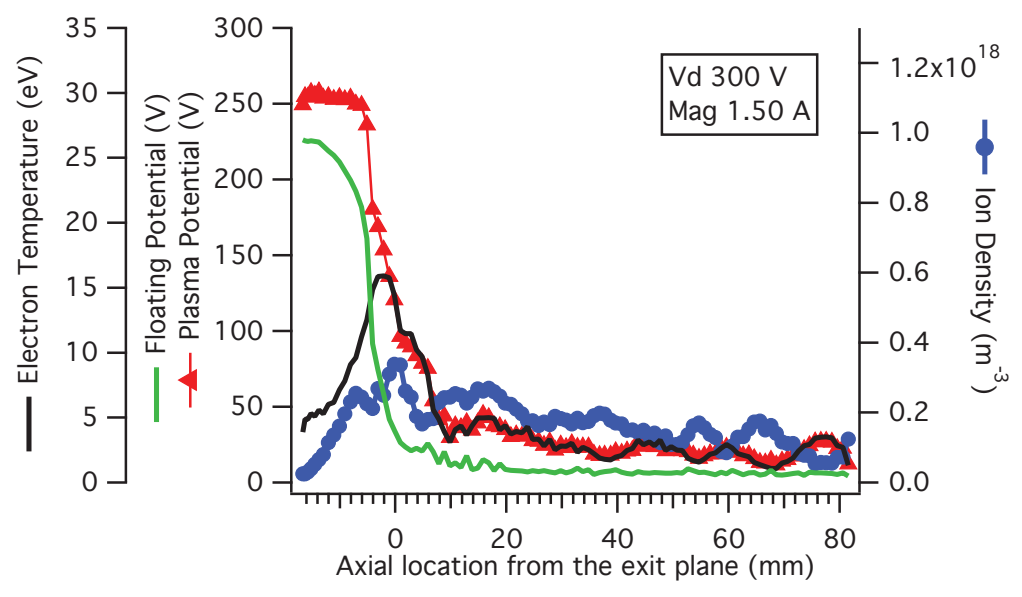

Figure 6.6: High-speed probe data for xenon 


\subsection{Krypton Results}

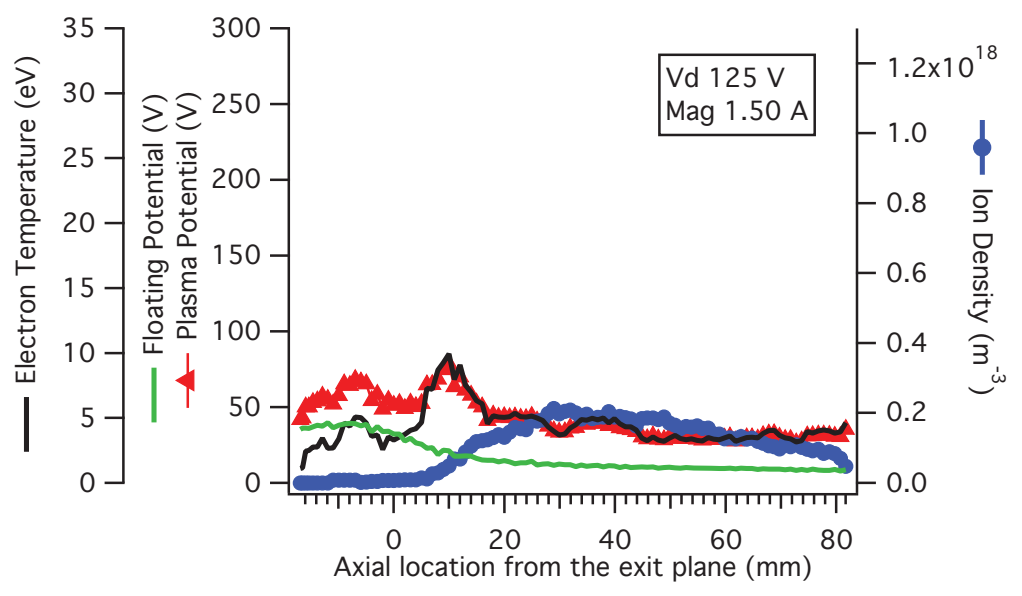

Figure 6.7: High-speed probe data for krypton

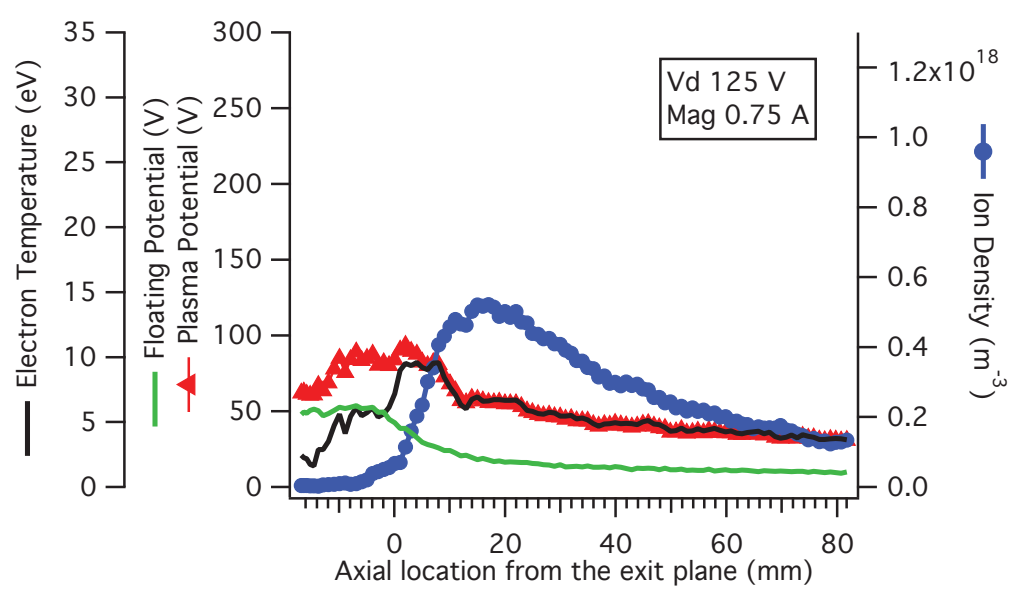

Figure 6.8: High-speed probe data for krypton 


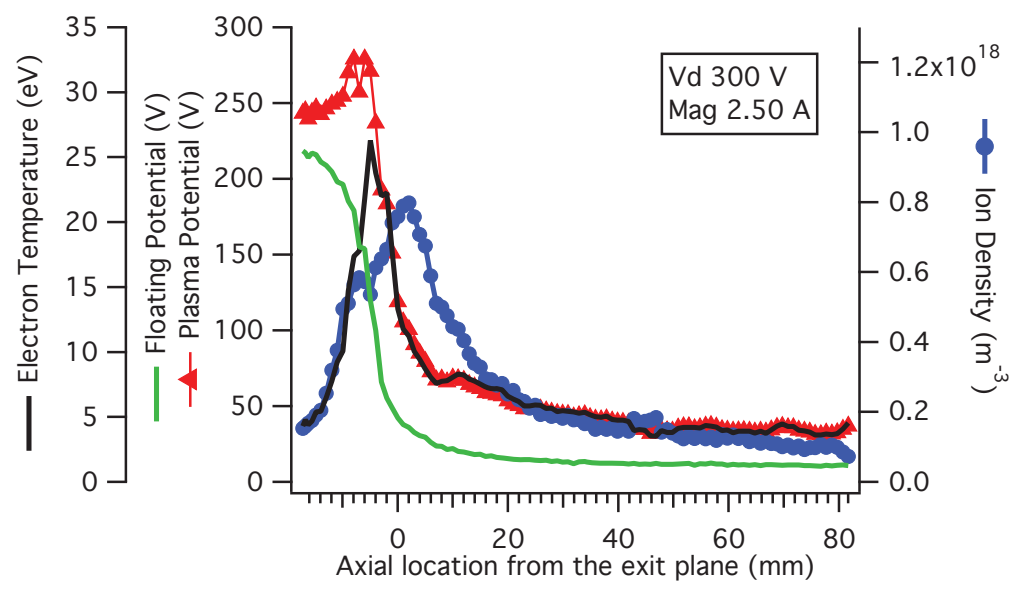

Figure 6.9: High-speed probe data for krypton

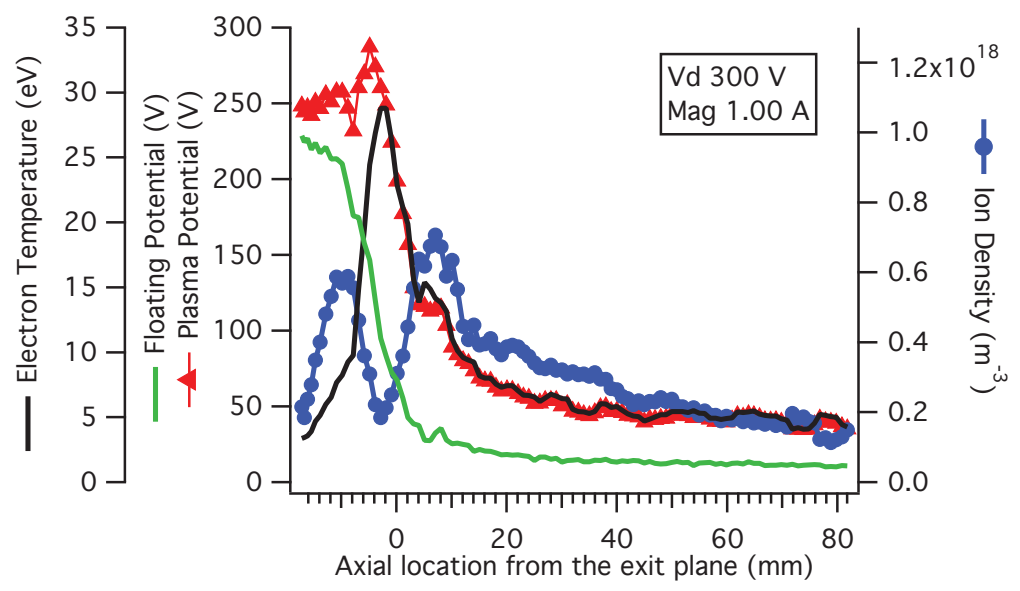

Figure 6.10: High-speed probe data for krypton 


\section{Overall trends and shape}

The change in magnet current seems to have a greater effect on the ion densities than it does on the plasma potentials. For both xenon and krypton at $125 \mathrm{~V}$, the higher magnet values push the maximum ion density further downstream such that the vast majority of the charged particles are outside of the discharge chamber. Very little ion density is recorded until about $5 \mathrm{~mm}$ past the exit plane for xenon and about $4 \mathrm{~mm}$ for krypton.

At $300 \mathrm{~V}$, neither propellant shows much movement in the acceleration zone location as a function of magnet current. The ion densities exhibit opposite movement, in comparison to the $125 \mathrm{~V}$ cases for both propellants, with increased magnet current causing a downstream shift in the ion density profile. Fo xenon, the peak ion density moves from upstream of the exit plane to downstream of it. The same is true for krypton, but can be seen to be even more pronounced (figures and 6.10).

\subsection{Ionization and Acceleration Zone locations}

In order to speak quantitatively on the effects of the magnet current on the plasma potential and ion density profiles, it is useful to mathematically define and identify what is known as the ionization zone and acceleration zone. The following text includes the definitions and some discussion of the limitations of this convention, the balance of which appears in section 7.3.

\section{Ionization Zone}

Theoretically, the ionization zone is the axial location of peak ion production for the thruster. Experimentally, the ionization zone is identified by the point of maximum current density $[1,2,3]$. Although current density and ion production, as a function of axial distance, are closely related, the point of peak ion production can occur upstream of peak ion density. Ion density is a function of charged particle flux. Therefore, the ion density differs from ion production location by the additional current recorded due to downward streaming ions created upstream of the probe location. To record ion production location is to record only newly born ions or those of zero velocity (aside from random thermal motion), which is something these probe techniques are incapable of. This uncertainty between the peak ion production zone and the peak ion density is discussed further in section 7.3. The ionization zone locations, as determined by the peak ion density, can be found in tables 6.2 and 6.3 . 
Table 6.2: Krypton Ionization Zone. Values are given in $\mathrm{mm}$ from the exit plane. Negative values indicate locations internal to the discharge chamber.

\begin{tabular}{|c|c|c|c|c|}
\hline Magnet Current (A) & $125 \mathrm{~V}$ & $150 \mathrm{~V}$ & $200 \mathrm{~V}$ & $300 \mathrm{~V}$ \\
\hline 0.50 & 16.2 & 9.2 & & \\
0.75 & 17.2 & 9.2 & -1.8 & \\
1.00 & 22.2 & -6.7 & -5.7 & 7.2 \\
1.25 & 30.1 & 1.2 & -0.8 & \\
1.50 & 29.1 & 0.2 & -1.8 & -7.7 \\
2.00 & & -4.8 & -2.7 & 2.2 \\
2.50 & & 29.1 & -3.8 & 2.2 \\
3.00 & & 30.1 & -1.8 & 2.2 \\
\hline
\end{tabular}

Table 6.3: Xenon Ionization Zone. Values are given in $\mathrm{mm}$ from the exit plane. Negative values indicate locations internal to the discharge chamber.

\begin{tabular}{|c|c|c|c|c|}
\hline Magnet Current (A) & $125 \mathrm{~V}$ & $150 \mathrm{~V}$ & $200 \mathrm{~V}$ & $300 \mathrm{~V}$ \\
\hline 0.50 & 15.1 & 4.1 & & \\
0.75 & 8.1 & 10.1 & & \\
1.00 & 8.1 & 9.1 & 6.1 & 9.1 \\
1.25 & 14.1 & 6.1 & & \\
1.50 & 19.1 & 2.1 & 6.1 & 0.2 \\
2.00 & 25.1 & 11.1 & 2.1 & 1.1 \\
2.50 & & 18.1 & 3.1 & 1.1 \\
3.00 & & 18.1 & 2.1 & -0.9 \\
\hline
\end{tabular}




\section{Acceleration Zone}

The location of the acceleration zone can be identified by the point of steepest slope in the plasma potential profile. Mathematically, this can be located by taking the peak of the derivative of the plasma potential profile. Due to the uncertainty in the electron temperature measurements from which the plasma potential is derived (see equation 5.7), the results of this approach were highly erratic. Therefore, the acceleration zone location was determined using the same mathematical approach described above using the floating potential data. Numerous other works have taken this same approach due to the reliability of the floating potential data and its likeness to the plasma potential $[4,5,6,7,8]$. The results from the plasma potential data were then compared to the floating potential data to establish a level of measurement uncertainty. Removing extreme outliers, the acceleration zone as determined by floating potential was on average $\pm 2 \mathrm{~mm}$ from the same calculation with the plasma potential data. The acceleration zone location results are in the tables 6.4 and 6.5.

Table 6.4: Krypton Acceleration region. Values are given in $\mathrm{mm}$ from the exit plane. Negative values indicate locations internal to the discharge chamber.

\begin{tabular}{|c|c|c|c|c|}
\hline Magnet Current (A) & $125 \mathrm{~V}$ & $150 \mathrm{~V}$ & $200 \mathrm{~V}$ & $300 \mathrm{~V}$ \\
\hline 0.50 & -0.1 & -1.5 & & \\
0.75 & 2.0 & 1.6 & -3.9 & \\
1.00 & 3.1 & 3.0 & -4.1 & -3.9 \\
1.25 & 3.0 & 1.8 & -2.6 & \\
1.50 & 4.0 & 4.3 & -3.1 & -4.7 \\
2.00 & & 5.3 & -3.3 & -4.9 \\
2.50 & & 5.3 & -2.5 & -5.0 \\
3.00 & & 6.8 & 2.9 & -4.8 \\
\hline
\end{tabular}

\subsection{Potential Drop in the Acceleration Zone}

In addition to the location of the ionization and acceleration zones, of equal importance to voltage utilization efficiency is the magnitude of the potential drop in the acceleration zone. This holds true, assuming any positively disproportionate amount of ion production occurs in the acceleration zone. For a given/fixed acceleration and ionization zone, if the maximum plasma potential in the acceleration 
Table 6.5: Xenon Acceleration Region. Values are given in $\mathrm{mm}$ from the exit plane. Negative values indicate locations internal to the discharge chamber.

\begin{tabular}{|c|c|c|c|c|}
\hline Magnet Current (A) & $125 \mathrm{~V}$ & $150 \mathrm{~V}$ & $200 \mathrm{~V}$ & $300 \mathrm{~V}$ \\
\hline 0.50 & -3.2 & -4.9 & & \\
0.75 & -0.1 & -3.8 & & \\
1.00 & 0.8 & 1.2 & -2.2 & -2.6 \\
1.25 & 4.6 & 3.8 & & \\
1.50 & 5.7 & 4.1 & -2.4 & -4.1 \\
2.00 & 9.6 & 5.7 & -3.2 & -4.0 \\
2.50 & & 8.6 & -3.2 & -4.8 \\
3.00 & & 7.7 & -3.1 & -3.9 \\
\hline
\end{tabular}

zone is decreased, the voltage utilization efficiency will decrease. The potential drop can be calculated by subtracting the minimum plasma potential as found $20 \mathrm{~mm}$ downstream ${ }^{1}$ from the exit plane from the maximum plasma potential inside the discharge chamber (or shortly outside of). The results are in figures 6.11 and 6.12 and it can be seen that the potential drop across the acceleration zone is minimally affected by the changing magnet current within the limits of the uncertainty.

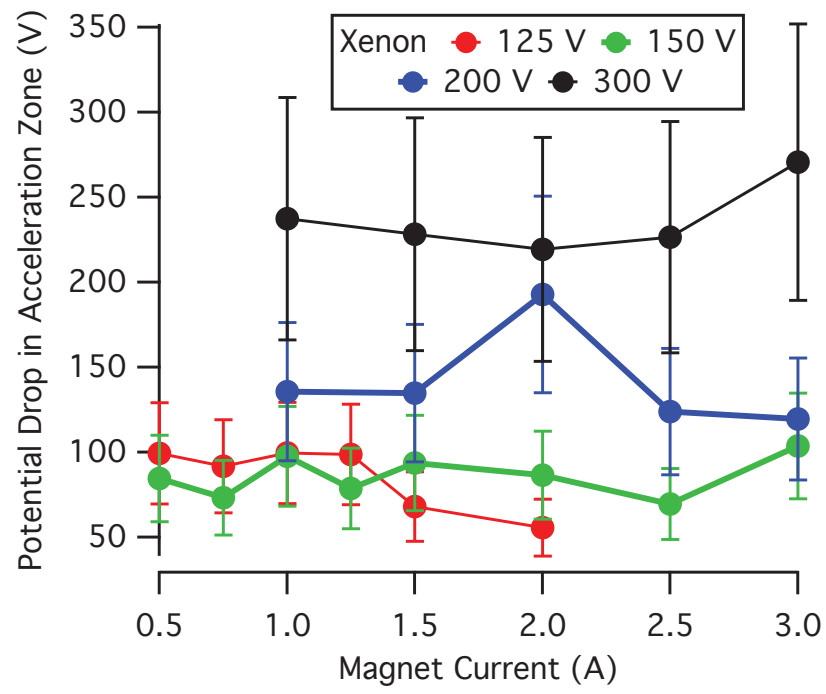

Figure 6.11: Potential drop across the acceleration zone for xenon

\footnotetext{
${ }^{1}$ The automated script actually searches for the minimum plasma potential value within \pm 3 $\mathrm{mm}$ from the $20 \mathrm{~mm}$ location to avoid any anomalous peaks
} 


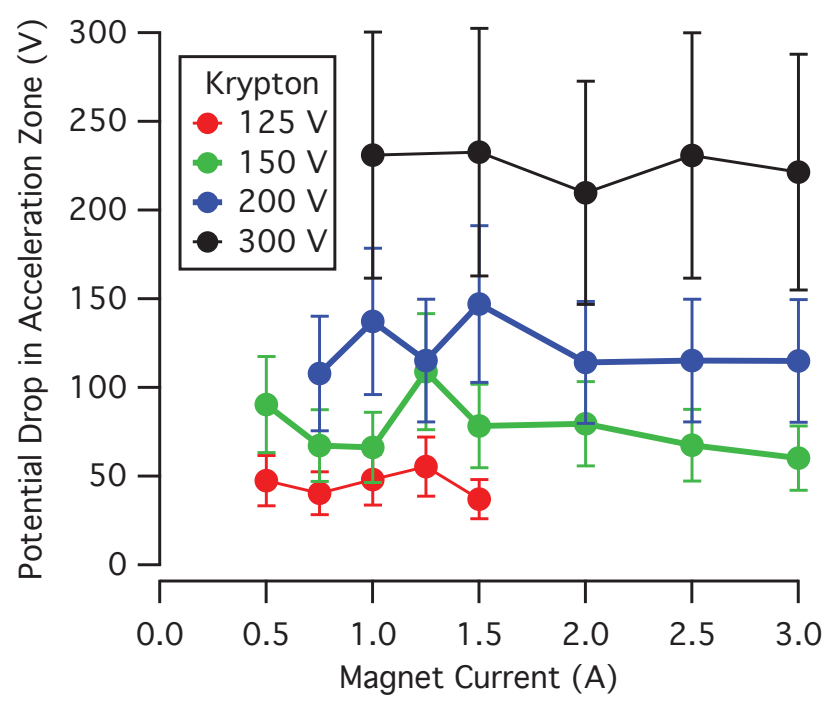

Figure 6.12: Potential drop across the acceleration zone for krypton

\section{Conclusions}

With the exception of a possible near-anode potential rise, it is not possible for the plasma potential to be increasing as a function of axial distance from the anode in a Hall thruster. In many of our experimental cases, however, the results show the plasma potential increasing downstream of the anode all the way out to exit plane (see figure 6.4). This is believed to be an artifact of the data, and is likely a byproduct of the uncertainty in the electron temperatures due to perturbation in the plasma caused by the insertion of the probe. As was shown in chapter 6 , the plasma potential is calculated from the electron temperature and floating potential as

$$
V_{p}=V_{f}+\frac{T_{e V}}{2} \ln \left(\frac{2 m_{i}}{\pi m_{e}}\right) .
$$

The electron energy measurements have at $30 \%$ uncertainty which propagates to an uncertainty of anywhere between $\pm 10 \mathrm{~V}$ to $\pm 80 \mathrm{~V}$ in the calculation of the plasma potential. The anomalous downstream rise in the plasma potential is therefore assumed to be the propagation of the electron temperature uncertainty. Figures 6.13 and 6.14 are example cases that display the plasma potential and its uncertainty plotted along side the floating potential and the electron temperature.

The ionization zone location is seen to be immobile for high voltages and magnet currents greater than $1.50 \mathrm{~A}$ for both propellants. The same can be said of 


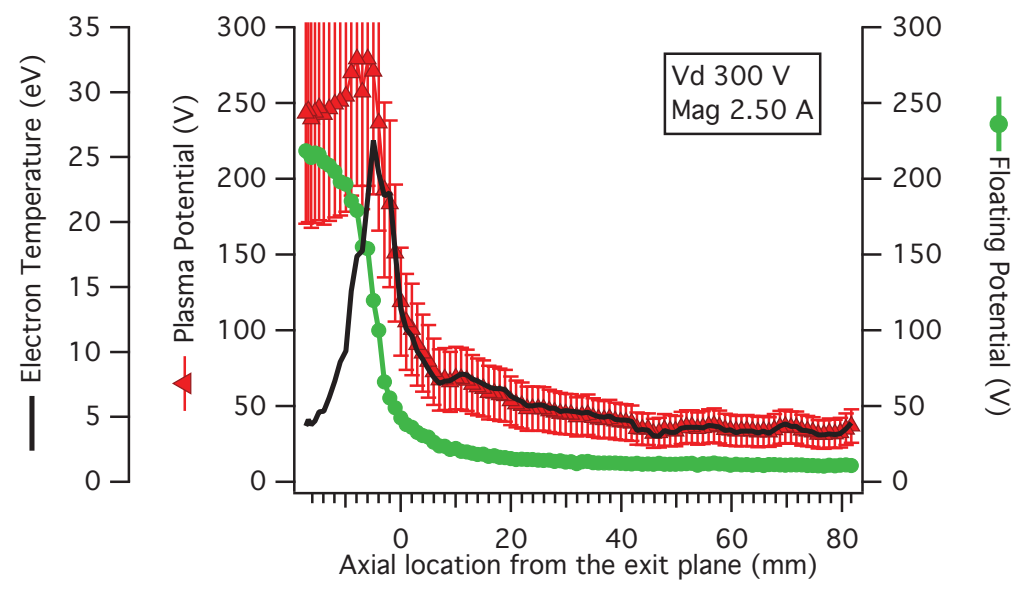

Figure 6.13: An example of floating potential, plasma potential and electron energy for krypton at $300 \mathrm{~V}$

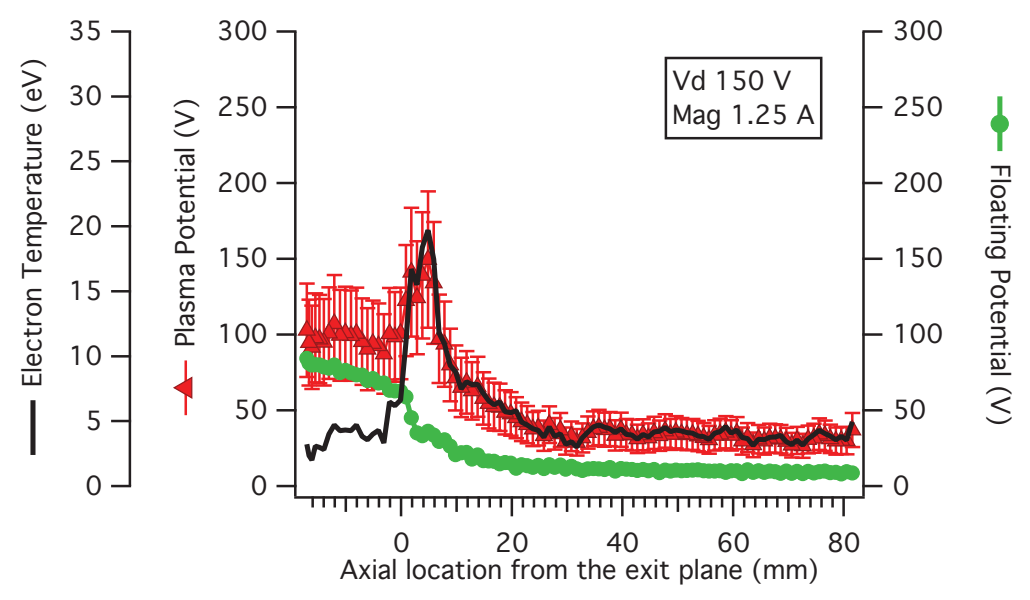

Figure 6.14: An example of floating potential, plasma potential and electron energy for krypton at $150 \mathrm{~V}$ 
the acceleration location. This indicates a lowered sensitivity in ion creation/acceleration dynamics on the magnetic field for nominal discharge voltages. In other words, axial position of the ionization processes are much more susceptible to changes in the magnetic field for low voltages than than they are for nominal/higher discharge voltages. This is in direct support of the external probe data found in the previous chapters. 


\section{Bibliography}

[1] Linnell, J. A. and Gallimore, A. D., "Internal Langmuir Probe Mapping of a Hall Thruster with Xenon and Krypton Prop," Vol. AIAA-2006-4470, Sacramento, California, 9-12 July 2006.

[2] Reid, B. M. and Gallimore, A. D., "Langmuir Probe Measurements in the Discharge Channel of a 6-kW Hall Thruster," 44th AIAA/ASME/SAE/ASEE Joint Propulsion Conference and Exhibit, Vol. AIAA-2008-4920, Hartford, CT, July 20-23 2008.

[3] Haas, J. M., Low-Perturbation in Interrogation of the Internal and Near-Field Plasma Structure of a Hall Thruster Using a High-Speed Probe Positioning System, Ph.D. thesis, University of Michigan, 2001.

[4] Kim, V., Grdlichko, D., Kozlov, V., Lazourenko, A., Popov, G., and Skrylnikov, A., "Local Plasma Parameter Measurements by Nearwall Probes Inside the SPT Accelerating Channel Under Thruster Operation with Kr," 38th AIAA/ASME/SAE/ASEE Joint Propulsion Conference 85 Exhibit, Indianapolis, Indiana, July 7-10 2002.

[5] Bishaev, A. M. and Kim, V., "Local Plasma Properties in a Hall-Current Accelerator with an Extended Acceleration Zone," Soviet Physics Technical Physics, Vol. 23, No. 9, 1978.

[6] Esipchuk, Y. B., Morozov, A. I., Tilinin, G. N., and Trofimov, A. V., "Plasma oscillations in closed-drift accelerators with an extended acceleration zone," Soviet Physics - Technical Physics, Vol. 18, No. 7, 1974.

[7] Raitses, Y., Ashkenazy, J., and Appelbaum, G., "Probe measurements of plasma properties inside an experimental Hall thruster," 34th AIAA/AS$M E / S A E / A S E E$ Joint Propulsion Conference and Exhibit, Cleveland, Ohio, July 13-15 1998. 
[8] Hofer, R. R., Development and Characterization of High-Efficiency, HighSpecific Impulse Xenon Hall Thrusters, Ph.D. thesis, University of Michigan, Ann Arbor, 2004. 


\section{Chapter 7}

\section{Discussion}

\subsection{Efficiency vs Discharge Voltage}

In the parametric investigation of chapter 3 , it was found that the excess current to the anode was the primary cause of inefficiency at low voltages. This is in direct agreement with the recent work by Dan Brown[1]. Additionally, it was noted that the traditional method of tuning the magnetic coils to obtain maximum thrust efficiency did not work. The assumption that is most often made is that minimum discharge current and maximum thrust efficiency are necessarily synchronous.

'Laboratory measurements are made by setting constant $V_{d}$ and $\dot{m}$, and then measuring thrust and $I_{d}$ after tuning the magnetic field strength and topology to minimize $I_{d}$ (or maximize $\eta_{T}$ ).' $[2]$

Minimum discharge current and maximum thrust efficiency did not coincide for many of the tests herein, as the above statement universally supposes it should. For many of the low voltage trials, the minimum discharge current and maximum thrust efficiency were mutually exclusive, a finding that is in conflict with existing data at nominal and higher discharge voltages [3, 4]. For these experiments, tuning the magnets to allow for additional current to the anode increased total efficiency. In Brown's low voltage work, he found a similar result and attested that the additional current was necessary to support ionization processes.

A summary of the findings from chapter 3 can be found in figures 7.1 -7.3. The figures depict the various efficiency terms for each mass flow rate as measured using probe techniques. As previously noted, the current efficiency becomes the dominant loss mechanism for all operation below $200 \mathrm{~V}$. The maximum thrust to power ratio recorded was $65 \mathrm{mN} / \mathrm{kW}$ at $250 \mathrm{~V}$. 


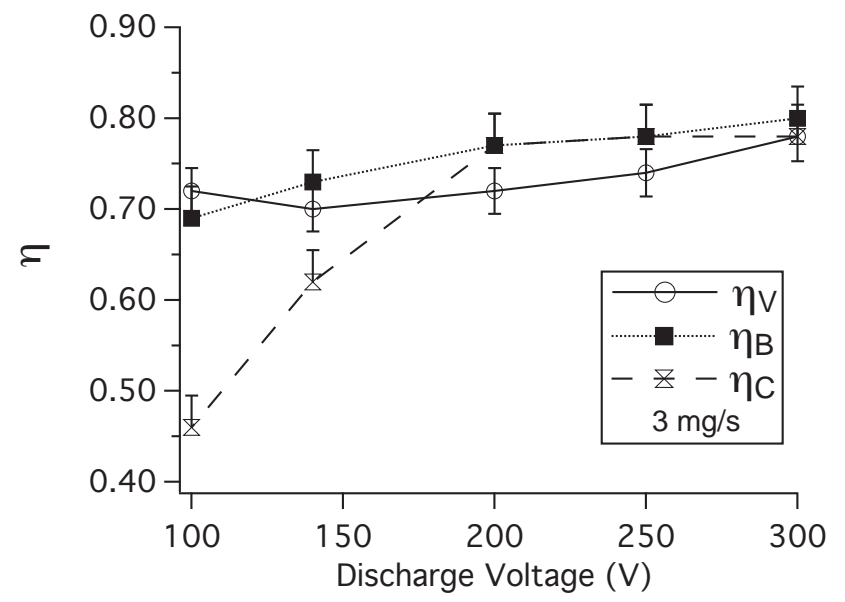

Figure 7.1: $\eta$ values for $3 \mathrm{mg} / \mathrm{s}$ of xenon at maximum thrust efficiency

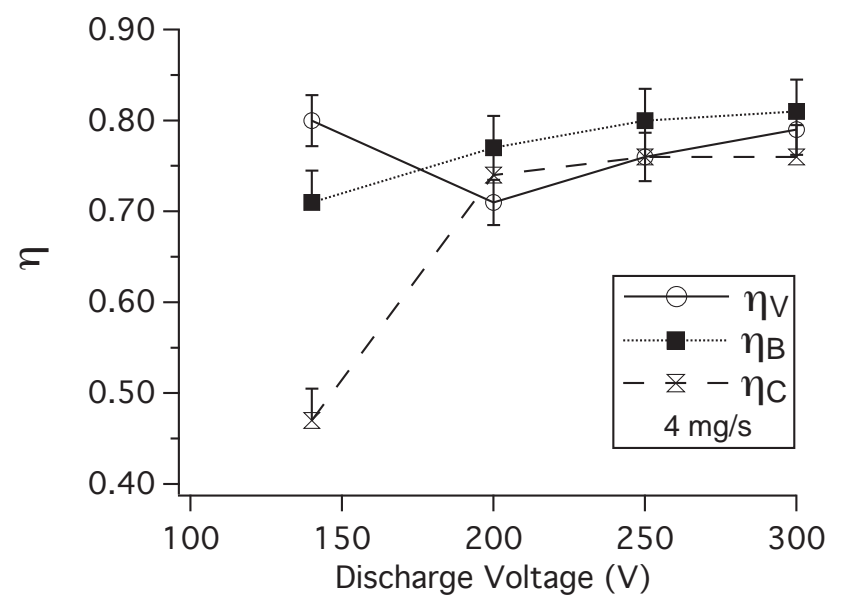

Figure 7.2: $\eta$ values for $4 \mathrm{mg} / \mathrm{s}$ of xenon at maximum thrust efficiency 


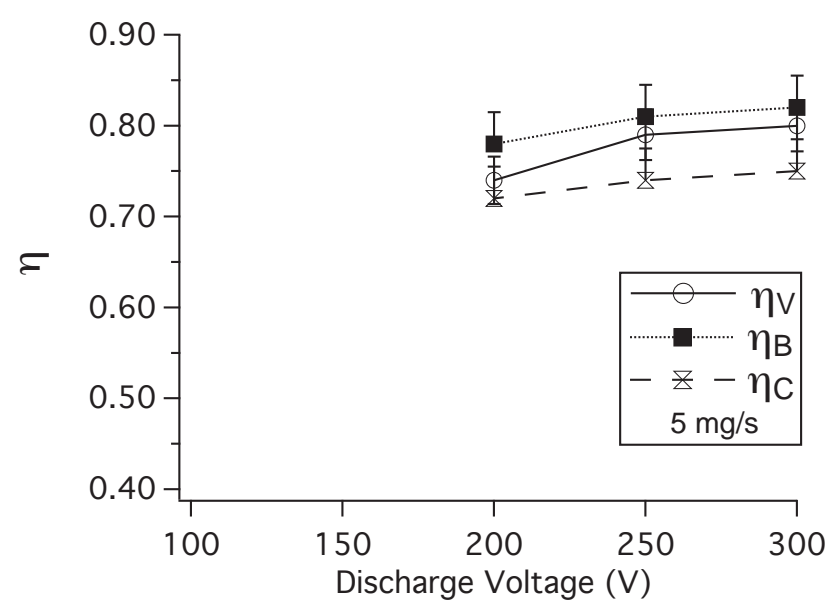

Figure 7.3: $\eta$ values for $5 \mathrm{mg} / \mathrm{s}$ of xenon at maximum thrust efficiency

In two data points (one of which fell inside the uncertainty) of these results showed voltage utilization efficiency exhibiting non-monotonic behavior as function of discharge voltage. The lowest discharge voltages, $100 \mathrm{~V}$ and $150 \mathrm{~V}$, showed an increase in $\eta_{v}$ at $3 \mathrm{mg} / \mathrm{s}$ and $4 \mathrm{mg} / \mathrm{s}$ flow rates respectively. Although far from conclusive, this was of interest because this has never been documented in other research and is in contrast to the behavior of the other loss mechanisms at low discharge voltage. The current efficiency and beam divergence efficiency monotonically decay with decreasing discharge voltage. Since it is well known that $\eta_{c}$ can be increased by adjusting the magnets to minimize the discharge current[2], this implies that one of the remaining loss mechanisms, beam divergence or current efficiency, is penalizing thrust efficiency as a function of increasing magnet current.

\subsection{Efficiency vs Magnet Current}

Chapter 3 measured loss mechanisms as a function of magnet current, or, off-peak thrust efficiency. Traditionally in the field data is primarily taken as function of peak thrust efficiency. This limits the correlations that can be made between operating characteristics and the strength of the magnetic field. Due to preliminary results, part of the hypothesis for this work was that high and low voltage operation would show contrasting changes in the operating characteristics as function of magnet current.

The chief results in chapter 4 are from figures 4.10 and 4.11 which show the voltage utilization as a function of magnet current for various discharge voltages. Surprisingly, the VUE is shown to increase for all voltages and for both propellants 
as a function of decreasing magnet current. This gives credence in the couple data points from chapter 3 that suggested that voltage utilization efficiency may favor low magnet current. This anomalous behavior is the strongest motivating element that necessitated internal probe studies.

Of interest to note, theoretical data published in 2004 presented a model that predicted the maximum voltage utilization would occur at $100 \mathrm{~V}$ discharge voltage. Discharge voltages above and below $100 \mathrm{~V}$ were shown to have lower $\eta_{v}$ values. No commentary was provided for this in their paper [5]. The data of chapter 4 disagrees with this showing the $100 \mathrm{~V}$ case having the lowest voltage utilization efficiency. Although the model suggests even lower voltages to show a decrease in VUE the results of this study do not explore discharge voltages below $100 \mathrm{~V}$ (due to thruster limitations) and therefore no comparisons can be made.

The need to reduce the strength of the magnetic coil current with decreasing discharge voltage in order to maximize thrust efficiency has been documented experimentally[6]. In previous work, the magnetic field value that produces the maximum thrust efficiency has deduced the empirical relationship

$$
p=\ln \left(B_{\max }\right) / \ln \left(V_{d}\right)
$$

where $B_{\max }$ is the peak magnetic field strength along thruster centerline and $p$ is a proportionality constant for a given thruster [5]. Values for the scaling relation $p$ have ranged from 0.5 - 1.5 for different thrusters and have shown to be invariant over a a wide range of voltages $[6,7]$. The proportionality is broken, however, at low voltages, which suggests a fundamental change in the physics of low voltage operation. To account for this, Ahedo's model for a standard Hall thruster predicted a scaling relation of 0.5 for nominal to high discharge voltages and 1 for lower voltages $\left(V_{d}<200 \mathrm{~V}\right)$.

To compare the results of this dissertation with Ahedo's model, the magnetic field strength must be known. Although Hall probe measurements of $B_{\max }$ were not taken in this study, magnetic field measurements have been taken before for nominal magnet currents $(2.5-3.0 \mathrm{~A})$ on this exact thruster[8]. From the magnetic field data at nominal operation, we can predict the field at lower currents using the Biot-Savart's law

$$
B=\frac{\mu_{0} I}{4 \pi R^{2}} \oint d L
$$

which dictates that the magnetic field is linearly proportional to the current through the coils, barring saturation. In the aforementioned study, it was shown that the magnetic field was below the saturation point and thus we can model $p$ based on the current through the magnetic coils since our magnet current levels are less than or equal to the values used for the gauss measurements $[9,8]$. The results for the proportionality constant are plotted in figure 7.5. The magnetic 
field probe used to make the measurements is accurate to $0.1 \mathrm{mT}$ which gives a $<1 \%$ uncertainty on $p$.

Although neither propellant exhibits entirely monotonic behavior, the trend is that with decreasing discharge voltage comes a decrease in $p$. With only three data points for xenon, not much can be concluded. Krypton shows a bit more of a developed trend, but also lacks in a significant quantity of data. For both propellants, the $150 \mathrm{~V}$ case had an unusually high $p$ that disrupts the trend. If the downward trend in $p$ value with decreasing discharge current is to be believed, then this data disagrees with Ahedo's model. Part of the boundary conditions of the model constrains the possible ionization and acceleration zones to locations inside of the discharge chamber. As was seen in chapter 6, and discussed further in section 7.3, these zones were experimentally found to occur, at times, external to the discharge chamber. Consequently, the steadfast boundary conditions may be prohibiting the model from accurately capturing the physics of low voltage operation and therefore correctly predicting the $p$ value.

\subsection{High-speed probe test}

In chapter 5 equation 5.1 stated that thin sheath limit was an appropriate method for $\xi$ values $\gg 1$.

$$
\begin{array}{r}
\xi \gg 1 \text { thin sheath limited analysis } \\
\quad \xi \ll 1 \text { orbit motion limited }
\end{array}
$$

A refinement to the rule of $\xi \gg 1$, Chen states it is more ideal to use orbit motion limit theory (OML) when the $\xi<3$ and thin sheath limit when $\xi>10$ [12]. Figure 7.6 shows our calculated values for $\xi$. Although $\xi>1$ at all locations, it is rarely $>10$ and occasionally $<3$. As such, a decision was made to evaluate the data using both TSL and OML and to take a weighted average of the two values based on the calculated $\xi$. A weighted average, or weighted mean, is a mathematical operation that is used to create a hybrid result of two separate calculations based on a weighting criteria. In this case, the individual OML and TSL results are weighted by $\xi$ such that the greater $\xi$ is, the closer the final values for $n_{e}, V_{p}$, etc. are to the TSL results. Conversely, the lower $\xi$ is, the closer the final values are to the OML results. This approach has also been used by Reid from the Plasmdynamics \& Electric Propulsion Laboratory at the University of Michigan [10].

The magnitude error is estimated as $30 \%$ for electron energy values and $50 \%$ for the ion density values $[13,14]$. All conclusions, however, are drawn from the 


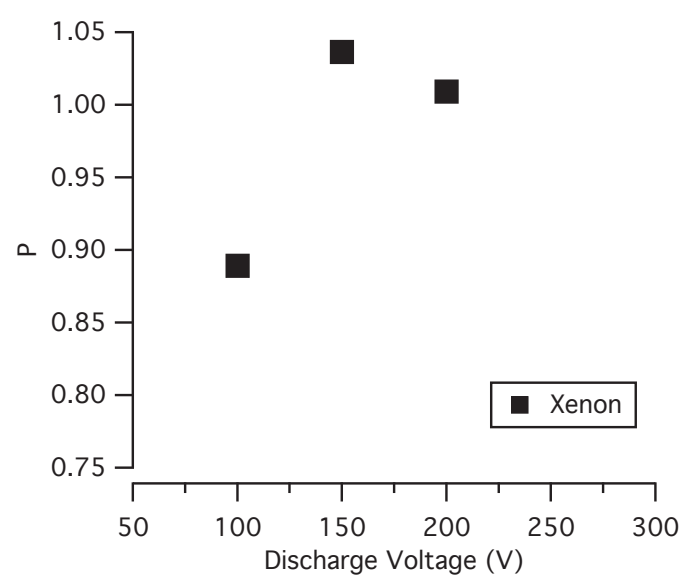

Figure 7.4: Magnetic $p$ value for xenon

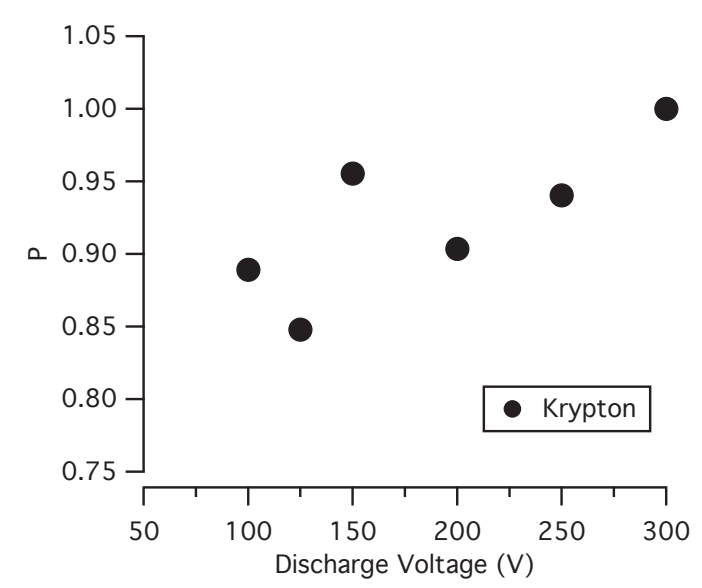

Figure 7.5: Magnetic $p$ value for krypton

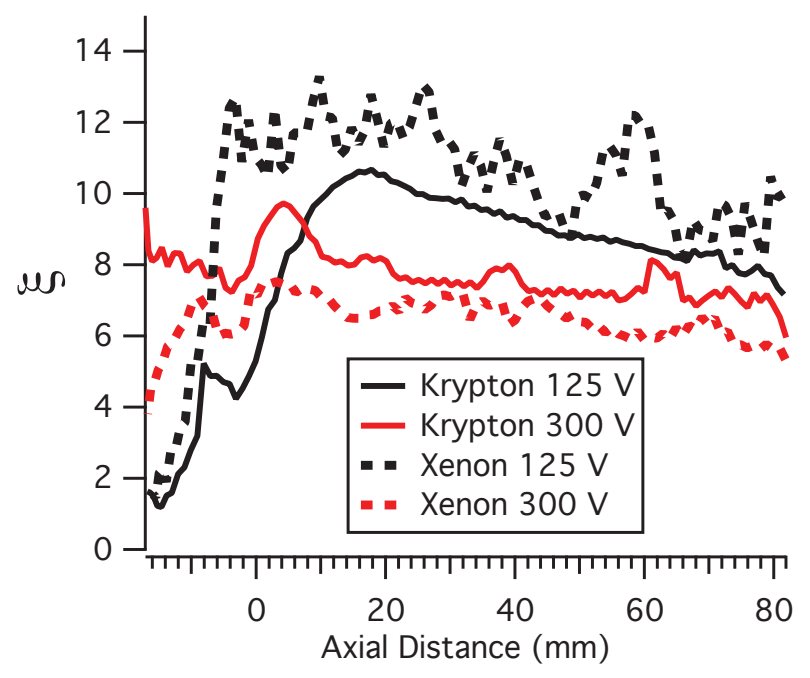

Figure 7.6: $\xi$ Values for high/low voltages and both propellants 
shape of the profile of the data and not the magnitudes. For both propellants, lowering the magnet current has the effect of increasing the plasma density and pushing it backward into the discharge chamber. Although the upstream shift is visually apparent in figures 7.7 and 7.8 , the calculation of the ionization zone, seen in figures 7.12 and 7.14 , does not show the same result. This is ramification of detecting the ionization zone by the peak in the ion density profile, as discussed in chapter 6. For many cases, this is a solid approximation, however, for cases like xenon at $125 \mathrm{~V}$ with $0.50 \mathrm{~A}$ magnet current (figure 7.9 ), the peak of the ion density profile concludes that the ionization region is $15.1 \mathrm{~mm}$ downstream of the exit plane. Upon visual inspection of figure 7.9, it is clear that much of the ionization region occurs near the exit plane.

In chapter 4 it was shown that voltage utilization efficiency increases with decreasing magnet current for both propellants (figures 4.10 and 4.11). The voltage utilization of an ion is dependent on where it is ionized along the potential structure created by the anode-cathode voltage. Collisions will also have a small energy-robbing effect on an ion, but the bulk of an ion's exit velocity is determined by the portion of the anode potential it sees. There are only a few possibilities that would lead to an increase in voltage utilization:

- The potential drop in the acceleration zone could be increasing (assuming any portion of the ion production occurs in, or upstream, of the acceleration zone).

- The ionization zone is moving upstream with respect to the acceleration zone.

The latter hypothesis could involve both regions moving in either direction, upstream or downstream, but the net effect must be that the ionization zone is further upstream with respect to the acceleration zone to see an increase in the voltage utilization. The results discount the first hypothesis and support the second, see figures $7.11-7.14$. Note that negative values indicate a distance in $\mathrm{mm}$ inside the discharge chamber from the exit plane and positive values are distances away from the exit plane.

The uncertainty in the acceleration location is taken to be $\pm 2 \mathrm{~mm}$ (see section 6.5). The unknown error in the ionization zone location measurement arises from the fact that the peak ion density is not necessarily the location of peak ion production. Although it is reasonable to assume that the peak ion density marks the end of the ion production region, the peak of ion production could occur further upstream than the peak ion density. Therefore, as a conservative error 


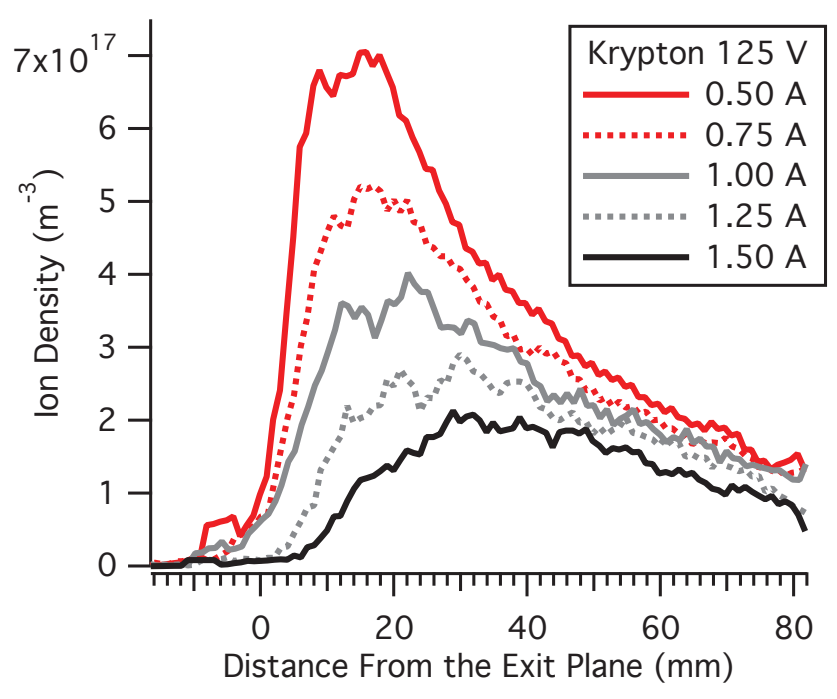

Figure 7.7: Krypton ion density

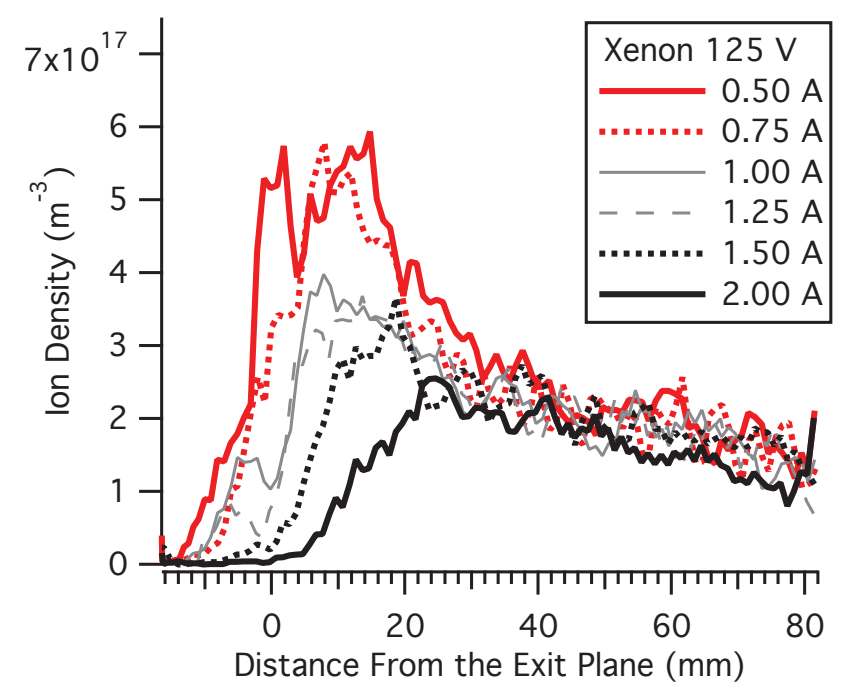

Figure 7.8: Xenon ion density 


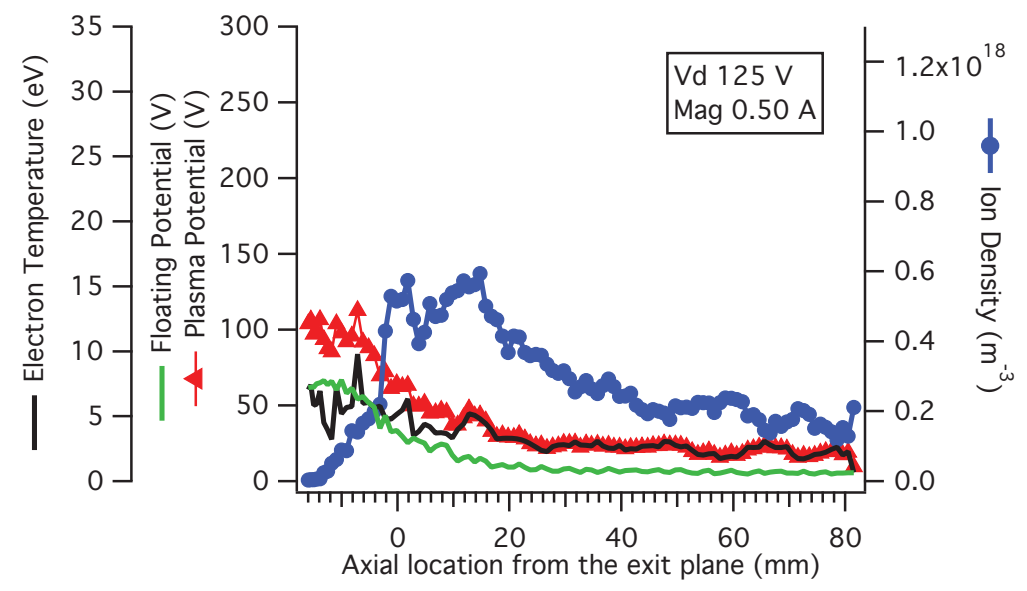

Figure 7.9: High-speed probe results for xenon at $125 \mathrm{~V} 0.50 \mathrm{~A}$

estimate, if the distance is measured over which the ion density is increasing (ion production region), it can be assumed that the peak in ion production occurs in the later half of this distance. In other words, it is assumed that the ion production region begins inside the discharge chamber where the current density is non-trivial $\left(>0.1 \times 10^{18} \mathrm{~m}^{-3}\right)$, and ends at the peak of the ion density profile. ${ }^{1}$ Half of this distance is taken as the uncertainty in the ionization zone location as a one-sided error bar in the upstream direction from the stated ionization zone location. Figure 7.10 is a graphical example of the uncertainty region as determined by the above criteria. Incidentally, even if the true ionization zone (peak ion production location) coexists with the peak of the ion density profile, there remains an additional uncertainty error due to the probe positioning system. This error, however, is less than $1 \mathrm{~mm}$ in either direction.

For most operating conditions, the beginning of the ion production region, as determined by the first downstream location from the anode of non-trivial current density $>0.1 \times 10^{18} \mathrm{~m}^{-3}$, begins at the closest location to the anode measured, $-18 \mathrm{~mm}$ from the exit plane. This is not universal, however, as several of the low voltage cases do not show signs of significant ion production until further downstream towards the exit plane.

There is no appreciable change in the fraction of the discharge voltage drop in the acceleration zone as a function of magnet current for any discharge voltage

\footnotetext{
${ }^{1}$ It should be noted that trace levels of ionization will occur on either side of the bounds as defined here, but will be small in comparison
} 
Table 7.1: The upstream uncertainty in the ionization zone location as measured from the peak ion density toward the anode

\begin{tabular}{|c|c|c|c|c|}
\hline Discharge Voltage & $125 \mathrm{~V}$ & $150 \mathrm{~V}$ & $200 \mathrm{~V}$ & $300 \mathrm{~V}$ \\
\hline \hline Xenon & $13 \mathrm{~mm}$ & $11 \mathrm{~mm}$ & $10 \mathrm{~mm}$ & $7 \mathrm{~mm}$ \\
\hline Krypton & $13 \mathrm{~mm}$ & $10 \mathrm{~mm}$ & $7 \mathrm{~mm}$ & $9 \mathrm{~mm}$ \\
\hline
\end{tabular}

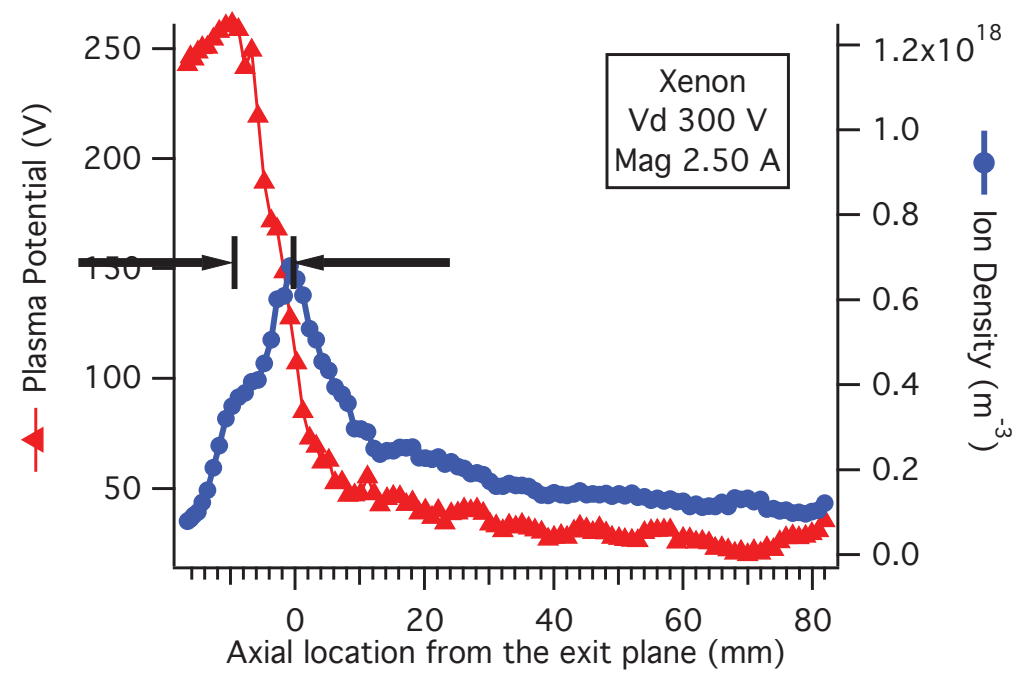

Figure 7.10: An example of the uncertainty in the ionization zone location as shown between the black arrows 


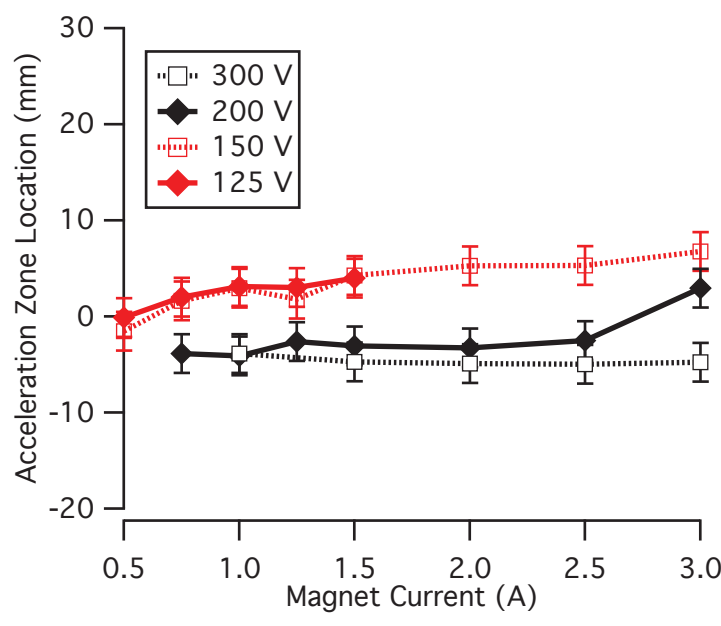

Figure 7.11: Acceleration zone location as a function of magnet current for krypton

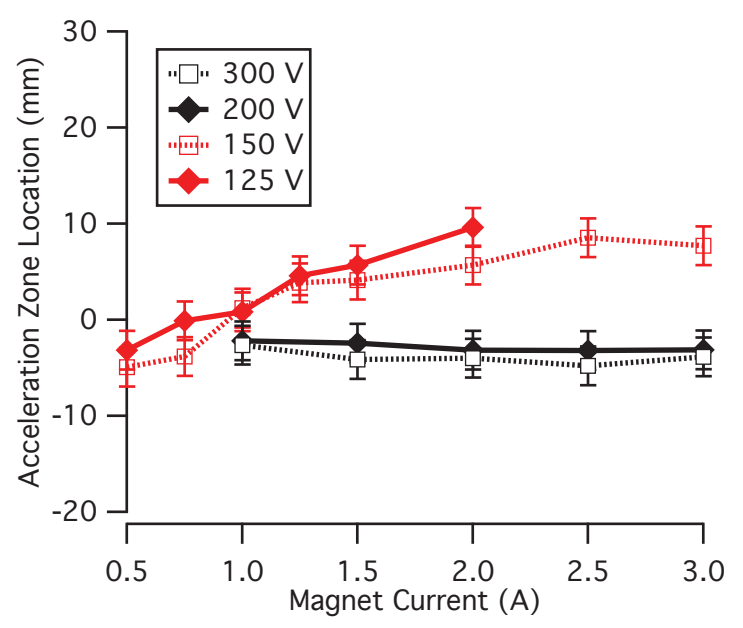

Figure 7.13: Acceleration zone location as a function of magnet current for xenon

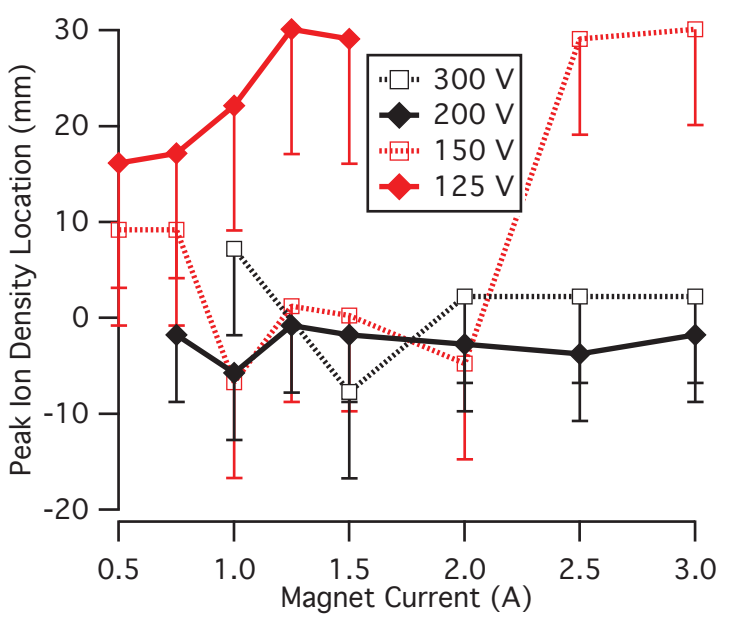

Figure 7.12: Ionization zone location as a function of magnet current for krypton

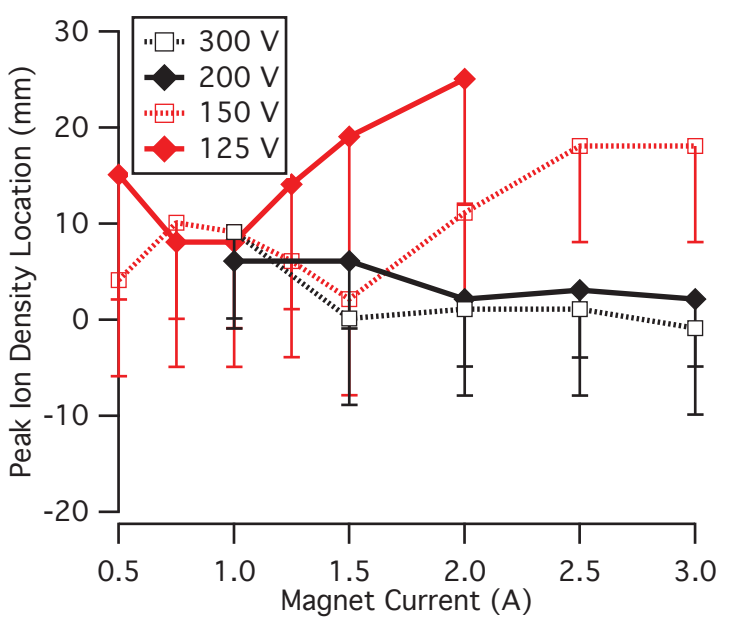

Figure 7.14: Ionization zone location as a function of magnet current for xenon 
as shown in chapter 6 . As a function of magnet current, however, there is an axial shift in both the acceleration and ionization zone locations in the upstream direction for low voltage trials. This shift is greater for the ion density profile, driving the ionization zone upstream towards the anode.

For xenon at higher voltages, the acceleration zone location is moved upstream with decreasing magnet current for the $200 \mathrm{~V}$ case and and ever so slightly downstream in the $300 \mathrm{~V}$ case. For krypton, neither the $200 \mathrm{~V}$ or $300 \mathrm{~V}$ cases show much sensitivity in acceleration zone location as a function of magnet current. Within the limits of the measurement uncertainty, no discernible trends are evident in the ionization zone location for the $200 \mathrm{~V}$ and $300 \mathrm{~V}$ trials for either propellant.

It is interesting to note that in the low voltage xenon trials, the acceleration zone location remains further downstream of the Hall thruster for all magnetic current values as compared to the higher voltage cases. The same is true for krypton with the exception of magnet current values $<0.5 \mathrm{~A}$ where the acceleration zone moves far enough upstream to match the location exhibited by the higher voltage trials. In general, the ionization zone location is also further downstream for the low voltage cases for both propellants. 


\subsection{Separation Distance}

Figures 7.15 and 7.16 below show the separation distance between ionization zone and acceleration zone locations. The distance is calculated as the acceleration zone location subtracted from the ionization zone location. The result is that positive values indicate an ionization zone location downstream of the acceleration zone location and the contrary for negative separation values. Unfortunately, due to the error bars, very little can be ascertained from the separation datasets. One dataset for krypton, however, remains statistically significant. The lowest discharge voltage case, $125 \mathrm{~V}$, does exhibit a decreasing separation distance as a function of decreasing magnet current. This 'closing of the gap' between the ionization zone and acceleration zone supports the increase in voltage utilization efficiency seen in the RPA probe results of chapter 4, figure 4.11. The numerical data for the separation distances are in tables 7.2 and 7.3

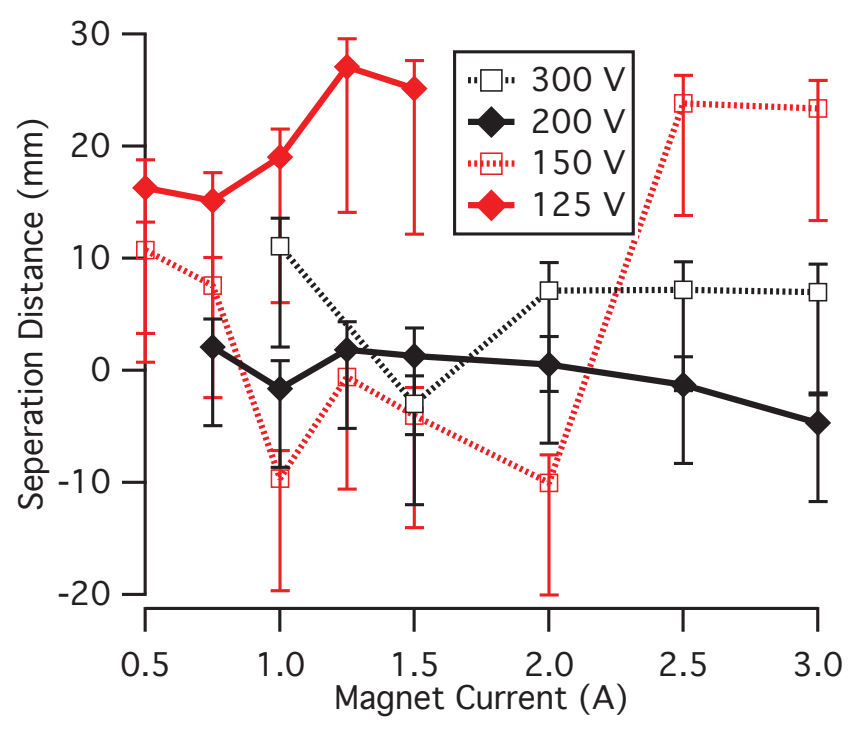

Figure 7.15: The separation distance between the acceleration zone and the ionization zone for krypton. Negative values indicate an ionization zone location upstream of the acceleration zone. 


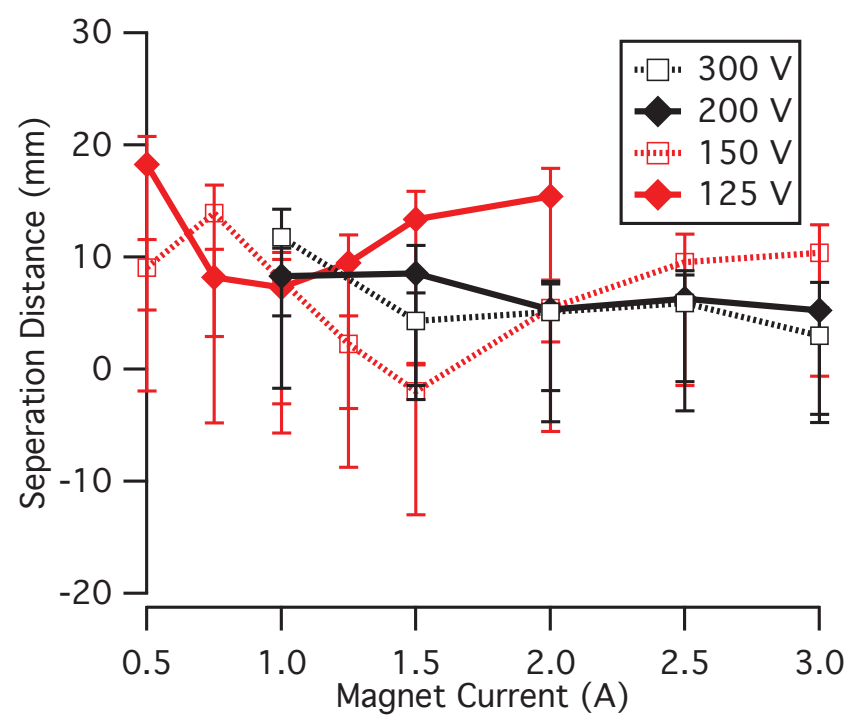

Figure 7.16: The separation distance between the acceleration zone and the ionization zone for xenon. Negative values indicate an ionization zone location upstream of the acceleration zone.

Table 7.2: The separation distance between the acceleration zone and the ionization zone for krypton. Negative values indicate an ionization zone location upstream of the acceleration zone.

\begin{tabular}{|c|c|c|c|c|}
\hline Magnet Current (A) & $125 \mathrm{~V}$ & $150 \mathrm{~V}$ & $200 \mathrm{~V}$ & $300 \mathrm{~V}$ \\
\hline 0.50 & 16.3 & 10.7 & & \\
0.75 & 15.3 & 7.6 & 2.1 & \\
1.00 & 19.0 & -9.7 & -1.6 & 11.1 \\
1.25 & 27.1 & -0.6 & 1.8 & \\
1.50 & 25.1 & -4.0 & 1.3 & -3.0 \\
2.00 & & -10.1 & 0.5 & 7.1 \\
2.50 & & 23.8 & -1.3 & 7.2 \\
3.00 & & 23.4 & -4.7 & 7.0 \\
\hline
\end{tabular}

\subsection{Magnetic Field}

Changing the current in the magnetic coils will linearly amplify or attenuate the magnet field profile in the Hall thruster. So far, the ionization and acceleration locations have been shown to move axially with changing magnet current. By comparing the magnetic field profile to the ionization and acceleration locations for various magnet current levels, it can be determined if the axial shift in these 
Table 7.3: The separation distance between the acceleration zone and the ionization zone for xenon. Negative values indicate an ionization zone location upstream of the acceleration zone.

\begin{tabular}{|c|c|c|c|c|}
\hline Magnet Current (A) & $125 \mathrm{~V}$ & $150 \mathrm{~V}$ & $200 \mathrm{~V}$ & $300 \mathrm{~V}$ \\
\hline 0.50 & 18.3 & 9.1 & & \\
0.75 & 8.2 & 13.9 & & \\
1.00 & 7.3 & 7.9 & 8.3 & 11.8 \\
1.25 & 9.5 & 2.3 & & \\
1.50 & 13.4 & -2.0 & 8.6 & 4.3 \\
2.00 & 15.4 & 5.5 & 5.3 & 5.1 \\
2.50 & & 9.6 & 6.3 & 5.9 \\
3.00 & & 10.4 & 5.3 & 3.0 \\
\hline
\end{tabular}

regions corresponds to a shift to a preferential magnetic field strength.

By a combination of Sommerville's measured and modeled data, a magnetic field centerline profile can be made for the BPT-2000 as seen in figure 7.17 [8]. With the magnetic field data as a function of coil current in hand, we can calculate

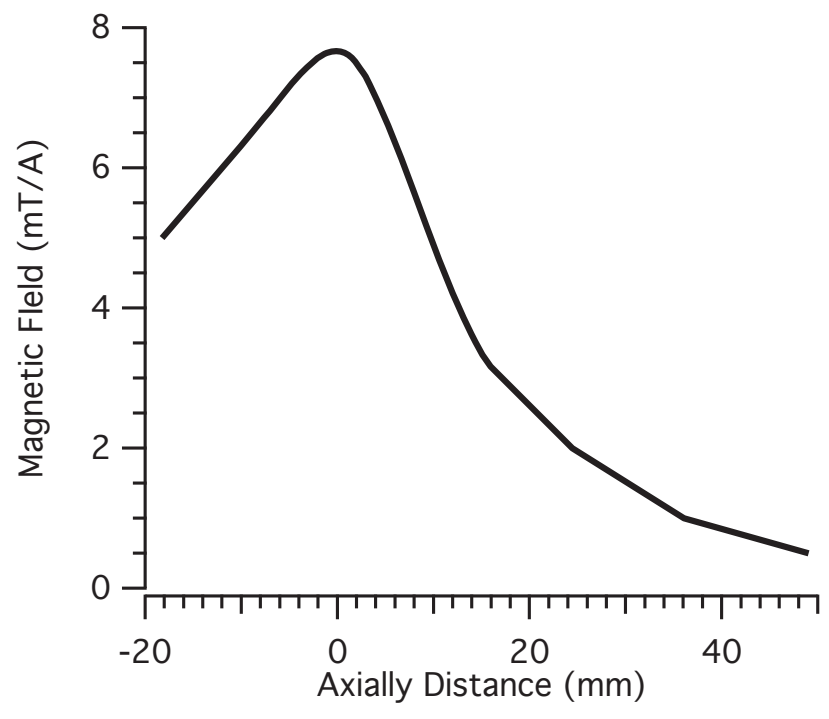

Figure 7.17: Axial magnetic field profile for the BPT-2000 along centerline

the magnetic field at the acceleration and ionization zone location as seen in figures 7.18 - 7.21. The uncertainty in the gauss probe measurements is $0.1 \mathrm{mT}$ and $1 \%$ on the coil current measurements. Therefore, the primary uncertainty in figures 7.18 
- 7.21 is in the location of the ionization and acceleration zones, which propagates to the uncertainty in the spatial location where the magnetic field value is assessed.

The values for both the ionization zone location and acceleration zone location are highly linear for both propellants at both 200 and $300 \mathrm{~V}$. In the lower voltage cases, the trends are linear at the lower magnet current values, but deviate as the magnet current is increased. For krypton's $125 \mathrm{~V}$ test case, the ionization zone location is nearly constant at 20 gauss for all magnet current values. This suggests that the zone location is occurring at a particular magnetic field value independent of where it may exist in axial space. The $150 \mathrm{~V}$ case for krypton follows the trends of the higher voltages until the magnet current is increased above 2.0 A where the ionization zone location moves to a region of much lower magnetic field strength more in line with the $125 \mathrm{~V}$ case.

The xenon data for the ion zone location seems to level out in field strength for both of the lower voltage cases as the magnet current increases. The field strength at the point of ionization zone location for the higher voltage cases continues to climb linearly over all of the magnet current values tested.

It is of little surprise, that the higher discharge voltage cases follow linear trend in figures $7.18-7.21$ if they are compared to figures 7.11-7.14. Neither the ionization zone location nor the acceleration zone location exhibited a large change in axial location as a function of magnet current. Since the magnetic field strength necessarily increases linearly with changing magnet current, the less movement in the locations of the ionization zone and acceleration zone, with respect to magnet current values, the more linear the trends of figures $7.18-7.21$ will be.

\subsection{Internal VS External}

To compare the external voltage utilization data of chapter 4 with the high-speed internal probe data of chapter 6 is not exactly straight forward. To replicate a voltage utilization value with internal data, one would need the exact birth location of each ion and the corresponding plasma potential at that location. The weighted average of the two variables would yield the voltage utilization efficiency (see equation 7.5). Plasma potential, as a function of linear space, is one of the recorded quantities of the internal probe study (albeit with a $\pm 30 \%$ uncertainty). Ion birth location, however, is not. Instead, Langmuir probes are limited to ion density, which although is closely related to the ionization birth location, is a measure of ion flux. Nevertheless, with the plasma potentials and ion densities,

a crude measurement of an internal voltage utilization efficiency, iVUE, can be 


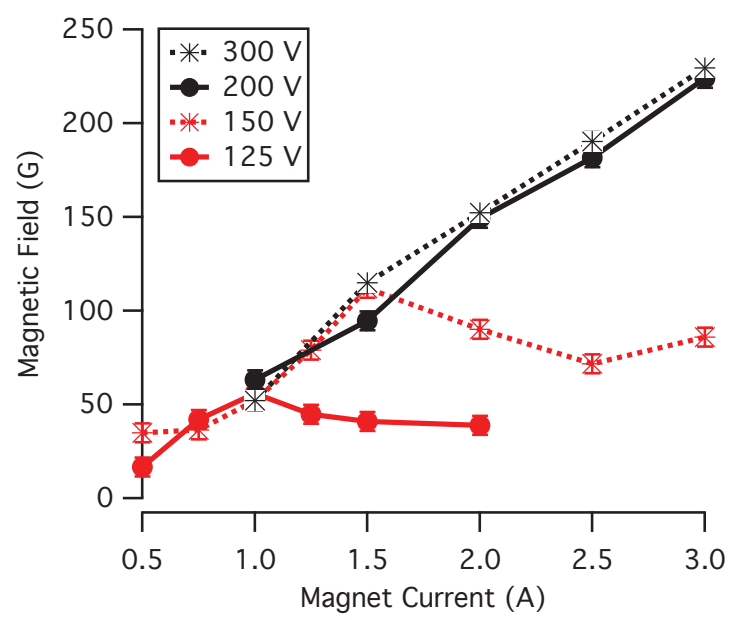

Figure 7.18: Magnetic field strength at the peak ion density location for xenon

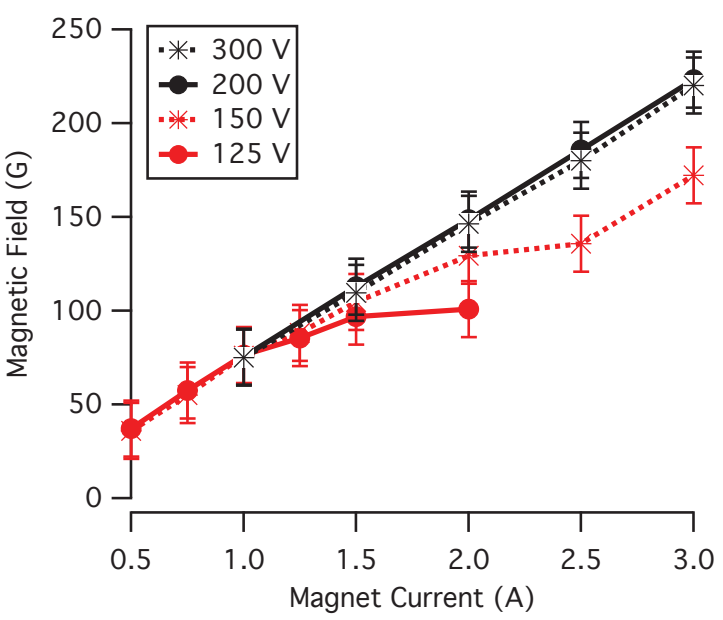

Figure 7.19: Magnetic field strength at the acceleration zone location for xenon

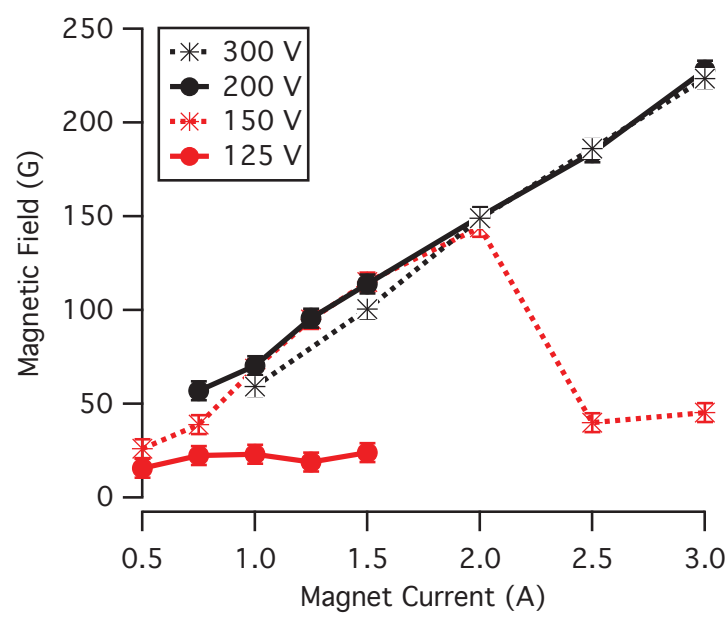

Figure 7.20: Magnetic field strength at the peak ion density location for krypton

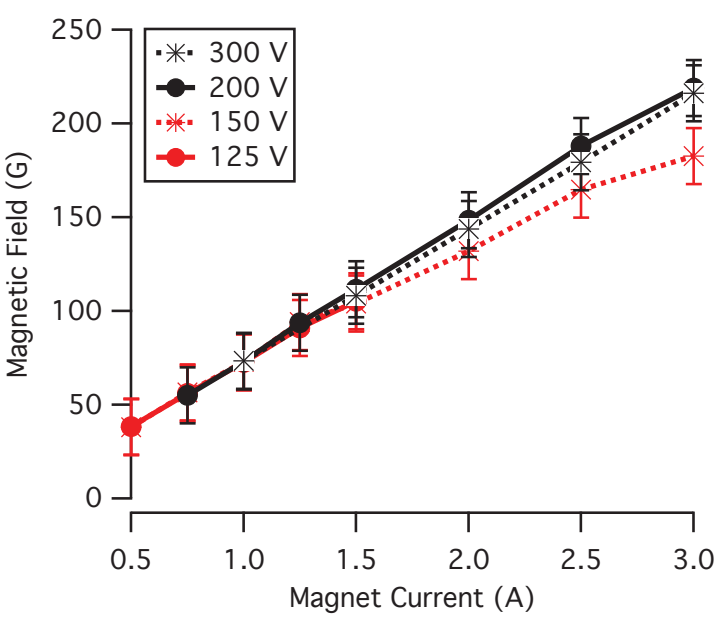

Figure 7.21: Magnetic field strength at the acceleration zone location for krypton 
created.

The internal voltage utilization efficiency was calculated assuming that the peak in the ion density curve represented the end of the ion production region. Because of the nature of the weighted average function, we can assume the ionization region begins at the deepest point recorded, $-18 \mathrm{~mm}$, without any mathematically penalty. Thus, for a given discharge voltage, the internal voltage utilization efficiency was calculated by taking a weighted average of all ion density measurements from the peak inward to the anode, as can be seen in the equation 7.5 .

$$
i V U E\left(V_{d}\right)=\frac{\int V_{p}(x) \cdot N_{e}(x) d x}{V_{d} \int N_{e}(x) d x}
$$

Figure 7.22 shows a green box indicating the region over which the automated scripts took the weighted average. Alternative to the weighted average approach, the reader can immediately see the pitfall in assuming that all of the ionization occurs only at the peak ion density. This would suggest that all ions are born at $100 \mathrm{eV}$ plasma potential and would result in an 30\% voltage utilization efficiency by negating the ion production upstream of the peak. Because of the flux of ions created upstream from the location the double probe collects them, the ion density profile will be biased downstream from that of a true ion production location profile. Consequently, we expect the internal voltage utilization efficiency to be a lower bound on the voltage utilization efficiency as calculated by the RPA probe.

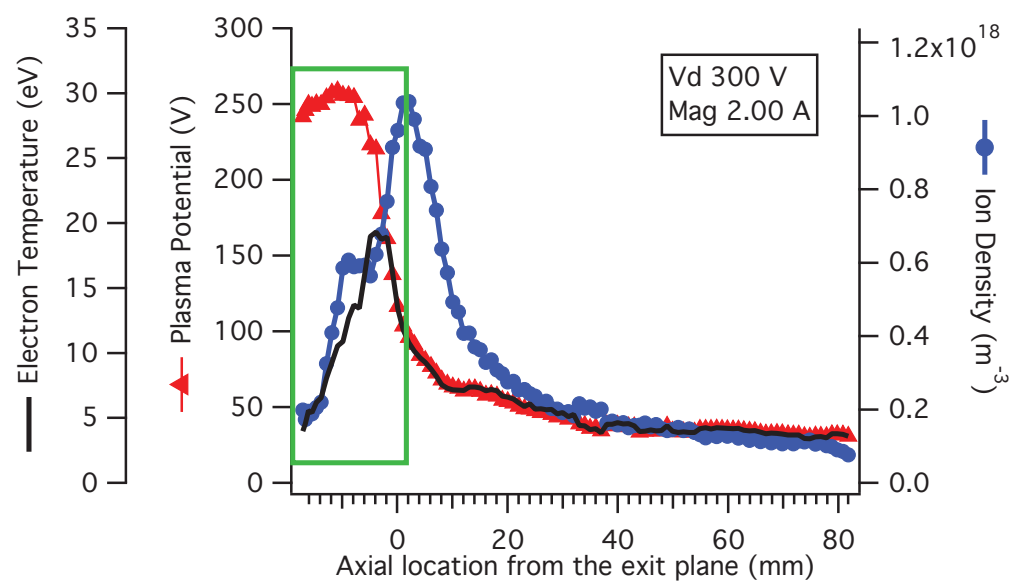

Figure 7.22: The green box represents the region over which the internal voltage utilization efficiency was calculated

Once calculated, the results of the iVUE are compared to the results of chap- 
ter 4 for the VUE in figures 7.23-7.29. The uncertainty in the plasma potential measurements carry through to be the dominant uncertainty in the iVUE calculations. Thus, in many cases trends in the iVUE are difficult to discern from the uncertainty. The low voltage results for krypton hold some of the strongest trends. The iVUE in figure 7.23 can clearly be seen to parallel both the $125 \mathrm{~V}$ and 100 $\mathrm{V}$ external RPA data by increasing with decreasing magnet current. Similarly, in the $150 \mathrm{~V}$ case, the iVUE is increasing with decreasing magnet current supporting the results from chapter 4 . In the higher voltages for krypton, the uncertainty masks any trends that may have been observed. In the case of xenon, the iVUE for the $125 \mathrm{~V}$ internal data peaks at the lowest magnet current just like the external VUE, however, that is within the uncertainty of the measurement. Similar to krypton, the high voltage trends in the iVUE are masked by the error bars for xenon.

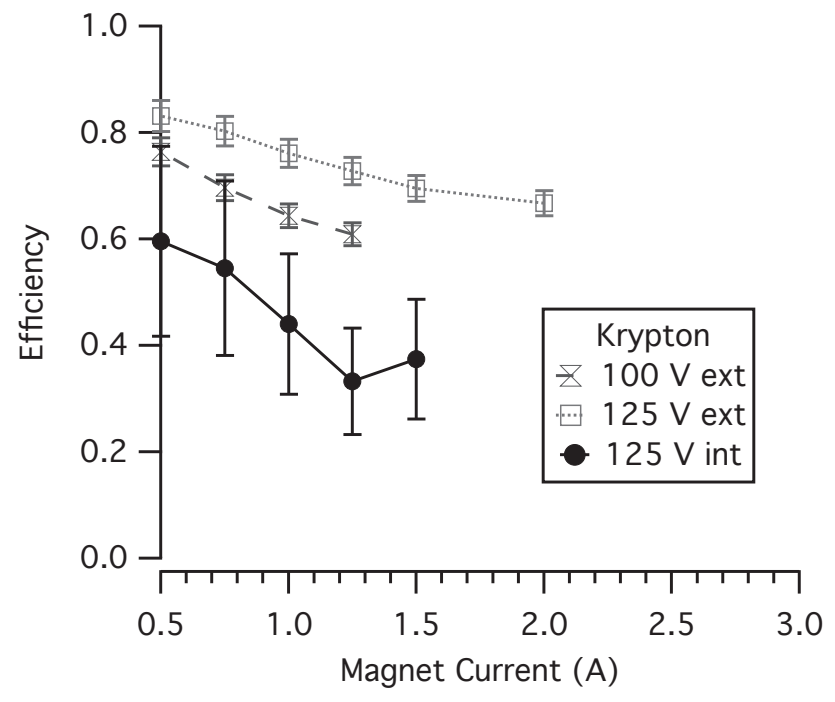

Figure 7.23: Internal voltage utilization efficiency as calculated from the HPS dataset compared to the voltage utilization efficiency calculated with the RPA probe in chapter 4 


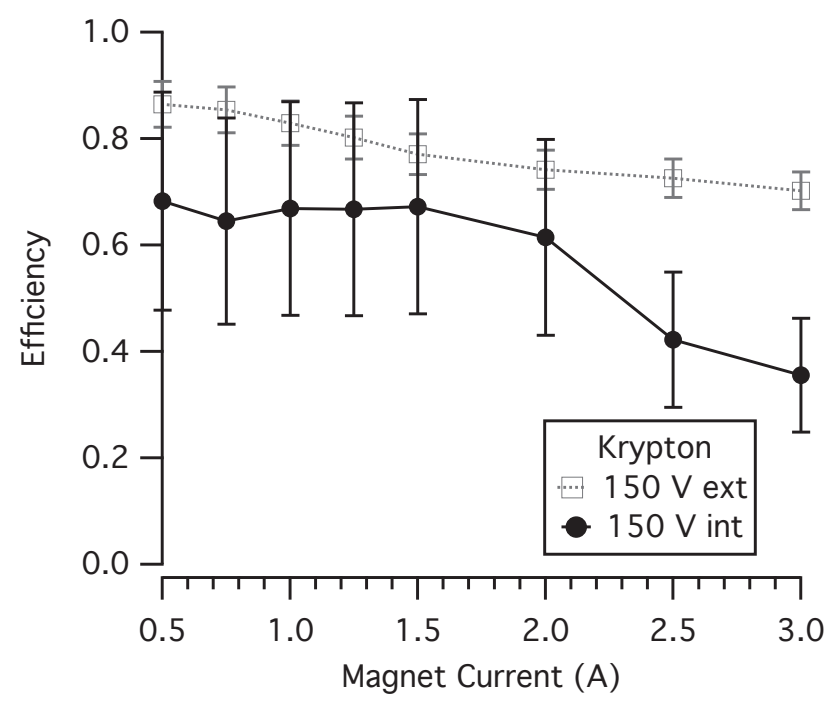

Figure 7.24: Internal voltage utilization efficiency as calculated from the HPS dataset compared to the voltage utilization efficiency calculated with the RPA probe in chapter 4

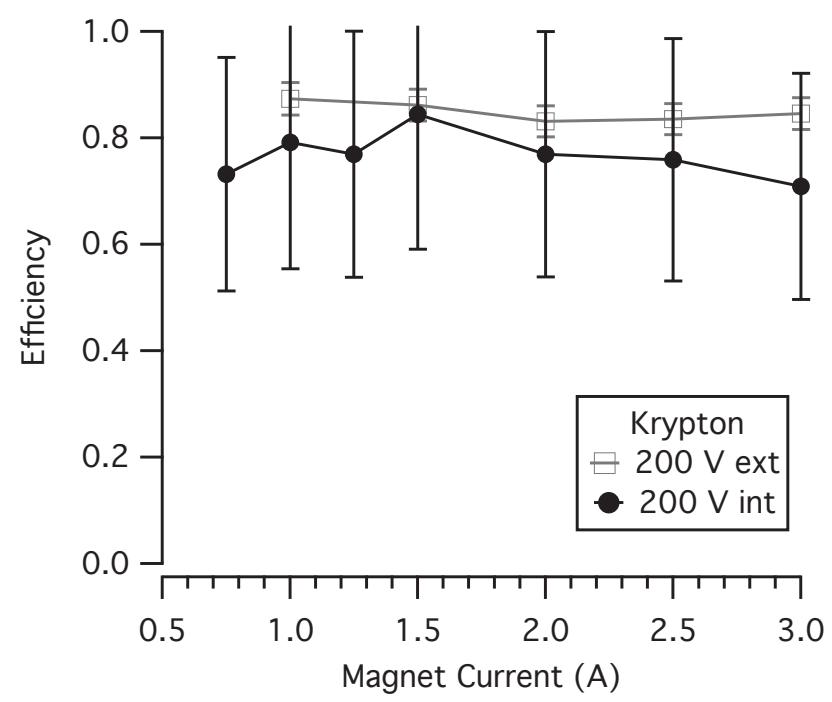

Figure 7.25: Internal voltage utilization efficiency as calculated from the HPS dataset compared to the voltage utilization efficiency calculated with the RPA probe in chapter 4 


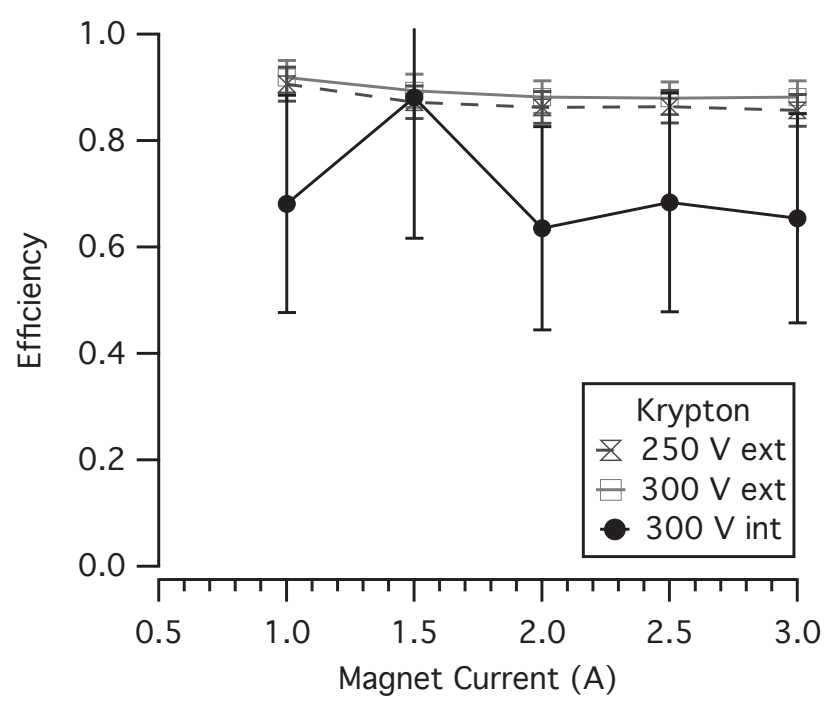

Figure 7.26: Internal voltage utilization efficiency as calculated from the HPS dataset compared to the voltage utilization efficiency calculated with the RPA probe in chapter 4

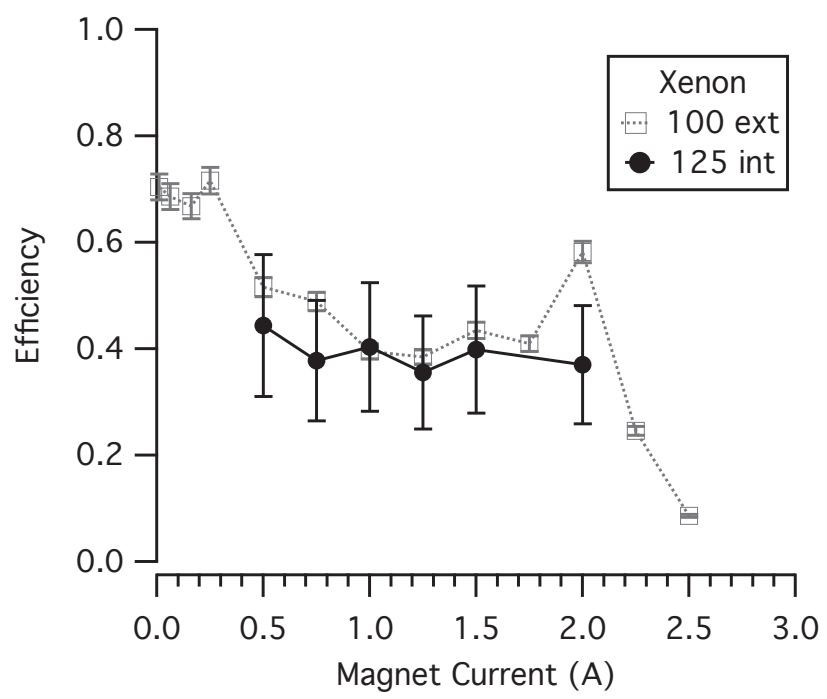

Figure 7.27: Internal voltage utilization efficiency as calculated from the HPS dataset compared to the voltage utilization efficiency calculated with the RPA probe in chapter 4 


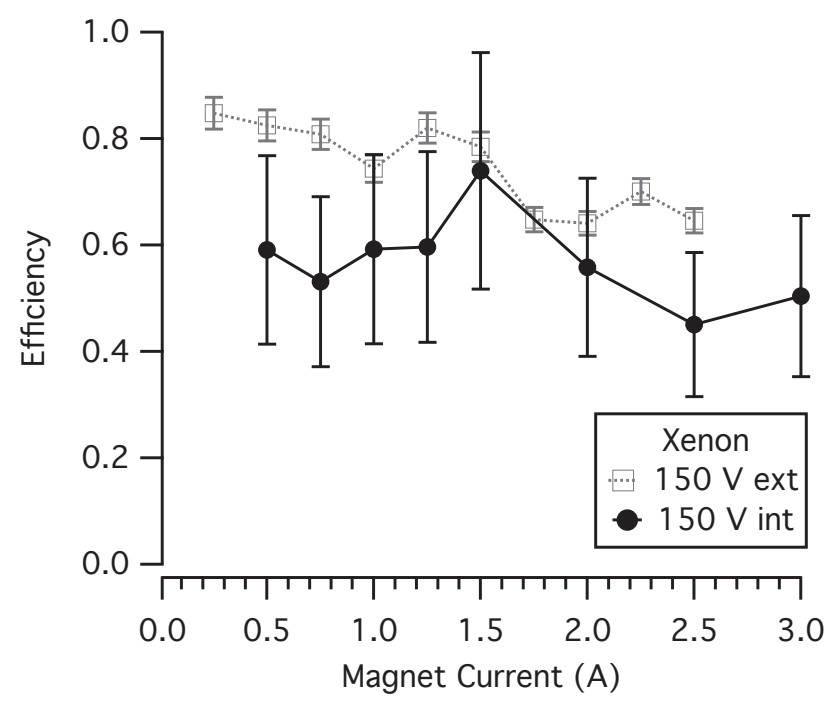

Figure 7.28: Internal voltage utilization efficiency as calculated from the HPS dataset compared to the voltage utilization efficiency calculated with the RPA probe in chapter 4

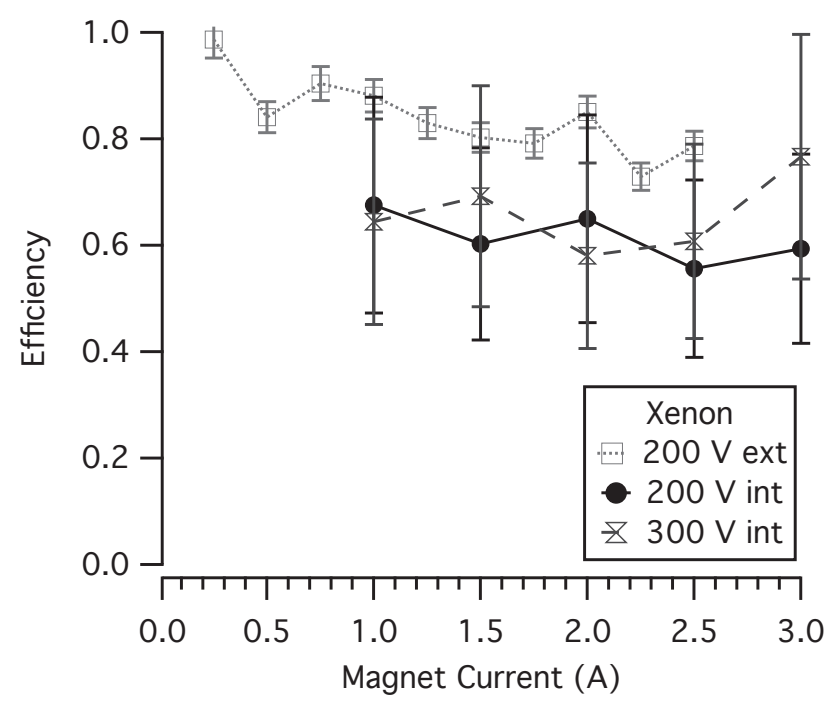

Figure 7.29: Internal voltage utilization efficiency as calculated from the HPS dataset compared to the voltage utilization efficiency calculated with the RPA probe in chapter 4 


\subsection{Conclusions}

Current efficiency is the dominant loss mechanism of low voltage operation of the BPT-2000 Hall thruster. Perhaps the more interesting finding was that the voltage utilization efficiency increases as a function of decreasing magnet current for all discharge voltages. This effect was was most pronounced for lower voltages.

The proportionality constant of magnetic field to discharge voltage, as defined in Ahedo's theoretical models, showed an increase in $p$ for reduced discharge voltage operation. The experimental results here did not agree, and showed a decrease in $p$ for low discharge voltage. This can attributed to the limitations of the models initial conditions. The model supposes that the bulk of the ion density will be upstream of the acceleration region. The internal data shows that the peak ion density can occur 10's of mm's downstream of the acceleration region for some operating conditions. Since the model does not have this degree of freedom, it cannot solve for the appropriate optimized magnet circuit.

The high-speed probe test for this work is a first of its kind for low voltage thrusters. Many internal probe studies for high discharge voltage work exist in the community and have led design modifications to improve overall efficiency. Among the internal probe results, it was confirmed that the ionization zone moves upstream with respect to the acceleration region with decreased magnet current for low voltages. The natural result of this is an increase in voltage utilization efficiency, which is supported by the results of the external probe data of chapter 4. Decreasing the magnet current was also shown to increase the peak ion density and shift the entire ion density profile further upstream for low voltage operation. At higher voltages, the magnet current had a much smaller effect on both the location of the ionization and acceleration zones. No change in the fraction of potential drop in the acceleration region was determined as a function of magnet current for any voltage/propellant type.

The axial shift in the ionization and acceleration locations as a function of magnet current for low voltage operation was also shown to be a location shift to lower magnetic field values when compared to the higher discharge voltages at the same magnet currents. This indicates that it may be necessary for the ionization and acceleration regions to be located at lower magnetic field strengths to sustain ionization processes for low voltages. This phenomenon may be explored in detailed future work by altering the magnetic field structure, shape, number of coils etc., with the aim to increase the voltage utilization while reducing anode current. 


\section{Bibliography}

[1] Brown, D. L., Investigation of Low Discharge Voltage Hall Thruster Characteristics and Evaluation of Loss Mechanisms, Ph.D. thesis, University of Michigan, June 2009.

[2] Larson, C. W., Brown, D. L., and Hargus, W. A., "Thrust Efficiency, Energy Efficiency, and the Role of the VDF in Hall Thruster Performance Analysis," 43rd AIAA/ASME/SAE/ASEE Joint Propulsion Conference and Exhibit, Vol. AIAA 2007-5270, Cincinnati, Ohio, July 8-11 2007.

[3] Hofer, R. R. and Gallimore, A. D., "Efficiency Analysis of a High-Specific Impulse Hall Thruster," 40th AIAA/ASME/SAE/ASEE Joint Propulsion Conference \& Exhibit, Vol. AIAA-2004-3602, Ft. Lauderdale, Florida, July 11-14 2004.

[4] Belan, N. V., Kim, V. P., Oranskiy, A. I., and Tikhonov, V. B., "Stationary Plasma Engines - Translation of "Statsionarnyye plasmennyye dvigateli"," UUSR State Committee on Public Education, N. Ye. Zhukovskiy Kharkov Order of Lenin Aviation Institute, 1989, pp. 1-49.

[5] E. Ahedo, D. E., "Influence of design and operation parameters on Hall thruster performances," Journal of Applied Physics, Vol. 96(2), No. 2, 2004, pp. 10.

[6] Kim, V., "Main Physical Features and Processes Determining the Performace of Stationary Plasma Thrusters," Journal of Propulsion and Power, Vol. 14, No. 5, 1998, pp. 736-743.

[7] Manzella, D., Jacobson, D., and Jankovsky, R. F. K., "High Voltage SPT Performance," 37th Joint Propulsion Conference and Exhibit, Salt Lake City, Utah, July 8-11 2001.

[8] Sommerville, J. D., Hall-Effect Thruster-Cathode Coupling: The Effect of Cathode Position and Magnetic Field Topology, Ph.D. thesis, Michigan Technological University, 2009. 
[9] Griffiths, D. J., Introduction to Electrodynamics (3rd Edition), Benjamin Cummings, 3rd ed., January 1999.

[10] Reid, B. M. and Gallimore, A. D., "Langmuir Probe Measurements in the Discharge Channel of a 6-kW Hall Thruster," 44th AIAA/ASME/SAE/ASEE Joint Propulsion Conference and Exhibit, Vol. AIAA-2008-4920, Hartford, CT, July 20-23 2008.

[11] Reid, B. M. and Gallimore, A. D., "Plasma Potential Measurements in the Discharge Channel of a 6-kW Hall Thruster," 44th AIAA/AS$M E / S A E / A S E E$ Joint Propulsion Conference and Exhibit, Vol. AIAA-20085185, Hartford, CT, July 20-23 2008.

[12] Chen, F. F., Plasma Diagnostic Techniques, Academic Press, New York, NY, 1965.

[13] Hutchinson, I. H., Principles of Plasma Diagnostics, Cambridge University Press, 1987.

[14] Linnell, J. A. and Gallimore, A. D., "Internal Langmuir Probe Mapping of a Hall Thruster with Xenon and Krypton Prop," Vol. AIAA-2006-4470, Sacramento, California, 9-12 July 2006. 


\section{Appendix A}

\section{HPS Data}

\section{A.1 Xenon}

This is the complete data set results of the high-speed probe tests for xenon. The experimental design can be found in chapter 5 and summary of the results is found in chapter 6 For each graph a textbox lists the discharge voltage and magnet current corresponding to the data set for the recorded traces.

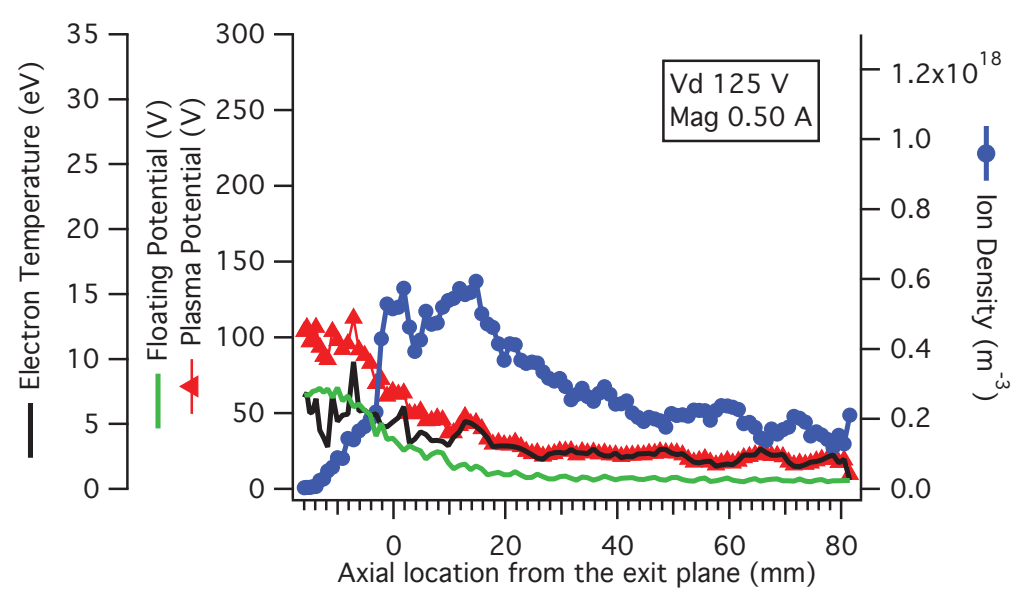

Figure A.1: High-speed probe results for Xenon 


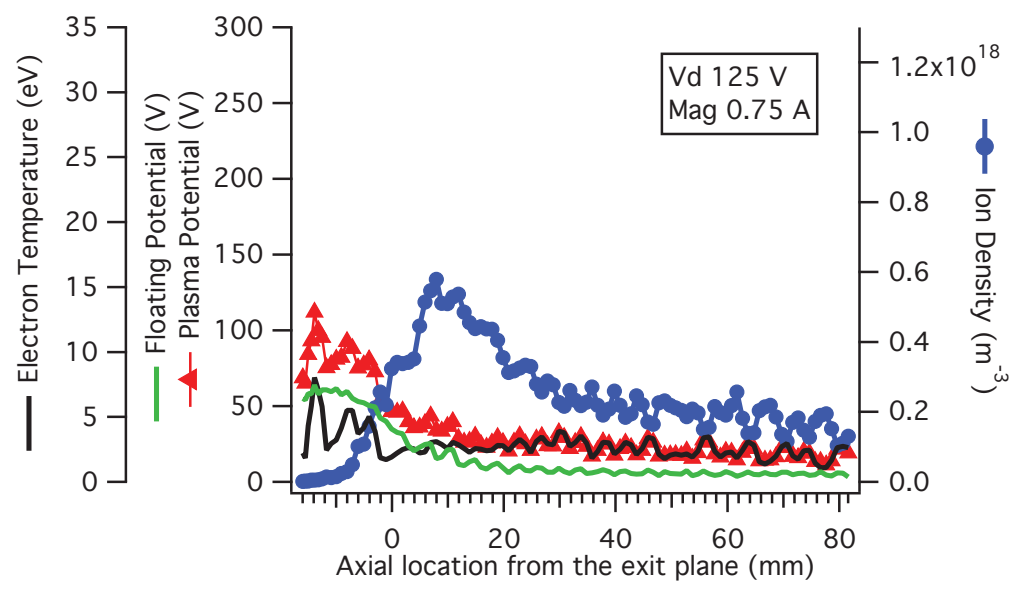

Figure A.2: High-speed probe results for Xenon

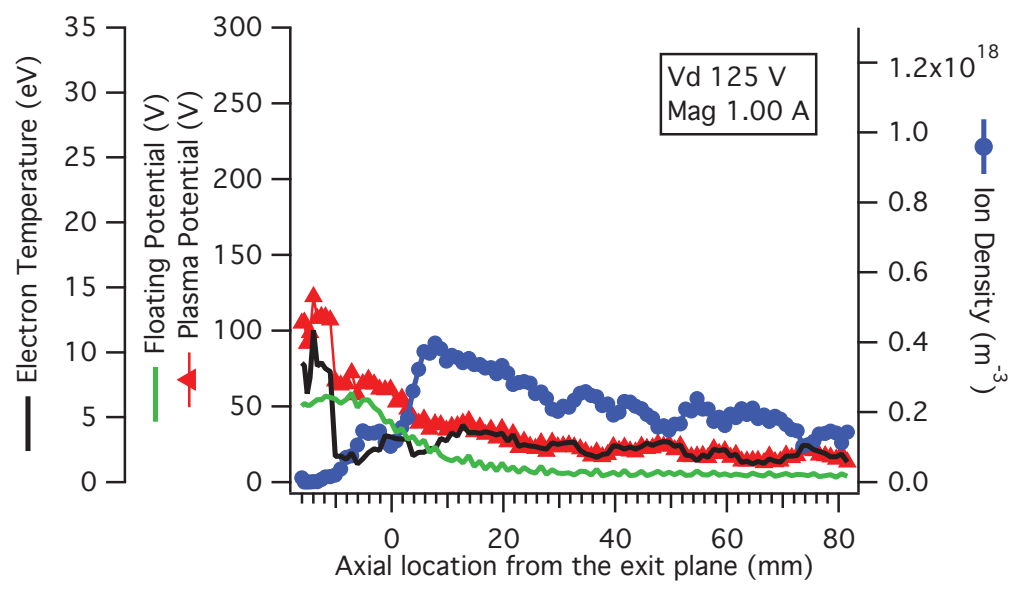

Figure A.3: High-speed probe results for Xenon 


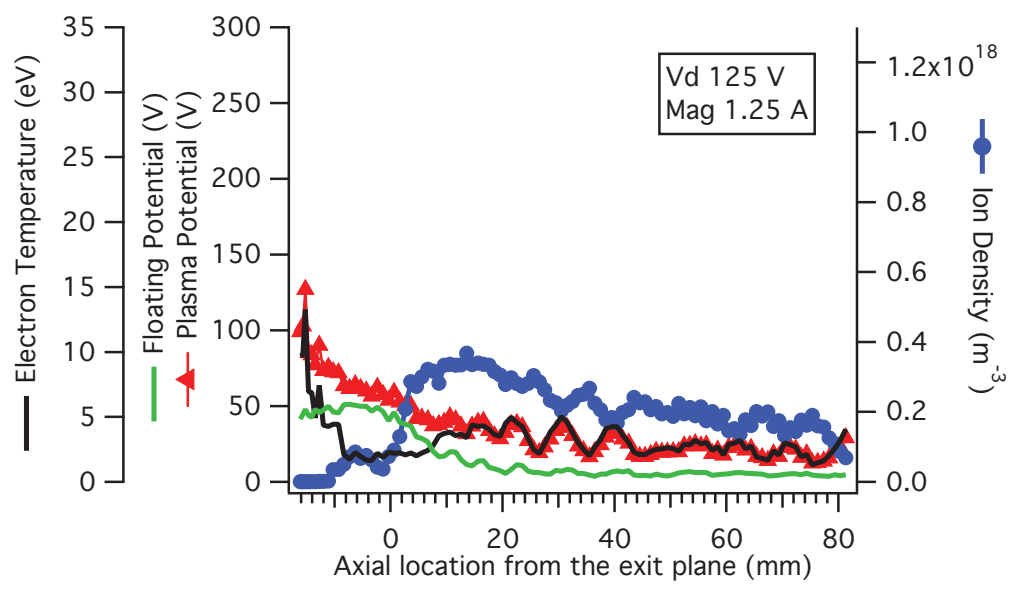

Figure A.4: High-speed probe results for Xenon

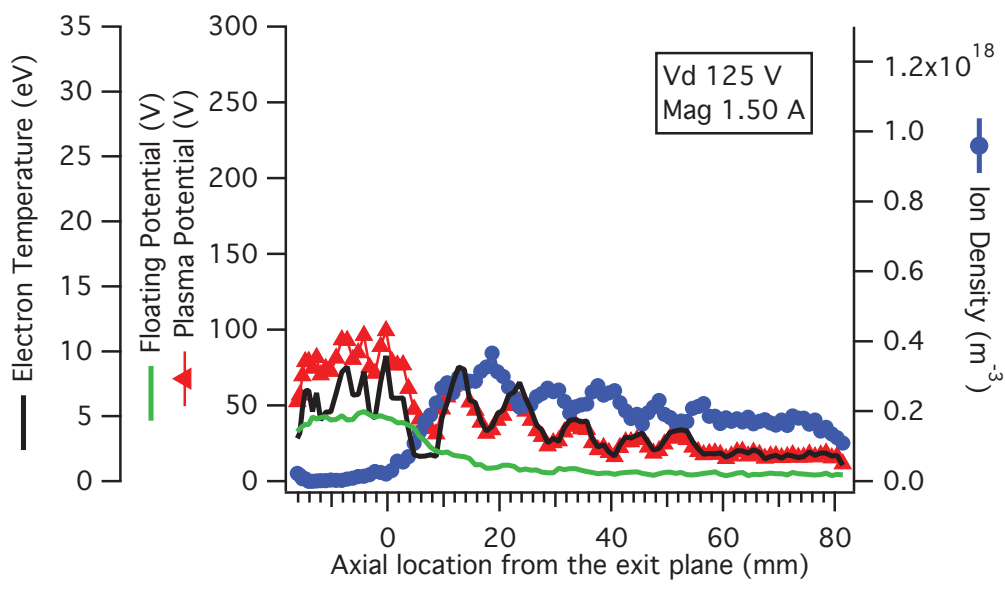

Figure A.5: High-speed probe results for Xenon 


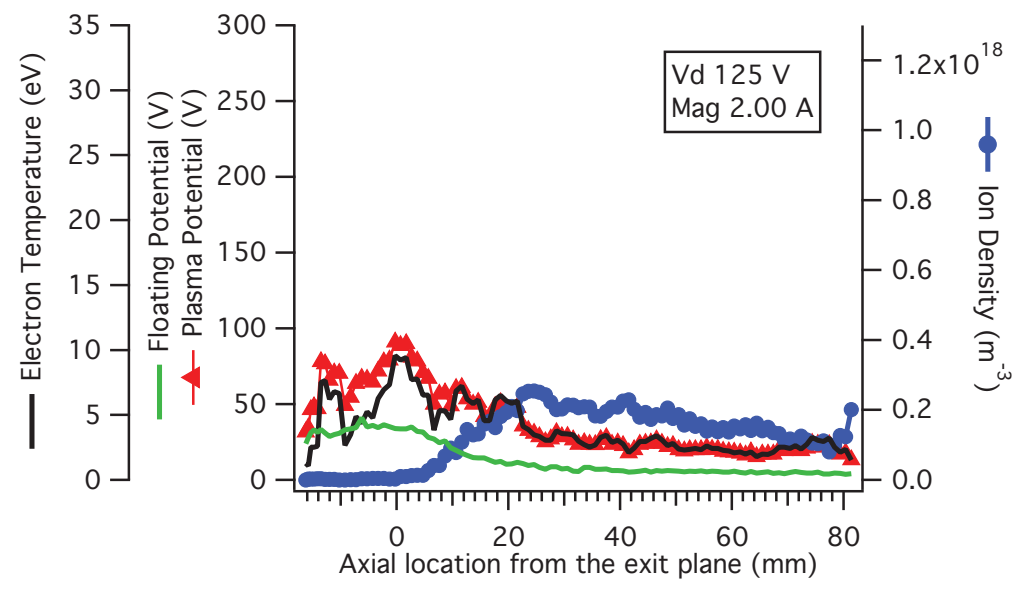

Figure A.6: High-speed probe results for Xenon

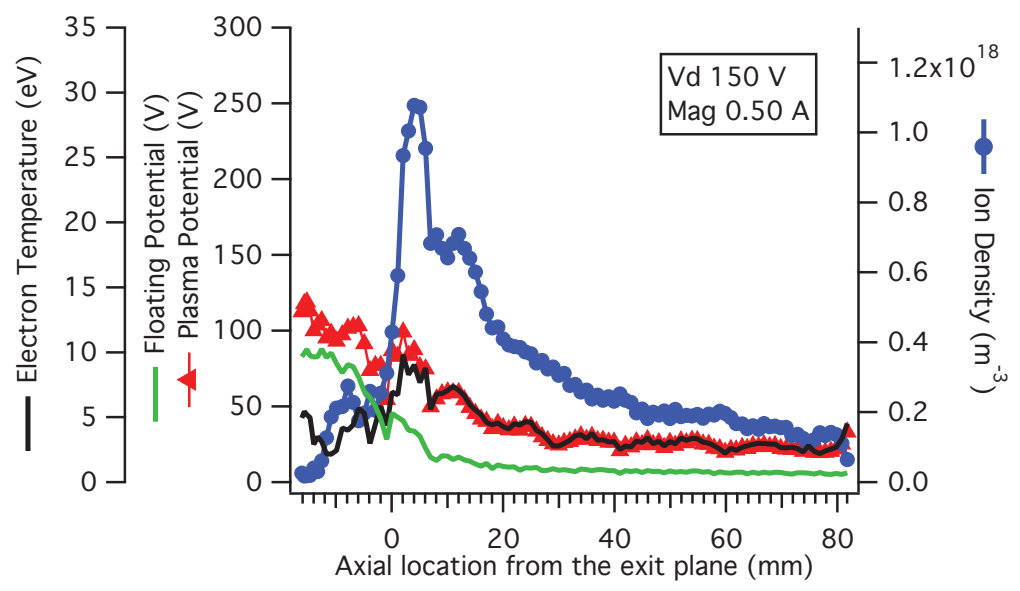

Figure A.7: High-speed probe results for Xenon 


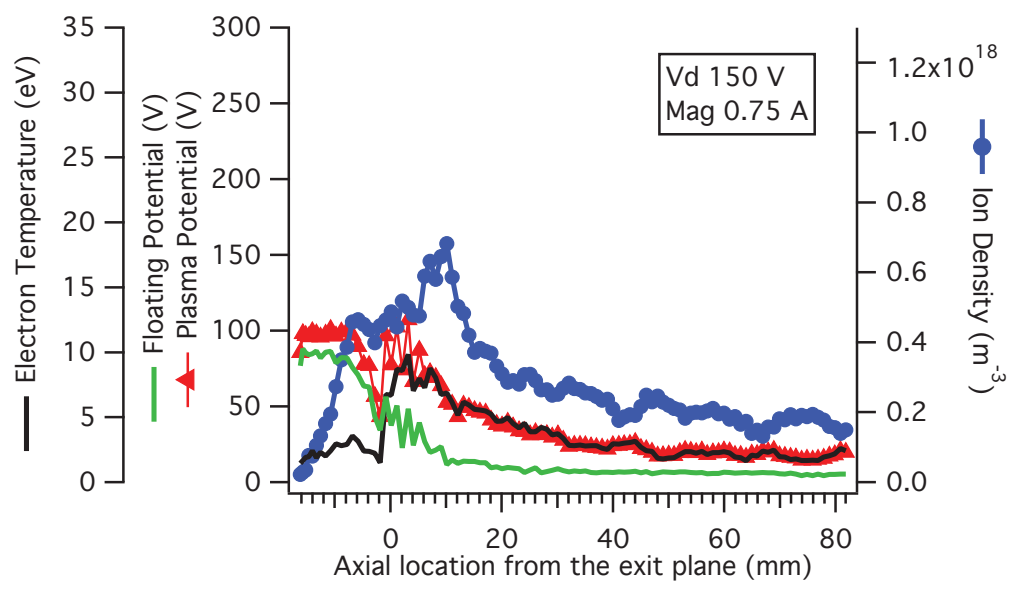

Figure A.8: High-speed probe results for Xenon

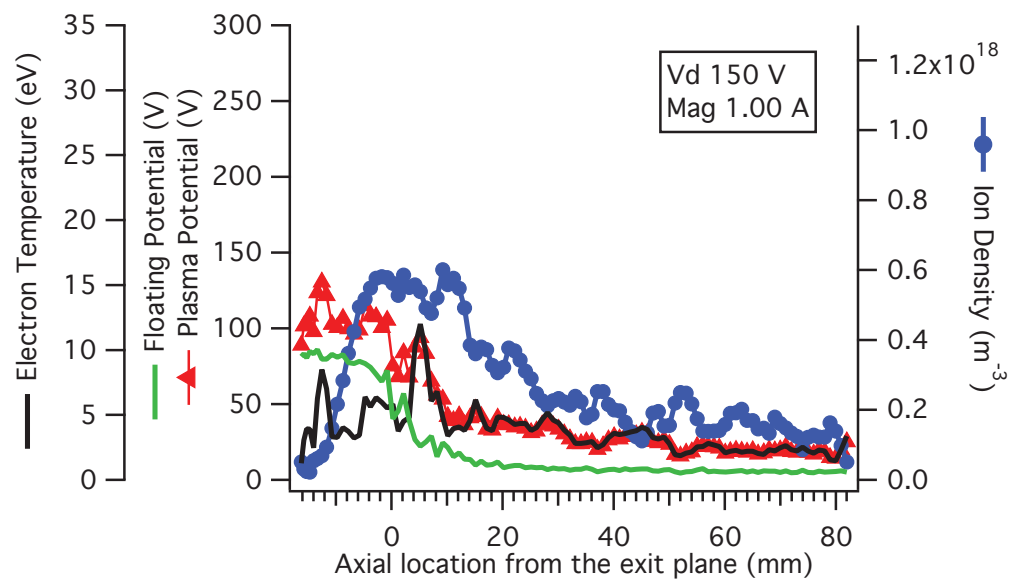

Figure A.9: High-speed probe results for Xenon 


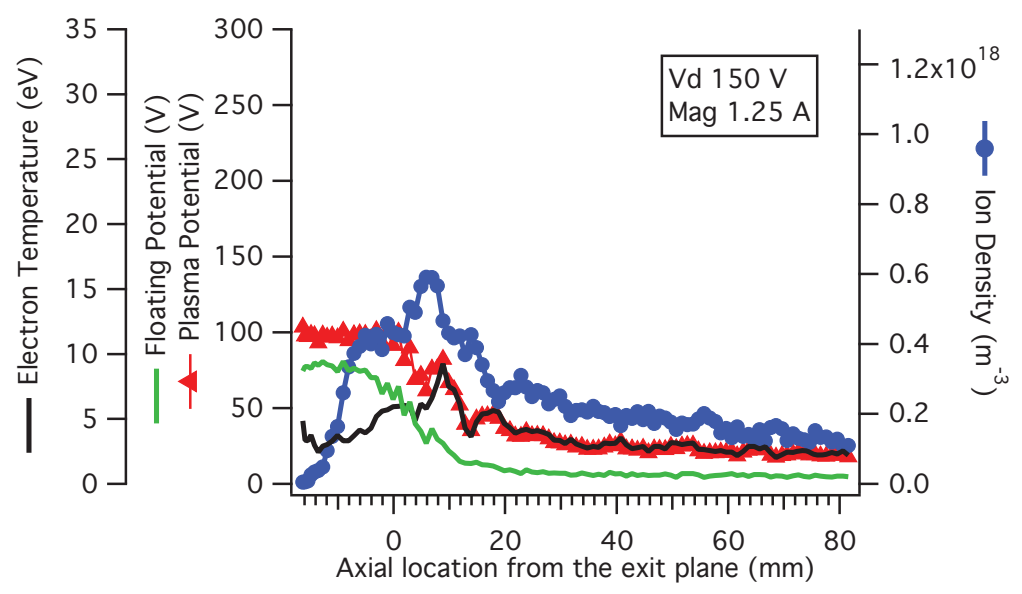

Figure A.10: High-speed probe results for Xenon

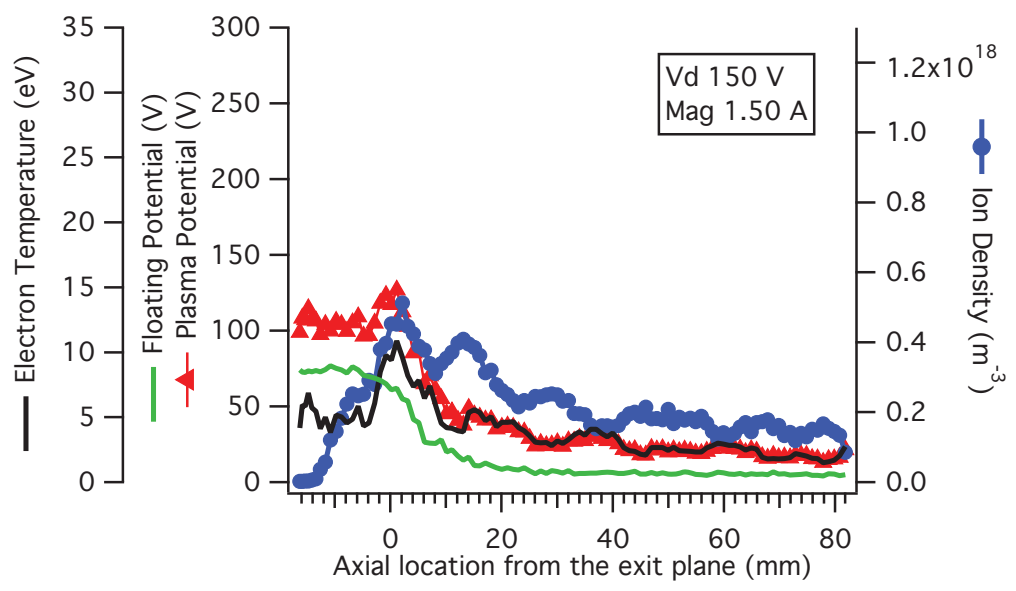

Figure A.11: High-speed probe results for Xenon 


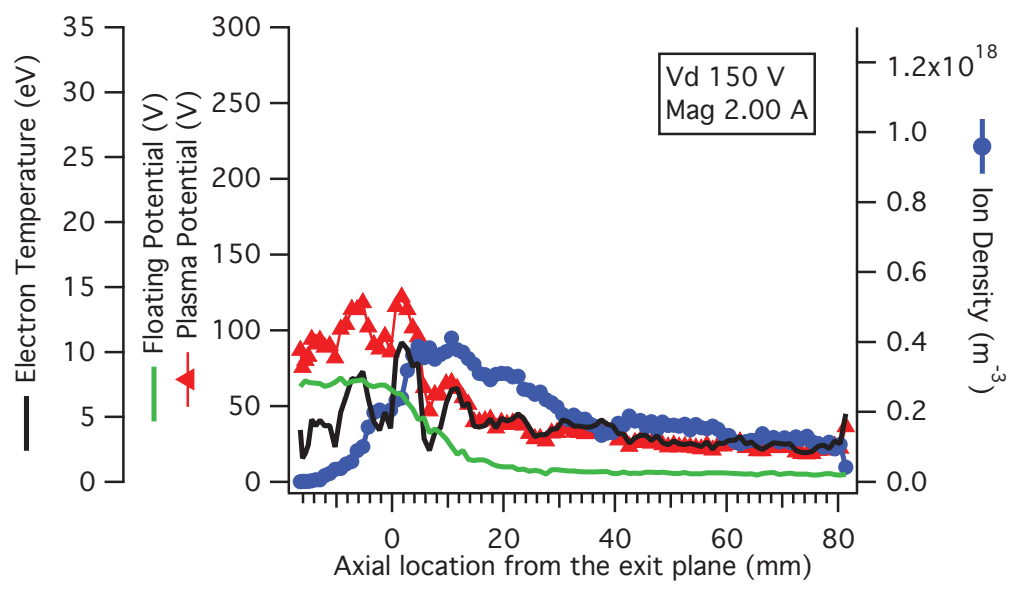

Figure A.12: High-speed probe results for Xenon

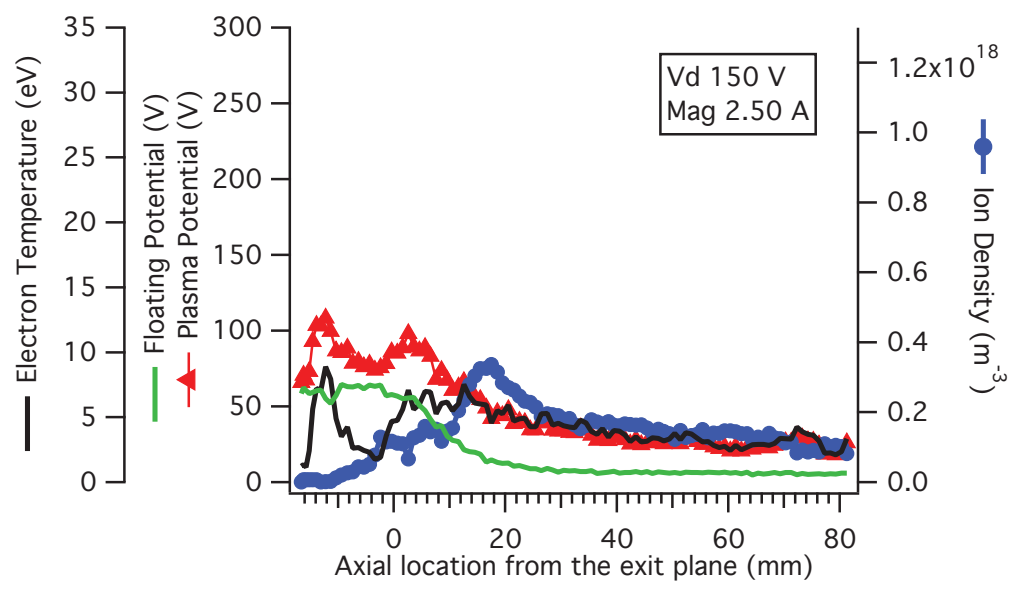

Figure A.13: High-speed probe results for Xenon 


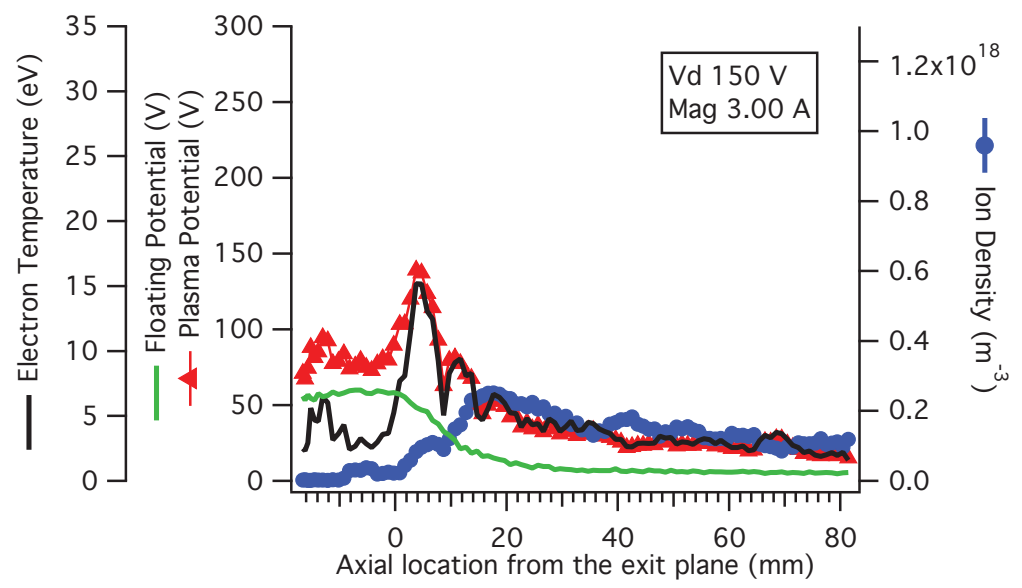

Figure A.14: High-speed probe results for Xenon

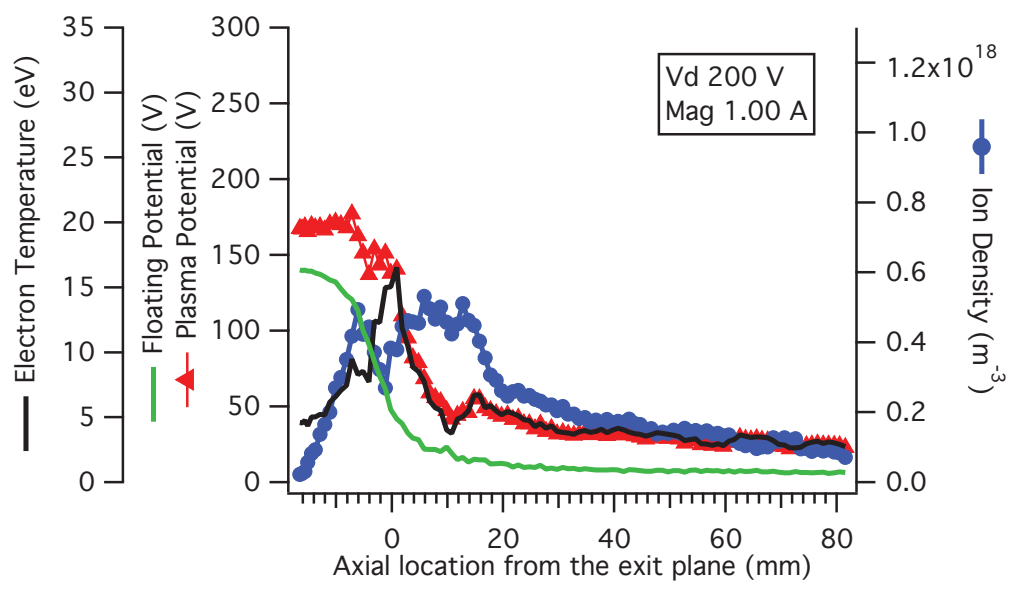

Figure A.15: High-speed probe results for Xenon 


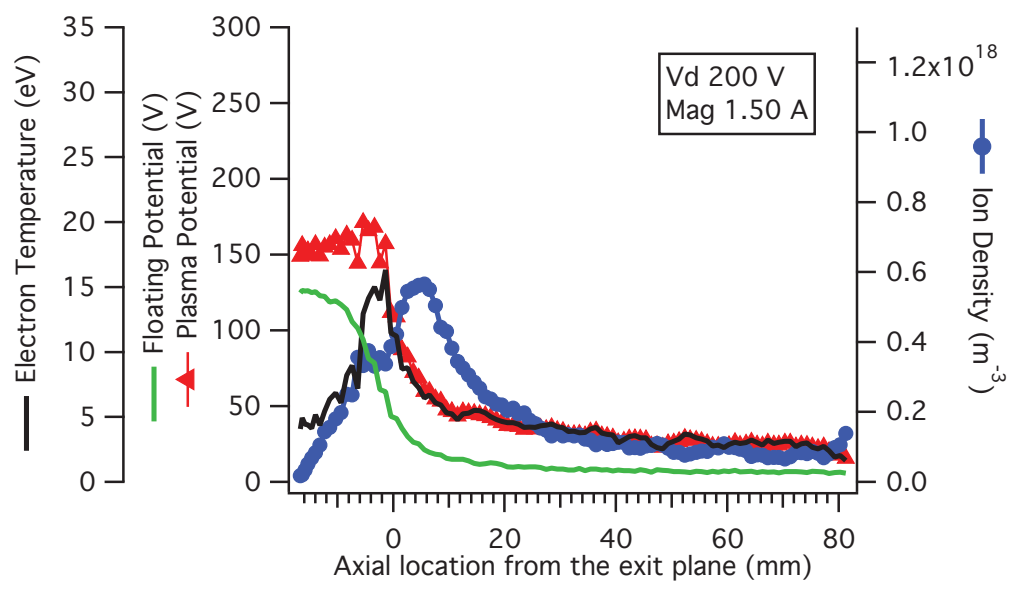

Figure A.16: High-speed probe results for Xenon

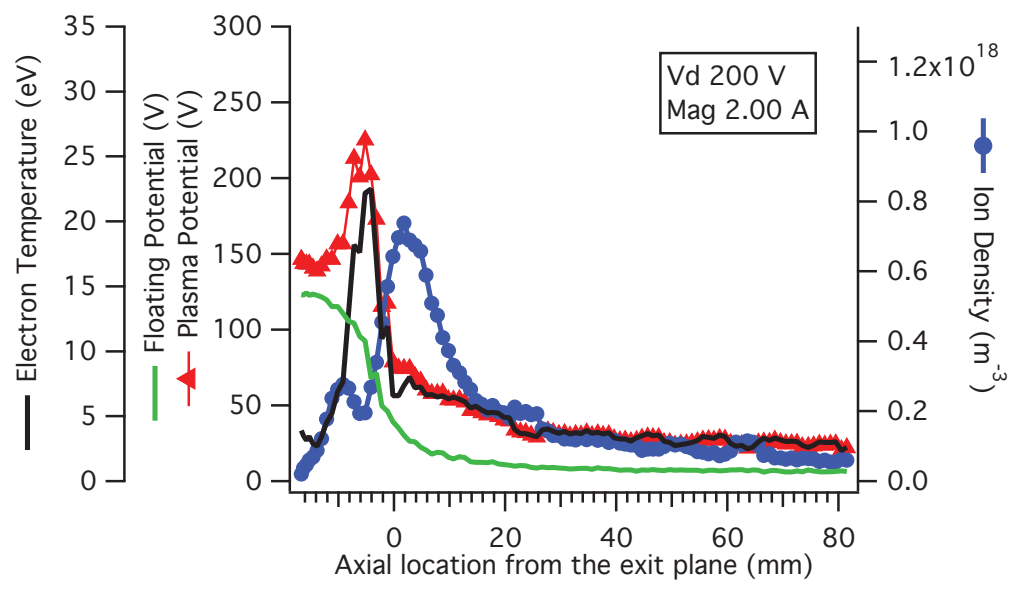

Figure A.17: High-speed probe results for Xenon 


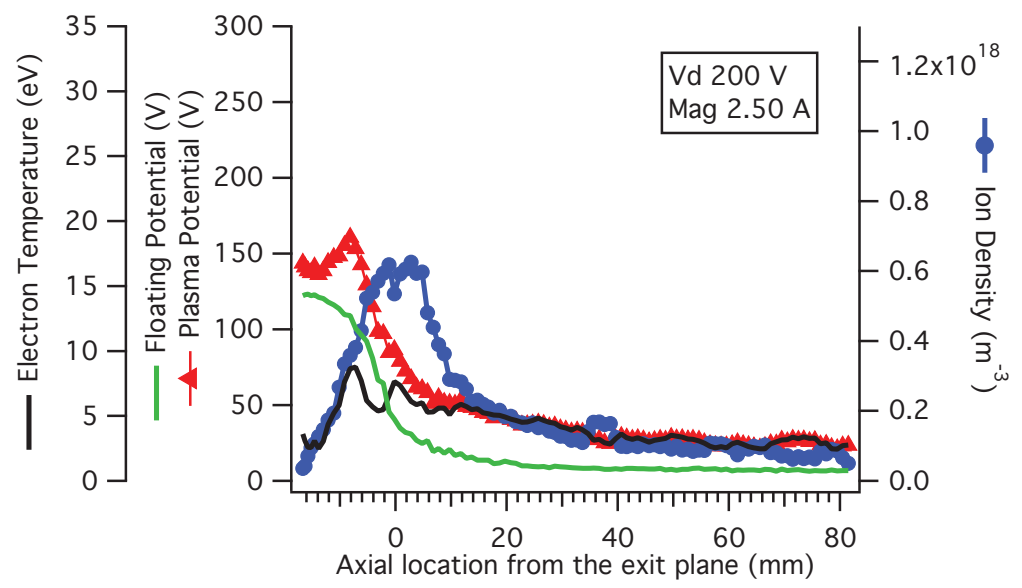

Figure A.18: High-speed probe results for Xenon

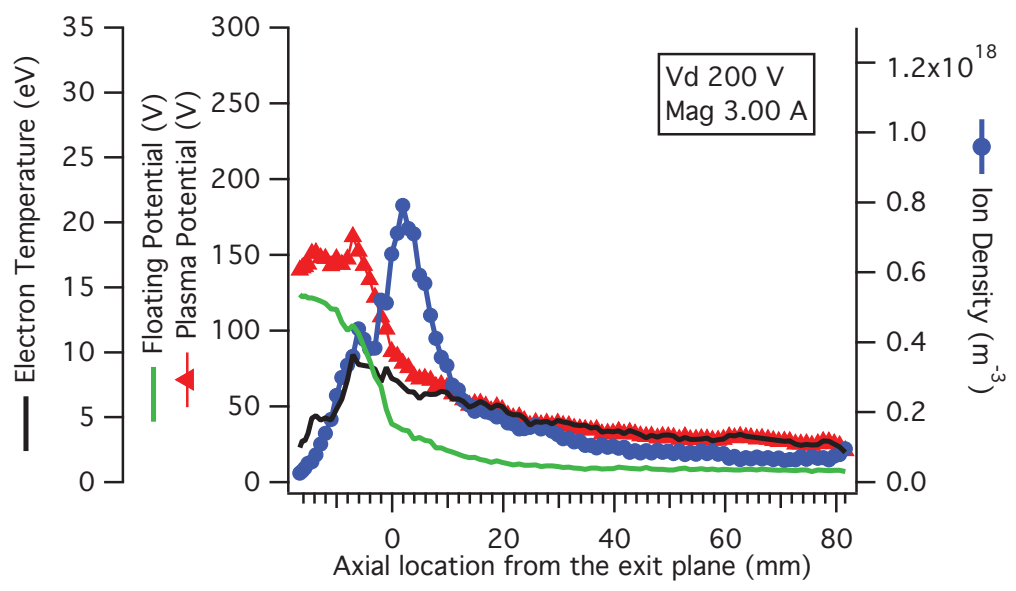

Figure A.19: High-speed probe results for Xenon 


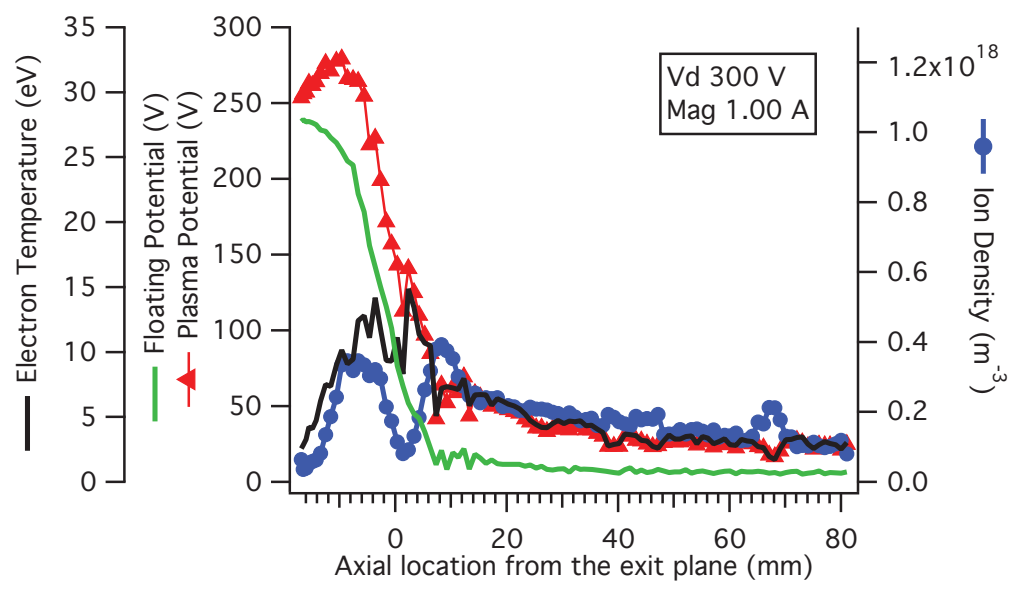

Figure A.20: High-speed probe results for Xenon

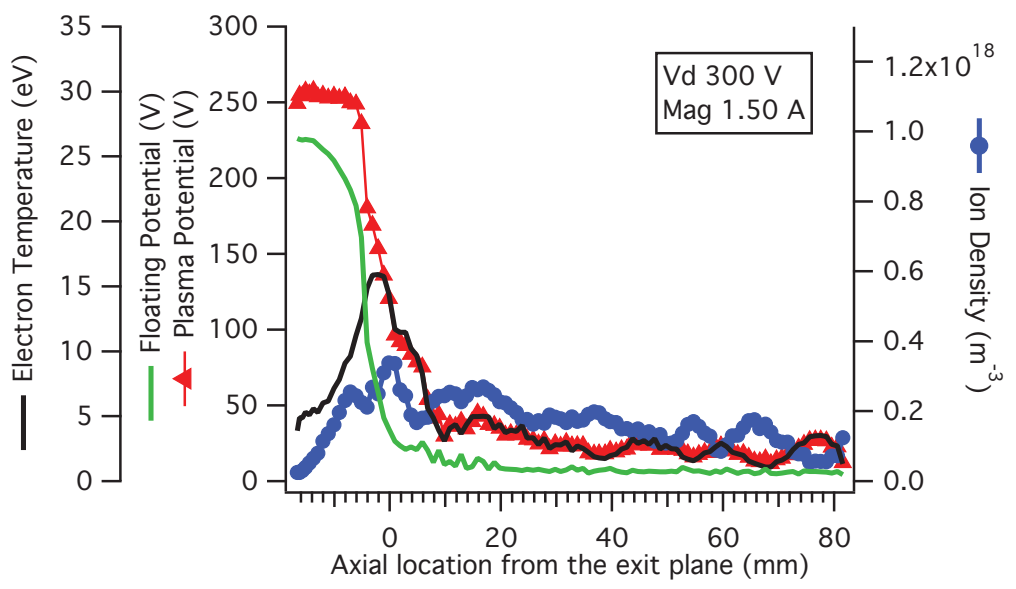

Figure A.21: High-speed probe results for Xenon 


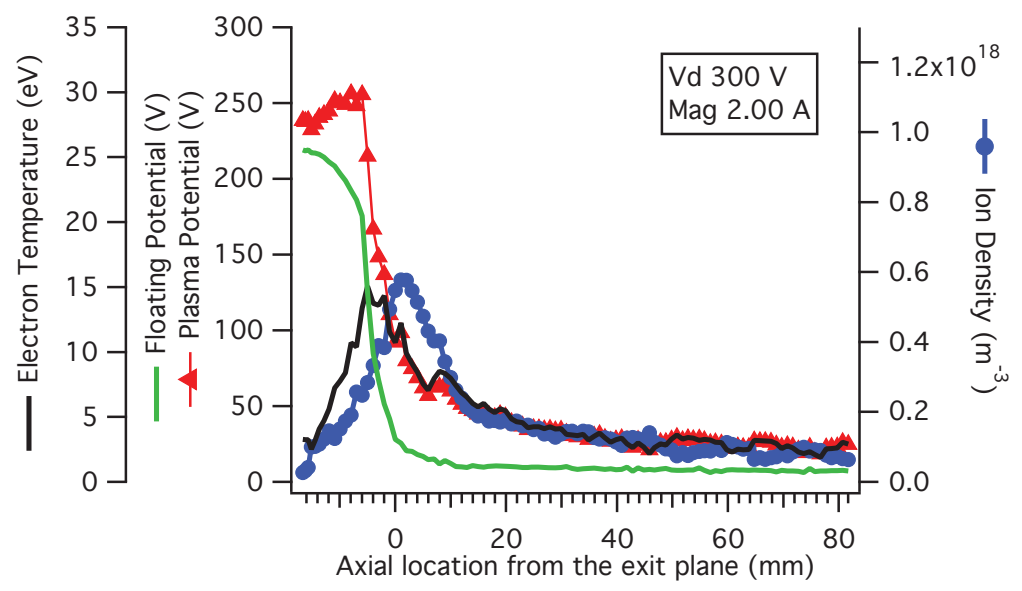

Figure A.22: High-speed probe results for Xenon

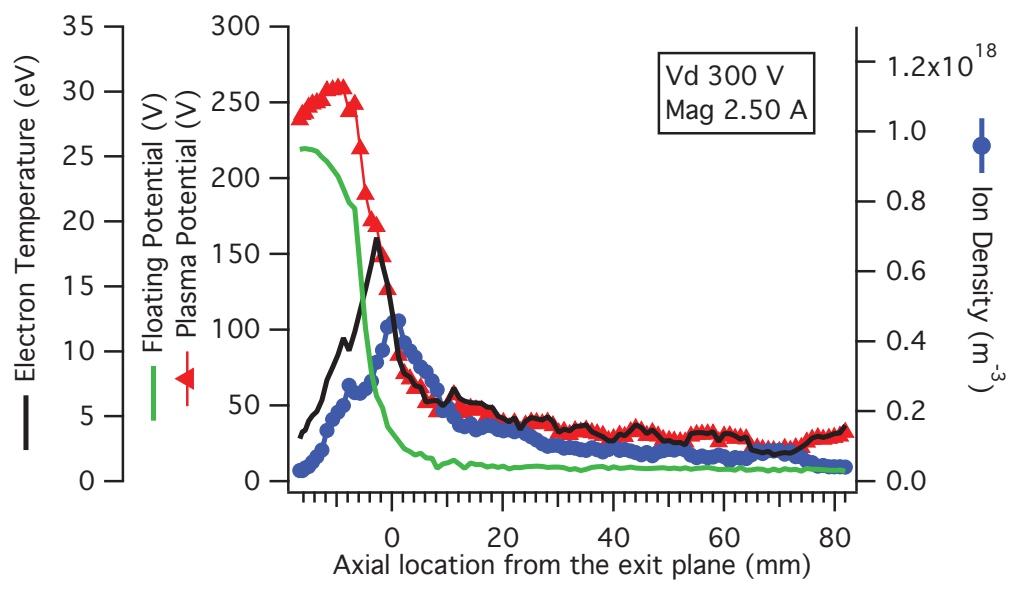

Figure A.23: High-speed probe results for Xenon 


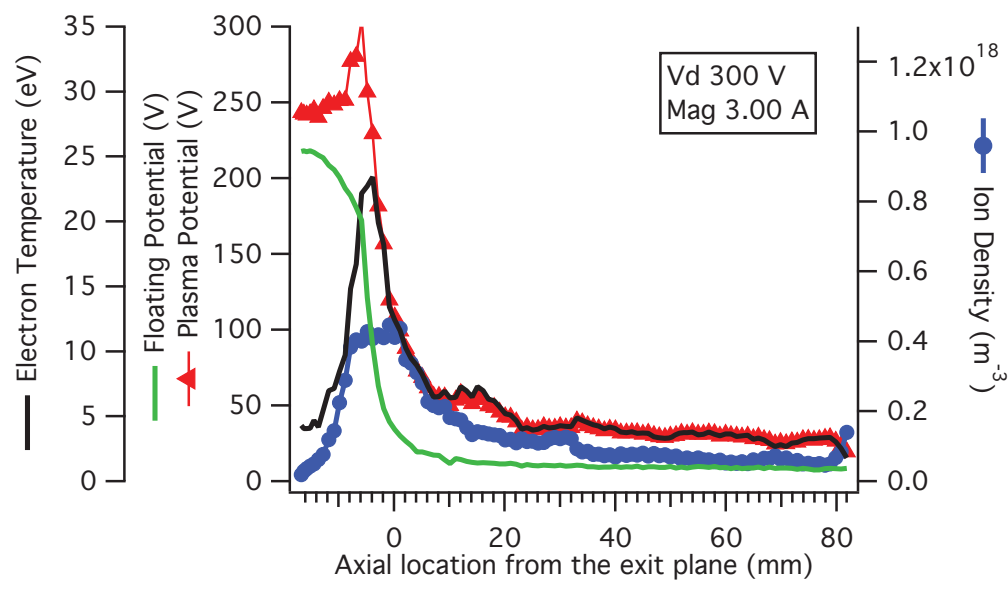

Figure A.24: High-speed probe results for Xenon 


\section{A.2 Krypton}

This is the complete data set results of the high-speed probe tests for krypton. The experimental design can be found in chapter 5 and summary of the results is found in chapter 6 For each graph a textbox lists the discharge voltage and magnet current corresponding to the data set for the recorded traces.

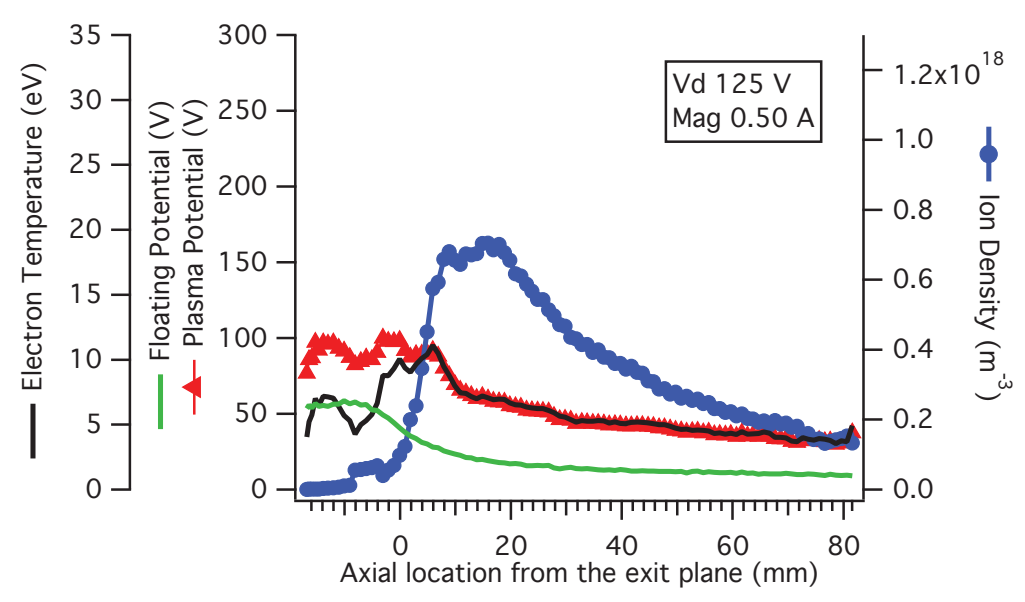

Figure A.25: High-speed probe results for Krypton

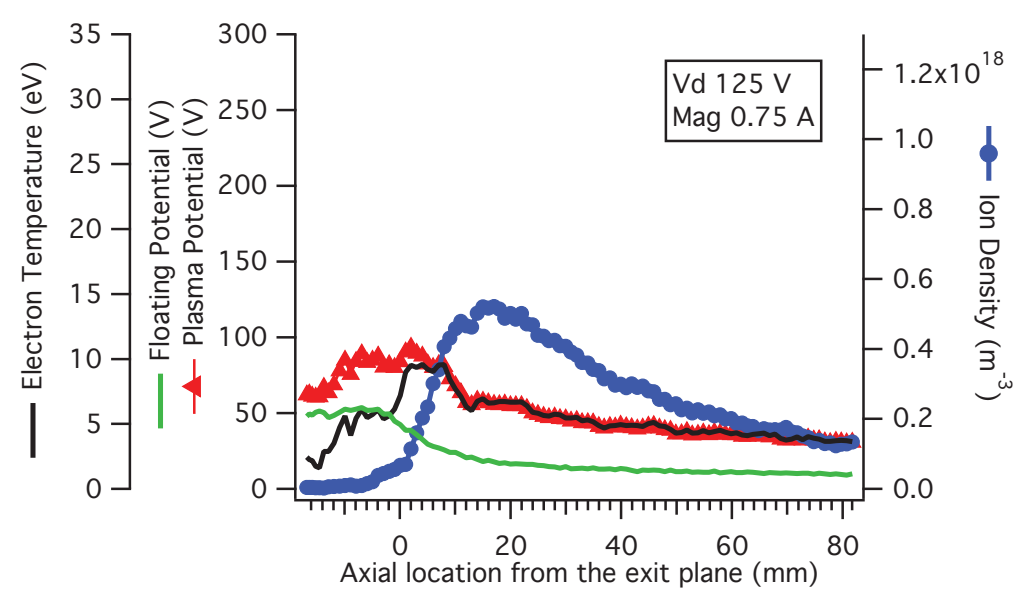

Figure A.26: High-speed probe results for Krypton 


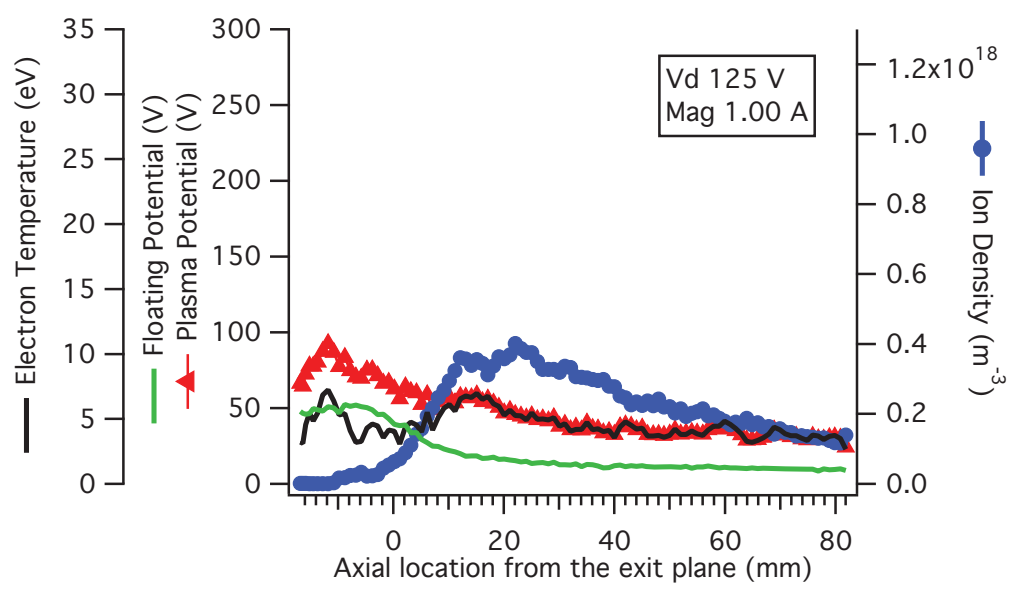

Figure A.27: High-speed probe results for Krypton

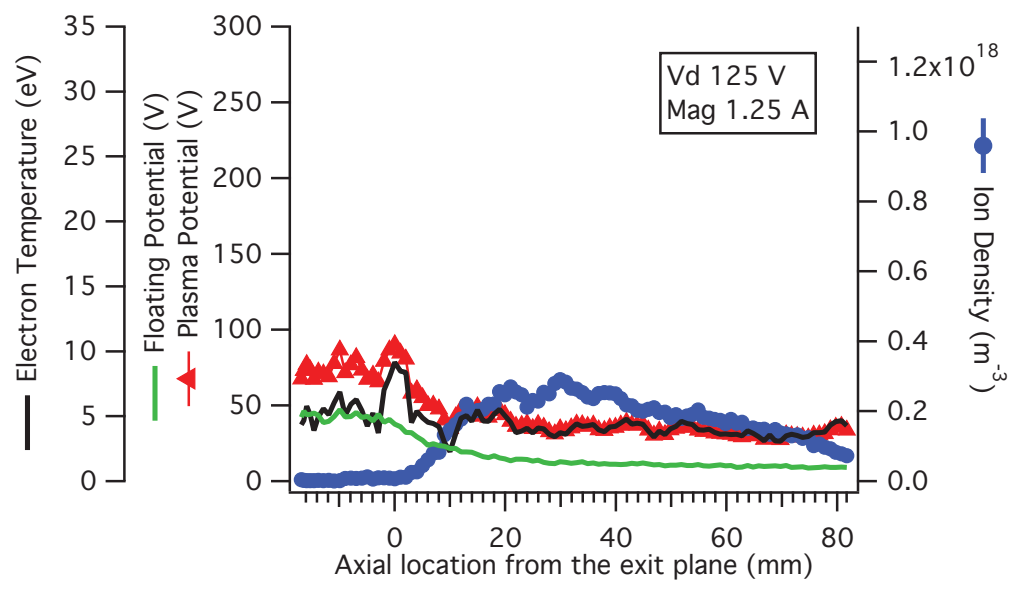

Figure A.28: High-speed probe results for Krypton 


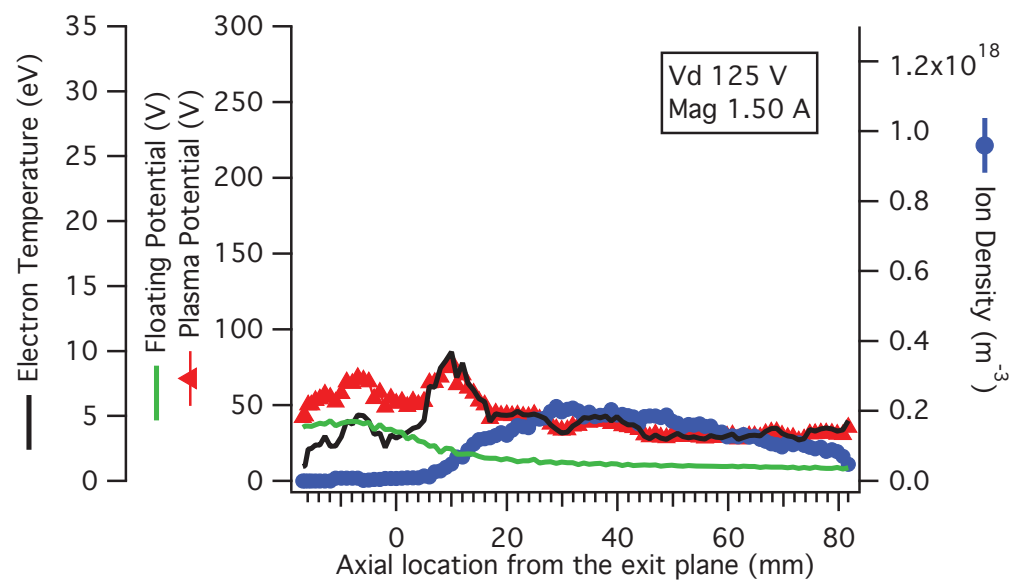

Figure A.29: High-speed probe results for Krypton

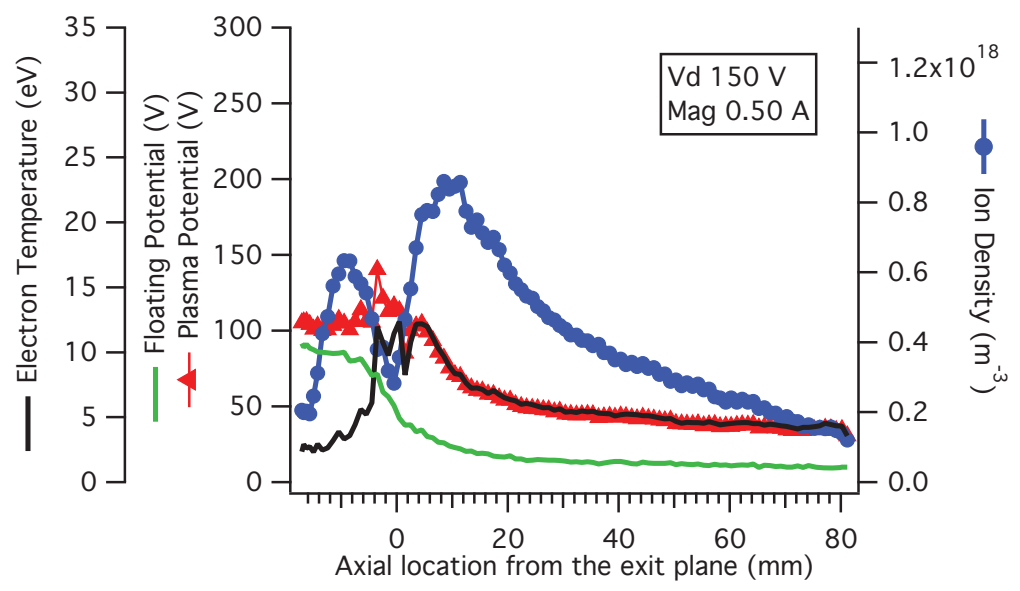

Figure A.30: High-speed probe results for Krypton 


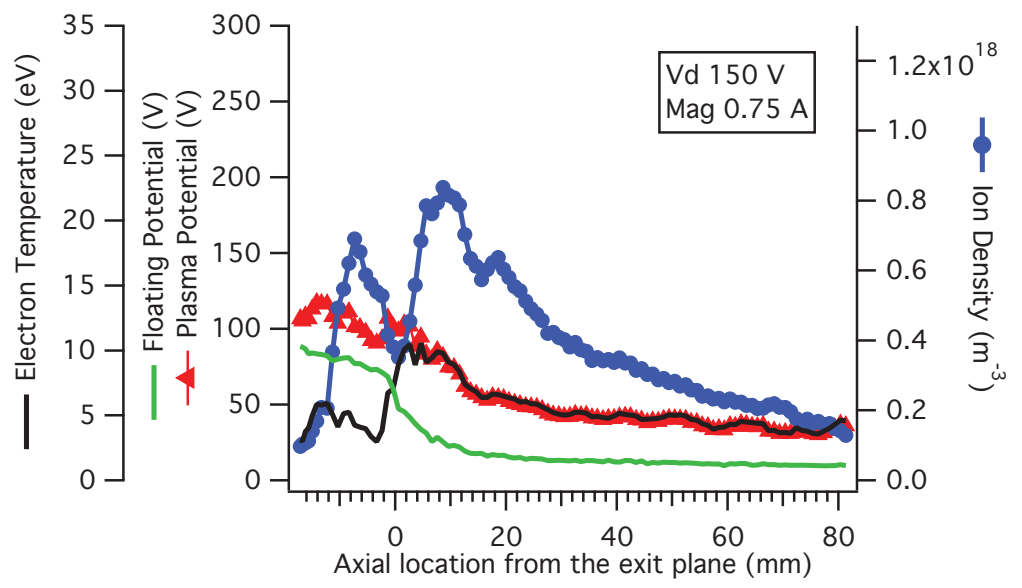

Figure A.31: High-speed probe results for Krypton

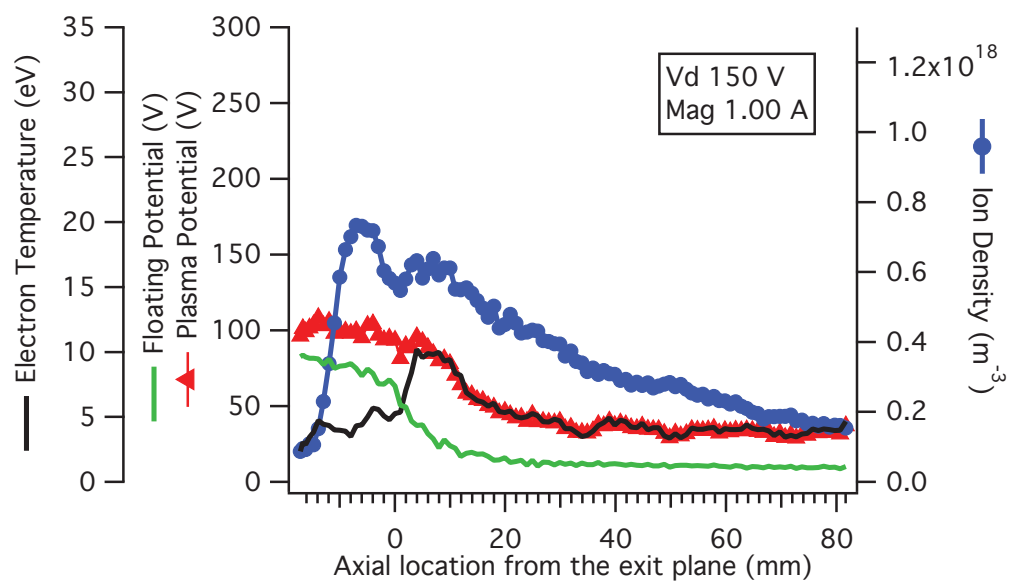

Figure A.32: High-speed probe results for Krypton 


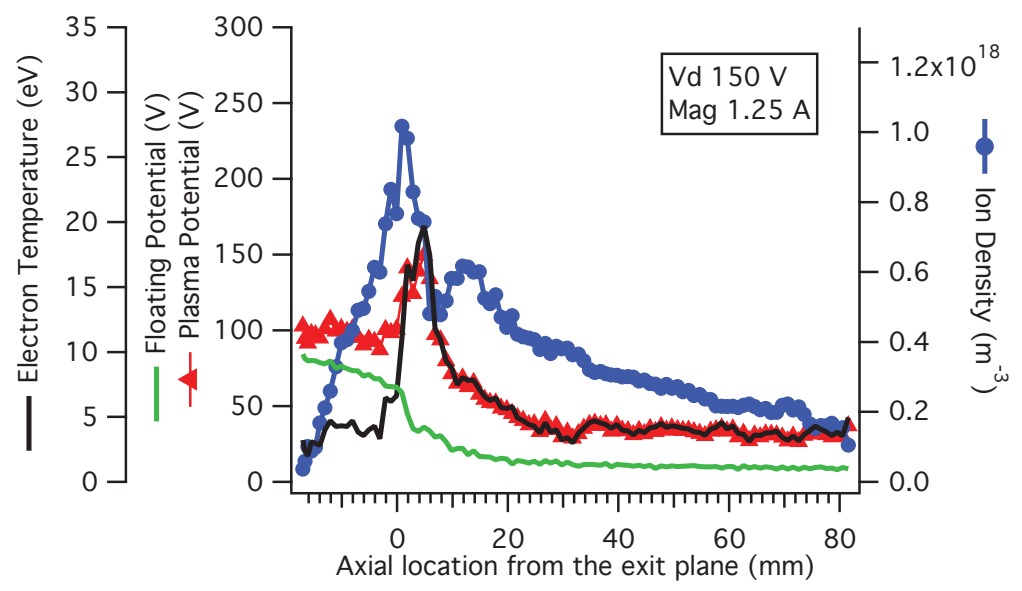

Figure A.33: High-speed probe results for Krypton

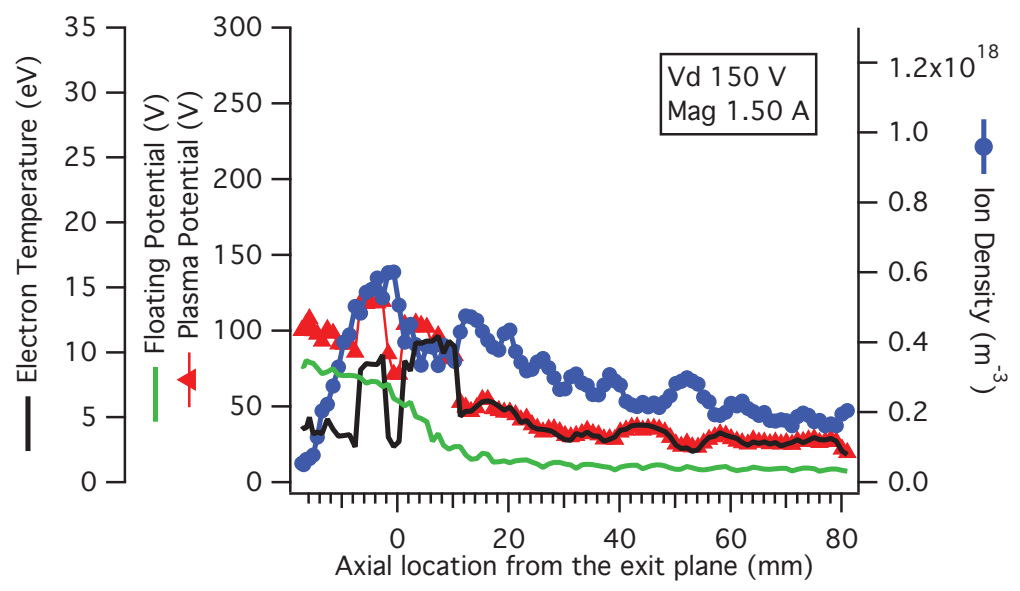

Figure A.34: High-speed probe results for Krypton 


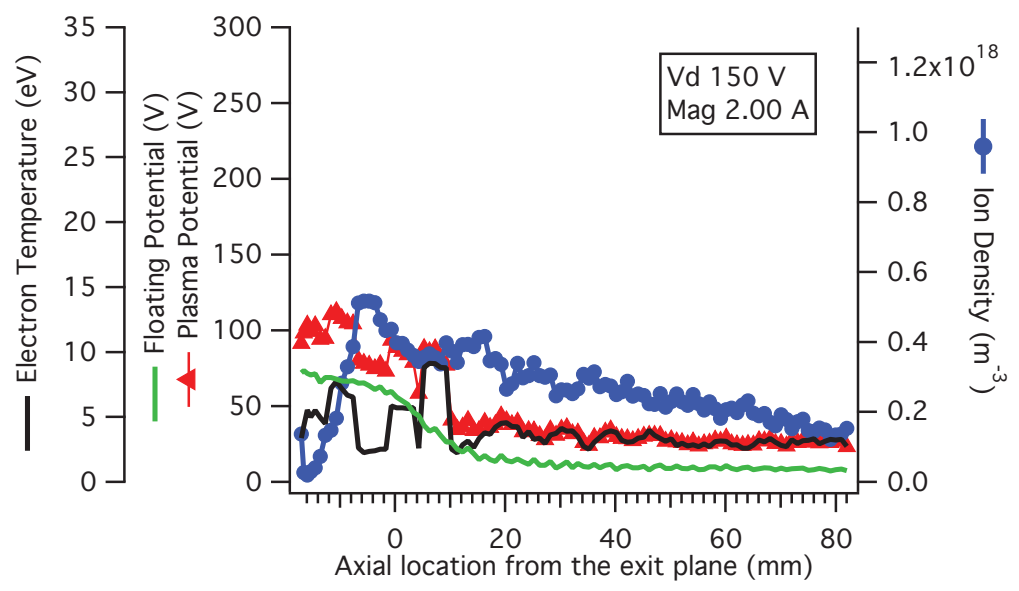

Figure A.35: High-speed probe results for Krypton

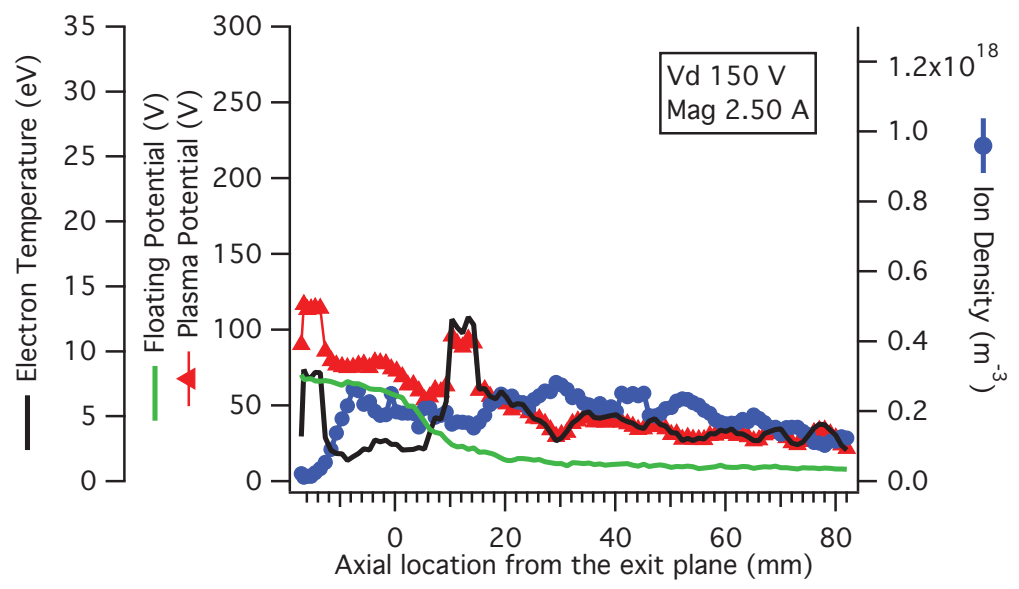

Figure A.36: High-speed probe results for Krypton 


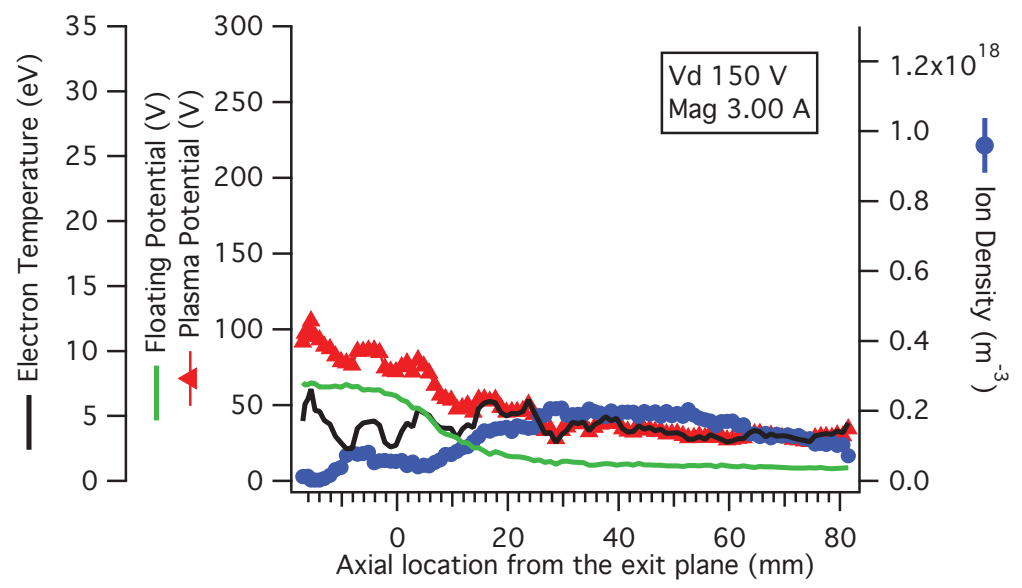

Figure A.37: High-speed probe results for Krypton

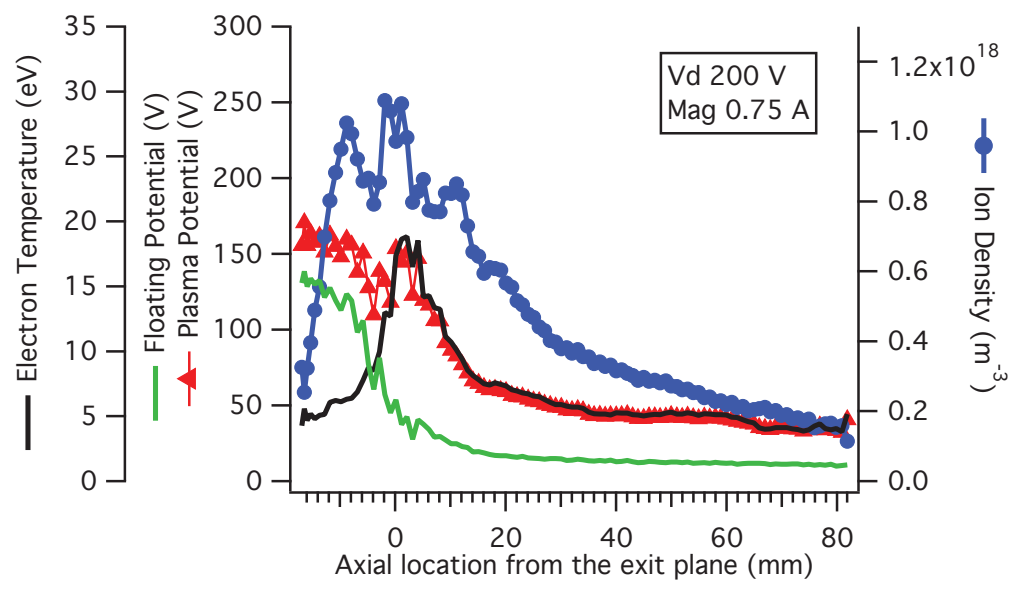

Figure A.38: High-speed probe results for Krypton 


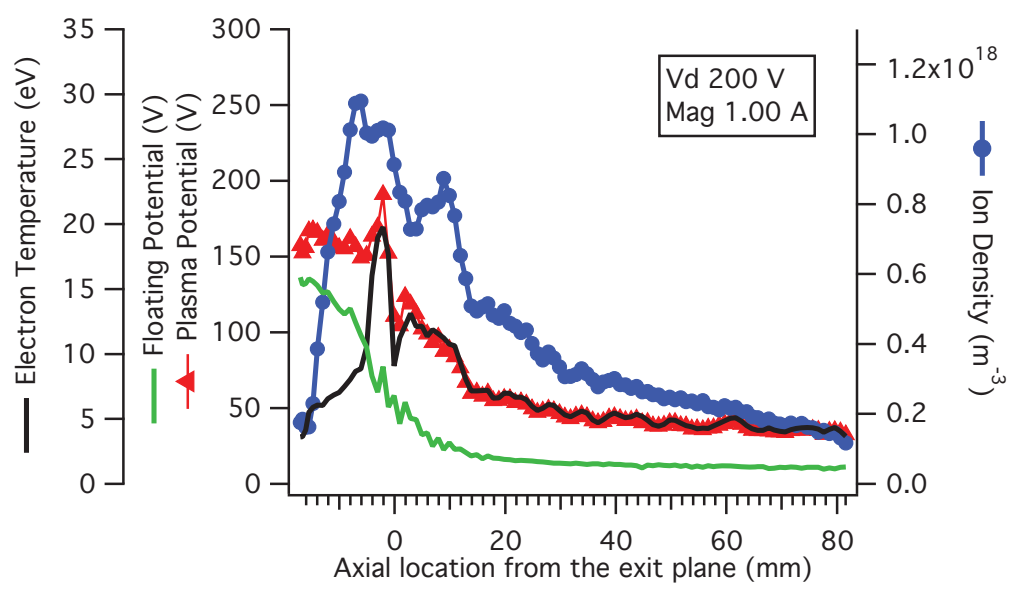

Figure A.39: High-speed probe results for Krypton

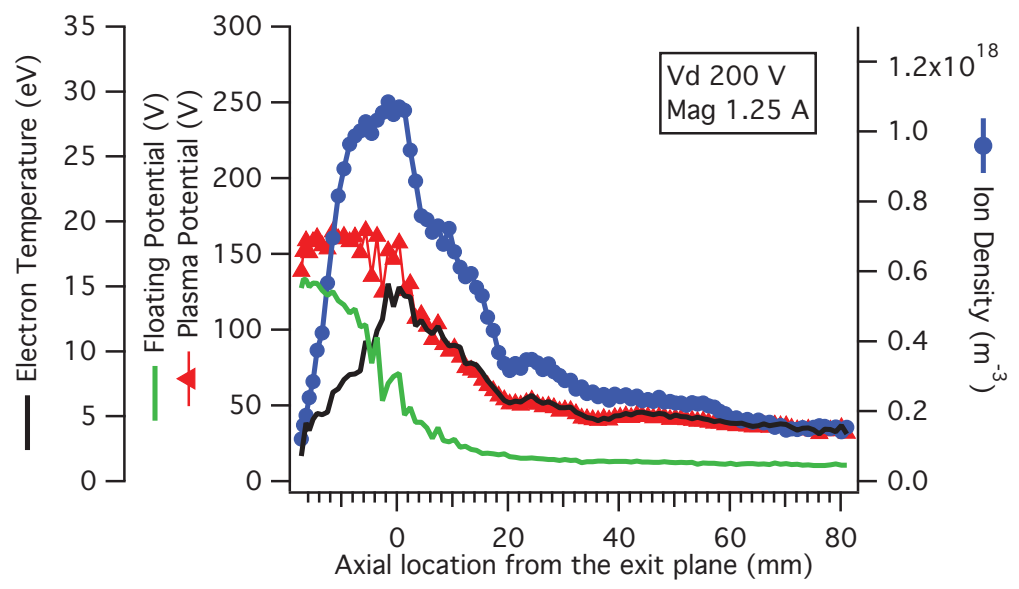

Figure A.40: High-speed probe results for Krypton 


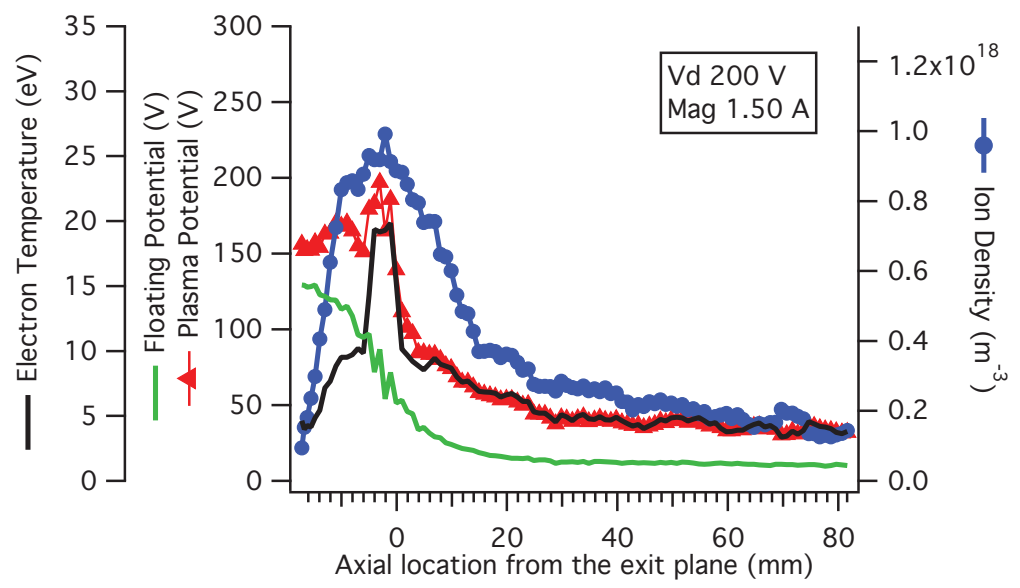

Figure A.41: High-speed probe results for Krypton

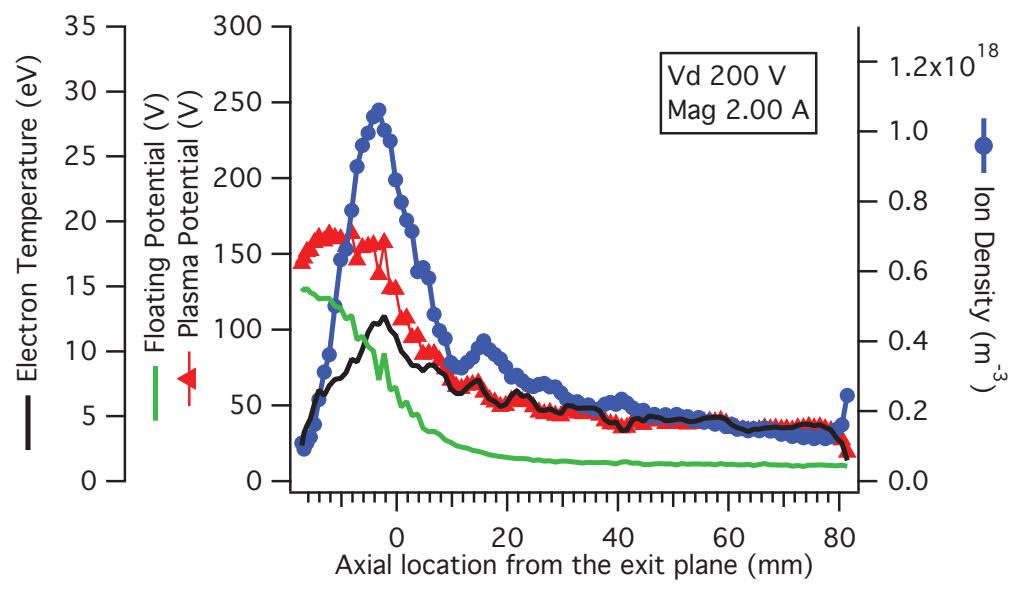

Figure A.42: High-speed probe results for Krypton 


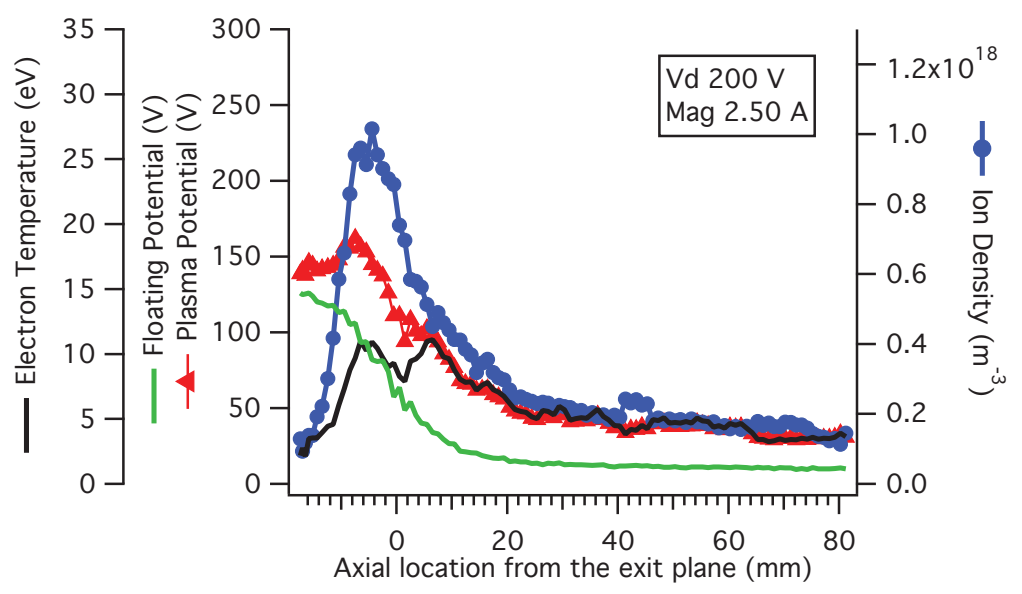

Figure A.43: High-speed probe results for Krypton

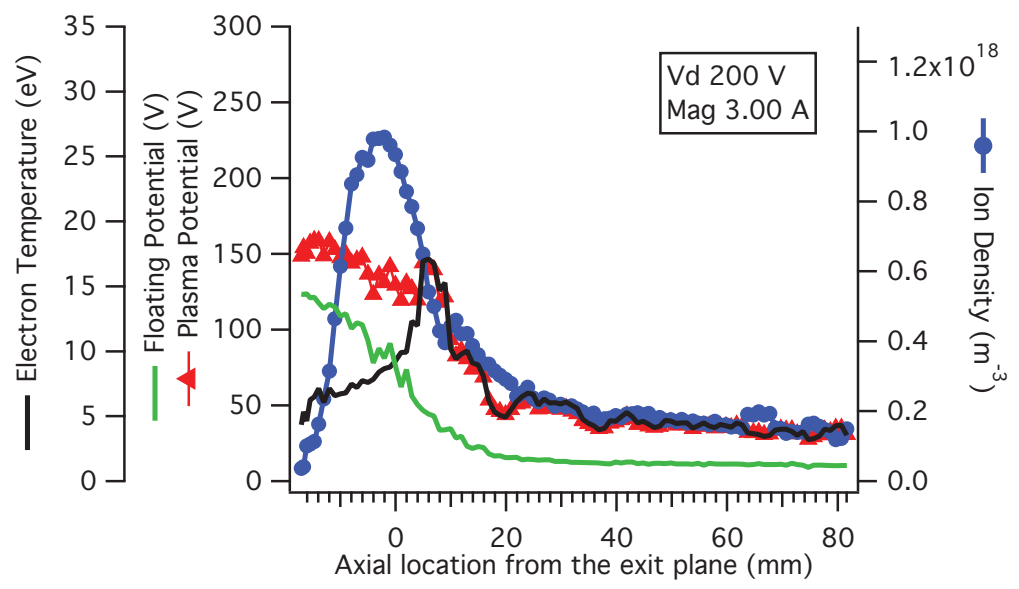

Figure A.44: High-speed probe results for Krypton 


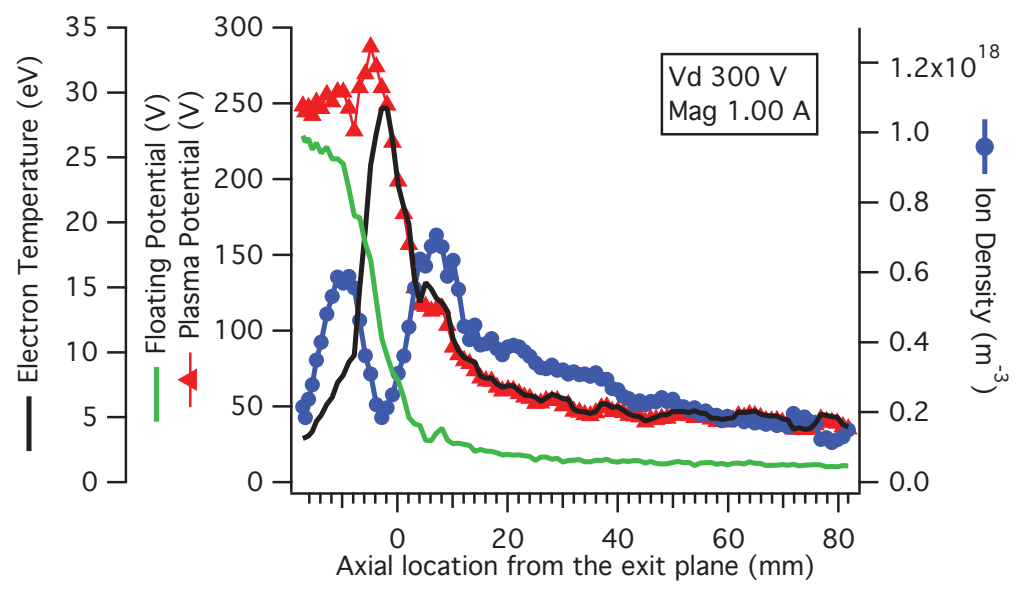

Figure A.45: High-speed probe results for Krypton

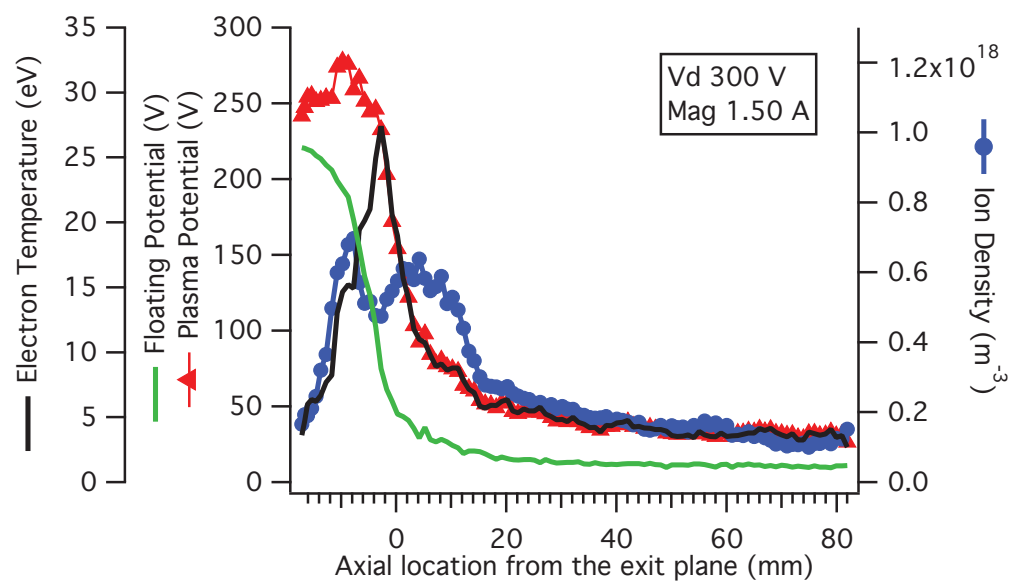

Figure A.46: High-speed probe results for Krypton 


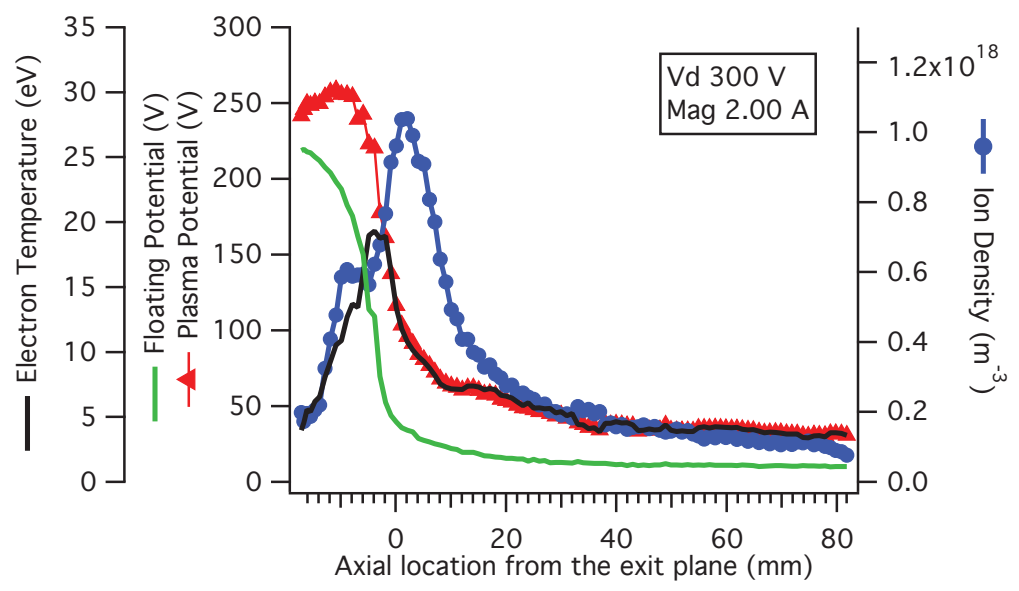

Figure A.47: High-speed probe results for Krypton

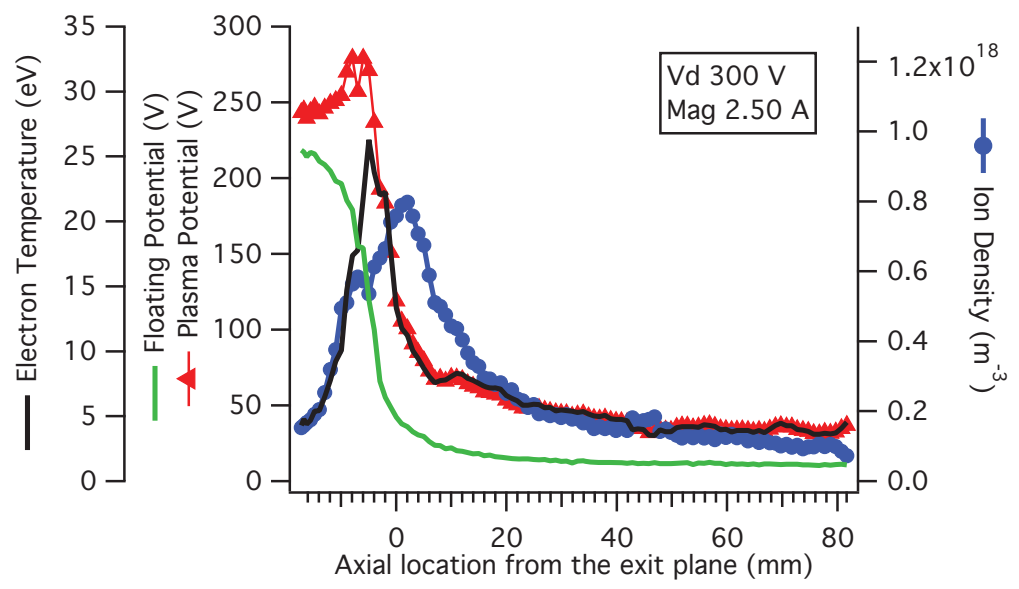

Figure A.48: High-speed probe results for Krypton 


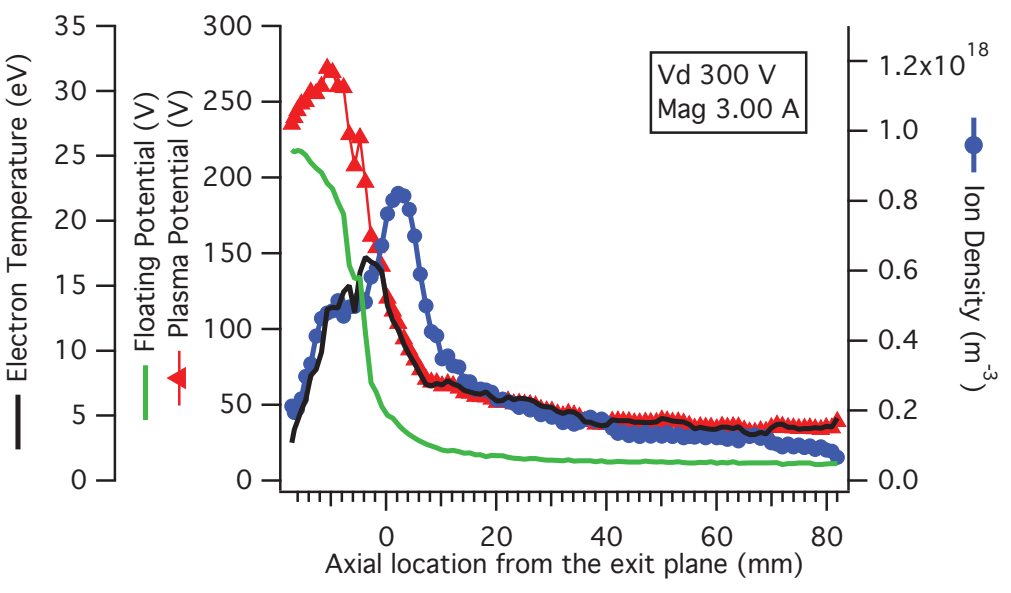

Figure A.49: High-speed probe results for Krypton 